\title{
Understanding Visual Absorption from First Principles
}

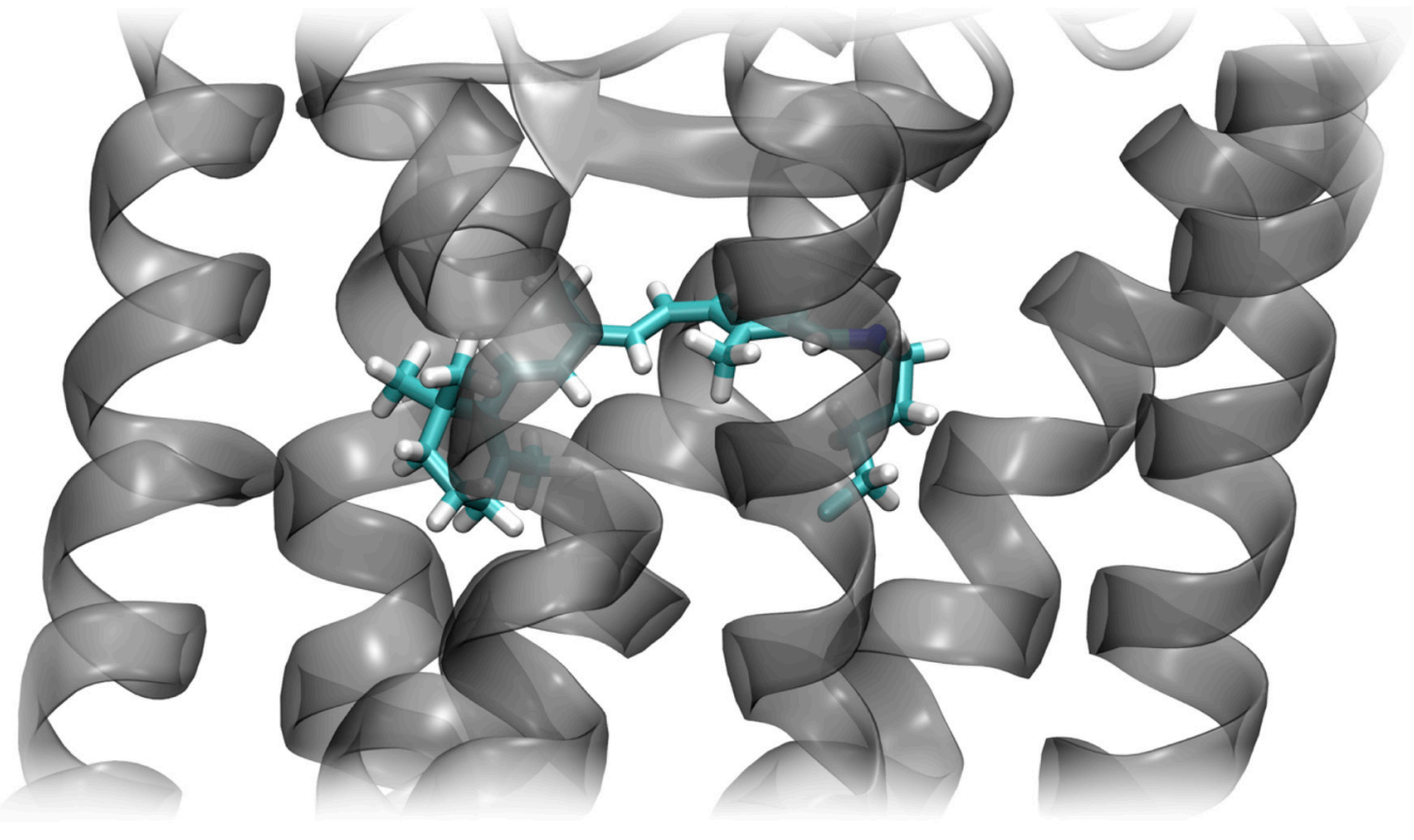

0
3
3
8
0
0
0

\section{Omar Valsson}


UNDERSTANDING VISUAL ABSORPTION FROM FIRST PRINCIPLES

Omar Valsson 
Promotion committee:

Prof. dr. G. van der Steenhoven

Prof. dr. G. van der Steenhoven

Prof. dr. C. Filippi

Prof. dr. W. J. Briels

Prof. dr. J. Herek

Prof. dr. J. Neugebauer

Dr. F. Buda

Prof. dr. G. Groenenboom
University of Twente, Chairman

University of Twente, Secretary

University of Twente, Promotor

University of Twente

University of Twente

Technical University Braunschweig

University of Leiden

Radboud University Nijmegen

O. Valsson

Understanding Visual Absorption from First Principles

Ph.D. Thesis, University of Twente, Enschede

ISBN: 978-90-365-365-3411-6

Copyright (C) 2012 by O. Valsson

No part of this work may be reproduced by print, photocopy or any other means without the permission in writing from the author.

DOI: $10.3990 / 1.9789036534116$

Online version: http://dx.doi.org/10.3990/1.9789036534116

Omar Valsson

omar.valsson@gmail.com

Printed by: Gildeprint Drukkerijen - www.gildeprint.nl 
UNDERSTANDING VISUAL ABSORPTION FROM FIRST PRINCIPLES

\section{DISSERTATION}

to obtain

the degree of doctor at the University of Twente, on the authority of the rector magnificus, Prof. dr. H. Brinksma, on account of the decision of the graduation committee, to be publicly defended on Friday $7^{\text {th }}$ of September 2012 at 14:45

by

\section{Omar Valsson}

born on March 8, 1983

in Reykjavik, Iceland 
This doctoral dissertation is approved by:

Prof. dr. C. Filippi

Promotor 


\section{Contents}

1 Introduction $\quad 1$

1.1 Vision at the Molecular Level . . . . . . . . . . . . . . . . . 1

1.2 Rhodopsin . . . . . . . . . . . . . . . . 4 4

1.3 The Spectral Tuning . . . . . . . . . . . . . . . . 6

1.4 Absorption of Rhodopsin: The Right Answer for the Wrong Reason? 8

1.5 This Thesis . . . . . . . . . . . . . . . . 10

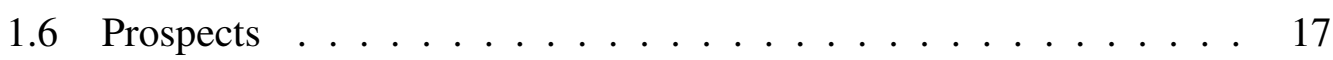

1.7 Bibliography ........................ 19

2 Theoretical Methods $\quad 23$

2.1 Introduction . . . . . . . . . . . . . . . . . 23

2.2 Quantum Mechanical Calculations . . . . . . . . . . . . . 23

2.3 QM/MM Calculations . . . . . . . . . . . . . . . . . . . . . 37

2.4 Computational Details . . . . . . . . . . . . . . 38

2.5 Bibliography .......................... 39

3 Photoisomerization of the Retinal Chromophore in a New Light 43

3.1 Introduction . . . . . . . . . . . . . . . . . 43

3.2 Methods . . . . . . . . . . . . . . . . 46

3.3 Computational Details . . . . . . . . . . . . . 50

3.4 Retinal Models . . . . . . . . . . . . . . . . . . 51

3.5 Vertical Excitation Energies . . . . . . . . . . . . . . . . 53

3.6 In-plane Geometrical Optimization . . . . . . . . . . . . . . . . . 59

3.7 Out-of-Plane Relaxation . . . . . . . . . . . . . . . . . 66

3.8 Discussion and Conclusions _ . . . . . . . . . . . . . 75

3.9 Bibliography ...................... 77

4 Excitation Energies of Retinal Chromophores: Role of the Structural Model $\quad 85$

4.1 Introduction . . . . . . . . . . . . . . . . . . 85

4.2 Computational Details . . . . . . . . . . . . . . . . . . . 87

4.3 Retinal Models . . . . . . . . . . . . . . . . . . . . . 88

4.4 Vertical Excitation Energies . . . . . . . . . . . . . . . . . . . . 89

4.5 Choice of Ground-State Geometry . . . . . . . . . . . . . . . . . . 91 
4.6 Discussion and Conclusions . . . . . . . . . . . . . 93

4.7 Bibliography .......................... 95

5 Gas-Phase Retinal Spectroscopy: Temperature Effects Are But a Mi$\begin{array}{ll}\text { rage } & 101\end{array}$

5.1 Introduction . . . . . . . . . . . . . . . . 101

5.2 Results . . . . . . . . . . . . . . . . . . . . . . . . . . . . . . . 104

5.3 Discussion and Conclusion . . . . . . . . . . . . . . 109

5.4 Bibliography ...................... 110

6 Native Description of Visual Absorption 113

6.1 Introduction . . . . . . . . . . . . . . . . . 113

6.2 Methods . . . . . . . . . . . . . . . . . . . 116

6.3 Results . . . . . . . . . . . . . . . . . . . . . 119

6.4 Discussion and Conclusions . . . . . . . . . . . . . 133

6.5 Bibliography ....................... 136

7 Electronic Excitations of Simple Cyanine Dyes 143

7.1 Introduction . . . . . . . . . . . . . . . . . . . . . . . 144

7.2 Computational Details . . . . . . . . . . . . . . . . . . 145

7.3 Vertical Excitation Energies . . . . . . . . . . . . . 147

7.4 Discussion . . . . . . . . . . . . . . . . . 161

7.5 Conclusion . . . . . . . . . . . . . . 167

7.6 Bibliography . . . . . . . . . . . . . . 168

$\begin{array}{ll}\text { List of publications } & 173\end{array}$

$\begin{array}{lr}\text { Curriculum vitae } & 175\end{array}$

$\begin{array}{lr}\text { Acknowledgements } & 179\end{array}$ 


\section{Chapter 1}

\section{Introduction}

\subsection{Vision at the Molecular Level}

Vision is one of the most fundamental processes in biology and has fascinated scientists for centuries. In the 19th century, Thomas Young and Hermann von Helmholtz developed their theory of trichromatic color vision, namely, that primate color vision is due to the existence of three types of photoreceptors in the retina, which are sensitive to red, green, and blue light. The pioneer in the scientific study of the visual process at the molecular level was George Wald who, in the 1930's, discovered the function of vitamin $\mathrm{A}$ in vision and why its deficiency causes night blindness. He later made considerable advances in unraveling the chemical process of vision, for which he was awarded The Nobel Prize in Physiology or Medicine in 1967 [1]. Still today, the visual process is the subject of intensive investigation since the mechanism at the molecular level is not fully understood [2-30].

Vision is highly adaptive to light intensity and operates over a range of almost 8 orders of magnitude. This is achieved by having two types of photoreceptor cell in the retina, rods and cones, responsible for dim light and color vision, respectively. In the retina, there around 130 million photoreceptor cells, with the rods outnumbering the cones by a factor of around 20. There is only one type of rod cells while, in trichromats like humans, there are three types of cone cells, red, green, and blue, which facilitate color discrimination. The rods are able to detect a single photon while the cones are a factor of 100 less sensitive. However, the rods saturate at a low intensity of around 1000 photons per second while the cones are able to operate up to an intensity of 100 million photons per second. Therefore, most of the time, primates make use of the cones for visual perception, which enable color vision, while the rods take over in dim light, resulting in loss of color vision.

At the molecular level, the process of vision is regulated by the visual opsins pigments, which are the transmembrane proteins located in the rods and cones. These proteins absorb light and initiate the visual transduction process. Rhodopsin is the visual opsin in the rods while, in the cones, we have the red, green, and blue-cone opsins. Specifically, the opsins are located in membrane disks, which are flat vesicles located in the outer segments of the rod and cone cells. As depicted in Figure 1.1, 
we can think of the rod outer segment as a cylindrical container, between 30 to 60 $\mu \mathrm{m}$ in length and 1.4 to $10 \mu \mathrm{m}$ in diameter, stacked with about 1000 membrane disk, each containing of the order of 100 thousand embedded Rhodopsin proteins. Therefore, with around 130 millon rod cells in the eye, each with about 100 million Rhodopsins, the total number of Rhodopsins in the eye is on the order of $10^{16}$.

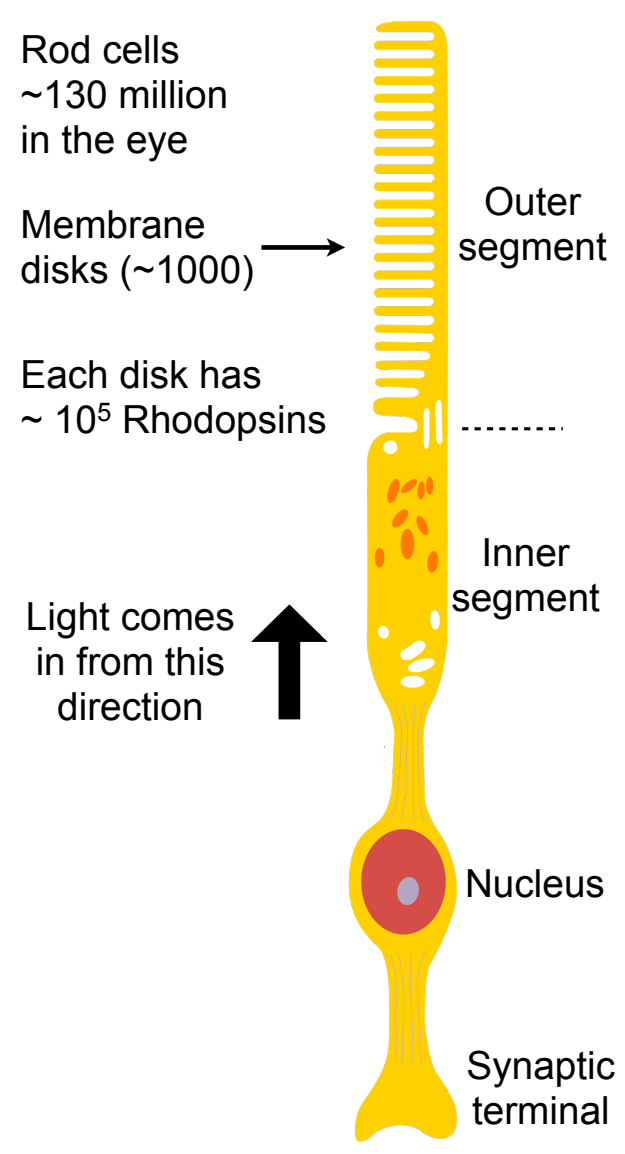

Figure 1.1: The rod photoreceptor cell (adapted from Ref. 31).

All the opsins have the same global structure of seven $\alpha$-helices that span the membrane while their amino acid sequences differ. Remarkably, most visual opsins contain the same photosensitive molecule, the 11-cis retinal chromophore, and it is only the difference in protein environment that tunes their absorption. For example, in the cone opsins, the absorption maximum is tuned from the red $(560 \mathrm{~nm} / 2.21$ $\mathrm{eV})$ to the blue $(420 \mathrm{~nm} / 2.95 \mathrm{eV})$ over an impressive range of almost $140 \mathrm{~nm}(0.75$ $\mathrm{eV})$ while Rhodopsin falls in the middle with an absorption maximum at about 498 $\mathrm{nm}(2.49 \mathrm{eV})$.

Another fascinating aspect of the opsins is the primary event in vision, that is, the photoisomerization of the retinal chromophore, which initiates the visual transduction process. Upon absorption of light, the retinal chromophore undergoes excitedstate cis-trans isomerization of one of its double bond, which results in the all-trans 
retinal chromophore. In Rhodopsin, this is ultrafast and completed within 200 fs as well as highly efficient with a high quantum yield of $67 \%$ for the final photoproduct. The local geometrical change of the chromophore inside the constrained protein pocket induces then large-scale changes in the overall structure of the protein. These structural changes result in a series of distinct intermediate with different absorption maxima and lead to the active state of Rhodopsin, meta II, which activates further signaling pathways inside the membrane. Finally, the protein and the retinal chromophore dissociate and, only after a series of chemical reactions, the Rhodopsin protein is regenerated. It is here where the connection between vitamin A deficiency and night blindness enters into the story: The retinal chromophore is derived from vitamin A, and insufficient supply of vitamin A inhibits the regeneration of Rhodopsin, leading to night blindness.

Over several decades, significant research effort has been devoted to further our understanding of the molecular mechanism underlying the complex functioning of the visual opsins as it testified by the numerous experimental [2-30] and theoretical [32-45] studies which have mainly focused on Rhodopsin. In particular, bovine Rhodopsin has been characterized very well experimentally, mainly because it can be easily extracted from cattle eyes, of which there is almost an endless supply from slaughterhouses due to human craving of beef. For example, for bovine Rhodopsin, there are numerous crystallographic structures available [27-30] while, for the other visual opsins, there are almost no crystallographic structures available. For the human cone opsins, there are homological models based on Rhodopsin but, given the low sequence identity between the cone opsins and Rhodopsin, they might not be sufficiently accurate.

In this thesis, we investigate Rhodopsin from a theoretical point of view and focus on the description of its absorption properties from first principles. To achieve this goal, due to complexity of the system, we have to employ a multiscale approach and a hierarchy of theoretical techniques to bridge between the smaller scale of the photosensitive chromophore and the much larger environment given by the protein embedded in its membrane, as we describe in Chapter 2.

One may ask why we focus on absorption. First, understanding which interactions of the chromophore-protein complex affect the Rhodopsin absorption spectrum will greatly help to elucidate the more general aspect of spectral tuning in visual opsins. Second, Rhodopsin can be considered the exemplar photosensitive biosystem as it displays all possible complications which render its theoretical description extremely challenging. As it is explained below, the electronic charge in the retinal chromophore responds rather strongly to photo-excitation and is significantly affected by the surrounding environment. Moreover, a realistic description of the structure of the chromophore in the protein pocket is essential to capture the response of the system to light. Therefore, if our theoretical tools are able to provide an accurate description of the absorption properties of Rhodopsin, we will be in a position to tackle almost any photosensitive system.

However, after a quick inspection of the abundant theoretical literature on the subject, another legitimate question the reader may have is why we should revisit 
the theoretical description of the absorption of Rhodopsin at all. This problem has in fact already been considered in numerous theoretical studies, which have often claimed remarkable agreement with experiments. Therefore, is this problem already solved? Should we not move on to other opsins? As we discuss in detail later in this Chapter, a closer look at previous theoretical studies on Rhodopsin reveals that things are not so clear, and that the problem is not at all solved.

In the following, we will begin by describing the structure of Rhodopsin and of the retinal chromophore. We will also explain the nature of the excitation of the chromophore and how the interaction between the protein and the chromophore can tune its absorption. We will then briefly summarize previous results and, finally, describe the results obtained in this thesis.

\subsection{Rhodopsin}

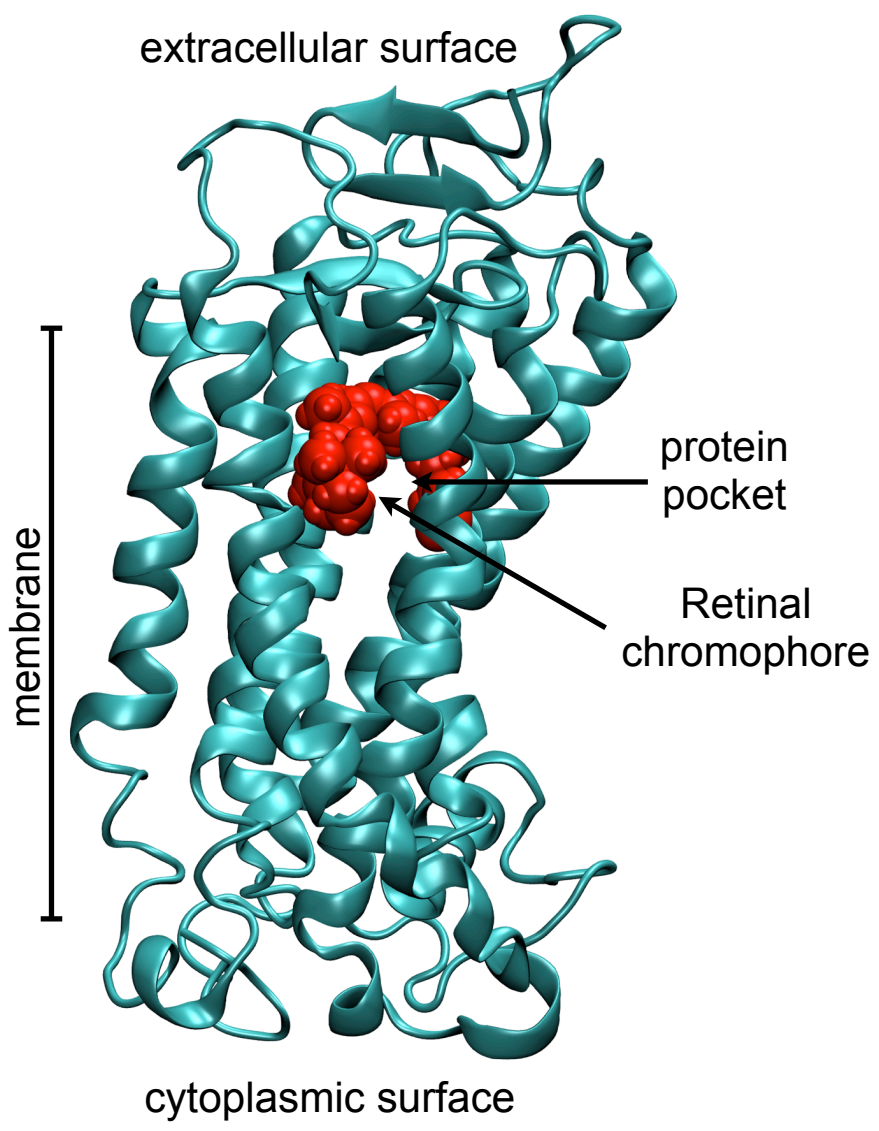

Figure 1.2: The global structure of Rhodopsin consists of seven $\alpha$-helices that span the membrane. We indicate the approximate range of the membrane and show the retinal chromophore inside the protein pocket. 
Rhodopsin [22-26] consists of 348 amino acids residues, of which $65 \%$ are located in the transmembrane region. As shown in Figure 1.2, the global structure is mainly given by seven $\alpha$-helices that span the membrane and vary in length from 20 to 33 residues. The helices are connected by three loops, both on the cytoplasmic (inside the membrane) and the extracellular (outside the membrane) surfaces. Furthermore, on the cytoplasmic surface, there is a short $\alpha$-helix, that lies parallel to the membrane. The length of Rhodopsin is about $70 \AA$ along the axis perpendicular to the membrane while the diameter is about 30 to $40 \AA$.

The photosensitive 11-cis retinal chromophore is located inside a protein pocket that is buried between the helices, close to the extracellular surface, and covered by one of the loops on the extracellular side. The environment of the chromophore in the protein pocket is largely hydrophobic, although there are some polar residues nearby.

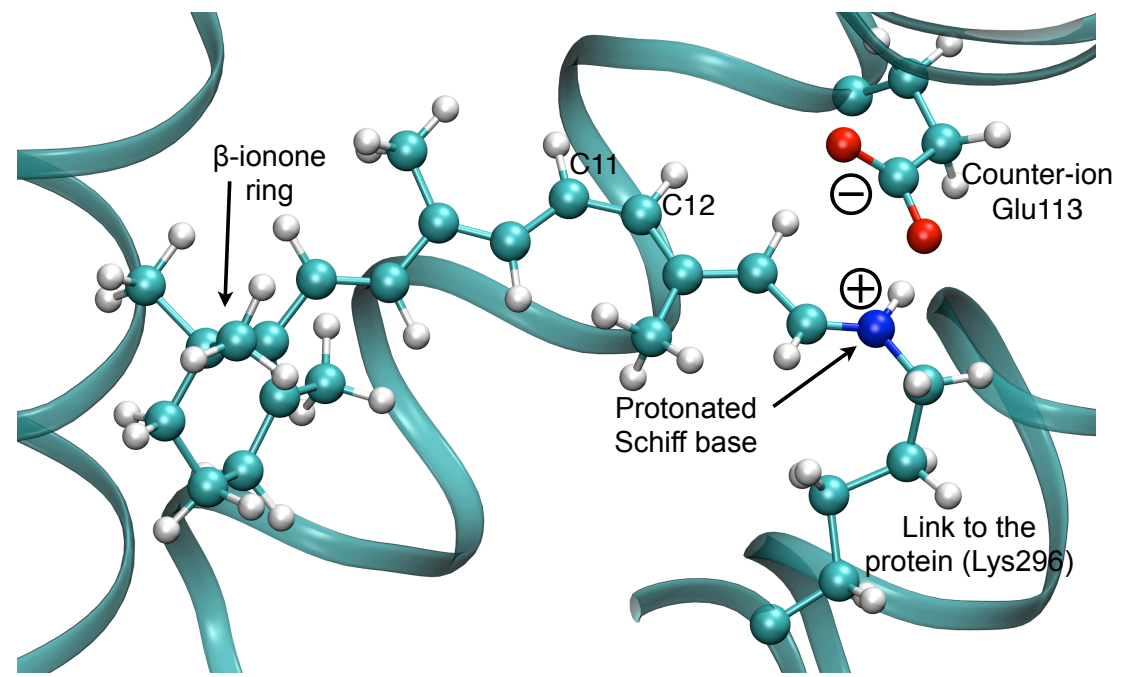

Figure 1.3: The retinal chromophore and the glutamic acid counter-ion (Glu113) inside the protein pocket in Rhodopsin.

As shown in Figures 1.3 and 1.4a, the retinal chromophore consists of a conjugated chain, which extends from the N16 nitrogen towards the C5 carbon, and a $\beta$-ionone ring. In the protein, the retinal chromophore is covalently linked to a lysine residue (Lys296) via a protonated Schiff base linkage. All the double bonds along the conjugated chain are in a trans configuration, except the $\mathrm{C} 11-\mathrm{C} 12$ bond that is in a cis configuration.

Due to the protonated Schiff base, the chromophore is positively charged with most of the charge localized around the nitrogen. Therefore, a negatively charged glutamic acid counter-ion (Glu113) is located in close proximity of the protonated Schiff base, at around $3 \AA$ distance from the nitrogen. This counter-ion stabilizes the proton on the chromophore by increasing the $\mathrm{p} K_{\mathrm{a}}$ of the Schiff base to more than 15 . Furthermore, as we discuss in more detail below, the counter-ion has an important role in the spectral tuning, blue-shifting the absorbance of the isolated chromophore. 


\subsection{The Spectral Tuning}

All visual opsins have the same global protein structure of seven transmembrane $\alpha$ helices, and most of them also incorporate the same chromophore, that is, the retinal chromophore. Differences in the amino acid sequence and the resulting changes in the interaction between the chromophore and the protein modulate the absorption maximum over a wide range in the visible spectrum, from 420 to $590 \mathrm{~nm}$. For example, let us consider the human red, green, and blue-cone opsins which have absorption maxima of $560 \mathrm{~nm}, 530 \mathrm{~nm}$, and $420 \mathrm{~nm}$, respectively. The red and green cone opsins are rather similar and differ only in 15 amino acids, which results in a $30 \mathrm{~nm}$ difference in the absorption maxima. On the other hand, the red and blue cone opsins have only a sequence identity of $43 \%$ and, perhaps not surprisingly, are characterized by a $140 \mathrm{~nm}$ difference between them. Then, compared to bovine Rhodopsin, the red, green, and blue opsin have amino acid sequence identity of $37 \%$, $38 \%$, and $41 \%$, respectively.

Before we discuss how the interaction with the protein environment tunes the absorption of the retinal chromophore, we need to understand the nature of the excitation of the isolated chromophore. As previously mentioned, the retinal chromophore is positivity charged with most of the charge localized around the nitrogen on the protonated Schiff base when the system is in the ground state. As shown in Figure 1.4, in a simple orbital picture, the highest occupied molecular orbital (HOMO) is a bonding $\pi$ orbital delocalized on the conjugated chain of the chromophore while the lowest unoccupied molecular orbitals (LUMO) is an anti-bonding $\pi^{*}$ orbital. The bright excited state is mainly a HOMO to LUMO transition or a $\pi$ to $\pi^{*}$ excitation, and results in a transfer of positive charge along the chain of the chromophore, from the protonated Schiff base towards the $\beta$-ionone ring. This is clearly seen in Figure 1.4, where the difference between the excited- and the ground-state density is shown.

When going from the gas phase to the protein, a factor which affects the absorption properties is the geometrical distorsion. In the gas phase, the isolated chromophore is characterized by a planar conjugated chain while, inside the protein pocket, steric interactions distort the chromophore from planarity. This leads to a shorter effective length of the conjugated chain and, as expected from a simple particle-in-a-box reasoning, a blue shift in absorption. Depending on the presence of either less or more bulky amino acids in the protein pocket, the steric interaction will differ between opsins and, consequently, the degree of distortion of the chromophore will also be different. The resulting shorter or longer effective length of the chain can therefore modulate the absorption between different opsins. Furthermore, the conjugated chain of the chromophore extends into the $\beta$-ionone ring, so the absorption will also be affected by the twisting of the ring, which is also determined by steric effects in the protein pocket.

A more important factor of the spectral tuning is the polarization of the chromophore resulting from the electrostatic interaction with the protein. Due to the large difference between the charge distribution of the ground and excited states 


\section{a}

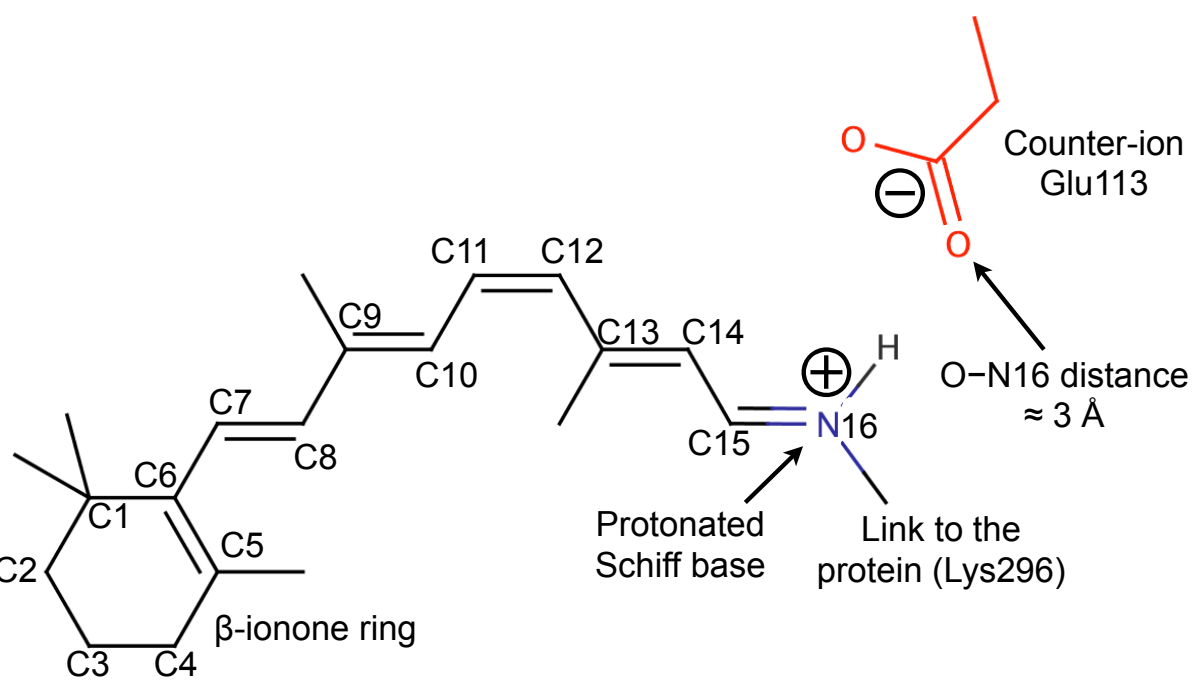

b

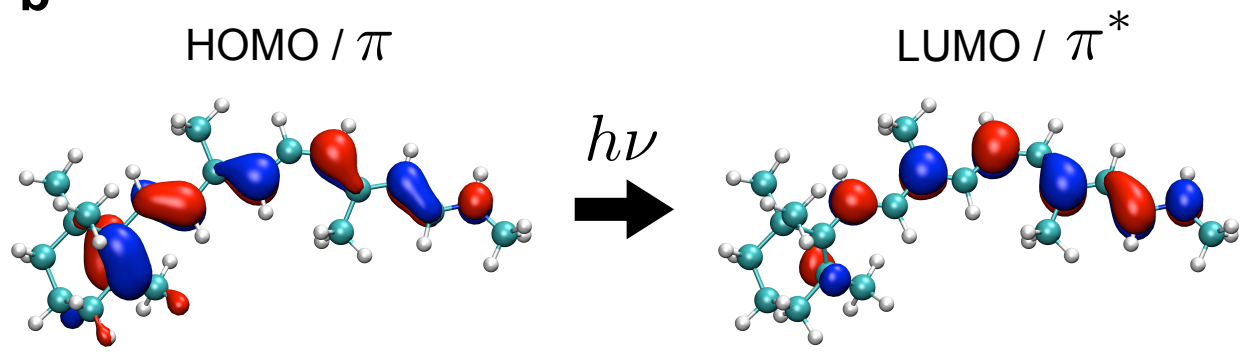

C

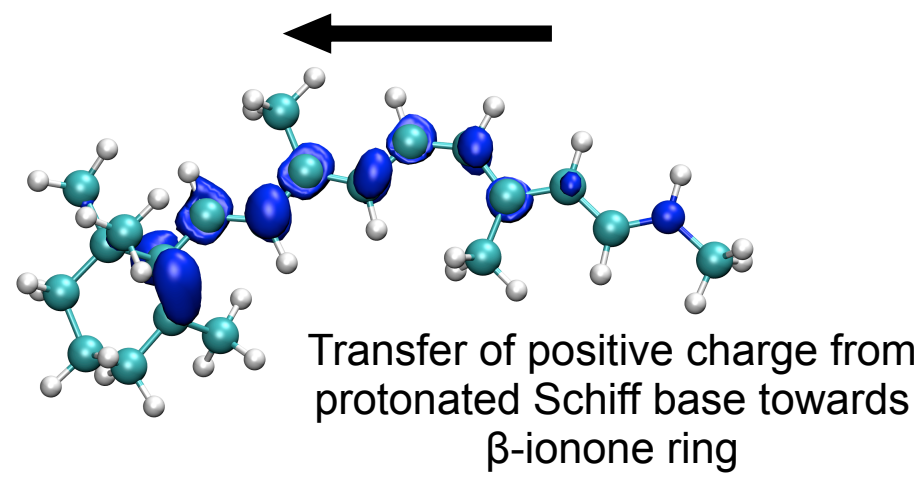

Figure 1.4: a) The retinal chromophore; b) the highest occupied (HOMO) and lowest unoccupied (LUMO) molecular orbitals; c) difference between the excited- and ground-state densities. 
of the chromophore, this effect can be significant. This can be most easily understood by considering the effect of the counter-ion on the excitation energy of the system. As discussed above, the chromophore is positively charged with most of the charge localized around the protonated Schiff base in the ground state, and near the $\beta$-ionone ring in the excited state as a result of photo-induced charge transfer. The presence of a negative charge near the protonated Schiff base, like the one provided by the counter-ion, will stabilize the ground state more than the excited state. This will tend to open the gap and will lead to a blue shift as compared to the isolated chromophore. Consequently, the distance between the protonated Schiff base and the counter-ion, which might differ between opsins, can strongly modulate absorption as shorter distances will cause a more significant blue shift. On the other hand, we expect that introducing a negative charge near the $\beta$-ionone ring will stabilize the excited state more than the ground state, and therefore close the gap leading to a red shift.

The rest of the protein environment will also affect the polarization of the chromophore. Charged, polar, or polarizable amino acid residues in the protein pocket will interact electrostatically with the chromophore and either stabilize more the ground or the excited state, contributing to either a blue or a red shift, respectively. Due to the variations in amino acid sequences between different opsins, the overall electrostatic interaction between the protein and chromophore will be different, and affect the absorption of the chromophore.

Moreover, we note that variations in the absorption maxima of some visual opsins can be explained as due to differences in the photosensitive chromophore itself. For example, it is thought that opsins absorbing in the ultra-violet range, like in mouse eyes, have the retinal chromophore in a deprotonated (neutral) form, which leads to an absorption maximum blue-shifted at approximately $360 \mathrm{~nm}$. Furthermore, visual opsins in some species are known to incorporate an 11-cis-3,4dehydroretinal chromophore with a longer conjugated chain due to an extra double bond in the $\beta$-ionone ring, which shifts the absorption to the red, resulting for example in a maximum as high as $630 \mathrm{~nm}$.

Spectral tuning is therefore the complex combination of different effects, both geometrical and electrostatic. Often, these factors counteract each other and it is hard to discern between the different contributions to the final absorbance. In principle, accurate theoretical calculations would be ideal to understand what affects the photophysics of the chromophore, as they allow us to "play" with the system, for instance by turning off or modifying specific amino acids in the surrounding of the chromophore.

\subsection{Theoretical Absorption of Rhodopsin: The Right Answer for the Wrong Reason?}

A breakthrough in Rhodopsin research came in the years 2000 to 2004 when highquality crystallographic structures became available [27-30]. This prompted a mul- 
titude of theoretical investigations aimed at describing the absorption of Rhodopsin from first principle calculations [32-40]. Most of these studies report rather impressive results with excitation energies that deviate by less than $0.1 \mathrm{eV}$ from the experimental absorption maximum at $2.49 \mathrm{eV}$. This good performance is quite remarkable given that these studies often differ considerably in the theoretical approaches employed and normally resort to various approximations in the treatment of the chromophore-protein system. In view of this apparent success, one is lead to conclude that absorption in Rhodopsin is a solved problem and that achieving an accurate theoretical description of the photophysics of a complex photobiological system is routinely possible with nowadays computational techniques. In fact, this thinking has prompted many authors of these studies to believe that they had calibrated their computational tools and could turn their attention to understanding the absorption tuning between the different visual opsins, like the color opsins [42-45]

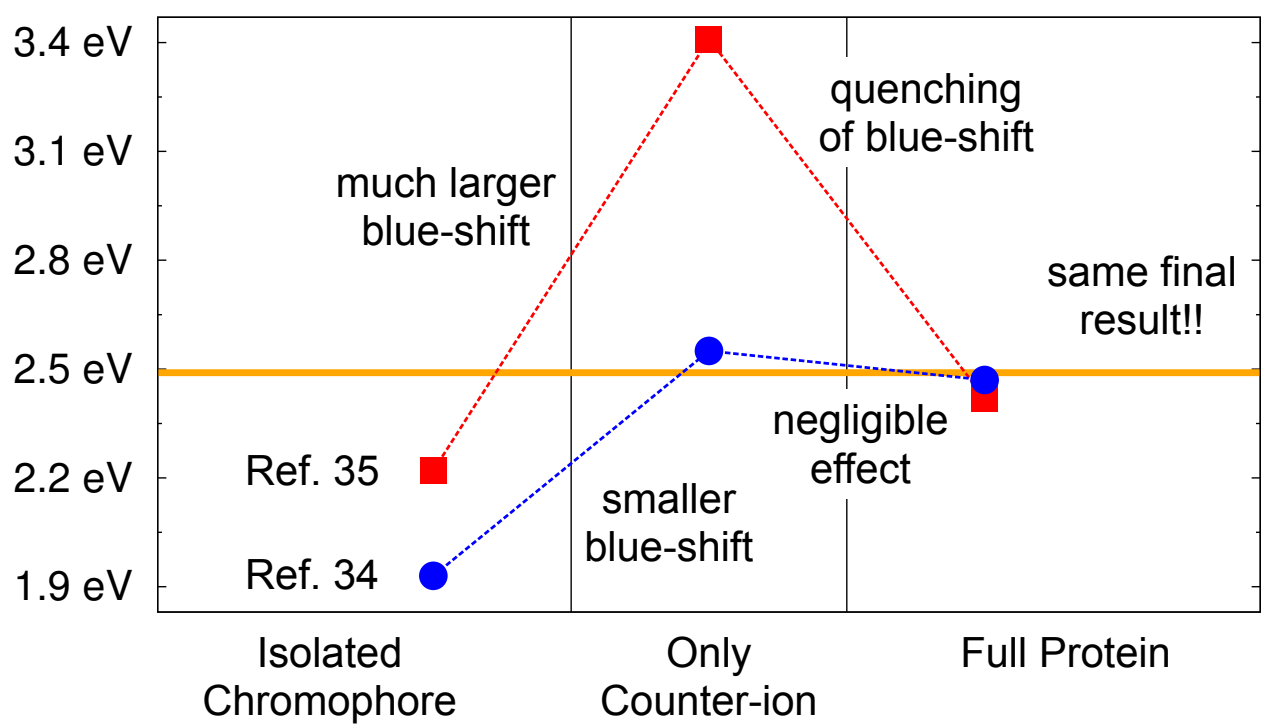

Electrostatic Environment

Figure 1.5: Absorption of Rhodopsin from Refs. 34 and 35. The excitation energies are obtained with the structure of the chromophore relaxed in the protein, and with different contributions of the electrostatic environment.

However, if we analyse more carefully the theoretical literature on Rhodopsin, we quickly realize that the picture emerging is rather murky. For example, let us consider the two prototypical studies of Refs. 34 and 35, which obtain excitation energies of $2.47 \mathrm{eV}$ and $2.42 \mathrm{eV}$, respectively, in rather good agreement with the experimental absorption maximum. To an eye untrained to the subtleties of theoretical chemistry, these studies might seem rather similar as they start from the same crystallographic structure and employ the same highly-correlated method to compute the excitations. However, as shown in Figure 1.5, they reach very different conclusions 
concerning the electrostatic effect of the protein environment on the excitation energy of the chromophore. In Ref. 34, the main effect of the protein is due to the counter-ion, which induces a significant blue shift, while the rest of the protein environment has a negligible effect. On the other hand, Ref. 35 obtains a much larger blue shift due to the counter-ion, which is then quenched by the rest of the protein environment. Without going here into detail, we note that the critical difference between these two studies are the methods employed to obtain the ground-state geometry of chromophore (the construction of a realistic structural model of Rhodopsin will be a major focal point of this thesis).

The two investigations of Refs. 34 and 35 are just two examples but, if we were to consider other studies, the picture would become even fuzzier and the faith of the reader in first-principle calculations might be somewhat undermined. Since these contradicting calculations cannot all be right, it is clear that the apparent agreement with experiments is in most cases due to a favorable cancelation of errors. In short, they obtain the right number for the wrong reasons. Therefore, it is also clear that achieving an accurate theoretical description of the absorption of Rhodopsin is not at all a solved problem. Ultimately, this observation leads us to the question: Which theoretical ingredients are needed to achieve an accurate description? In this thesis, we will revisit the absorption of Rhodopsin and try to provide an answer to this question.

\subsection{This Thesis}

The work done in this thesis is rather broad and it may not be obvious to the reader how the individual pieces fit together. In this section, we will therefore summarize the content of the thesis, focusing on the more general picture.

To better understand this thesis, we need to explain which factors mostly influence the theoretical description of absorption in a photosensitive chromophore like retinal. There are essentially two main aspects we need to pay particular attention to: The choice of ground-state geometry and the method employed to compute the excitation energies. For the ground-state geometries, a reasonable option is to use density functional theory as it offers a good balance of accuracy and efficiency. This is also our choice but has not been the most common choice in the retinal studies of the last two decades. For the excitation energies, the choice of method is even less clear since there is a multitude of approaches available, which often lead to very different results even if we restrict ourselves to methods that are supposedly equally sophisticated. I will not go here into details but only say that we have identified a group of highly-correlated methods we believe are sufficiently accurate. Then, when considering the protein environment, we have the additional complication of how to describe the electrostatic interaction between the chromophore and the protein. As it is commonly done in the study of photobiological systems, we employ a hybrid quantum mechanical in classical mechanics (QM/MM) scheme, where the chromophore is treated with a quantum method and the protein environment with a 
classical description through the use of non-polarizable force fields. This results in the approximation that the protein environment can polarize the chromophore but cannot respond to the excitation of the chromophore.

Let us now focus on the thesis. Since previous studies give rather contradictory pictures concerning the role of the protein environment in tuning the absorption in Rhodopsin, we decided to start with something simpler, namely, the intrinsic absorption of the retinal chromophore in the gas phase, and only subsequently tackle the complete chromophore-protein system. Removing the complications of the protein allows us to thoroughly calibrate the theoretical methods employed to compute the geometrical model and the excitation energies. These studies are the focus of the first three Chapters and, as we will see, are an important part of the thesis since the description of absorption in the gas phase turned out to be not that simple.

In Chapter 3, we construct gas-phase retinal models ranging from a minimal model to the full 11-cis chromophore to investigate the performance of a wide range of theoretical approaches in the description of the vertical excitation energies. This extensive comparison allows us to identify a group of highly-correlated approaches (in particular, multi-reference perturbation and quantum Monte Carlo methods), which provide a balanced description of dynamical and static correlation, and a consistently accurate prediction of the excitation energies of retinal. These findings are further confirmed in Chapter 4, where we revisit these retinal models with a more recent flavor of many-body perturbation theory which includes twobody interactions in the zero-order Hamiltonian. Importantly, through a calibration of the methods employed to construct the ground-state structures, we also show that the theoretical procedure commonly employed in the retinal investigations of the last decade is in disagreement with more accurate approaches. Most gas-phase retinal and Rhodopsin studies (as the one from Ref. 35 in Figure 1.5) use a low-correlation technique, inferior to density functional theory, to compute the structure (i.e. the complete-active-space self-consistent space method), in combination with an superseded perturbative approach to compute the excitation energies. We demonstrate that both aspects of these previous calculations are totally inadequate to describe retinal in the gas phase. Clearly, our findings cast severe doubts about the ability of this commonly used procedure to describe absorption in the protein.

The results obtained in Chapters 3 and $\mathbf{4}$ allow us to estimate with a good degree of confidence that the vertical excitation energy of the 11-cis retinal chromophore in the gas phase is around $2.3 \mathrm{eV}$ and, therefore, in disagreement with the experimental estimate of $2.0 \mathrm{eV}$ obtained in photoinduced dissociation spectroscopy [46]. As shown in Figure 1.6a, the photo-dissociation spectrum has a rather complex structure with a main peak around $2.0 \mathrm{eV}$ and a shoulder which extents to higher energies. Could it be that the main peak at lower energies corresponds to the adiabatic excitation energy and the vertical excitation lies somewhere in the shoulder? This is of course a possibility especially for a photo-reactive molecule as retinal but, before launching into speculations, we need to describe some additional oddities regarding the comparison between theory and photo-dissociation spectroscopy experiments on the retinal chromophore. 
a) 11-cis Ref. 46

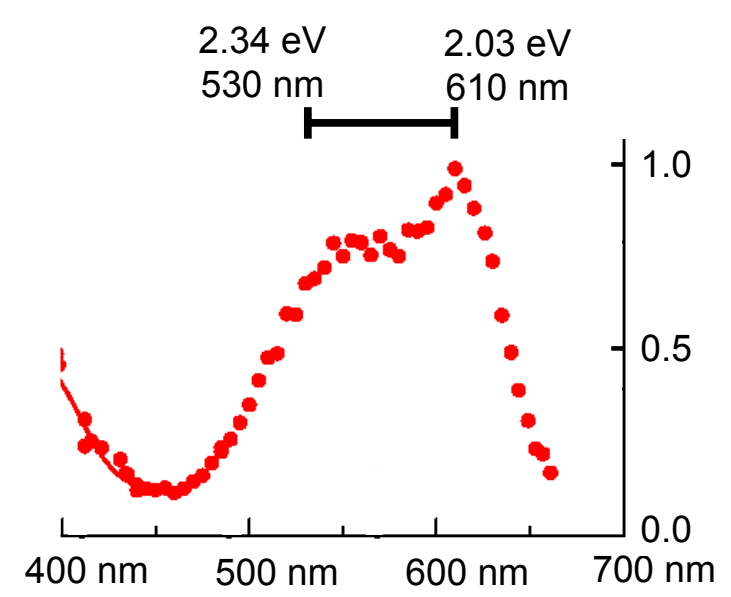

b) all-trans

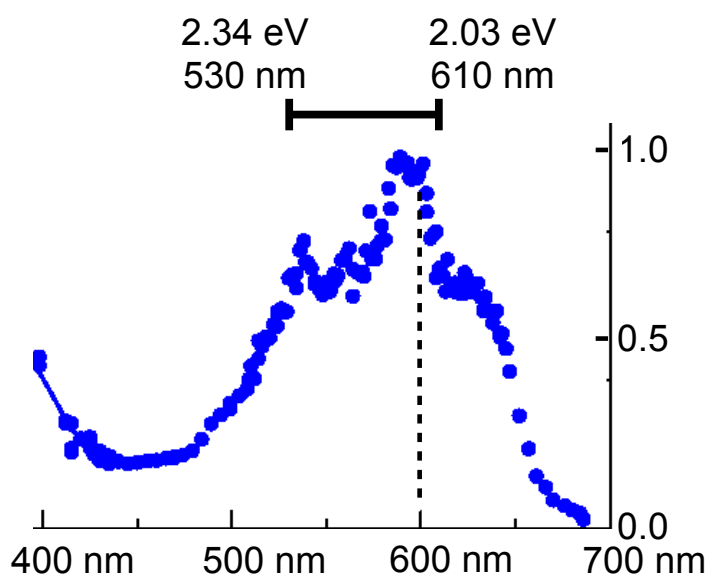

c) all-trans

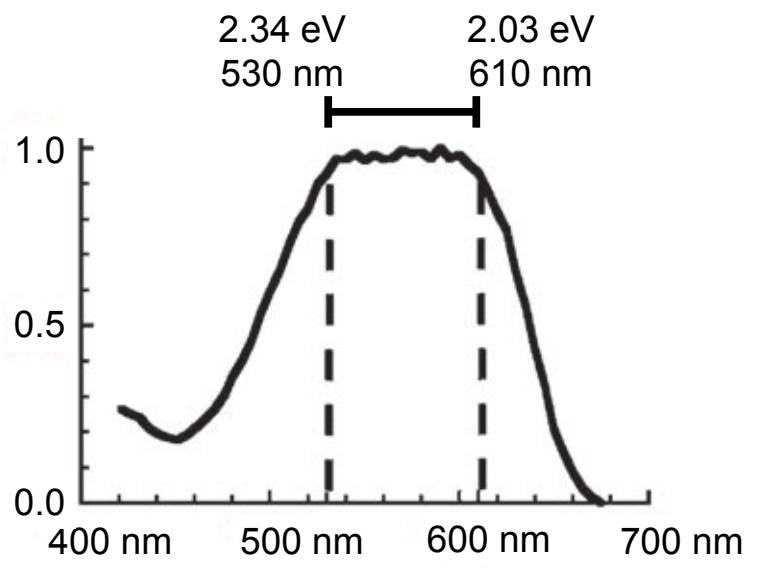

Figure 1.6: Gas-phase photodissociation spectroscopy for retinal chromophores: a) 11-cis from 2006 [46]; b) all-trans from 2006 [46]; c) all-trans from 2010 [47]. 
In the same experimental study [46], the authors also report a photo-dissociation spectrum for a different retinal conformer, the all-trans retinal chromophore. As we can see in Figure 1.6b, this spectrum is also rather broad with a main peak at about $2.0 \mathrm{eV}$ and two additional shoulders, one extending at higher energies. Theoretically, we find that both conformers have a very similar vertical excitation energy of about $2.3 \mathrm{eV}$. Consequently, the similar location of the absorption maximum in both spectra would seem to consistently indicate either that we are too blue shifted with respect to experiments or that vibronic effects are responsible for a similar red shift of the maximum with respect to the theoretical vertical excitation. Surprisingly, in a more recent study [47], the same experimental group has however produced another dissociation spectra for the all-trans retinal with a totally different shape, as show in Figure 1.6c. The new spectrum shows no structure but only a very flat plateau extending over a wide range of wavelengths (from 530 to $610 \mathrm{~nm}$ ). Not surprisingly, the new spectrum was promptly explained in terms of thermal effects with the help of calculations of the low-correlation type so commonly used for retinal. The unusually broad features of the spectrum were interpreted as due to the rotation of the $\beta$-ionone ring at room temperature between different conformers of retinal characterized by different excitation energies.

In Chapter 5, we extensively investigate this scenario by combining ab initio molecular dynamics simulations at room temperature with highly-correlated methods to refine the ground-state potential energy surface and the corresponding excitation energies. Our calculations provide compelling evidence that thermal effect cannot be responsible for the broad plateau observed experimentally. While it is unclear why so different spectra have been reported by the same group for the same system, we note that, also for Green Fluorescent Protein, there exist multiple experimental photo-dissociation spectra with significantly different spectral shapes [48-51]. It is also important to stress that dissociation spectroscopy does not directly measure the optical absorption spectrum but rather the yield of photofragments resulting from the electronic excitation the chromophore. Moreover, these experiments appear to suffer from potential complications such as the possible presence of multi-photon dissociation channels and the consequent non-trivial dependence of the shape of the spectrum on the excitation laser power. Consequently, it is an open question (also actively investigated by some experimental groups) whether these model experiments are representative of the optical absorption of a given molecule. Our findings indicate that the available spectra are not representative of the optical spectrum of retinal in the gas phase and call for further experimental characterization of the dissociation spectra. Finally, we want to stress that photo-dissociation experiments with their uncertain interpretation has been rather harmful for the field of theoretical photochemistry since these photo-dissociation spectra are available for several relevant biological chromophores (e.g. retinal, Green Fluorescent Protein, Photoactive Yellow Protein, DsRed) and are routinely used to benchmark different theoretical excited-state methods and establish their relative accuracy.

Having calibrated our theoretical description of the structural model and excitations of retinal in the gas phase, we can now introduce the protein environment 


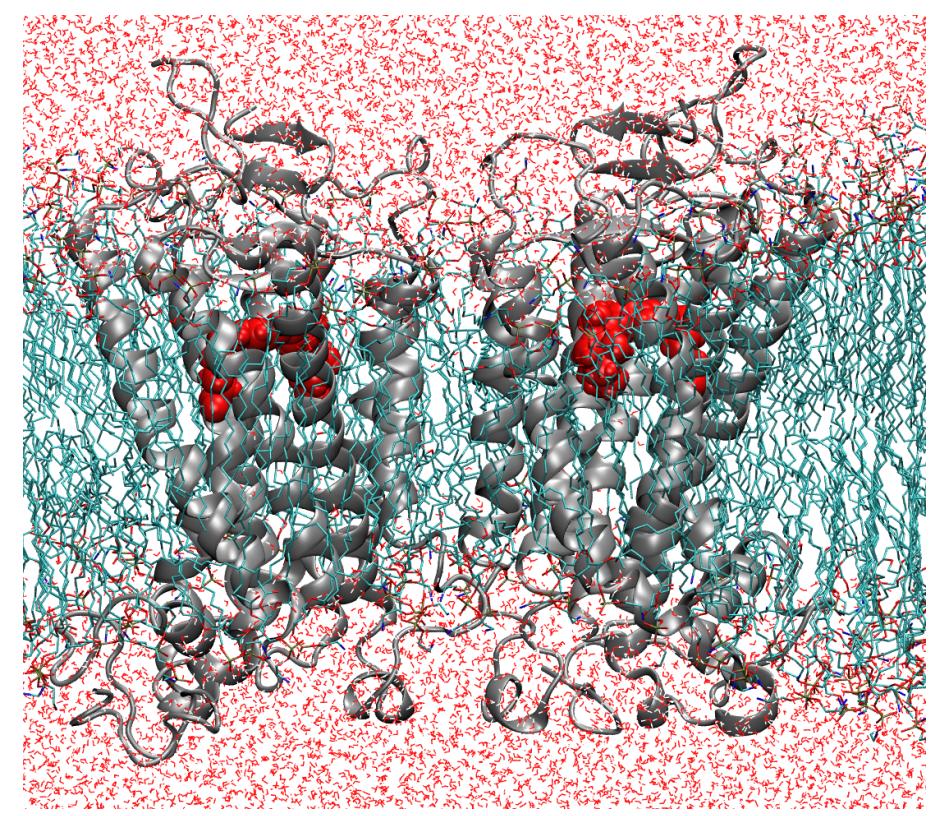

Figure 1.7: Rhodopsin dimer embedded in its native membrane from our simulations.

and consider the absorption of Rhodopsin, which is the focus of Chapter 6. As shown in Figure 1.7, we construct a realistic model of a Rhodopsin dimer embedded in its native membrane environment and consider the dynamical nature of the chromophore-protein system by performing extensive quantum in classical molecular dynamics simulations at room temperature. The availability of these trajectories allow us to compute the excitation energies over a large set of representative snapshots of the system instead of using models which are either rather close or even equal to a crystallographic structure from the Protein Data Bank as often done in previous studies of Rhodopsin. One of the main conclusions from our investigation is that the use of a classical description of the protein environment as in common quantum mechanical in molecular mechanics calculations is not adequate for retinal and leads to too high excitation energies. If the quantum region is enlarged to include a substantial number of amino acids in the surroundings of the chromophore, the protein environment responds to the excitation of the retinal chromophore, and the corresponding excitation red-shifts in the direction of the experimental absorption maximum of Rhodopsin. Naturally, a larger quantum region of 250 atoms is computationally very costly when we employ density-functional-based approaches, and is prohibitive for the highly-correlated excited-state methods we consider reliable for retinal. Therefore, we cannot directly estimate the shift we would obtain with an enlarged quantum region using these more accurate approaches, and whether it would be sufficient to bring their excitations in agreement with the absorption maximum in Rhodopsin. But then, should we obtain a perfect agreement with the experimental absorption maximum? A comparison of the vertical excitation with the experimental 
absorption maximum is what is commonly done by theoreticians for most systems, and so often a perfect agreement has in fact been claimed for Rhodopsin. However, should the Franck-Condon principle apply for the photo-active retinal system?

\section{a)}

$$
\text { Room Temp. ( }(\mathrm{T}=293 \mathrm{~K})
$$

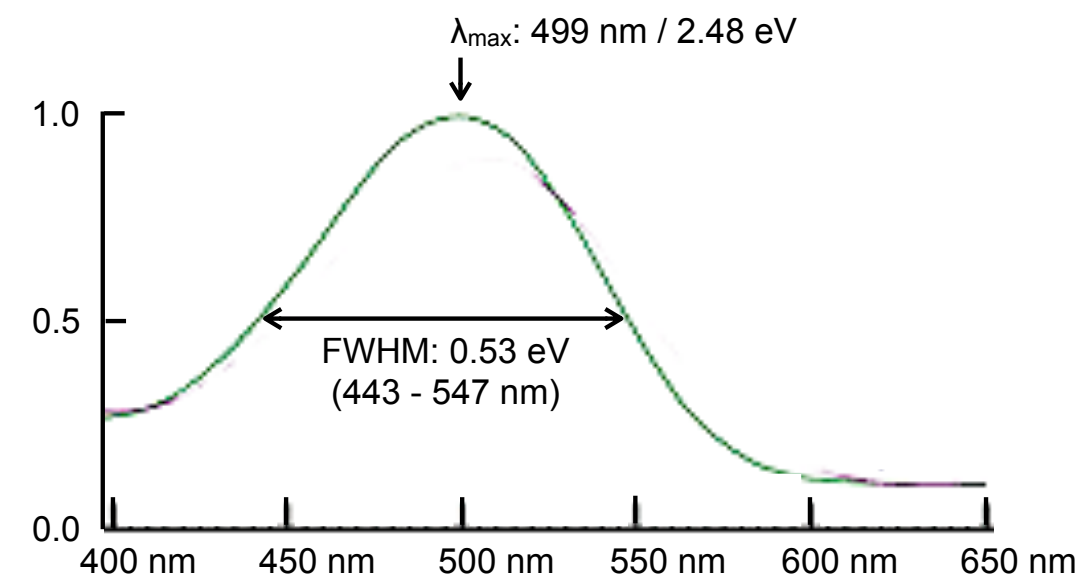

b)

$$
\text { Low Temp. }(\mathrm{T}=10 \mathrm{~K})
$$

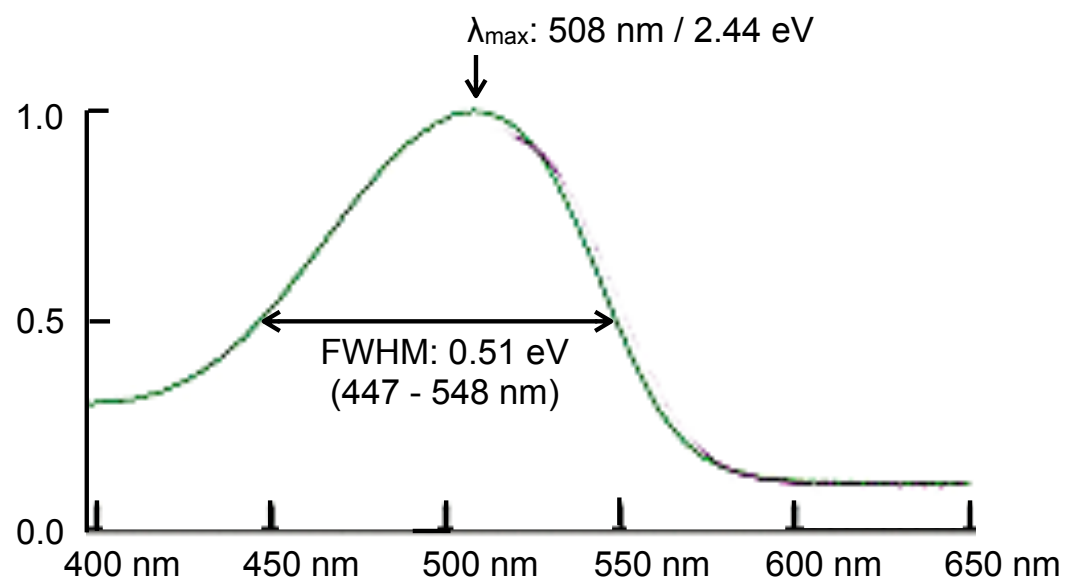

Figure 1.8: The experimental absorption spectra for Rhodopsin obtained at a) room temperature $(T=293 \mathrm{~K})$, and $\mathrm{b})$ low temperature $(T=10 \mathrm{~K})$. Figures adapted from Ref. 41.

Let us summarize what we have done in our theoretical study on the Rhodopsin absorption. With considerable effort, we have constructed a realistic structural model and included temperature effects. We have computed accurate excitation energies with a quantum in classical description to discover that the response of the protein must be accounted for beyond a simple classical representation of the environ- 
ment. If we were able to perform highly-correlated calculations with large quantum regions, we can infer that we would still be blue-shifted with respect to the absorption maximum of Rhodopsin by $0.1-0.2 \mathrm{eV}$. Is this finding reasonable? In Figures 1.8, we show the experimental absorption spectra for Rhodopsin obtained at room and low $(10 \mathrm{~K})$ temperature. Both absorption spectra are unstructured and very broad, and lowering the temperature has practically no effect. Therefore, the Franck-Condon envelope might be quite complicated and the vibronic effects large, especially since the chromophore undergoes ultra-fast isomerization upon photoexcitation. A disagreement of $0.1-0.2 \mathrm{eV}$ between the theoretical vertical excitation and the location of the absorption maximum can therefore be expected. With certainty, we can say that it is important to construct a realistic structural model of the photo-biological system and that more accurate (but more costly) schemes than a classical, non-polarizable treatment of the protein must be employed to deal with the chromophore-protein interaction in the computation of the excitation energies. As for the common habit of comparing the theoretical vertical excitation energy with the experimental absorption maximum, it does not not seem to be valid for Rhodopsin.

Here is where the story regarding the absorption of the retinal chromophore and Rhodopsin ends. Both in the gas phase and in the protein environment, we have carefully calibrated our theoretical tools and believe that the procedure we employ gives an accurate description of the vertical excitation energies of the system. Ultimately, the definite verdict on our theoretical procedure must come from a comparison with experiments. However, as it emerges from this thesis, such a comparison is not always clear-cut. For the retinal chromophore in the gas phase, our findings raise severe doubts on the available photo-dissociation spectroscopy experiments being representative of the optical absorption of retinal. For Rhodopsin, it appears that we (and others) should not compare theoretical vertical excitation energies with the experimental absorption maximum, given the rather large spectral broadening observed in experiments.

In Chapter 3, we also explore another important aspect of the visual process, namely, how the structural relaxation in the excited state proceeds upon photoexcitation. This theme is also ultimately related to our ability to compute a theoretical absorption spectrum for retinal and Rhodopsin, and move beyond vertical excitation energies, which are not what is measured in experiments anyhow. To compute a spectrum, we need to be able to relax the system in the excited state and therefore posses sufficiently accurate and efficient excited-state gradients. Here, we have taken the first steps in the investigation of this issue for simple retinal models in the gas phase. In the gas phase, solution, and protein, the widely accepted photoisomerization mechanism from the 11-cis to the all-trans retinal conformer is one of bond inversion followed by torsion around formal double bonds. For retinal in the gas phase, we predict instead a very different picture with the use of highlycorrelated approaches also in the computation of the excited-state interatomic forces. We find that the photo-excited chromophore is very flexible and essentially all bonds are active, with some torsions leading to photoisomerization and others along non- 
reactive paths. Our findings are compatible with solution experiments which indicate the existence of multiple minima and relaxation pathways, some of which are non-reactive and do not lead to photoproducts via conical intersection. It would have surely been interesting to attempt to investigate the photoisomerization process in the protein. However, determining the necessary ingredients for an accurate description of Rhodopsin absorption alone was already a tour de force, so the even more demanding task of relaxing the system in the excited state will be left to my (brave!) successor.

Finally, in Chapter 7, we digress from retinal and consider a different class of photosensitive molecules, the so-called cyanine dyes. This class of molecules has always been considered an intriguing and problematic case for excited-state density functional theory and a challenge for the development of new density functionals. We demonstrate that this belief is wrongly based on the use of flawed benchmark excitation energies, and offer carefully computed values as aid for future developments.

\subsection{Prospects}

One of the important conclusions of our Rhodopsin investigation is that the commonly used classical description of the protein environment does not yield sufficiently accurate excitation energies of the chromophore-protein system. The use of a larger quantum cluster would allow us to obtain more accurate excitations but the number of atoms we need to include in the quantum region becomes too large for the use of highly-correlated approaches. Therefore, it would be desirable to employ an improved description of the protein environment without loosing the multiscale partition of the system in an active region, to be treated at a higher computational level, and the rest of the protein.

We have preliminarily explored an interesting option, which we describe briefly here and which will require further investigation, namely, the use of subsystem density functional theory [52-54] to describe the protein environment. The idea is to construct a realistic representation of the environment with the use of density functional theory. In this approach, one obtains an effective potential of the protein, which depends on its ground-state electronic density and can then be combined with highly-correlated approaches for the computation of the excitation energies of the photo-sensitive quantum region. The quantum in classical description is therefore replaced by a quantum in quantum multiscale scheme. This approach will give a more accurate description of the protein pocket as we are now using a realistic electronic density instead of fixed, classical point charges to represent the amino acids surrounding the chromophore.

To generate this effective potential acting on the retinal chromophore, we must obtain the electronic density of the complete system and this is achieved by partitioning Rhodopsin into distinct regions as shown in Figure 1.9. Within subsystem density functional theory, we then converge the density of the each region in the 


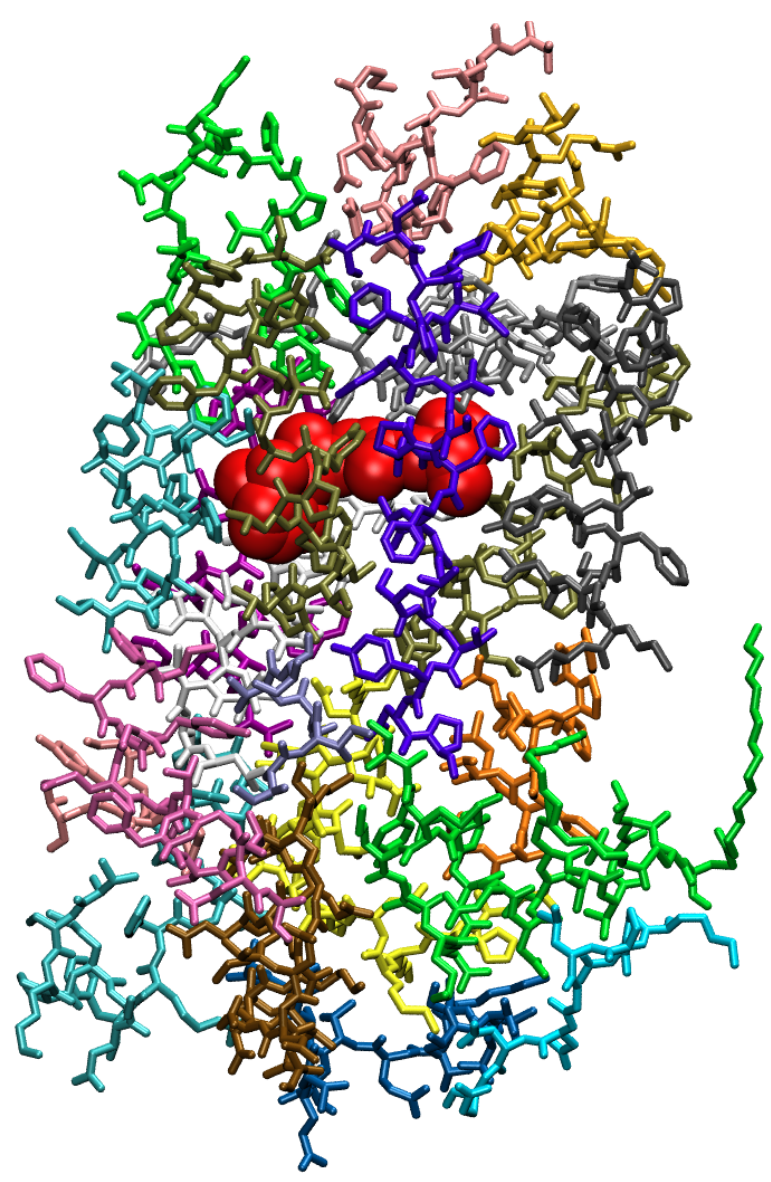

Figure 1.9: The partition of Rhodopsin into distinct regions is represented by different colors, and is used in the subsystem density functional theory calculations for a quantum in quantum multiscale treatment.

presence of the rest of the system, so each subsystem is polarized by the others. To explore the performance of this quantum in quantum scheme for Rhodopsin, we consider a configuration of the protein that gives a very high excitation energy when computed with the standard quantum in classical approach. Rather surprisingly, even though this effective environment surely represents a considerable improvement with respect to a classical description, we obtain exactly the same excitation energy as before.

We know however that increasing the size of the quantum region leads to a red shift of the excitation energy. Therefore, there are two other possible factors we need to account for: i) We need to allow the the protein environment to respond to the excitation of the chromophore; ii) the excitation is characterized by charged transfer between the chromophore and other amino acids, and it is not possible to partition the system in chromophore and the rest, so an enlarged quantum region is the only possible solution. Our calculations of Chapter 6 on large quantum clusters with excited-state density functional theory seem to rule out the second possibility 
for Rhodopsin since the excitation does not lead to charge transfer. Therefore, we can still describe our system as partitioned in an active region and the rest of the protein, and proceed with including "back-polarization" effects of the protein due to the photo-excitation of the chromophore.

Following this idea, we have generated an effective potential acting on the active chromophore, where the density of the rest of the protein is still relaxed in the ground state but in the presence of an excited chromophore. However, a straightforward use of such a "back-polarized" potential for the computation of the excited state of the chromophore presents several conceptual problems. We have recently addressed these difficulties through a proper formulation of a quantum in quantum scheme where different effective potentials are employed to compute the ground and the excited state of the active region [55]. The performance of the approach on the excitation energies of a small benchmark system (i.e. $p$-nitroaniline in water) is very promising since the scheme generally leads to excitation energies closer to the supermolecular values obtained for the whole system. The scheme will be applied to Rhodopsin in the near future, with the expectation that it will allow us to obtain accurate excitation energies with the use of relatively small quantum regions.

\subsection{Bibliography}

[1] G. Wald, Science 162, 230 (1968).

[2] J. Nathans, D. Thomas, and D. S. Hogness, Science 232, 193 (1986).

[3] Y. Shichida and H. Imai, Cell. Mol. Life. Sci. 54, 1299 (1998).

[4] J. Nathans, Neuron 24, 299 (1999).

[5] G. G. Kochendoerfer, S. W. Lin, T. P. Sakmar, and R. A. Mathies, Trend. Biochem. Sci. 24, 300 (1999).

[6] Handbook of Biological Physics: Molecular Mechanisms in Visual Transduction (Elsevier BV, 2000), Vol. 3.

[7] D. Hunt, S. Wilkie, J. Bowmaker, and S. Poopalasundaram, Cell. Mol. Life. Sci. 58, 1583 (2001).

[8] H. Kandori, Y. Shichida, and T. Yoshizawa, Biochemistry (Moscow) 66, 1197 (2001).

[9] T. Ebrey and Y. Koutalos, Prog. Retinal Eye Res. 20, 49 (2001).

[10] M. Burns and T. Lamb, in Visual Neurosciences, edited by L. Chalupa and J. Werner (MIT Press, 2003), pp. 215-233.

[11] J. K. Bowmaker and D. M. Hunt, Curr. Biol. 16, R484 (2006). 
[12] B. Nickle and P. R. Robinson, Cell. Mol. Life. Sci. 64, 2917 (2007).

[13] K. D. Ridge and K. Palczewski, J. Biol. Chem. 282, 9297 (2007).

[14] Y. Shichida and T. Morizumi, Photochem. Photobiol. 83, 70 (2007).

[15] S. Yokoyama, Annu. Rev. Genom. 9, 259 (2008).

[16] Y. Shichida and T. Matsuyama, Phil. Trans. R. Soc. B 364, 2881 (2009).

[17] M. B. Nielsen, Chem. Soc. Rev. 38, 913 (2009).

[18] C.-H. Sung and J.-Z. Chuang, J. Cell. Biol. 190, 953 (2010).

[19] K. Tsutsui and Y. Shichida, Photochem. Photobiol. Sci. 9, 1426 (2010).

[20] K. Palczewski, J. Biol. Chem. 287, 1612 (2012).

[21] V. J. Kefalov, J. Biol. Chem. 287, 1635 (2012).

[22] R. E. Stenkamp, S. Filipek, C. Driessen, D. Teller, and K. Palczewski, Biochim. Biophys. Acta 1565, 168 (2002).

[23] S. Filipek, R. E. Stenkamp, D. C. Teller, and K. Palczewski, Annu. Rev. Physiol. 65, 851 (2003).

[24] R. E. Stenkamp, D. C. Teller, and K. Palczewski, Arch. Pharm. Pharm. Med. Chem. 338, 209 (2005).

[25] K. Palczewski, Annu. Rev. Biochem. 75, 743 (2006).

[26] S. O. Smith, Annu. Rev. Biophys. 39, 309 (2010).

[27] K. Palczewski, T. Kumasaka, T. Hori, C. A. Behnke, H. Motoshima, B. A. Fox, I. L. Trong, D. C. Teller, T. Okada, R. E. Stenkamp, M. Yamamoto, and M. Miyano, Science 289, 739 (2000).

[28] D. C. Teller, T. Okada, C. A. Behnke, K. Palczewski, and R. E. Stenkamp, Biochemistry 40, 7761 (2001).

[29] T. Okada, Y. Fujiyoshi, M. Silow, J. Navarro, E. M. Landau, and Y. Shichida, Proc. Natl. Acad. Sci. U.S.A. 99, 5982 (2002).

[30] T. Okada, M. Sugihara, A.-N. Bondar, M. Elstner, P. Entel, and V. Buss, J. Mol. Biol. 342, 571 (2004).

[31] Obtained from Wikimedia commons:

"http://commons.wikimedia.org/wiki/File:Cone_scheme_ru.svg".

[32] T. Andruniów, N. Ferré, and M. Olivucci, Proc. Natl. Acad. Sci. U.S.A. 101, 17908 (2004). 
[33] M. Hoffmann, M. Wanko, P. Strodel, P. H. König, T. Frauenheim, K. Schulten, W. Thiel, E. Tajkhorshid, and M. Elstner, J. Am. Chem. Soc. 128, 10808 (2006).

[34] S. Sekharan, M. Sugihara, and V. Buss, Angew. Chem. Int. Ed. 46, 269 (2007).

[35] A. Strambi, P. B. Coto, N. Ferré, and M. Olivucci, Theor. Chem. Acc. 118, 185 (2007).

[36] K. Bravaya, A. Bochenkova, A. Granovsky, and A. Nemukhin, J. Am. Chem. Soc. 129, 13035 (2007).

[37] K. Fujimoto, S. Hayashi, J.-y. Hasegawa, and H. Nakatsuji, J. Chem. Theory Comput. 3, 605 (2007).

[38] A. Altun, S. Yokoyama, and K. Morokuma, J. Phys. Chem. B 112, 16883 (2008).

[39] G. Tomasello, G. Olaso-González, P. Altoeà, M. Stenta, L. Serrano-Andreás, M. Merchaán, G. Orlandi, A. Bottoni, and M. Garavelli, J. Am. Chem. Soc. 131, 5172 (2009).

[40] J. S. Frähmcke, M. Wanko, P. Phatak, M. A. Mroginski, and M. Elstner, J. Phys. Chem. B 114, 11338 (2010).

[41] M. N. Sandberg, T. L. Amora, L. S. Ramos, M.-H. Chen, B. E. Knox, and R. R. Birge, J. Am. Chem. Soc. 133, 2808 (2011).

[42] P. B. Coto, A. Strambi, N. Ferré, and M. Olivucci, Proc. Natl. Acad. Sci. U.S.A. 103, 17154 (2006).

[43] K. Fujimoto, J.-Y. Hasegawa, and H. Nakatsuji, Chem. Phys. Lett. 462, 318 (2008).

[44] F. Melaccio, N. Ferré, and M. Olivucci, Phys. Chem. Chem. Phys. doi: 10.1039/c2cp40940b (2012).

[45] J. S. Frähmcke, M. Wanko, and M. Elstner, J. Phys. Chem. B 116, 3313 (2012).

[46] I. B. Nielsen, L. Lammich, and L. H. Andersen, Phys. Rev. Lett. 96, 018304 (2006).

[47] J. Rajput, D. Rahbek, L. Andersen, A. Hirshfeld, M. Sheves, P. Altoè, G. Orlandi, and M. Garavelli, Angew. Chem. Int. Ed. 49, 1790 (2010).

[48] S. B. Nielsen, A. Lapierre, J. U. Andersen, U. V. Pedersen, S. Tomita, and L. H. Andersen, Phys. Rev. Lett. 87, 228102 (2001).

[49] M. W. Forbes and R. A. Jockusch, J. Am. Chem. Soc. 131, 17038 (2009). 
[50] K. Chingin, R. M. Balabin, V. Frankevich, K. Barylyuk, R. Nieckarz, P. Sagulenko, and R. Zenobi, Int. J. Mass Spectrom. 306, 241 (2011).

[51] M. W. Forbes, A. M. Nagy, and R. A. Jockusch, Int. J. Mass Spectrom. 308, 155 (2011).

[52] T. A. Wesolowski and A. Warshel, J. Phys. Chem. 97, 8050 (1993).

[53] A. S. P. Gomes, C. R. Jacob, and L. Visscher, Phys. Chem. Chem. Phys. 10, 5353 (2008).

[54] J. Neugebauer, Phys. Rep. 489, 1 (2010).

[55] C. Daday, O. Valsson, C. König, C. Filippi, and J. Neugebauer, Submitted (2012). 


\section{Chapter 2}

\section{Theoretical Methods}

\subsection{Introduction}

To investigate the photophysics of retinal in the gas phase as well as in the protein environment of Rhodopsin, we will employ a variety of computational methods since the problem is characterized by different spacial and temporal scales and cannot be addressed with the use of a single theoretical approach. The assessment of the performance/reliability of the excited-state approaches has been a critical aspect of our investigation as our findings clash against the results of a decade of main-stream calculations on retinal systems. The extent of this effort is reflected in the particularly large number of theoretical methods employed for the quantum mechanical description of the photo-excitations in retinal.

In this chapter, we give a short description of the various methods, which range from classical molecular dynamics to highly-correlated many-body techniques, and begin below with the latter. We describe in particular the multi-configuration selfconsistent (MCSCF) approach and its perturbation corrections for the computation of excited states, the continuous quantum Monte Carlo methods, and time-dependent density functional theory (TDDFT). We then outline how to combine these quantum mechanical methods with classical molecular mechanics (QM/MM) to treat the chromophore embedded in a larger classical system.

\subsection{Quantum Mechanical Calculations}

We work here in the Born-Oppenheimer approximation [1,2], and neglect relativistic effects so that our non-relativistic system of $N$ interacting electrons is described by the Hamiltonian:

$$
\mathcal{H}=-\frac{1}{2} \sum_{i=1}^{N} \nabla_{i}^{2}+\sum_{i=1}^{N} v_{\text {ext }}\left(\mathbf{r}_{\mathbf{i}}\right)+\sum_{i<j}^{N} \frac{1}{\left|\mathbf{r}_{i}-\mathbf{r}_{j}\right|},
$$

where we used atomic units ( $\hbar=m=e=1)$. The external potential is either the bare electron-ion Coulomb potential $-Z / r$ where $Z$ is the charge of the ion, 
or a pseudopotential describing the ion plus the core electrons which have been eliminated from the calculation. We denote with $\mathrm{x}=(\mathbf{r}, \sigma)$ the 3 spacial and 1 spin coordinates of one electron where $\sigma= \pm 1$.

\subsubsection{Traditional Quantum Chemistry Methods}

The simplest approach for the description of a system of $N$ interacting electrons and the starting point for the construction of more complex many-body wave functions is the the Hartree-Fock (HF) method $[1,2]$. In this approach, one describes the interacting system with the optimal non-interacting wave function, namely, a Slater determinant of single-particle spin-orbitals $\left\{\Phi_{i}\right\}$

$$
\Psi_{\mathrm{HF}}\left(\mathbf{x}_{1}, \ldots, \mathbf{x}_{N}\right)=\frac{1}{\sqrt{N !}}\left|\begin{array}{cccc}
\Phi_{1}\left(\mathbf{x}_{1}\right) & \Phi_{1}\left(\mathbf{x}_{2}\right) & \ldots & \Phi_{1}\left(\mathbf{x}_{N}\right) \\
\Phi_{2}\left(\mathbf{x}_{1}\right) & \Phi_{2}\left(\mathbf{x}_{2}\right) & \ldots & \Phi_{2}\left(\mathbf{x}_{N}\right) \\
\vdots & \vdots & \vdots & \vdots \\
\Phi_{N}\left(\mathbf{x}_{1}\right) & \Phi_{N}\left(\mathbf{x}_{2}\right) & \cdots & \Phi_{2}\left(\mathbf{x}_{N}\right)
\end{array}\right|
$$

where the single-particle orbitals are determined by minimizing the expectation value of the interacting Hamiltonian on this wave function. By expressing the spinorbitals as the product of a spacial and a spin components, $\Phi_{i}(\mathbf{x})=\phi_{i}(\mathbf{r}) \chi_{s_{i}}(\sigma)$ and minimizing the energy subject to orthonormality constraint, one obtains that the spacial orbitals must satisfy the self-consistent HF equations:

$$
\begin{aligned}
& {\left[-\frac{1}{2} \nabla^{2}+v_{\text {ext }}(\mathbf{r})+\sum_{j=1}^{N} \int \mathrm{d} \mathbf{r}^{\prime} \frac{\left|\phi_{j}\left(\mathbf{r}^{\prime}\right)\right|^{2}}{\left|\mathbf{r}-\mathbf{r}^{\prime}\right|}\right] \phi_{i}(\mathbf{r})} \\
& -\sum_{j=1}^{N} \delta_{s_{i}, s_{j}} \int \mathrm{d} \mathbf{r}^{\prime} \frac{\phi_{j}^{*}\left(\mathbf{r}^{\prime}\right) \phi_{i}\left(\mathbf{r}^{\prime}\right)}{\left|\mathbf{r}-\mathbf{r}^{\prime}\right|} \phi_{j}(\mathbf{r})=\epsilon_{i} \phi_{i}(\mathbf{r}) .
\end{aligned}
$$

The non-local HF exchange potential cancels the interaction of the electron with itself, that is the self-interaction contribution from the the Hartree potential, and keeps the electrons of the same spin apart.

For molecular systems, the orbitals are expanded as a linear combination of atomic orbitals (LCAO) centered on the nuclear positions:

$$
\phi_{i}(\mathbf{r})=\sum_{\mu}^{\text {nuclei }} \sum_{j} a_{j i}^{\mu} \eta_{j \mu}\left(\mathbf{r}-\mathbf{r}_{\mu}\right),
$$

where $\mathbf{r}_{\mu}$ denotes the position of a nucleus, and the minimization is performed with respect to the LCAO coefficients, $a_{j i}^{\mu}$. In most quantum chemistry codes, a Gaussian atomic basis is used:

$$
\eta(\mathbf{r})=x^{m} y^{n} z^{k} \exp \left(-\alpha r^{2}\right),
$$

as this choice allows all integrals to be computed analytically.

The difference between the exact energy $E$ and the HF energy is called the correlation energy, $E_{\text {corr }}=E-E_{\mathrm{HF}}$. 


\section{Post Hartree-Fock Methods}

Traditional post-HF approaches rely implicitly or explicitly in expressing the manybody wave function, $\Psi\left(\mathbf{x}_{1}, \ldots, \mathbf{x}_{N}\right)$, as an expansion in the non-interacting basis of determinants of single-particle orbitals $[1,2]$. The matrix elements of the Hamiltonian and the overlap of these $N$-electron basis functions can then be computed analytically if the single-particle orbitals are expressed on an atomic Gaussian basis set.

In the configuration interaction (CI) approach, one constructs the correlated wave function by considering excitations out of the reference HF determinant to the set of virtual orbitals as

$$
\Psi_{\mathrm{CI}}=c_{0} D_{\mathrm{HF}}+\sum_{a b} c_{a \rightarrow b} D^{a \rightarrow b}+\sum_{a b c d} c_{a b \rightarrow c d} D^{a b \rightarrow c d}+\ldots
$$

where $D^{a \rightarrow b}$ denotes a single excitation where the occupied orbital $a$ in the HF determinant is substituted with the virtual orbital $b$. Similarly, $D^{a b \rightarrow c d}$ indicates a double excitation from $a$ and $b$ to the virtual orbitals $c$ and $d$. If we include up to $N$-body excitations to all virtual orbitals, we obtain the full CI expansion, which must then be extrapolated to infinite basis limit by considering a sequence of larger basis sets. If we denote with $C_{i}$ a spin- and space-adapted configuration state functions (CSF) (i.e. a fixed linear combination of determinants with proper spin and space symmetry), we can rewrite a CI expansion as

$$
\Psi_{\mathrm{CI}}=\sum_{i=1}^{K} c_{i} C_{i}
$$

and, by applying the variational principle, obtain the secular equations for the coefficients $c_{i}$ :

$$
\sum_{j=1}^{K}\left\langle C_{i}|\mathcal{H}| C_{j}\right\rangle c_{j}^{(k)}=E_{\mathrm{CI}}^{(k)} \sum_{j=1}^{K}\left\langle C_{i} \mid C_{j}\right\rangle c_{j}^{(k)},
$$

where $\left\langle C_{i} \mid C_{j}\right\rangle=\delta_{i j}$ as the orbitals are orthonormal.

For a CI expansion (and any linear expansion on a basis set), one obtains not only an approximation to the ground state wave function but also to the excited states thanks to a generalized variational principle, known as the the McDonald's theorem, which states that the approximate solutions with energies $E_{\mathrm{CI}}^{(0)} \leq E_{\mathrm{CI}}^{(1)} \leq \ldots \leq E_{\mathrm{CI}}^{(K)}$ satisfy

$$
E_{i} \leq E_{\mathrm{CI}}^{(i)}
$$

where $E_{i}$ are the exact energies of the eigenstates of the Hamiltonian $\mathcal{H}$. A CI wave function is however a slowly converging expansion and a large number of determinants must be included due to the lack of explicit dependence from the interelectron coordinates, and the consequent poor description of the cusp occurring at the electron-electron coalescence points. Moreover, the number of determinants grows exponentially with the number of electrons $N$ and, while limiting the number 
of determinants to the most important excitations lowers the computational cost (for instance, CISD includes single and doubles and scales as $N^{6}$ ), it results in the loss of size consistency.

In the multi-configuration self consistent field (MCSCF) approach, one minimizes the energy not only with respect to the linear coefficients $c_{i}$ but also the LCAO coefficients $a_{j i}$. The complete active space self-consistent (CASSCF) approach is a particular type of MCSCF calculation, where $n$ electrons are distributed over a set of $m$ active orbitals, whose occupancy is allowed to vary. The resulting $\operatorname{CASSCF}(n, m)$ calculation is like a full CI calculation for $n$ electrons in $m$ orbitals, except that also the orbitals are now optimized to minimize the total energy.

When several states of the same symmetry are requested, it is customary to use the state averaged (SA) CASSCF approach to avoid root-flipping problems in the optimization. In a SA calculations, the weighted average of the energies of the states of interest is optimized

$$
E_{\mathrm{SA}}=\sum_{I} w_{I} \frac{\left\langle\Psi_{I}|\mathcal{H}| \Psi_{I}\right\rangle}{\left\langle\Psi_{I} \mid \Psi_{I}\right\rangle},
$$

where $\sum_{I} w_{I}=1$ and the states are kept orthogonal. The optimization yields one common set of orbitals and the different states differ in their CI coefficients. Orthogonality is ensured via the CI coefficients and a generalized variational theorem applies. The most important step for a MCSCF/CASSCF calculation is the selection of the active space, which requires a fair amount of knowledge of the system under study and is rather time-consuming since a great number of trial calculations is often necessary. However, we note that a MCSCF/CASSCF calculations recover only a small part of the correlation energy as it only provides a description of the non-dynamical correlation.

One way to improve up on a MCSCF calculation is to use Rayleigh-Schrödinger perturbation theory, where the total Hamiltonian is partitioned into a zero-order Hamiltonian, $\mathcal{H}^{(0)}$, and a perturbing operator $\mathcal{V}$

$$
\mathcal{H}=\mathcal{H}^{(0)}+\mathcal{V} .
$$

The definition of the perturbation theory is complete once we have decided on the zero-order wave function and Hamiltonian. If we take the zero-order wave function to be the HF wave function, we would obtain Möller-Plessant perturbation theory (MP2). Taking a MCSCF wave function to be the zero-order wave function, we obtain multi-reference perturbation theory (MRPT). Specifically, in the case of a CASSCF wave function, this has resulted in complete active space perturbation theory (CASPT2) [3], which is one of the most widely used method in excited-state quantum chemistry However, for MRPT there is not an unique definition of the zero-order Hamiltonian and this has resulted in multiple formulations of CASPT2, which differ in the choice of the zero-order Hamiltonian. More recent MRPT that is also based on a CASSCF zero-order wave function is the so-called $n$-electron valence state perturbation theory (NEVPT2) [4], which as compared to CASPT2 is based on a more advanced zero-order Hamiltonians. The important difference is 
that zero-order Hamiltonian in NEVPT2 explicitly includes two-electron terms for the active electrons, while the CASPT2 zero-order Hamiltonian only includes oneelectron terms. In Chapter 4 of this thesis, we explore how the different choices of the zero-order Hamiltonian in CASPT2 affect the excitation energies of retinal and, furthermore, how NEVPT2 compares to CASPT2.

\subsubsection{Density Functional Theory}

Density functional theory (DFT) [5] represents a very appealing alternative to traditional quantum chemistry approaches because of its simplicity and computational efficiency. In DFT, one abandons a wave function description of the system of interacting electrons, and the ground-state energy is expressed as a functional of the ground-state electronic density [6]. In the Kohn-Sham formulation of density functional theory [7], the ground state density is then written in terms of single-particle orbitals obeying the equations:

$$
\left[-\frac{1}{2} \nabla^{2}+v_{\mathrm{eff}}([n] ; \mathbf{r})\right] \phi_{i}=\epsilon_{i} \phi_{i}
$$

where the electronic density is constructed by summing over the $N$ lowest energy orbitals where $N$ is the number of electrons:

$$
n(\mathbf{r})=\sum_{i=1}^{N}\left|\phi_{i}(\mathbf{r})\right|^{2}
$$

The effective Kohn-Sham potential is given by

$$
v_{\text {eff }}([n] ; \mathbf{r})=v_{\text {ext }}(\mathbf{r})+\int \frac{n\left(\mathbf{r}^{\prime}\right)}{\left|\mathbf{r}-\mathbf{r}^{\prime}\right|} \mathrm{d} \mathbf{r}^{\prime}+v_{\text {xc }}([n] ; \mathbf{r})
$$

$v_{\text {ext }}(\mathbf{r})$ is the external potential. The exchange-correlation potential $v_{\mathrm{xc}}([n] ; \mathbf{r})$ is the functional derivative of the exchange-correlation energy $E_{\mathrm{xc}}[n]$ that enters in the expression for the total energy:

$$
\begin{aligned}
E & =-\frac{1}{2} \sum_{i=1}^{N} \int \phi_{i} \nabla^{2} \phi_{i} \mathrm{~d} \mathbf{r}+\int n(\mathbf{r}) v_{\text {ext }}(\mathbf{r}) \mathrm{d} \mathbf{r} \\
& +\frac{1}{2} \iint \frac{n(\mathbf{r}) n\left(\mathbf{r}^{\prime}\right)}{\left|\mathbf{r}-\mathbf{r}^{\prime}\right|} \mathrm{d} \mathbf{r} \mathrm{d} \mathbf{r}^{\prime}+E_{\mathrm{xc}}[n] .
\end{aligned}
$$

Even though DFT is in principle exact, the exchange-correlation energy is an unknown functional of the density and must be approximated.

\section{Approximate Exchange-Correlation Functionals}

Several approximate exchange-correlation functionals have been proposed in the literature [8], and recent years have in fact seen a proliferation of novel functionals, 
sometimes designed for the study of specific properties. In this thesis, we employ generalized gradient approximation (GGA), hybrid, meta-hybrid, and long-range corrected (LC) functionals.

A "classical" functional form is the generalized gradient approximation (GGA), whose generic expression (here restricted to second-order derivatives) is given by

$$
E_{\mathrm{xc}}^{\mathrm{GGA}}[n]=\int n(\mathbf{r}) \epsilon_{\mathrm{xc}}^{\mathrm{GGA}}\left(n(\mathbf{r}),|\nabla n(\mathbf{r})|, \nabla^{2} n(\mathbf{r})\right) \mathrm{d} \mathbf{r} .
$$

We will use in particular the Becke-Lee-Yang-Parr (BLYP) $[9,10]$ and the PerdewBurke-Ehrennshof (PBE) [11] GGA functionals in the ground-state ab initio molecular dynamics simulations with the CPMD and CP2K codes. The GGA functional form is in fact a preferable choice for use in such plane-wave codes since more advanced functionals including exact exchange are too costly in a plane-wave formalism.

Hybrid functionals introduce a dependence on the Kohn-Sham orbitals by mixing in the functional a portion of exact exchange from Hartree-Fock theory:

$$
E_{\mathrm{x}}^{\mathrm{HF}}[n]=-\frac{1}{2} \sum_{i=1}^{N} \sum_{j=1}^{N} \delta_{s_{i}, s_{j}} \iint \frac{\phi_{i}^{*}(\mathbf{r}) \phi_{j}^{*}\left(\mathbf{r}^{\prime}\right) \phi_{j}(\mathbf{r}) \phi_{i}\left(\mathbf{r}^{\prime}\right)}{\left|\mathbf{r}-\mathbf{r}^{\prime}\right|} \mathrm{d} \mathbf{r} \mathrm{d} \mathbf{r}^{\prime} .
$$

A widely used hybrid functional is the three parameter B3LYP functional $[12,13]$ which combines LDA and the BLYP GGA with exact exchange. We employ the B3LYP functional in the ground-state optimization of retinal models within Gaussian.

Meta-GGA functional introduce dependence on the non-inteacting kinetic density

$$
\tau=\frac{1}{2} \sum_{i}\left|\nabla \phi_{i}(\mathbf{r})\right|^{2}
$$

Like a normal GGA, a meta-GGA can be combined with the inclusion of exact exchange, resulting in a meta-hybrid functional. A popular choice among these functionals is the M06 [14-17] family, a set of four functionals, each of which designed for a different purpose, that differ mainly in the amount of exact exchange included; M06-L (0\%), M06 (27\%), M06-2X (54\%), M06-HF (100\%). We employ these functionals in the ground-state optimization of retinal models within Gaussian, and in the subsystem-DFT calculations with ADF.

Finally, we consider the long-range corrected functionals (a good overview of them can be found in Ref. 18), where we split the electron-electron interaction into a short- and a long-range component

$$
\frac{1}{r}=\frac{1-g(r)}{r}+\frac{g(r)}{r}
$$

where, for instance, $g(r)=\operatorname{erf}(\mu r)$. The short-range component is treated through a density functional approximation while the long-range component is considered as exact exchange, resulting in

$$
E_{\mathrm{xc}}[n]=E_{\mathrm{x}}^{\mathrm{GGA}, \mathrm{SR}}[n]+E_{\mathrm{x}}^{\mathrm{HF}, \mathrm{LR}}[n]+E_{\mathrm{c}}^{\mathrm{GGA}}[n]
$$


The usage of the error function allows the exact exchange part to be obtained analytically while for the GGA density functional part an approximation based on a LDA ansatz is normally used $[8,19]$. As given above the long-range corrected functionals include $0 \%$ exact exchange at short range and $100 \%$ exact exchange at long range. However, the formulation can been generalized to include a certain amount of exact exchange at short range, for example by using $g(r)=\alpha+\beta \operatorname{erf}(\mu r)$ as done in the so-called CAM (Coulomb-attenuating method) functionals [20]. In particular, the popular CAM-B3LYP functional [20] includes $19 \%$ exact exchange at short range, although differently from most other long-range corrected functionals it only includes $65 \%$ exact exchange at long range. It has been observed that the long-range corrected functional can cure the well-known shortcoming of other functional forms to handle charge-transfer excitation within TDDFT. Here, we employ the CAM-B3LYP functional [20] and the LC- $\omega$ PBE [21,22] functional in the study of the excited states of retinal.

\section{Time-Dependent Density Functional Theory}

Time-dependent density-functional theory (TDDFT) [23] represents a rigorous formalism to compute excitation energies. As in the case of ground-state DFT, while exact in principle, TDDFT relies in practice on the use of approximate exchangecorrelation functionals.

The time-dependent equivalent of the Hohenberg-Kohn theorem is the RungeGross theorem [24]. It proves the one-to-one correspondence between the external time-dependent potential $v_{\text {ext }}(\mathbf{r}, \mathrm{t})$ and the time-dependent electronic density, $n(\mathbf{r}, \mathrm{t})$ and leads to the construction of a time-dependent Kohn-Sham scheme. The interating problem is then exactly mapped to a system of non-interacting electrons in an effective external time-dependent potential:

$$
\left[-\frac{1}{2} \nabla^{2}+v_{\text {eff }}([n] ; \mathbf{r}, t)\right] \phi_{i}(\mathbf{r}, t)=i \frac{\partial}{\partial t} \phi_{i}(\mathbf{r}, t),
$$

which yields the exact electronic density constructed from the Kohn-Sham orbitals as

$$
n(\mathbf{r}, t)=\sum_{i=1}^{N}\left|\phi_{i}(\mathbf{r}, t)\right|^{2}
$$

The Kohn-Sham effective potential is given by

$$
v_{\text {eff }}([n] ; \mathbf{r}, t)=v_{\text {ext }}(\mathbf{r}, t)+\int \frac{n\left(\mathbf{r}^{\prime}, t\right)}{\left|\mathbf{r}-\mathbf{r}^{\prime}\right|} \mathrm{d} \mathbf{r}^{\prime}+v_{\mathrm{xc}}([n] ; \mathbf{r}, t),
$$

Here, it is important to note that the time-dependent exchange-correlation potential is not the same functional of the density as the ground-state exchange-correlation potential (Eq. 2.13). Instead, it is the functional derivative of the exchange-correlation component of the action functional [24,25]. 
Some approximations are needed as the time-dependent exchange-correlation potential is unknown. The simplest approximation is to assume that the exchangecorrelation potential is local in time, that is, reacts instantaneously and without memory to any temporal change of the density. This is known as the adiabatic approximation:

$$
v_{\mathrm{xc}}^{\mathrm{adiab}}([n] ; \mathbf{r}, t)=\left.v_{\mathrm{xc}}^{\mathrm{gs}}([n] ; \mathbf{r})\right|_{n=n(\mathbf{r}, t)}
$$

where $v_{\mathrm{xc}}^{\mathrm{gs}}$ is a particular chosen ground-state exchange-correlation potential. Here, $v_{\mathrm{xc}}^{\mathrm{gs}}$ is a property of the ground-state, so this approximation should works best for time-dependent systems where the density does not deviated too far from the groundstate density.

If we know how the system responds to a small time-dependent perturbation, then the excitation energies can be easily obtained from a TDDFT calculation. To achieve this, the key quantity is the linear density response function $\chi$ that gives the change in the density if the system is subjected to a small perturbation in the external potential:

$$
\delta n_{\sigma}(\mathbf{r}, \omega)=\int \mathrm{d} \mathbf{r}^{\prime} \chi\left(\mathbf{r}, \mathbf{r}^{\prime}, \omega\right) \delta v_{\mathrm{ext}}\left(\mathbf{r}^{\prime}, \omega\right)
$$

Using the linear density response function, we can compute the dynamic polarizability and consequently obtain the photoabsorption cross section. We can rewrite this change in the density by using the time-dependent Kohn-Sham scheme (Eqs. 2.202.22), resulting in

$$
\delta n_{\sigma}(\mathbf{r}, \omega)=\int \mathrm{d} \mathbf{r}^{\prime} \chi_{\mathrm{KS}}\left(\mathbf{r}, \mathbf{r}^{\prime}, \omega\right) \delta v_{\mathrm{eff}}\left(\mathbf{r}^{\prime}, \omega\right)
$$

Here, $\chi_{\mathrm{KS}}$ is the density response function for the non-interacting Kohn-Sham electron system, which we can write in the terms of the unperturbed time-independent Kohn-Sham orbitals. We can obtain the linear change in the potential if we make use of the definition of the exchange-correlation potential (Eq. 2.21)

$$
\delta v_{\mathrm{eff}}(\mathbf{r}, \omega)=\delta v_{\mathrm{ext}}(\mathbf{r}, \omega)+\int \mathrm{d} \mathbf{r}^{\prime}\left[\frac{1}{\left|\mathbf{r}-\mathbf{r}^{\prime}\right|}+f_{\mathrm{xc}}\left(\mathbf{r}, \mathbf{r}^{\prime}, \omega\right)\right] \delta n\left(\mathbf{r}^{\prime}, \omega\right)
$$

Here, $f_{\mathrm{xc}}\left([n] ; \mathbf{r}, \mathbf{r}^{\prime}, \omega\right)$ is the Fourier transform of the exchange-correlation kernel:

$$
f_{\mathrm{xc}}\left([n] ; \mathbf{r}, \mathbf{r}^{\prime}, t-t^{\prime}\right)=\frac{\delta v_{\mathrm{xc}}([n] ; \mathbf{r}, t)}{\delta n\left(\mathbf{r}^{\prime}, t^{\prime}\right)} .
$$

By combining Eqs. 2.24-2.26, we can derive a Dyson-like equation for the response function

$$
\begin{aligned}
\chi\left(\mathbf{r}, \mathbf{r}^{\prime}, \omega\right) & =\chi_{\mathrm{KS}}\left(\mathbf{r}, \mathbf{r}^{\prime}, \omega\right) \\
& +\int \mathrm{d} \mathbf{x} \int \mathrm{d} \mathbf{x}^{\prime} \chi(\mathbf{r}, \mathbf{x}, \omega)\left[\frac{1}{\left|\mathbf{x}-\mathbf{x}^{\prime}\right|}+f_{\mathrm{xc}}\left(\mathbf{x}, \mathbf{x}^{\prime}, \omega\right)\right] \chi_{\mathrm{KS}}\left(\mathbf{x}^{\prime}, \mathbf{r}^{\prime}, \omega\right)
\end{aligned}
$$


The response $\chi$ of the interacting system can be obtained via a self-consistent solution if the exact exchange-correlation kernel is known. However, achieving a complete numerical solution of this equation is rather difficult. Instead, we make use of the knowledge that the excitation energies of the interacting system correspond to the poles of the density response function $\chi$. In the same manner, the Kohn-Sham response function $\chi_{\mathrm{KS}}$ has poles that coincide with the non-interacting excitation energies, which are given by the difference of Kohn-Sham eigenvalues.

By employing a sequence of algebraic manipulations, linear-response TDDFT can be expressed in the form of so-called Casida's equations [26], which is form used in most quantum chemistry codes. There, the poles of the the response functions, $\Omega=E_{m}-E_{0}$, are obtained as solutions of a non-Hermitian eigenvalue problem:

$$
\left[\begin{array}{ll}
\mathbf{A} & \mathbf{B} \\
\mathbf{B} & \mathbf{A}
\end{array}\right]\left(\begin{array}{c}
\vec{X} \\
\vec{Y}
\end{array}\right)=\Omega\left[\begin{array}{cc}
-\mathbf{1} & \mathbf{0} \\
\mathbf{0} & \mathbf{1}
\end{array}\right]\left(\begin{array}{c}
\vec{X} \\
\vec{Y}
\end{array}\right)
$$

where the matrices $\mathbf{A}$ and $\mathbf{B}$ are defined as

$$
\begin{aligned}
& A_{i a, i^{\prime} a^{\prime}}=\delta_{i i^{\prime}} \delta_{a a^{\prime}}\left(\epsilon_{a}-\epsilon_{i}\right)+K_{i a, i^{\prime} a^{\prime}} \\
& B_{i a, i^{\prime} a^{\prime}}=K_{i a, a^{\prime} i^{\prime}}=\left(i a\left|\frac{1}{\left|\mathbf{r}-\mathbf{r}^{\prime}\right|}\right| a^{\prime} i^{\prime}\right)+\left(i a\left|f_{\mathrm{xc}}\right| a^{\prime} i^{\prime}\right) .
\end{aligned}
$$

The excitation energies are given by the eigenvalues, while the oscillator strengths can be computed from the eigenvectors.

TDDFT is a very appealing method for obtaining the excited-state properties of large molecular systems as it has a rather favorable scaling of approximately $O\left(N^{3}\right)$ and is often fairly accurate. Nevertheless, it is important to note that linear-response TDDFT does have some limits [23], which one has to be aware of. For example, it only contains dressed one-electron excitations, which is a problem if the system under study displays multi-configurational character. Another well-known problem is the underestimation of charge transfer excitations [27-29]. However, the rangeseparated functionals seem to provide an elegant solution to this problem.

\subsubsection{Quantum Monte Carlo Methods}

We present here the variational (VMC) and diffusion (DMC) Monte Carlo methods, which are the most commonly used continuous quantum Monte Carlo (QMC) [30] techniques. These methods are wave function based but differ from conventional quantum chemistry as they attempt to solve the Schrödinger equation stochastically. Since the integrals are computed stochastically (and not analytically as in other quantum chemical methods), they offer significantly more freedom in the choice of the functional form of the many-body wave function. Moreover, both approaches scale rather favorably with the number of electrons, namely, $N^{4}$ as compared for instance to $N^{7}$ of the coupled cluster single and double with perturbative triples method. 


\section{Variational Monte Carlo}

The variational Monte Carlo method is the simplest flavor of QMC and uses Monte Carlo techniques to evaluate the expectation value of an operator on a given wave function. For instance, let us assume that we are interested in computing the expectation value of the Hamiltonian $\mathcal{H}$ on a given trial wave function $\Psi_{\mathrm{T}}$ :

$$
E_{V}=\frac{\int \Psi_{\mathrm{T}}^{*}(\mathbf{R}) \mathcal{H} \Psi_{\mathrm{T}}(\mathbf{R}) d \mathbf{R}}{\int \Psi_{\mathrm{T}}^{*}(\mathbf{R}) \Psi_{\mathrm{T}}(\mathbf{R}) \mathrm{d} \mathbf{R}}
$$

where we denote with $\mathbf{R}$ the $3 \mathrm{~N}$ electron coordinates. This expectation value can be rewritten as

$$
E_{V}=\frac{\int\left|\Psi_{\mathrm{T}}(\mathbf{R})\right|^{2}\left[\Psi_{\mathrm{T}}(\mathbf{R})^{-1} \mathcal{H} \Psi_{\mathrm{T}}(\mathbf{R})\right] d \mathbf{R}}{\int\left|\Psi_{\mathrm{T}}(\mathbf{R})\right|^{2} d \mathbf{R}}=\int \rho(\mathbf{R}) E_{L}(\mathbf{R}) \mathrm{d} \mathbf{R}
$$

where

$$
\rho(\mathbf{R})=\frac{\left|\Psi_{\mathrm{T}}(\mathbf{R})\right|^{2}}{\int\left|\Psi_{\mathrm{T}}(\mathbf{R})\right|^{2} \mathrm{~d} \mathbf{R}},
$$

and the local energy is defined as

$$
E_{L}(\mathbf{R})=\Psi_{\mathrm{T}}(\mathbf{R})^{-1} \mathcal{H} \Psi_{\mathrm{T}}(\mathbf{R})
$$

Since $\rho(\mathbf{R})$ is a positive quantity and integrates to 1 , we can interpret it as a probability distribution and use classical Monte Carlo techniques to sample a set of configurations $\left\{\mathbf{R}_{m}\right\}$ distributed according to $\rho(\mathbf{R})$. The expectation value can then be estimated as an average of the local energy $E_{L}(\mathbf{R})$ evaluated on these configurations:

$$
E_{V} \approx \frac{1}{M} \sum_{m=1}^{M} E_{L}\left(\mathbf{R}_{m}\right)
$$

Note that in this derivation, we can substitute the Hamiltonian $\mathcal{H}$ with any operator $\mathcal{O}$ diagonal in space representation.

For a realistic molecular system, the square of the many-body wave function is a complicated probability distribution in a high-dimensional space, whose normalization is usually not easy to compute. Therefore, we cannot use direct sampling techniques but we employ the classical Metropolis algorithm [31] to generate a sequence of configurations $\left\{\mathbf{R}_{m}\right\}$ distributed according to $\rho(\mathbf{R})$. The Metropolis algorithm is a general method to sample an arbitrary probability distribution without knowing its normalization. Here, we employ the VMC algorithm as described in Ref. 32, which uses a non-symmetrical transition matrix in the Metropolis algorithm, and which we properly modified to deal with pseudopotentials.

\section{Many-Body Wave Functions Used in Quantum Monte Carlo}

To understand the functional form of the wave function most commonly used in quantum Monte Carlo, let us recall that methods such as configuration interaction 
(CI) expand the wave function in a linear combination of Slater determinants of single-particle orbitals and are therefore plagued by a slow convergence, in part because of the difficulty in describing the cusps at the electron-electron coalescence points. When the electrons approach each other, the potential energy diverges at infinity, so the kinetic energy must have an opposite divergence to the potential to keep the local energy finite. This cancellation can be enforced if the trial wave function satisfies the so-called "cusp conditions" and displays a proper discontinuity of the derivatives at the coalescence points. By introducing an explicit dependence on the interelectronic distance, quantum Monte Carlo can exactly impose such conditions and, consequently, employ a much more compact representation of the wave function, which is usually given by a sum of few determinants (hundreds and not millions like in a CI calculation) multiplied by a component with the proper cusp behavior.

The trial wave functions most commonly used in quantum Monte Carlo calculations are of the so-called Jastrow-Slater form, namely, a product between a sum of determinants of single-particle orbitals, and a Jastrow correlation factor. In the spin-assigned form, a Jastrow-Slater wave function can be written as:

$$
\Psi\left(\mathbf{r}_{1}, \ldots, \mathbf{r}_{N}\right)=\mathcal{J}\left(\mathbf{r}_{1}, \ldots, \mathbf{r}_{N}\right) \sum_{k} d_{k} D_{k}^{\uparrow}\left(\mathbf{r}_{1}, \ldots, \mathbf{r}_{N_{\uparrow}}\right) D_{k}^{\downarrow}\left(\mathbf{r}_{N_{\uparrow}+1}, \ldots, \mathbf{r}_{N}\right),
$$

where $D_{k}^{\uparrow}$ and $D_{k}^{\downarrow}$ are Slater determinants of single particle orbitals for the up and down spin electrons, respectively. The orbitals are a linear combination of Gaussian basis functions when pseudopotentials are employed. The Jastrow factor is a positive function of the interparticle distances and is of particular important for the description of electronic correlation at short and intermediate interelectron distances. In particular, the electron-electron cusp conditions are imposed through the Jastrow factor.

The form of the Jastrow factor we use depends on electron-electron and the electron-nucleus distances and accounts for electron-electron, electron-nucleus and electron-electron-nucleus correlations:

$$
\begin{aligned}
\mathcal{J}\left(\mathbf{r}_{1}, \ldots, \mathbf{r}_{N}\right)=\prod_{\alpha, i} \exp & \left\{A\left(r_{i \alpha}\right)\right\} \times \prod_{i<j} \exp \left\{B\left(r_{i j}\right)\right\} \times \\
& \times \prod_{\alpha, i<j} \exp \left\{C\left(r_{i \alpha}, r_{j \alpha}, r_{i j}\right)\right\} .
\end{aligned}
$$

The electron-nucleus terms A must be included if the single-particle orbitals in the determinants are taken from a DFT or a HF calculation and not reoptimized in the presence of the Jastrow factor. This term corrects for the fact the electron-electron term reduces/increases the single-particle density in high/low density regions, and leads in general to a worse density than the original DFT or HF density. The electron-electron term B ensures that the electron-electron cusp conditions are satisfied, and keeps the electrons apart. Finally, the electron-electron-nucleus terms $\mathrm{C}$ can in principle exactly describe a two-electron atom or ion in an S state. Higher 
body correlations are clearly less important since it is rare for three or more electrons to be close since at least two electrons must necessarily have the same spin.

Since the Jastrow factor should be finite at large distances, we introduce scaled variables $\bar{r}=\left(1-e^{-\kappa r}\right) / \kappa$ for the $\mathrm{A}$ and $\mathrm{B}$ terms, and $\bar{r}=e^{-\kappa r}$ for the $\mathrm{C}$ terms. In this thesis, we use the following form:

$$
\begin{aligned}
A\left(r_{i \alpha}\right) & =\frac{a_{1} \bar{r}_{i \alpha}}{1+a_{2} \bar{r}_{i \alpha}}+\sum_{p=2}^{N_{\text {ord }}^{a}} a_{p+1} \bar{r}_{i \alpha}^{p} \\
B\left(r_{i j}\right) & =\frac{b_{1} \bar{r}_{i j}}{1+b_{2} \bar{r}_{i j}}+\sum_{p=2}^{N_{\text {ord }}^{b}} b_{p+1} \bar{r}_{i j}^{p} \\
C\left(r_{i \alpha}, r_{j \alpha}, r_{i j}\right) & =\sum_{p=2}^{N_{\text {ord }}^{c}} \sum_{0}^{k=p-1} \sum_{0}^{l=l_{\max }} c_{m k l} \bar{r}_{i j}^{k}\left(\bar{r}_{i \alpha}^{l}+\bar{r}_{j \alpha}^{l}\right)\left(\bar{r}_{i \alpha} \bar{r}_{j \alpha}\right)^{m},
\end{aligned}
$$

where $m=(p-k-l) / 2$, and $l_{\max }$ is $p-k$ if $k \neq 0$ and $p-k-2$ if $k=0$. Only terms for which $m=(p-k-l) / 2$ is an integer are included. The $a$ and $c$ coefficients are different for different atom types. The only spin dependence is in $b_{1}$ which is is used to satisfy the electron-electron cusp conditions: $b_{1}=1 / 2$ for antiparallel spin, and $b_{1}=1 / 4$ for parallel electrons.

The quality of the trial wave function affects the statistical efficiency of the QMC algorithms as well as the final accuracy of the results. The parameters in the Jastrow factor should therefore be optimized and it would be preferable if also the parameters in the determinantal component (CI and LCAO coefficients) were optimized since the optimal values will be different from the DFT or MCSCF ones, which are instead optimal in the absence of the Jastrow factor. For the optimization of the parameters in the trial wave function of a system in its ground state, we use the so-called linear optimization method within energy minimization [33]. Since we are also interested in excited states, we want to be able to optimize the parameters of the multiple (ground and excited) orthogonal states described by the wave functions:

$$
\Psi_{I}=\sum_{i=1}^{N_{\mathrm{CSF}}} c_{i}^{I} \mathcal{J} C_{i} .
$$

which share the same Jastrow factor and orbitals but different linear coefficients. To this end, we employ a generalization of the linear method to state-average (SA) optimization [34] to determine a set of orbitals and a Jastrow factor which give a comparably good description of the states under considerations while preserving orthogonality among the states.

\section{Diffusion Monte Carlo}

While VMC is a very useful to explore which type of correlation is important in the description of the system of interest, all results will of course depend on the 
quality of the input wave function. Moreover, there is not an unique and automatic way to construct this wave function, whose functional form must be tailored for each particular study. Projector Monte Carlo is a different flavor of QMC, which removes (at least in part) the bias of the trial wave function from the results. The idea of projection Monte Carlo is to use an operator that inverts the spectrum of $\mathcal{H}$ to project out the ground state of $\mathcal{H}$ from a given trial state.

Here, we only discuss the particular choice of operator in the diffusion Monte Carlo (DMC) approach, which we use in our calculations. In DMC, the projection operator is given by $\exp \left[-\tau\left(\mathcal{H}-E_{\mathrm{T}}\right)\right]$, and is applied to an initial trial wave function to obtain the sequence of wave functions:

$$
\Psi^{(n)}=e^{-\tau\left(\mathcal{H}-E_{\mathrm{T}}\right)} \Psi^{(n-1)} .
$$

If we expand the initial wave function $\Psi^{(0)}$ on the eigenstates $\Psi_{i}$ with energies $E_{i}$ of $\mathcal{H}$, we obtain for $\Psi^{(n)}$ :

$$
\Psi^{(n)}=\sum_{i} \Psi_{i}\left\langle\Psi^{(0)} \mid \Psi_{i}\right\rangle e^{-n \tau\left(E_{i}-E_{\mathrm{T}}\right)},
$$

Since the coefficients of the excited states die off exponentially fast relative to the one of the ground state, we obtain

$$
\lim _{n \rightarrow \infty} \Psi^{(n)}=\Psi_{0}\left\langle\Psi^{(0)} \mid \Psi_{0}\right\rangle e^{-n \tau\left(E_{0}-E_{\mathrm{T}}\right)} .
$$

Therefore, if we adjust the trial energy $E_{\mathrm{T}} \approx E_{0}$ to keep the over all normalization of $\Psi^{(n)}$ fixed, the projection gives us the ground state $\Psi_{0}$ of the Hamiltonian.

To understand how to proceed in practice, let us rewrite Eq. 2.40 in integral from as

$$
\Psi^{(n)}\left(\mathbf{R}^{\prime}, t+\tau\right)=\int \mathrm{d} \mathbf{R} G\left(\mathbf{R}^{\prime}, \mathbf{R}, \tau\right) \Psi^{(n-1)}(\mathbf{R}, t),
$$

where the Green's function is defined as

$$
G\left(\mathbf{R}^{\prime}, \mathbf{R}, \tau\right)=\left\langle\mathbf{R}^{\prime}\left|e^{-\tau\left(\mathcal{H}-E_{\mathrm{T}}\right)}\right| \mathbf{R}\right\rangle .
$$

This integral can be computed stochastically if we can sample the trial wave function and the Green's function in Eq. 2.43. Since, for fermions, the wave function is antisymmetric and cannot be interpret as a probability distribution, we will assume that, for the moment, we are dealing with bosons and return to this issue later.

For small $\tau$, the Green's function can be approximated via the Trotter-Suzuki formula as

$$
\left\langle\mathbf{R}^{\prime}\left|e^{-\mathcal{H} \tau}\right| \mathbf{R}\right\rangle \approx \frac{1}{(2 \pi \tau)^{3 N / 2}} \exp \left[-\frac{\left(\mathbf{R}^{\prime}-\mathbf{R}\right)^{2}}{2 \tau}\right] \exp [-\tau \mathcal{V}(\mathbf{R})] .
$$

Therefore, the iteration in Eq. 2.43 can be interpreted as a branching random walk: The first factor in the short-time Green's function is a diffusion step, while the second term multiplies the distribution by a positive scalar. Since the short-time expression of the Green's function is only valid in the limit of small $\tau$, the results of DMC calculations must be extrapolated for $\tau$ which goes to zero. 
The use of this Green's function leads to an unstable algorithm since the potential can vary significantly in configuration space or even be divergent as, for instance, the Coulomb potential when two particles approach each other. Even if the potential is bounded, the approach is inefficient with increasing system size since the potential is an extensive quantity and the branching factor therefore grows with the number of particles. It is possible to overcome these difficulties by using the so-called importance sampling algorithm [35], where we make use of the trial wave function $\Psi$ we have built within VMC. To do so, we start from Eq. 2.43, multiply each side by $\Psi$, and define the probability distribution $f^{(n)}(\mathbf{R})=\Psi(\mathbf{R}) \Psi^{(n)}(\mathbf{R})$ which satisfies

$$
f^{(n)}\left(\mathbf{R}^{\prime}, t+\tau\right)=\int \mathrm{d} \mathbf{R} \tilde{G}\left(\mathbf{R}^{\prime}, \mathbf{R}, \tau\right) f^{(n-1)}(\mathbf{R}, t) .
$$

The importance sampled Green's function is given by

$$
\tilde{G}\left(\mathbf{R}^{\prime}, \mathbf{R}, \tau\right)=\Psi\left(\mathbf{R}^{\prime}\right)\left\langle\mathbf{R}^{\prime}\left|e^{-\tau\left(\mathcal{H}-E_{\mathrm{T}}\right)}\right| \mathbf{R}\right\rangle / \Psi(\mathbf{R}) .
$$

For small $\tau$, the resulting drift-diffusion-branching short-time Green's function is given by

$$
\begin{array}{r}
\tilde{G}\left(\mathbf{R}^{\prime}, \mathbf{R}, \tau\right)=(2 \pi \tau)^{3 N / 2} \exp \left[-\frac{\left(\mathbf{R}^{\prime}-\mathbf{R}-\tau \mathbf{V}(\mathbf{R})\right)^{2}}{2 \tau}\right] \times \\
\times \exp \left\{-\tau\left[\left(E_{\mathrm{L}}(\mathbf{R})+E_{\mathrm{L}}\left(\mathbf{R}^{\prime}\right)\right) / 2-E_{\mathrm{T}}\right]\right\}+O\left(\tau^{2}\right) .
\end{array}
$$

where the quantum velocity is defined as

$$
\mathbf{V}(\mathbf{R})=\frac{\nabla \Psi(\mathbf{R})}{\Psi(\mathbf{R})}
$$

The use of importance sampling yields two improvements: i) The quantum velocity pushes the walkers to regions where the wave function is large; ii) the local energy $E_{\mathrm{L}}(\mathbf{R})$ instead of the potential $\mathcal{V}(\mathbf{R})$ appears in the branching factor. Since the local energy becomes a constant eigenvalue as the trial wave function approaches the exact eigenstate, we expect that a good trial wave function will lead to smaller fluctuations in the branching factor. Moreover, the instabilities for the Coulomb potential are removed since the wave function satisfies the cusp conditions.

So far, we have not addressed the issue that electrons are fermions and that the trial wave function must be antisymmetric, and therefore change sign. Algorithms which attempt to handle both signs of the wave function lead to the fermion sign problem, namely, that the bosonic component grows at the expenses of the fermionic one, and the antisymmetric signal is lost in the noise. To avoid this problem, we work in the so-called fixed-node approximation and solve the evolution equation with the boundary condition that the solution has the same nodes (the same zeros) as the given trial wave function. The Schrödinger equation is solved exactly inside the nodal regions but not at the nodes where the solution will have a discontinuity of the derivatives. The fixed-node solution is exact only if the nodes of the trial wave 
function are exact and, for approximate nodes, gives an upper bound to the exact energy.

DMC can also be used to study the excited states of a given Hamiltonian. If the state is the lowest state of a one-dimensional representation of the point group of the molecules, DMC will yield a solution which is variational. If the excited state is not the lowest state in its symmetry, we can use DMC in the fixed-node approximation and employ the nodes of a trial wave function, constructed to describe the desired excited state, to keep the solution from collapsing on the ground state. We will however not have a variational principle and we are only guaranteed that fixed-node DMC yields the exact solution if the nodes of the trial wave function are exact. Therefore, we may expect that the role of the trial wave function in DMC is even more important for excited states than for ground states since it not only imposes fermionic antisymmetry but also selects the state of interest.

\subsection{Quantum Mechanics in Molecular Mechan- ics Techniques}

In this thesis, we investigate the absorption in a complex biological systems comprising many thousands of atoms, for which a full quantum mechanical treatment is clearly impossible. Fortunately, the primary photoabsorption process often involves the chromophore and few surroundings residues, and is therefore spatially rather localized (the extent of this localization will be the subject of study in Chapter 6). Therefore, it is not unreasonable to attempt to partition the system into a smaller quantum subsystem and a larger environment to be simulated by less expensive classical molecular mechanics methods. These hybrid methods are called quantum mechanics in molecular mechanics (QM/MM) [36] approaches.

The classical MM calculations employ empirical force fields to describe the interactions of the atoms [37]. The chosen force field defines the functional form of the potential-energy function and the parameters set for the atoms. The functional form contains bonded terms, for example, bond stretching, bending of angles, and torsional angles. Furthermore, it contains non-bonded terms, which are van der Waals terms and the Coulomb interaction terms between the partial point charges of the atoms. For the interface between the MM and QM parts in the quantum Monte Carlo calculations, we adopt here the approach by Röthlisberger and coworkers [38] as implemented in the code CPMD [39]. The non-bonded interactions between the MM and the QM parts are modelled as

$$
H_{\mathrm{NB}}=\sum_{I \in M M} q_{I} \int \mathrm{d} \mathbf{r} \frac{\rho(\mathbf{r})}{\left|\mathbf{r}-\mathbf{r}_{I}\right|}+\sum_{I \in M M, J \in Q M} v_{\mathrm{vdW}}\left(r_{I J}\right)
$$

where $\rho(\mathbf{r})$ is the density of the electrons and the nuclei of the QM system, $q_{I}$ are the MM partial charges at positions $\mathbf{r}_{I}$, and the classical force field $v_{\mathrm{vdW}}$ regulates the van der Waals interactions between QM and MM atoms. 
If the QM system is close to some positively charged MM atoms, one encounters so-called spill-out effects of quantum charge towards the MM region, which are particularly severe when a plane-wave basis is used as in the CPMD code. To avoid these problems, the point charges in proximity of the QM system are screened so that the electrostatic interaction of electrons with the close MM atoms in the non-bonded Hamiltonian is

$$
H_{\mathrm{NB}}^{\mathrm{el}}=\sum_{I \in \mathrm{NN}} q_{I} \int \mathrm{d} \mathbf{r} \rho^{\mathrm{el}}(\mathbf{r}) v_{I}\left(\mathbf{r}-\mathbf{r}_{I}\right)
$$

where

$$
v_{I}(r)=\frac{r_{c I}^{n}-r^{n}}{r_{c I}^{n+1}-r^{n+1}}
$$

with $r_{c I}$ the covalent radius of the atom type $I$ and $\mathrm{n}=4$ [38]. Finally, we mention that the QM/MM boundary in our system cuts through a chemical bond of a molecule. In this case, we "cap" the broken bond with a hydrogen atom, which is seen by the QM system but not by the MM atoms.

\subsection{Computational Details}

We list here the codes employed in the various calculations. Computational details specific to each calculation will be given in each chapter.

The Gromacs [40] code is a set of computational tools to perform and analyze classical molecular dynamics simulations.

The CPMD [39] code is used to perform the QM/MM calculation within DFT as QM method, while the Gromos [41] classical force field code is used for the MM part. We employ the QM/MM interface developed by Röthlisberger and coworkers $[38,42]$. The CPMD code is a plane-wave/pseudopotential code particularly designed for ab-initio molecular dynamics.

The Molcas [43] code is a quantum chemistry code, based on Gaussian basis sets, that we use to perform CASSCF and CASPT2 calculations.

The Gaussian 09 [44] code is a quantum chemistry code we use to perform ground-state DFT and linear-response TDDFT calculations. It uses Gaussian basis sets, and a wide range of exchange-correlation functionals is available.

The Amsterdam Density Functional ADF [45] code is a software package for first-principles electronic structure DFT calculations, using Slater functions for the construction of the orbitals. We employ this code to perform sub-system DFT calculations.

The GAMESS [46] code is a ab initio quantum chemistry package which we mostly use to generate the starting QMC wave functions either through a DFT or a SA-CASSCF calculation. This code employs Gaussian basis sets. The electrostatic effect of the protein environment can be introduced through the use of screened point charges described by the potential

$$
v(r)=\frac{q}{r}\left(1-A e^{-B r}\right)
$$


where $q$ is the value of the charge, and A and B are free parameters. We choose $A=$ 1 to remove the divergence at the origin, and adjust the value of the $\mathrm{B}$ to reproduce the CPMD potential outside the maximum. We find that the choice $B=1 / r_{c}^{2}$ where $r_{c}$ is the CPMD core radius gives similar potentials in the valence region.

Among other codes employed are the Dalton [47] code for coupled-cluster calculations and the ORCA [48] code for NEVPT2 calculations. Both these codes employ gaussian basis set.

Finally, the code CHAMP is used for all the quantum Monte Carlo calculations. It can perform VMC and DMC calculations, and optimize the wave function parameters by energy minimization.

\subsection{Bibliography}

[1] C. J. Cramer, Essentials of Computational Chemistry, 2nd ed. (Wiley, 2004).

[2] F. Jensen, Introduction to Computational Chemistry, 2nd ed. (Wiley, 2007).

[3] D. Roca-Sanjuán, F. Aquilante, and R. Lindh, WIREs Comput Mol Sc 2, 585 (2012).

[4] C. Angeli, M. Pastore, and R. Cimiraglia, Theor. Chem. Acc. 117, 743 (2007).

[5] W. Koch and M. C. Holthausen, A Chemist's Guide to Density Functional Theory (Wiley-VCH, 2000).

[6] P. Hohenberg and W. Kohn, Phys. Rev. 136, B864 (1964).

[7] W. Kohn and L. J. Sham, Phys. Rev. 140, A1133 (1965).

[8] G. E. Scuseria and V. N. Staroverov, in Theory and Applications of Computational Chemistry - The First Forty Years, edited by C. E. Dykstra, G. Frenking, K. S. Kim, and G. E. Scuseria (Elsevier B.V, 2005), pp. 669-724.

[9] A. D. Becke, Phys. Rev. A 38, 3098 (1988).

[10] C. Lee, W. Yang, and R. G. Parr, Phys. Rev. B 37, 785 (1988).

[11] J. P. Perdew, K. Burke, and M. Ernzerhof, Phys. Rev. Lett. 77, 3865 (1996).

[12] A. D. Becke, J. Chem. Phys. 98, 5648 (1993).

[13] P. J. Stephens, F. J. Devlin, C. F. Chabalowski, and M. J. Frisch, J. Phys. Chem. 98, 11623 (1994).

[14] Y. Zhao and D. G. Truhlar, J. Chem. Phys. 125, 194101 (2006).

[15] Y. Zhao and D. G. Truhlar, J. Phys. Chem. A 110, 13126 (2006). 
[16] Y. Zhao and D. Truhlar, Theor. Chem. Acc. 120, 215 (2008).

[17] Y. Zhao and D. G. Truhlar, Acc. Chem. Res. 41, 157 (2008).

[18] K. A. Nguyen, P. N. Day, and R. Pachter, J. Chem. Phys. 135, 074109 (2011).

[19] H. Iikura, T. Tsuneda, T. Yanai, and K. Hirao, J. Chem. Phys. 115, 3540 (2001).

[20] T. Yanai, D. P. Tew, and N. C. Handy, Chem. Phys. Lett 393, 51 (2004).

[21] O. A. Vydrov, J. Heyd, A. V. Krukau, and G. E. Scuseria, J. Chem. Phys. 125, 074106 (2006).

[22] O. A. Vydrov and G. E. Scuseria, J. Chem. Phys. 125, 234109 (2006).

[23] M. E. Casida, J. Mol. Struct. 914, 3 (2009).

[24] E. Runge and E. Gross, Phys. Rev. Lett. 52, 997 (1984).

[25] R. van Leeuwen, Phys. Rev. Lett. 82, 3863 (1998).

[26] M. E. Casida, in Recent Advances in Density Functional Methods, Part I, edited by D. P. Chong (World Scientific, 1995), p. 155.

[27] D. J. Tozer, R. D. Amos, N. C. Handy, B. O. Roos, and L. S. Andres, J. Mol. Phys. 97, 859 (1999).

[28] M. E. Casida, F. Gutierrez, J. Guan, F. X. Gadea, and D. Salahub, J. Chem. Phys. 113, 7062 (2000).

[29] A. Dreuw, J. L. Weisman, and M. Head-Gordon, J. Chem. Phys. 119, 2943 (2003).

[30] A. Lüchow, WIREs Comput Mol Sc 1, 388 (2011).

[31] N. Metropolis, A. Rosenbluth, M. N. Rosenbluth, A. H. Teller, and E. Teller, J. Chem. Phys. 21, 1087 (1953).

[32] C. J. Umrigar, Phys. Rev. Lett. 71, 408 (1993).

[33] C. J. Umrigar, J. Toulouse, C. Filippi, S. Sorella, and R. G. Hennig, Phys. Rev. Lett. 98, 110201 (2007).

[34] C. Filippi, M. Zaccheddu, and F. Buda, J. Chem. Theory Comput. 5, 2074 (2009).

[35] D. M. Ceperley and B. J. Alder, Phys. Rev. Lett. 45, 566 (1980).

[36] H. M. Senn and W. Thiel, Angew. Chem. Int. Ed. 48, 1198 (2009).

[37] A. D. Mackerell, J. Comput. Chem. 25, 1584 (2004). 
[38] A. Laio, J. VandeVondele, and U. Rothlisberger, J. Chem. Phys. 116, 6941 (2002).

[39] CPMD, http://www.cpmd.org/, Copyright IBM Corp 1990-2008, Copyright MPI für Festkörperforschung Stuttgart 1997-2001.

[40] B. Hess, C. Kutzner, D. van der Spoel, and E. Lindahl, J. Chem. Theory Comput. 4, 435 (2008).

[41] W. F. van Gunsteren, S. R. Billeter, A. A. Eising, P. H. Hünenberger, P. Krüger, A. E. Mark, W. R. P. Scott, I. G. Tironi, Biomolecular Simulation: The GROMOS96 Manual and User Guide; Vdf Hochschulverlag AG an der ETH Zurich: Zurich, 1996.

[42] A. Laio, J. VandeVondele, and U. Rothlisberger, J. Phys. Chem. B 106, 7300 (2002).

[43] F. Aquilante, L. De Vico, N. Ferré, G. Ghigo, P.-A. Malmqvist, P. Neogrády, T. B. Pedersen, M. Pitoňák, M. Reiher, B. O. Roos, L. Serrano-Andrés, M. Urban, V. Veryazov, and R. Lindh, J. Comput. Chem. 31, 224 (2010).

[44] M. J. Frisch, G. W. Trucks, H. B. Schlegel, G. E. Scuseria, M. A. Robb, J. R. Cheeseman, G. Scalmani, V. Barone, B. Mennucci, G. A. Petersson, H. Nakatsuji, M. Caricato, X. Li, H. P. Hratchian, A. F. Izmaylov, J. Bloino, G. Zheng, J. L. Sonnenberg, M. Hada, M. Ehara, K. Toyota, R. Fukuda, J. Hasegawa, M. Ishida, T. Nakajima, Y. Honda, O. Kitao, H. Nakai, T. Vreven, J. A. Montgomery, Jr., J. E. Peralta, F. Ogliaro, M. Bearpark, J. J. Heyd, E. Brothers, K. N. Kudin, V. N. Staroverov, R. Kobayashi, J. Normand, K. Raghavachari, A. Rendell, J. C. Burant, S. S. Iyengar, J. Tomasi, M. Cossi, N. Rega, J. M. Millam, M. Klene, J. E. Knox, J. B. Cross, V. Bakken, C. Adamo, J. Jaramillo, R. Gomperts, R. E. Stratmann, O. Yazyev, A. J. Austin, R. Cammi, C. Pomelli, J. W. Ochterski, R. L. Martin, K. Morokuma, V. G. Zakrzewski, G. A. Voth, P. Salvador, J. J. Dannenberg, S. Dapprich, A. D. Daniels, Ö. Farkas, J. B. Foresman, J. V. Ortiz, J. Cioslowski, and D. J. Fox, Gaussian 09 Revision A.02, Gaussian Inc. Wallingford CT 2009.

[45] ADF2012, SCM, Theoretical Chemistry, Vrije Universiteit, Amsterdam, The Netherlands, http://www.scm.com.

[46] M. W. Schmidt, K. K. Baldridge, J. A. Boatz, S. T. Elbert, M. S. Gordon, J. H. Jensen, S. Koseki, N. Matsunaga, K. A. Nguyen, S. Su, T. L. Windus, M. Dupuis, and J. A. M. Jr, J. Comput. Chem. 14, 1347 (1993).

[47] DALTON, an ab initio electronic structure program, release 2.0, see http://www.kjemi.uio.no/software/dalton/dalton.html (2005). 
[48] Neese, F. ORCA - an ab initio, Density Functional and Semiempirical program package, Version 2.8. Max-Planck-Insitut für Bioanorganische Chemie, Mülheim an der Ruhr, 2011. 


\section{Chapter 3}

\section{Photoisomerization of the Retinal Chromophore in a New Light ${ }^{\dagger}$}

We present a systematic investigation of the structural relaxation in the excited state of model retinal chromophores in the gas phase using the complete-active-space selfconsistent-theory (CASSCF), multiconfigurational second-order perturbation theory (CASPT2), quantum Monte Carlo (QMC), and coupled cluster (CC) methods. In contrast to the CASSCF photoisomerization mechanism of bond inversion followed by torsion around formal double bonds, we find that the other approaches predict an initial skeletal relaxation which does not lead to bond inversion but to a rather flexible retinal chromophore with longer bonds and with the bond-length pattern of the ground state being partly preserved. The relaxation proceeds then preferentially via partial torsion around formal single bonds and does not reach a conical intersection region. Our findings are compatible with solution experiments which indicate the existence of multiple minima and relaxation pathways, some of which are nonreactive, do not lead to photoproducts via conical intersection, and are dominant in solution. Our results also demonstrate the importance of a balanced description of dynamical and static correlation in the excited-state gradients and raise serious concerns on the common use of the CASSCF method to investigate structural properties of photoexcited retinal systems.

\subsection{Introduction}

The absorption of visible light and its conversion to other forms of energy is at the heart of some of the most fundamental processes in biology. An important example of light absorption initiating a biological response is the primary event of vision [1] where light induces the cis-trans isomerization of the photosensitive 11-cis retinal chromophore in Rhodopsin [2] and other visual pigments, activating a cascade

\footnotetext{
${ }^{\dagger}$ This chapter has been published as O. Valsson and C. Filippi, "Photoisomerization of Model Retinal Chromophores: Insight from Quantum Monte Carlo and Multiconfigurational Perturbation Theory", J. Chem. Theory Comput. 2010, 6, 1275-1292
} 
of chemical reactions which ultimately culminate in the stimulation of the optical nerve [3]. The initial photoisomerization process is one of the fastest photochemical reactions in nature occurring within a few hundred femtoseconds [4] and the protein environment plays a central role in guiding the reaction. In solution, the dynamics of retinal is in fact quite different than in the protein, namely, about 20 times slower [5] and much less efficient [6,7]. Even though femtosecond spectroscopy studies have extensively investigated the primary isomerization step of retinal [8-14], the detailed nature of the molecular mechanism in the initial excited-state reaction and the exact role of the protein environment are still not understood [12].

Theoretically, a large number of calculations with a variety of quantum chemical methods have been performed to investigate the structural and spectroscopic properties as well as the nature of the photoisomerization mechanism of retinal and retinal models in the gas phase [15-41] and in the protein environment [24,42-61]. Given the large size of the retinal chromophore, most calculations including the protein via quantum mechanics/molecular mechanics (QM/MM) approaches have mainly focused on obtaining a realistic representation of the structural model in the ground state and understanding the environmental effects on the absorption properties. Interestingly, even though all investigations employing different techniques appear to reproduce the correct experimental absorption value, the reasons behind this agreement are contrasting and there are fundamental differences concerning the structure of the chromophore, the protonation of nearby residues, and the overall role of the protein in tuning the spectral properties $[46,52,53,57,60]$. If nailing down the exact role of the environment on the Franck-Condon excitation has proven elusive, the computation of dynamical properties of photoexcited retinal in the gas phase as well as in the protein is an even harder task since it requires a uniformly reliable computation of excited-state potential energy surfaces and the availability of analytical energy gradients for geometric optimization and dynamical runs.

To date, most excited-state geometrical investigations have employed the lowlevel complete-active-space self-consistent-field (CASSCF) approach for the relaxation of retinal in the gas phase $[15-18,33,34,38,46]$ with also few attempts to simulate the dynamics of retinal in the protein environment $[45,55,61]$. The excited-state energies on the CASSCF structures are often refined in single-point calculations with higher-level approaches such as multiconfigurational perturbation theory (CASPT2) to partially account for dynamical correlation largely missing in the CASSCF description. These studies have led to the generally accepted picture that photoisomerization begins with an in-plane skeletal relaxation which yields bond inversion and proceeds via a torsional motion around carbon-carbon bonds having double-bond character in the ground state $[16,18,34,38]$. The chromophore is then funneled into a conical intersection region which leads to the ground-state trans photoproduct. Recent calculations of Send and Sundholm based on coupled cluster (CC) theory have however challenged this picture as they obtain a rather different excited-state relaxation mechanism of retinal models in the gas phase $[29,31,35,37]$. The initial relaxation in the excited state at the $\mathrm{CC}$ level does not yield bond inversion but the lengthening of most bonds while preserving the general bond-length pattern of 
the ground state. The subsequent torsional motion is around carbon-carbon bonds holding single-bond character in the ground state. However, these CC calculations have been dismissed on the basis of being single reference and thus lacking a proper description of static correlation as compared to the CASSCF approach [36]. This response reflects the general acceptance of CASSCF as an adequate tool for the investigation of excited-state structural properties of retinal and other photosensitive chromophores.

In the present work, we perform a thorough investigation of the initial excitedstate relaxation from the Frank-Condon point of model retinal chromophores in the gas phase, and employ CASPT2 and quantum Monte Carlo (QMC) in addition to the CASSCF and CC methods. The CASPT2 approach is well established and is considered a benchmark method for the computation of excited-state properties but its use for retinal models has been mostly confined to single-point calculations, in-plane geometrical relaxation of few models [20], and constraint optimization of a minimal chromophore model [19,20,23,41]. The QMC method is instead less common in the field of theoretical photochemistry and its use for excited-state geometrical optimization is novel. QMC has recently given promising results in the study of various photoactive molecules [62-66] and a favorable comparison with CASPT2 will further establish its use for investigating photochemical problems. At the cost of being computationally more expensive, CASPT2 and QMC methods can give an accurate and balanced description of both static and dynamical correlation and therefore represent an ideal tool to clarify the nature of the microscopic mechanism in the photoisomerization of retinal and to resolve the disagreement between the generally accepted CASSCF picture and the recent, controversial CC results.

We find that our in-plane geometrical relaxations from the Frank-Condon point of retinal models show a consistent, good agreement among the CASPT2, CC, and QMC approaches, which give excited-state structures not characterized by bondlength inversion in striking contrast to the results obtained with the CASSCF approach. Photoexcitation therefore weakens all bonds, which stretch and become partly more similar in length while preserving the general bond-length pattern of the ground state. To investigate a non-trivial minimum energy path out of plane, we consider a model with four double bonds and find that the excited-state relaxation at the CASPT2 level proceeds preferentially via a partial torsional motion around a formal single bond and does not lead to a conical intersection region. To assess the existence of a reactive path at the CASPT2 level, we also study the constrained excited-state isomerization around a formal double bond for the same model. We find that the system encounters a small barrier to isomerization at rather large angles of rotation, beyond which it is funneled towards a conical intersection region characterized by bond inversion.

Therefore, in agreement with previous CC calculations, our results support the picture of a rather flexible retinal chromophore in the excited state as compared to the CASSCF excited chromophore which can only twist around formal double bonds. These findings are compatible with the observation in solution experiments of the existence of multiple minima, possibly corresponding to different torsional 
conformations, and multiple excited-state paths. Some of these paths are reactive and yield a photoproduct via a conical intersection while others are non-reactive, do not lead to a conical intersection, and are dominant in solution [67]. Finally, our results demonstrate the importance of including an accurate description of dynamical correlation also in the excited-state gradients and raise serious concerns about the common use of CASSCF in investigating excited-state structures of retinal systems.

In Section 3.2, we briefly present the methods used in this paper and focus on the description of the QMC geometrical optimization. In Section 3.3, we describe the computational details and, in Section 3.4, we introduce the model retinal chromophores we investigate. In Sections 3.5-3.7, we present the results for the vertical excitation energy, the in-plane geometrical relaxation, and the minimum energy path or out-of-plane geometrical relaxation in the excited state. Finally, in Section 3.8, we discuss our results and conclude.

\subsection{Methods}

In this work, we employ a wide range of ab initio quantum chemical methods. While we refer the reader to appropriate textbooks [68] for a discussion of the more traditional CASSCF, CASPT2, and CC approaches, we briefly review below the less common QMC methods [69]. In particular, we focus on the procedure we follow to perform geometrical optimization within variational Monte Carlo (VMC), which is non-standard, and on how we address stability issues in the calculation of energy gradients.

\subsubsection{QMC Methods}

QMC methods provide an accurate and balanced description of dynamical and static electronic correlation in both molecular and extended systems [69]. Their application to the description of the excited-state properties of photoactive molecules has already given promising results [62-66].

A crucial ingredient which determines the quality of a QMC calculation is the many-body trial wave function, which is here chosen of the so-called Jastrow-Slater type. Since we treat multiple states of the same symmetry, we write the groundand excited-state wave functions as linear combination of spin-adapted configuration state functions (CSF) multiplied with a Jastrow correlation factor,

$$
\psi_{I}=\mathcal{J} \sum_{i=1}^{N_{\mathrm{CSF}}} c_{i}^{I} C_{i}
$$

where different states depend on their individual linear coefficients, $c_{i}^{I}$, but share a common set of single-particle orbitals and Jastrow factor, $\mathcal{J}$. We use here a Jastrow factor which correlates pairs of electrons and each electron separately with a nucleus, and employ different Jastrow factors to describe the correlation with different atom 
types. Since the optimal orbitals and expansion coefficients in $\psi_{I}$ may differ from the values obtained for instance in a CASSCF calculation in the absence of the Jastrow factor, it is important to reoptimize them in the presence of the Jastrow factor.

The parameters of the trial wave functions are optimized in an efficient and simple approach in a state-average (SA) fashion as described in Ref. 66. In this scheme, we iteratively alternate between optimizing the linear coefficients in the CSF expansion and the non-linear (Jastrow and orbital) coefficients where the quantity minimized is the weighted averaged energy over the states under consideration:

$$
E_{\mathrm{SA}}=\sum_{I} w_{I} \frac{\left\langle\Psi_{I}|\mathcal{H}| \Psi_{I}\right\rangle}{\left\langle\Psi_{I} \mid \Psi_{I}\right\rangle}
$$

where the weights are fixed and $\sum_{I} w_{I}=1$. At convergence, the averaged energy $E_{S A}$ is stationary with respect to all parameter variations subject to the orthogonality constraint while the energies of the states are stationary with respect to variations of the linear coefficients but not of the orbital or Jastrow parameters. In this approach, the wave functions are kept orthogonal and a generalized variational theorem applies.

The set of optimal linear coefficients is obtained by solving a generalized eigenvalue problem where the Hamiltonian and the overlap matrix on the basis functions $\mathcal{J} C_{i}$ are estimated within VMC by sampling a guiding function $\Psi_{g}$ chosen to have significant overlap with all states of interest. The use of a non-symmetric estimator of the Hamiltonian matrix yields a strong zero-variance principle and renders the approach particularly efficient [70]. To optimize the non-linear parameters, we employ the linear optimization method first developed for ground states [71] and recently extended to the state-average optimization of multiple states [66]. In this scheme, the non-linear minimization problem is linearized by working in the basis of the derivatives of the wave function with respect to the non-linear parameters. In the case of multiple states, the elements of the weighted averaged Hamiltonian and overlap matrices are computed in a single VMC run by sampling a guiding wave function $\Psi_{g}$. Both when determining the linear and the non-linear parameters, the guiding wave function is here chosen equal to $\sqrt{\sum_{I}\left|\Psi_{I}\right|^{2}}$.

The optimal trial wave functions are then used in diffusion Monte Carlo (DMC), which gives the best energy within the fixed-node approximation, that is, the lowestenergy state with the same zeros (nodes) as the trial wave function.

\subsubsection{VMC Geometrical Optimization}

The VMC geometrical optimization is performed in Z-matrix coordinates where the energy gradients with respect to the nuclear coordinates are obtained using numerical differentiation and correlated sampling [72].

To determine the interatomic forces at a given reference geometry, we construct a set of secondary geometries corresponding to small forward and backwards displacements of 0.001 a.u. for the bond lengths and $0.01^{\circ}$ for the bond and dihedral 
angles. The gradient in Z-matrix coordinates is computed as

$$
\mathbf{g}_{\gamma}=\left[E\left(\mathbf{x}+\delta x_{\gamma}\right)-E\left(\mathbf{x}-\delta x_{\gamma}\right)\right] / 2 \delta x_{\gamma},
$$

where $E$ is the total energy and $\delta x_{\gamma}$ is a displacement in the internal coordinate $\gamma$ with respect to the reference coordinates $\mathrm{x}$. The diagonal component of the Hessian can be obtained in the same run at no extra cost as

$$
\mathbf{h}_{\gamma}^{\text {diag }}=\left[E\left(\mathbf{x}+\delta x_{\gamma}\right)-2 E(\mathbf{x})+E\left(\mathbf{x}-\delta x_{\gamma}\right)\right] / \delta x_{\gamma}^{2} .
$$

The geometry is updated employing an approximate version of the Newton-Raphson method as

$$
\mathbf{x}_{\gamma}^{\prime}=\mathbf{x}_{\gamma}-\mathbf{g}_{\gamma} / \mathbf{h}_{\gamma}^{\operatorname{diag}}
$$

where $\mathrm{x}^{\prime}$ denote the new coordinates in Z-matrix representation. To stabilize the procedure against numerical noise, we add a constant parameter of $5 \times 10^{-5}$ to all diagonal elements of the Hessian.

The use of correlated sampling allows us to efficiently determine relative energies for different geometries from a single reference Monte Carlo walk. The reference walk is obtained by sampling the wave function $\Psi$ corresponding to the coordinates $\mathrm{x}$ and Hamiltonian $\mathcal{H}$ while the secondary geometries are characterized by the corresponding quantities $\mathbf{x} \pm \delta x_{\gamma}, \Psi_{\gamma}$, and $\mathcal{H}_{\gamma}$. Given a reference primary wave function, the secondary wave function is here simply obtained by recentering $\Psi$ at the coordinates $\mathbf{x} \pm \delta x_{\gamma}$ without altering the wave function parameters. The electronic coordinates of the secondary walk are obtained by stretching the primary walk with the nuclear coordinates through a space-warp transformation as described in Ref. 72. In the present work, we use the function $F(r)=r^{-4}$ for the space-warp transformation.

In summary, the procedure for the geometrical optimization is the following: $i$ ) The determinantal component of the initial wave function is obtained in a CASSCF calculation; $i$ ) all wave function parameters are optimized in a VMC run (we discuss later the importance of optimizing the orbital parameters); iii) the energy gradients are obtained in a correlated sampling VMC calculation; $i v$ ) the geometry is updated as described above. We note that, with the exception of the first iteration, step $i$ ) can be skipped since step $\mathrm{ii}$ ) can be performed starting from the wave function optimized at the previous geometry and recentered at the current geometry. This procedure is iterated until the bond length and bond angle gradients are of the order of 0.001 Hartree/Bohr and $0.0001 \mathrm{Hartree}^{\circ}$, respectively, that is, comparable to their error bars. Since the stochastic nature of VMC does not allow to assign one particular geometry as the minimum one, we perform 5-10 additional iterations after convergence and average the internal coordinates over these additional steps.

To decrease the computational effort, we keep fixed the carbon-hydrogen and nitrogen-hydrogen bond lengths and all the bond angles involving terminal hydrogen atoms. All other internal degrees of freedom are allowed to vary. 


\subsubsection{Stability of VMC Energy Gradients}

The computation of gradients in VMC poses some stability issues which we analyze by considering for simplicity the gradient expression without the use of the space-warp transformation. Then, the energy difference between the primary and a secondary geometry can be written as

$$
\begin{aligned}
& E(\mathbf{x})-E\left(\mathbf{x}+\delta x_{\gamma}\right)= \\
& \left\langle\frac{\mathcal{H} \Psi(\mathbf{R})}{\Psi(\mathbf{R})}-\frac{\mathcal{H}_{\gamma} \Psi_{\gamma}(\mathbf{R})}{\Psi_{\gamma}(\mathbf{R})} W_{\gamma}(\mathbf{R})\right\rangle_{\Psi^{2}},
\end{aligned}
$$

where $\langle\cdot\rangle$ denotes the statistical average over the configurations sampled in VMC from the distribution $\Psi^{2}$, and the weights are defined as

$$
W_{\gamma}(\mathbf{R})=\frac{\Psi_{\gamma}^{2}(\mathbf{R}) / \Psi^{2}(\mathbf{R})}{\left\langle\Psi_{\gamma}^{2}(\mathbf{R}) / \Psi^{2}(\mathbf{R})\right\rangle_{\Psi^{2}}} .
$$

If we expand this energy difference to linear order in $\delta x_{\gamma}$, we obtain a term proportional to

$$
\left\langle\frac{\mathcal{H} \Psi(\mathbf{R})}{\Psi(\mathbf{R})} \frac{\partial \log \Psi(\mathbf{R})}{\partial x_{\gamma}}\right\rangle_{\Psi^{2}} .
$$

Since the product inside the square brackets diverges as $1 / d^{2}$ when the distance $d$ from the nodes of $\Psi$ approaches zero, the estimator of eq 3.6 obtained by sampling the square of the primary wave function has infinite variance and it is not possible to obtain a stable energy difference. To cure this problem, we follow Ref. 73 and sample a different distribution which is non-zero at the nodes and is defined here as

$$
\Psi_{g}(\mathbf{R})=\Psi(\mathbf{R}) \frac{\max \left[\varepsilon, d_{n}(\mathbf{R})\right]}{d_{n}(\mathbf{R})}
$$

where $d_{n}(\mathbf{R})$ is a measure of the distance from the nodes,

$$
d_{n}(\mathbf{R})=\frac{1}{|\nabla \Psi(\mathbf{R}) / \Psi(\mathbf{R})|}
$$

and $\varepsilon$ is a cutoff parameter [74] chosen as $10^{-2}$. The average of Eq. 3.8 can then be rewritten as

$$
\left\langle\frac{\Psi^{2}(\mathbf{R})}{\Psi_{g}^{2}(\mathbf{R})} \frac{\mathcal{H} \Psi(\mathbf{R})}{\Psi(\mathbf{R})} \frac{\partial \log \Psi(\mathbf{R})}{\partial x_{\gamma}}\right\rangle_{\Psi_{g}^{2}}
$$

where the reweighting factor $\Psi^{2}(\mathbf{R}) / \Psi_{g}^{2}(\mathbf{R})$ removes the divergence of the products inside the brackets. This cures the problem of the infinite variance and allows us to obtain stable energy differences. 


\subsection{Computational Details}

We use the program MOLCAS 7.2 [75] to optimize the ground-state geometries of the model chromophores within all-electron MP2 and DFT with the B3LYP [76] functional. For the ground-state optimizations of the full retinal model (see Figure 3.1E), we employ the Gaussian 03 code [77]. The default convergence criteria are used for both codes.

We also use MOLCAS 7.2 for the all-electron CASSCF, CASPT2, and multistate (MS) CASPT2 [78] calculations. The state-average (SA) CASSCF calculations are performed with equal weights over the states of interest, and the two lowest states are used in the SA-CASSCF and MS-CASPT2 calculations. In the CASPT2 calculations, we employ the default IPEA zero-order Hamiltonian [79] unless otherwise stated, and indicate if an additional constant level shift [80] is added to the Hamiltonian. In the CASPT2 calculations for the complete 11-cis retinal chromophore, we use the Cholesky decomposition of the two-electron integrals [81] with the default threshold of $10^{-4}$. Analytical CASSCF and numerical CASPT2 gradients are used for geometrical optimizations and minimum energy path (MEP) calculations. In the CASPT2 calculations, we do not correlate as many lowest orbitals of $\sigma$ character as the number of heavy atoms in the model. The default convergence criteria are used for all calculations.

We perform the EOM-CC calculations with the ACESII [82] and CFOUR [83] codes. The $\mathrm{CC}$ calculations include approximate single and double excitations (CC2) and single and double excitations (CCSD). Default convergence criteria are used for all calculations and we do not correlate as many lowest orbitals of $\sigma$ character as the number of heavy atoms in the model.

The program package CHAMP [84] is used for the QMC calculations. We employ scalar-relativistic energy-consistent Hartree-Fock pseudopotentials [85] where the carbon, nitrogen, and oxygen $1 s$ electrons are replaced by a non-singular $s$ non-local pseudopotential and the hydrogen potential is softened by removing the Coulomb divergence. Different Jastrow factors are used to describe the correlation with different atom types and, for each atom type, the Jastrow factor consists of an exponential of the sum of two fifth-order polynomials of the electron-nuclear and the electron-electron distances, respectively [86]. We also test the effect of including an electron-electron-nuclear term. The starting determinantal components are obtained in CASSCF calculations which are performed with the program GAMESS(US) [87]. In all SA-CASSCF calculations, equal weights over the states are employed and the final CAS expansions are expressed on the weighted-average CASSCF natural orbitals. The CAS wave functions of the ground and excited states may be truncated with an appropriate threshold on the CSF coefficients and the union set of surviving CSF's for the states of interest are retained in the QMC calculations. The Jastrow correlation factor and the CI coefficients are optimized by energy minimization in a state-averaged sense within VMC with equal weights. When indicated in the text, also the orbitals are optimized along with the Jastrow and CI parameters. The pseudopotentials are treated beyond the locality approximation [88] and an imaginary 
time step of 0.05 or 0.075 a.u. is used in the DMC calculations.

In the DFT, CASSCF, CASPT2, and CC calculations, we employ either the correlation consistent (cc-pVxZ) [89] or the atomic natural orbital (ANO-L-VxZP) [90] basis sets. We use the ANO contraction schemes as defined in MOLCAS, that is, [3s $2 p 1 d] /[2 s 1 p]$ for ANO-L-VDZP, [4s3p2d1f]/[3s2p1d] for ANO-L-VTZP, and [5s4p3d2f]/[4s3p2d] for ANO-L-VQZP. In the single-point energy calculations, the ANO-L-VDZP basis set is generally used while the default basis in the geometrical optimization and MEP calculations is the cc-pVDZ. In the QMC calculations, we use the Gaussian basis sets [85] specifically constructed for our pseudopotentials. In particular, we employ the cc-pVDZ basis (denoted by D) and the D basis augmented with $\mathrm{s}$ and $\mathrm{p}$ diffuse functions [91] on the heavy atoms (denoted by $\mathrm{D}+$ ). We also use the $\mathrm{T}^{\prime}$ and $\mathrm{Q}^{\prime}$ basis sets which consist of the cc-pVTZ and cc-pVQZ, respectively, combined with the cc-pVDZ for hydrogen. The $g$ functions are not included in the $\mathrm{Q}^{\prime}$ basis. Most single-point energy calculations use the $\mathrm{D}+$ basis while geometrical optimizations employ the $\mathrm{D}$ basis.

\subsection{Retinal Models}

The 11-cis retinal chromophore consists of a conjugated carbon chain with a protonated Schiff base (PSB) at one end and a twisted $\beta$-ionone ring at the other end (see Figure 3.1E). It sits inside the protein pocket of rhodopsin, a seven helix transmembrane, where it is covalently bound to Lys-296 via the protonated Schiff base linkage. In theoretical gas phase studies, there has been no consistent choice of how to terminate the covalent bond between the positively charge nitrogen in the protonated Schiff base and Lys-296. A single hydrogen, a methyl but also a $n$-butyl group have often been used as termination, and this particular choice appears to influence only slightly the excitation energy [27,40]. Due to the large size of the 11-cis retinal chromophore, smaller protonated Shiff base models have been mainly investigated theoretically, which differ in the length of the conjugated chain and the absence of methyl groups with respect to the complete chromophore.

The retinal models studied in this work are shown in Figure 3.1 and range from the minimal model (Figure 3.1A) to the full 11-cis retinal chromophore (Figure 3.1E). The atom labeling shown for the 11-cis chromophore is adopted also for the other models so that the cis-to-trans isomerization bond is always between the $\mathrm{C}_{11}$ and $\mathrm{C}_{12}$ atoms, with atom numbering increasing from the carbon to the nitrogen end. For the models without the $\beta$-ionone ring, we introduce the naming convention $\operatorname{PSBx}(\mathrm{y})$ where $\mathrm{x}$ and $\mathrm{y}$ are the number of double bonds and methyl groups, respectively. The PSB3(0) $\left(\mathrm{C}_{5} \mathrm{H}_{6} \mathrm{NH}_{2}^{+}\right)$model (A) is the minimal model of the retinal chromophore and has already been extensively studied in the literature $[15,17,20,22,25,41]$. Since the methyl group at position $C_{13}$ plays an important role in accelerating the isomerization process $[15,17]$, we also consider the PSB3(1) $\left(\mathrm{C}_{6} \mathrm{H}_{8} \mathrm{NH}_{2}^{+}\right)$model (B), that is, the minimal model (A) with an added methyl group. The PSB4(1) $\left(\mathrm{C}_{8} \mathrm{H}_{10} \mathrm{NH}_{2}^{+}\right)$model (C) has one additional double bond 

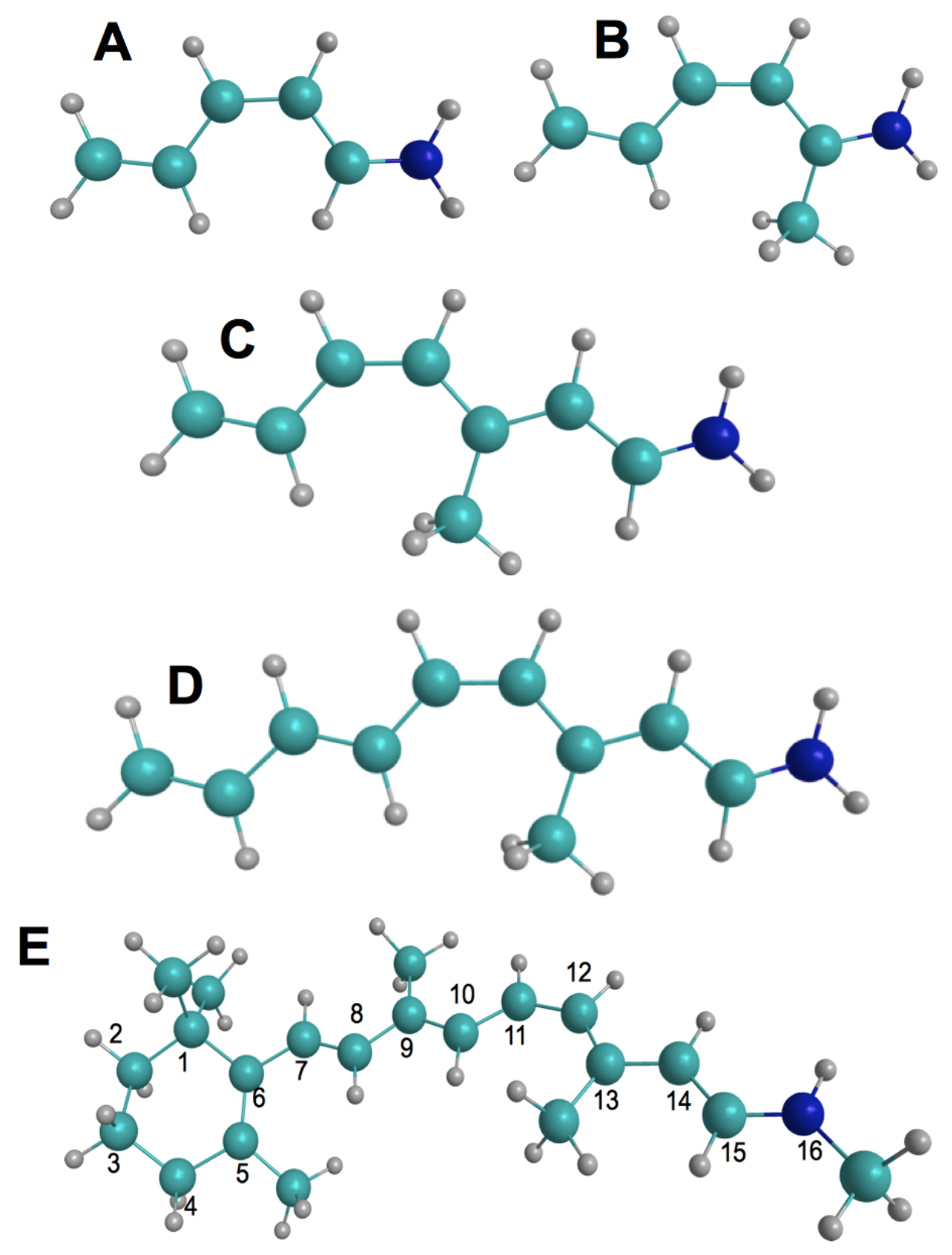

Figure 3.1: Model retinal chromophores. The atom numbering for chromophore (E) is used for all models, so the cis bond is always between $\mathrm{C}_{11}$ and $\mathrm{C}_{12}$. Cyan, blue and grey represent carbon, nitrogen, and hydrogen, respectively. 
and has been previously studied without the methyl group using the CC and TDDFT methods [29]. The PSB5(1) $\left(\mathrm{C}_{10} \mathrm{H}_{12} \mathrm{NH}_{2}^{+}\right)$model (D) has the full conjugated chain but is missing the $\beta$-ionone ring, and the complete 11-cis retinal chromophore (E) is here terminated with a single methyl group. With the exception of the 11-cis (E) chromophore, all other models are planar in the ground state. We note that a direct comparison with experiments is only possible for the vertical excitation energy of the 11-cis chromophore (E) since, to the best of our knowledge, none of the smaller models has been studied experimentally.

\subsection{Vertical Excitation Energies}

We compute the vertical excitation energies of the lowest singlet excited state $\left(\mathrm{S}_{1}\right)$ of all retinal models using the CASPT2, CC2, CCSD, VMC, and DMC approaches. The ground-state DFT/B3LYP geometries optimized with the cc-pVDZ basis are used. The model (E) is optimized with no symmetry constraint $\left(\mathrm{C}_{1}\right)$ while the other models are planar and are optimized in either $\mathrm{C}_{s}$ or $\mathrm{C}_{1}$ symmetry. The CASPT2 excitations are computed with the standard IPEA Hamiltonian (S-IPEA) and with the IPEA shift set to zero (0-IPEA), which was default prior to MOLCAS 6.4, in order to be compatible with previous calculations in the literature.

\subsubsection{Dependence on Basis Set and Other Parameters}

Before comparing the relative performance of the different methods, we focus on the minimal model (A) and investigate the dependence of the excitations on the choice of the basis set and other parameters which may affect the calculations. We begin with the MS-CASPT2 approach and show in Figure 3.2 the vertical excitations obtained with the double (D), triple $(\mathrm{T})$, and quadruple $(\mathrm{Q})$ zeta basis sets from the cc-pVxZ and ANO-L-VxZP series. We correlate all $6 \pi$ electrons in the reference configuration and use a different number $m$ of virtual $\pi$ orbitals in the CAS $(6, m)$ expansion. We note that single-state and MS-CASPT2 yield equivalent excitations for model (A).

We observe that the ANO-L-VxZP series gives a faster convergence for the CASPT2 excitation energy than the correlated consistent basis. The excitations computed with the $\mathrm{D}$ basis are only $0.05 \mathrm{eV}$ higher than the values obtained with the $\mathrm{T}$ and $\mathrm{Q}$ basis sets. On the other hand, in the cc-pVxZ series, the D excitations are 0.12 $\mathrm{eV}$ higher than the $\mathrm{T}$ values, which still differ from the $\mathrm{Q}$ results by $0.04 \mathrm{eV}$. The behavior of the CC2 and CCSD excitations with basis set is not shown in the Figure but parallels closely the one observed for the CASPT2 excitations. Therefore, since the ANO-L-VDZP basis set gives a good balance between accuracy and computational cost, it is used hereafter for all single-point CASPT2, CC2, and CCSD calculations.

We find that the CASPT2 results depend very strongly on the choice of the zeroorder Hamiltonian. The difference between the excitation energies obtained with the standard IPEA Hamiltonian and the IPEA shift set to zero is independent of the basis 


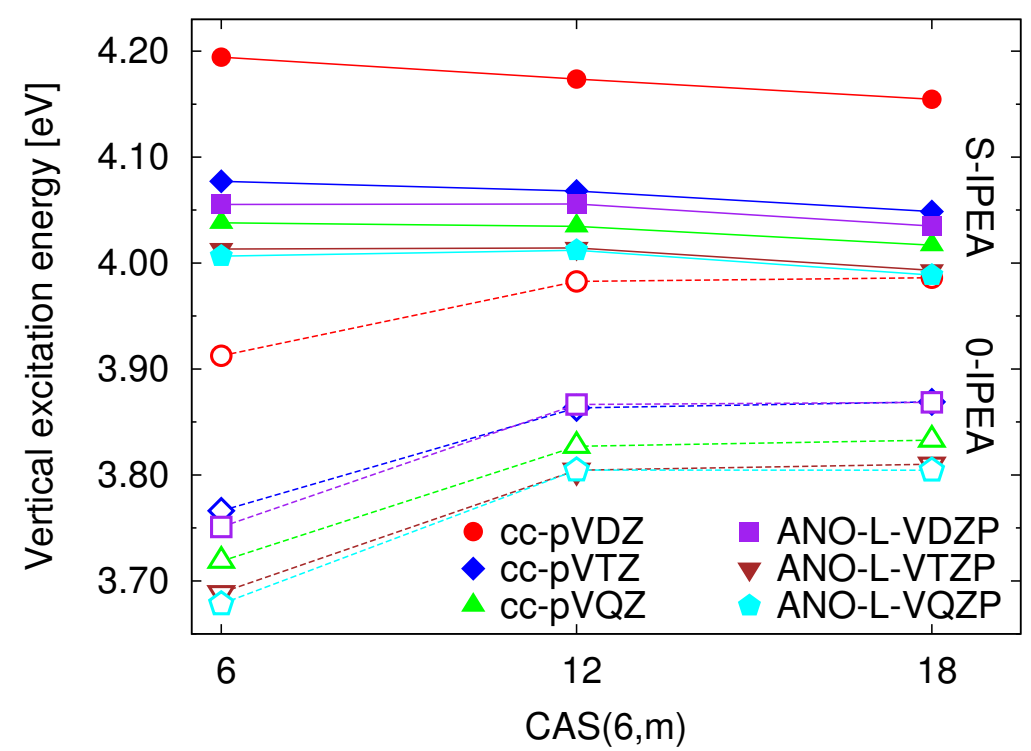

Figure 3.2: MS-CASPT2 vertical excitation $\left(\mathrm{S}_{1}\right)$ energies of the PSB3(0) model (A) computed with the standard IPEA Hamiltonian (S-IPEA, filled symbols) and with the IPEA shift set to zero (0-IPEA, empty symbols). The excitations are obtained with different basis sets and expansions CAS $(6, m)$ of 6 electrons in $m$ active orbitals. The ground-state DFT/B3LYP geometry is used.

set used and equal to about $0.3 \mathrm{eV}$ when a $\mathrm{CAS}(6,6)$ is employed. As expected, the dependence on the particular zero-order Hamiltonian is reduced as the wave function is improved, and the difference between the excitations with and without the IPEA shift becomes $0.2 \mathrm{eV}$ if the number of active $\pi$ orbitals in the CAS is increased from 6 to 18. Finally, we observe that the vertical energies obtained with the IPEA shift set to zero are much more sensitive to the dimension of the CAS since they increase by $0.07-0.12 \mathrm{eV}$ when $m$ goes from 6 to 18 , while the energies obtained with the standard IPEA Hamiltonian are quite stable and only decrease by about $0.02-0.04$ $\mathrm{eV}$.

In Table 3.1, we present an extensive QMC investigation for the minimal model (A) to assess how different ingredients in the trial wave function affect the excitation energy. The reference wave function is constructed from a $\mathrm{CAS}(6,6)$ expansion expressed on the weighted-averaged CASSCF natural orbitals in the D+ basis and truncated with a threshold of 0.02, where only the two-body Jastrow factor and CI coefficients are optimized in energy minimization in a SA fashion. Starting from this wave function, we investigate the effect of $i$ ) changing the threshold on the CAS $(6,6)$ expansion in the range $0.01-0.08 ; i i)$ increasing the number of active $\pi$ orbitals from 6 to 18 in the $\operatorname{CAS}(6, m)$ expansion; $i i i)$ including an electron-electronnuclear (e-e-n) term in the Jastrow factor in addition to the electron-nucleus (e-n) and electron-electron (e-e) components; $i v)$ optimizing the orbitals in a CAS $(6,6)$ wave 
Table 3.1: VMC and DMC vertical excitation $\left(\mathrm{S}_{1}\right)$ energies $(\mathrm{eV})$ of the PSB3(0) model (A), computed with different basis sets and CAS expansions expressed on the weighted-averaged natural orbitals. The $\operatorname{CAS}(6, m)$ active space includes all $6 \pi$ electrons occupied in the reference configuration and $m$ active $\pi$ orbitals. The threshold on the expansion and the corresponding number of determinants and CSFs are also listed. Unless indicated, only the Jastrow and CI parameters are optimized. The ground-state DFT/B3LYP geometry is used.

\begin{tabular}{llllllc} 
CAS $(6, m)$ & Thr. & Det./CSF & Jastrow & Basis & VMC & DMC \\
\hline$(6,6)$ & 0.01 & $183 / 79$ & e-n,e-e & D+ & $4.32(1)$ & $4.22(2)$ \\
$(6,6)$ & 0.02 & $101 / 47$ & e-n,e-e & D+ & $4.31(1)$ & $4.20(2)$ \\
$(6,6)$ & 0.04 & $66 / 31$ & e-n,e-e & D+ & $4.31(1)$ & $4.21(2)$ \\
$(6,6)$ & 0.08 & $23 / 10$ & e-n,e-e & D+ & $4.24(2)$ & $4.19(2)$ \\
$(6,6)^{a}$ & 0.08 & $23 / 10$ & e-n,e-e & D+ & $4.25(2)$ & $4.21(2)$ \\
$(6,6)^{b}$ & 0.08 & $23 / 10$ & e-n,e-e & D+ & $4.28(1)$ & $4.16(2)$ \\
$(6,6)$ & 0.02 & $103 / 48$ & e-n,e-e & D & $4.38(1)$ & $4.29(2)$ \\
$(6,6)$ & 0.02 & $103 / 48$ & e-n,e-e & $\mathrm{T}^{\prime}$ & $4.34(1)$ & $4.25(2)$ \\
$(6,6)$ & 0.02 & $103 / 48$ & e-n,e-e & $\mathrm{Q}^{\prime}$ & $4.34(1)$ & $4.22(2)$ \\
$(6,12)$ & 0.02 & $152 / 66$ & e-n,e-e & D+ & $4.29(1)$ & $4.22(2)$ \\
$(6,18)$ & 0.02 & $156 / 67$ & e-n,e-e & D+ & $4.29(1)$ & $4.22(2)$ \\
$(6,6)$ & 0.02 & $101 / 47$ & e-n,e-e,e-e-n & D+ & $4.32(2)$ & $4.24(2)$
\end{tabular}

${ }^{a}$ Orbitals optimized including 40 external orbitals;

${ }^{b}$ Orbitals optimized including 80 external orbitals.

function with a threshold of 0.08 both with 40 and 80 external orbitals included in the optimization; $v)$ using different basis sets $\left(\mathrm{D}, \mathrm{T}^{\prime}\right.$, and $\left.\mathrm{Q}^{\prime}\right)$. We find that the choice of basis has a significant effect on the QMC results as the VMC and DMC excitations computed with the $\mathrm{D}$ basis are higher by 0.06(2) and 0.09(3) $\mathrm{eV}$ than the corresponding $\mathrm{D}+$ values. Since the use of the larger $\mathrm{T}^{\prime}$ and $\mathrm{Q}^{\prime}$ basis sets brings the excitations in closer agreement with the D+ results, we employ below the D+ basis set to compute the QMC excitations of all model chromophores. For this choice of basis, other ingredients in the trial wave function appear to have a smaller effect on the VMC and DMC excitation energies which range between 4.24(2)4.32(2) and 4.16(2)-4.24(2) eV, respectively.

\subsubsection{Results}

We collect the vertical excitations of all retinal models computed using the MSCASPT2, CC2, CCSD, VMC, and DMC methods on the ground-state DFT/B3LYP geometries in Table 3.2. The VMC and DMC excitations are obtained using wave functions where the Jastrow and CI parameters are optimized by energy minimization in a SA fashion and the threshold on the CSF expansion is 0.08 for the (E) 
model and 0.02 for all other models. It is evident that, for all models, the CASPT2 excitations obtained with the IPEA shift set to zero are at variance and significantly lower than the results obtained with all other theoretical methods. The use of the standard IPEA Hamiltonian raises the excitation energies of all models by as much as $0.3 \mathrm{eV}$ and brings the CASPT2 values in close agreement with the CC2 results. The CCSD method yields excitations slightly higher by $0.11-0.17 \mathrm{eV}$ than the CC2 and CASPT2 results obtained with the IPEA Hamiltonian. Finally, the VMC excitations are always higher by $0.1-0.2 \mathrm{eV}$ than the DMC values which agree closely with the CCSD results.

Table 3.2: Vertical excitation $\left(\mathrm{S}_{1}\right)$ energies $(\mathrm{eV})$ of the retinal models. The MSCASPT2 energies are computed both with the standard IPEA Hamiltonian (S-IPEA) and without the IPEA shift (0-IPEA). The CAS $(n, n)$ expansion in the CASPT2 and QMC calculations includes all $\pi$ electrons in the reference configuration and an equal number $n$ of $\pi$ orbitals. CASPT2 and CC employ the ANO-L-VDZP basis, and QMC the D+ basis. The ground-state DFT/B3LYP geometries are used.

\begin{tabular}{lccccccc}
\hline Model & \multicolumn{3}{c}{ MS-CASPT2 } & CC2 & CCSD & VMC & DMC \\
\cline { 2 - 4 } & $n$ & 0-IPEA & S-IPEA & & & & \\
\hline & & & & & & & \\
A) PSB3(0) & 6 & 3.75 & 4.06 & 4.12 & 4.23 & $4.31(1)$ & $4.20(2)$ \\
B) PSB3(1) & 6 & 3.86 & 4.18 & 4.20 & 4.37 & $4.52(2)$ & $4.42(2)$ \\
C) PSB4(1) & 8 & 3.04 & 3.35 & 3.33 & 3.47 & $3.59(2)$ & $3.47(2)$ \\
D) PSB5(1) & 10 & 2.58 & 2.87 & 2.82 & 2.95 & $3.08(2)$ & $3.00(3)$ \\
E) 11-cis & 12 & $2.03^{a}$ & 2.30 & - & - & $2.59(3)$ & $2.41(3)$
\end{tabular}

${ }^{a}$ Constant level shift of 0.2 a.u.

For a comparison with experiments and previous theoretical work, we focus on the full 11-cis retinal chromophore (E) and collect the relevant results in Table 3.3. In line with previous calculations [24, 30, 58], we find that the excitation energy of the retinal chromophore depends strongly on the method used to determine its ground-state structure. The sequence of BLYP, B3LYP, MP2, and CASSCF geometries corresponds to an increase of the degree of bond-length alternation and of the twisting of the $\beta$-ionone ring from $-30^{\circ}$ to $-60^{\circ}$ (see Figure 3.3). Stronger bond alternation and larger twisting angles correspond to larger excitations energies and we find indeed an increase of $0.5 \mathrm{eV}$ in the CASPT2 excitation both with and without the IPEA shift, when going from the BLYP to the CASSCF geometry. A comparison with CASPT2 geometries of planar retinal models indicate that DFT and MP2 ground-state structures represent a better model for the retinal chromophore in the gas phase as shown in the Figure 3.6(a) and Figure SI-5 [92] and already observed in Ref. 20. Even though discarding the CASSCF structures significantly reduces the spread in excitations, we still have an uncertainty of about $0.1 \mathrm{eV}$ related to the choice of the particular DFT or MP2 geometry. 


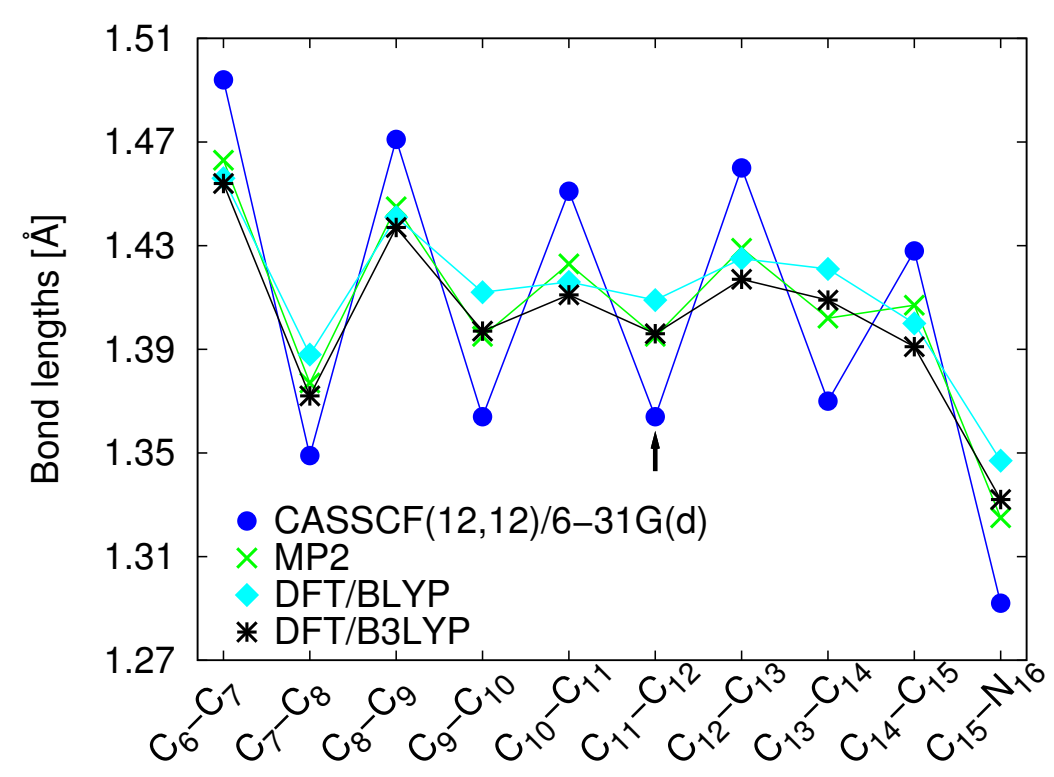

Figure 3.3: Ground-state bond lengths $(\AA)$ of the 11-cis chromophore (E) optimized using MP2, DFT/BLYP, and DFT/B3LYP and the cc-pVDZ basis. The CASSCF(12,12)/6-31G(d) geometry is from Ref. 27. The $\mathrm{C}_{5}-\mathrm{C}_{6}-\mathrm{C}_{7}-\mathrm{C}_{8}$ dihedral angles are $-29.7^{\circ},-33.5^{\circ},-40.5^{\circ}$, and $-68.8^{\circ}$ in BLYP, B3LYP, MP2, and CASSCF, respectively.

In Table 3.3, we present the single-state (SS) excitations in addition to results obtained with the MS-CASPT2 approach as done so far in this Section. As already mentioned, SS-CASPT2 and MS-CASPT2 give equivalent excitations within 0.01 $\mathrm{eV}$ for the smaller model (A) as it is expected given the large gap of about $4 \mathrm{eV}$ between the ground and excited states. However, as the size of the retinal model increases and the excitation decreases, SS-CASPT2 and MS-CASPT2 start to differ and this discrepancy grows faster when no IPEA shift is employed. For the 11-cis model (E) and a gap of about $2 \mathrm{eV}$, the difference amounts to about 0.10 and 0.15 $\mathrm{eV}$ with and without the IPEA shift, respectively, and is independent on the groundstate geometry. Therefore, the choice of performing single- or multi-state calculations within CASPT2 represents another internal parameter of the theory which affects the CASPT2 excitation in addition to the IPEA shift. We remark that, while MS-CASPT2 gives results which nicely parallel the DMC and CC excitations for all models, the difference between CASPT2 and other theories increases with system size if the single-state approach is used. The choice of the MS theory is our preference also for compatibility with the CASPT2 excited-state geometrical optimizations presented in the next Sections, where we employ the MS approach as it is not known a priori whether the molecule will encounter a conical intersection region during relaxation. 
Table 3.3: Single-state (SS) and MS-CASPT2 vertical excitation $\left(\mathrm{S}_{1}\right)$ energies $(\mathrm{eV})$ of the 11-cis retinal (E) chromophore. The DMC and experimental estimates are also listed. The geometries are optimized with the cc-pVDZ basis, and the CASPT2 calculations employ a CAS $(12,12)$ expansion and the ANO-L-VDZP basis.

\begin{tabular}{llcc}
\hline Method & Geometry & \multicolumn{2}{c}{$\mathrm{E}_{\text {exc }}$} \\
\hline SS-CASPT2 & & 0-IPEA & S-IPEA \\
& DFT/BLYP & $1.81^{b}$ & 2.12 \\
& DFT/B3LYP & $1.89^{b}$ & 2.20 \\
& MP2 & $1.92^{b}$ & 2.24 \\
& CASSCF $^{a}$ & $2.30^{b}$ & $2.65^{b}$ \\
MS-CASPT2 & & & \\
& DFT/BLYP & $1.96^{b}$ & 2.22 \\
& DFT/B3LYP & $2.03^{b}$ & 2.30 \\
& MP2 & $2.08^{b}$ & 2.35 \\
DMC/D+ & CASSCF & $2.42^{b}$ & $2.72^{b}$ \\
& DFT/BLYP & $2.32(3)$ \\
DFT/B3LYP & $2.41(3)$ \\
Expt. [93] & \multicolumn{2}{c}{$2.05-2.34^{c}$} \\
\hline${ }^{a}$ CASSCF(12,12)/6-31G(d) geometry from Ref. 27; \\
${ }^{b}$ Constant level shift of 0.2 a.u.; \\
${ }^{c}$ Termination with two methyl groups, -N $\left(\mathrm{CH}_{3}\right)_{2}^{+}$.
\end{tabular}

We now compare our theoretical results with gas phase photodestruction experiments which are available for the 11-cis model terminated with two methyl groups [93]. The experimental absorption spectrum displays two main peaks at 2.05 $\mathrm{eV}(610 \mathrm{~nm})$ and $3.18 \mathrm{eV}(390 \mathrm{~nm})$, which have been interpreted as the location of the vertical excitations to the two lowest singlet excited states $\left(S_{1}\right.$ and $\left.S_{2}\right)$. The lowest-energy band $\left(\mathrm{S}_{1}\right)$ displays however a broad shoulder which has a secondary peak at $2.34 \mathrm{eV}(530 \mathrm{~nm})$ and is only about 20 percent lower in intensity that the absorption maximum at $2.05 \mathrm{eV}$. It has been previously suggested [33] that the vertical transition lies in the broad shoulder at higher energies and corresponds to the secondary peak at $2.34 \mathrm{eV}$. We further propose that the adiabatic and not the vertical transition may be related to the lowest-energy feature at $2.05 \mathrm{eV}$. This interpretation of photodestruction experiments for retinal has in fact a parallel in the theoretical findings [66] and recent experimental reassessment [94] of photodestruction experiments of the photosensitive Green Fluorescent Protein chromophore. We therefore report a range of energies between $2.05 \mathrm{eV}$ and $2.34 \mathrm{eV}$ as a more conservative experimental estimate of the vertical excitation of retinal. Our DMC, single-state and MS-CASPT2 excitations are compatible with the experimental estimate, especially if we consider the remaining uncertainty on the ground-state DFT and MP2 
geometries and the fact that we did not include vibrational effects. Setting the IPEA shift to zero moves the vertical CASPT2 excitation towards the lower end of the experimental range, namely, the possible location of the adiabatic transition, and the excitation even falls below the lower bound in the case of the single-state approach. We note that we could not perform CC calculations for the 11-cis model with the available codes and that the best $\mathrm{CC} 2$ result of $2.10 \mathrm{eV}$ found in the literature [40] is about $0.20 \mathrm{eV}$ lower than the CASPT2 excitation we compute on a similar B3LYP geometry. This discrepancy is rather puzzling since the CASPT2 and CC2 excitation energies agree rather well for all smaller models, and could be due to the particular basis used in Ref. 40 or to the different response of CC2 and CASPT2 to the addition of the $\beta$-ionone ring missing in the smaller models.

\subsection{In-plane Geometrical Optimization}

We optimize the in-plane excited-state geometries of the retinal chromophore models (A, B, C, D) using the CASSCF, MS-CASPT2, CC2, CCSD, and VMC approaches. We always follow the second root in the optimization and use two roots in the SA-CASSCF and MS-CASPT2 calculations as well as in the optimization of the VMC wave functions. The CAS expansion correlates all $\pi$ electrons and an equal number of orbitals with the exception of models (A) and (B), where we include more virtual orbitals to be consistent with previous calculations [15]. As shown in Ref. 22 for model (A), a smaller active space of 6 electron in 6 orbitals yields equivalent CASSCF results. We impose the planarity of the conjugated chain by constraining the optimization to $\mathrm{C}_{s}$ symmetry and, unless otherwise stated, we start the excitedstate optimization from the DFT/B3LYP ground-state geometry.

\subsubsection{Dependence on Basis Set and Other Parameters}

In all geometrical optimizations, we employ the cc-pVDZ basis set. As shown in Figure 3.4(a) for the minimal (A) model, the effect of using the larger cc-pVTZ basis set is to systematically shorten all ground- and excited-state CASPT2 bond lengths by about $0.010-0.015 \AA$ without affecting the bond length pattern as was also previously observed in Ref. 20. Differently from the case of the excitation energies, the ANO-L-VDZP basis yields comparable bond lengths to the cc-pVDZ value, which only disagree by 0.06 and $0.07 \AA$ in the $C_{11}-C_{12}$ and $C_{12}-C_{13}$ excitedstate bonds, respectively. A similar behavior as a function of the size of the basis set is also found for the CASSCF and DFT bond lengths although the shortening in not as pronounced as for the CASPT2 results. In Figure 3.4(b), we compare the VMC results obtained with the $\mathrm{D}$ and $\mathrm{D}+$ basis sets, which are almost equal. Interestingly, the VMC results obtained with the D (cc-pVDZ) basis are very close to the CASPT2/cc-pVTZ results, so the presence of the Jastrow factor appears to compensate for the use of a smaller basis.

The VMC geometrical optimization is very sensitive to the quality of the trial 

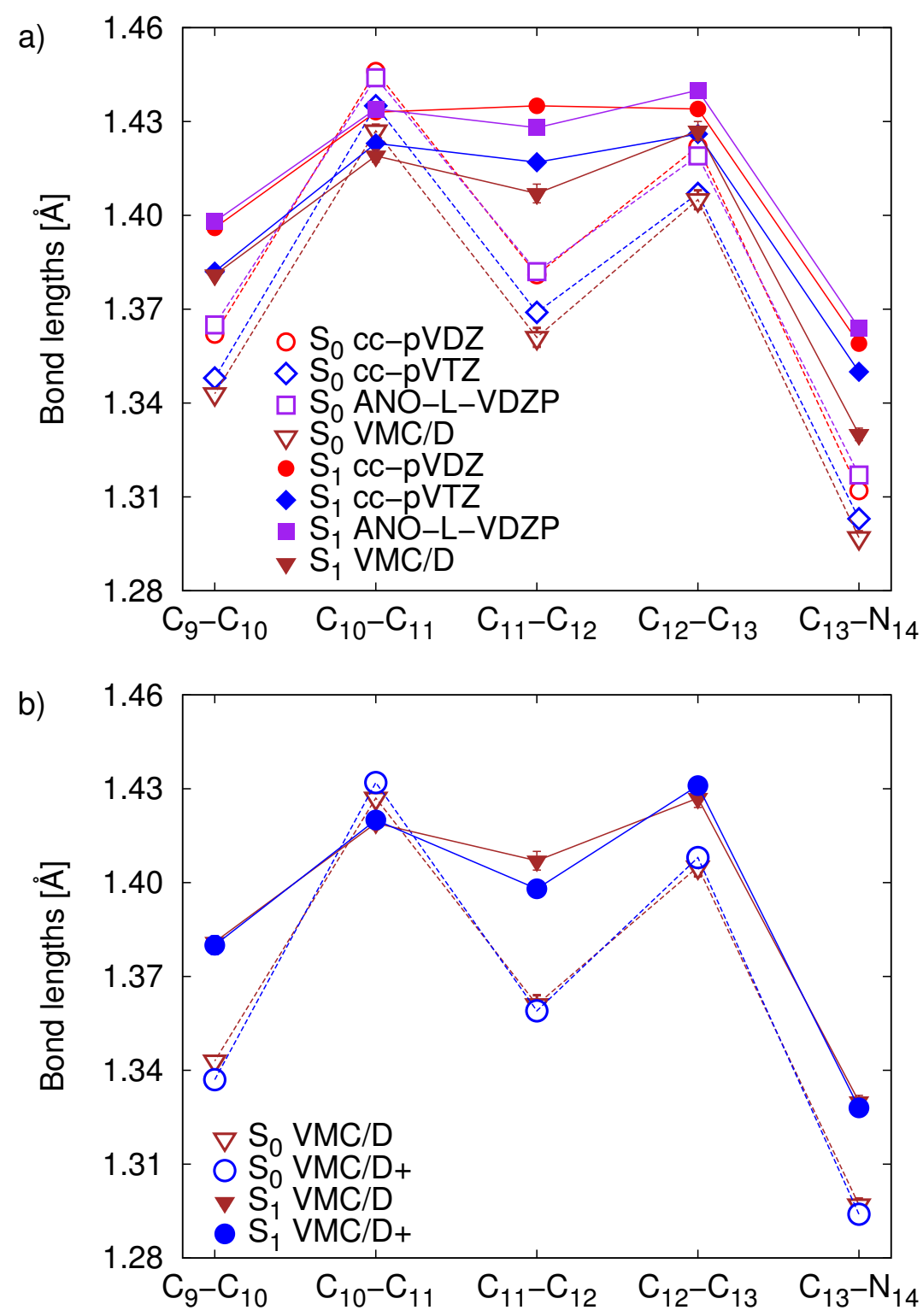

Figure 3.4: Bond lengths ( $\AA$ ) of the PSB3(0) model (A) optimized in the ground and excited states with the CASPT2 (panel a) and VMC (panel b) approaches, and different basis sets. The CASPT2 geometries are computed with the cc-pVDZ, ccpVTZ, and ANO-L-VDZP basis, and the VMC results with the D and D+ basis sets. In panel (a), the VMC/D bond lengths are also shown for comparison. Planar symmetry is imposed.

wave function as shown for model (B) in Figure 3.5. We start the optimization from the Franck-Condon region and, if we optimize only the Jastrow and CI coefficients within VMC, we obtain a VMC minimum which corresponds to the bond-inverted CASSCF geometry. On the other hand, if we include the orbital parameters in the 


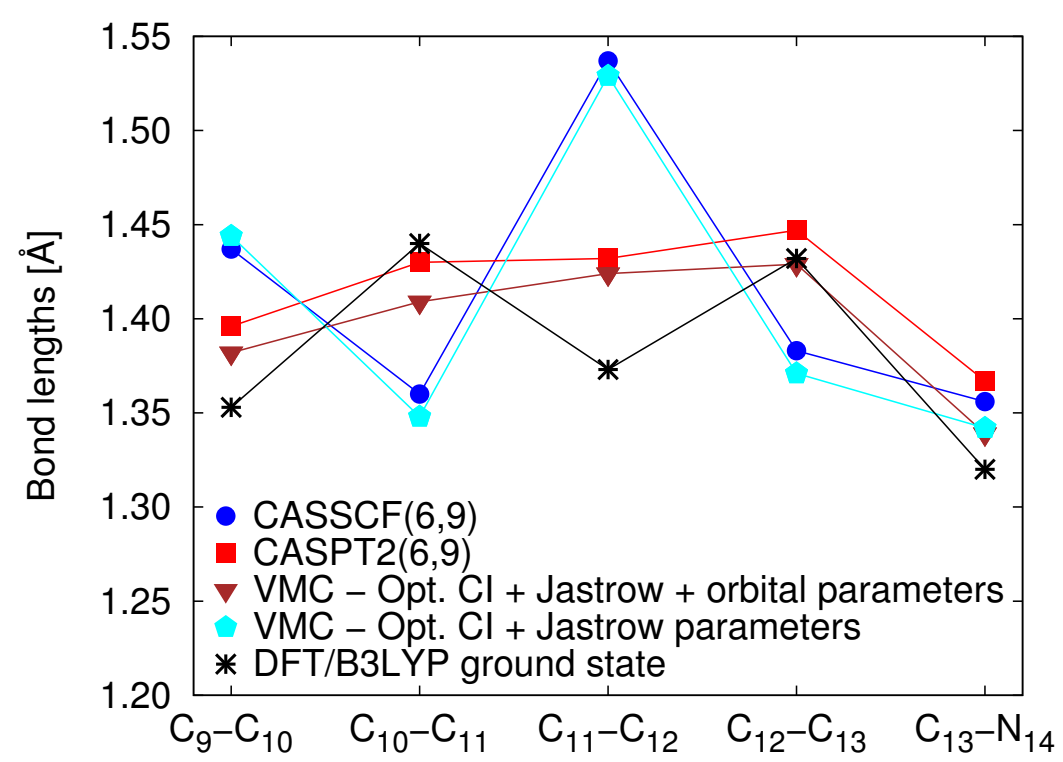

Figure 3.5: VMC bond lengths $(\AA)$ in the excited state of the PSB3(1) model (B) computed with two different wave functions. In one case, only the CI and Jastrow parameters are optimized within energy minimization in a state-average fashion while, in the other, all (orbital included) parameters are optimal. Planar symmetry is imposed.

VMC optimization, we obtain a very different VMC geometry which agrees with the minimum obtained by the other highly-correlated approaches as shown below. Thus, in the VMC geometrical relaxation, we need to optimize all wave function parameters. We also note that preliminary calculations with DMC gradients indicate that the use of DMC does not mend the behavior of VMC when the DMC gradients are computed from wave functions with only optimal Jastrow and CI parameters.

\subsubsection{Results}

To understand how the geometry of the retinal chromophore is modified upon photoexcitation, we begin with the minimal model (A) and show in Figure 3.6(a) the ground-state bond lengths as obtained with the CASSCF, CASPT2, MP2, VMC, and DFT/B3LYP approaches. All methods agree in predicting a strong single-double bond-length alternation with a short, double bond between the central carbons. The MP2 and CASPT2 geometries are almost exactly equal since the ground state is dominated by a single configuration (89\% weight) and CASPT2 is equivalent to MP2 for a single-reference CASSCF wave function. The DFT/B3LYP bond lengths deviate from the MP2 and CASPT2 values by at most $0.01 \AA$ in the two single bonds. The VMC bond lengths are shorter by about $0.015 \AA$ and this can be explained as a basis set effect as discussed above. Only the CASSCF approach is at variance with 

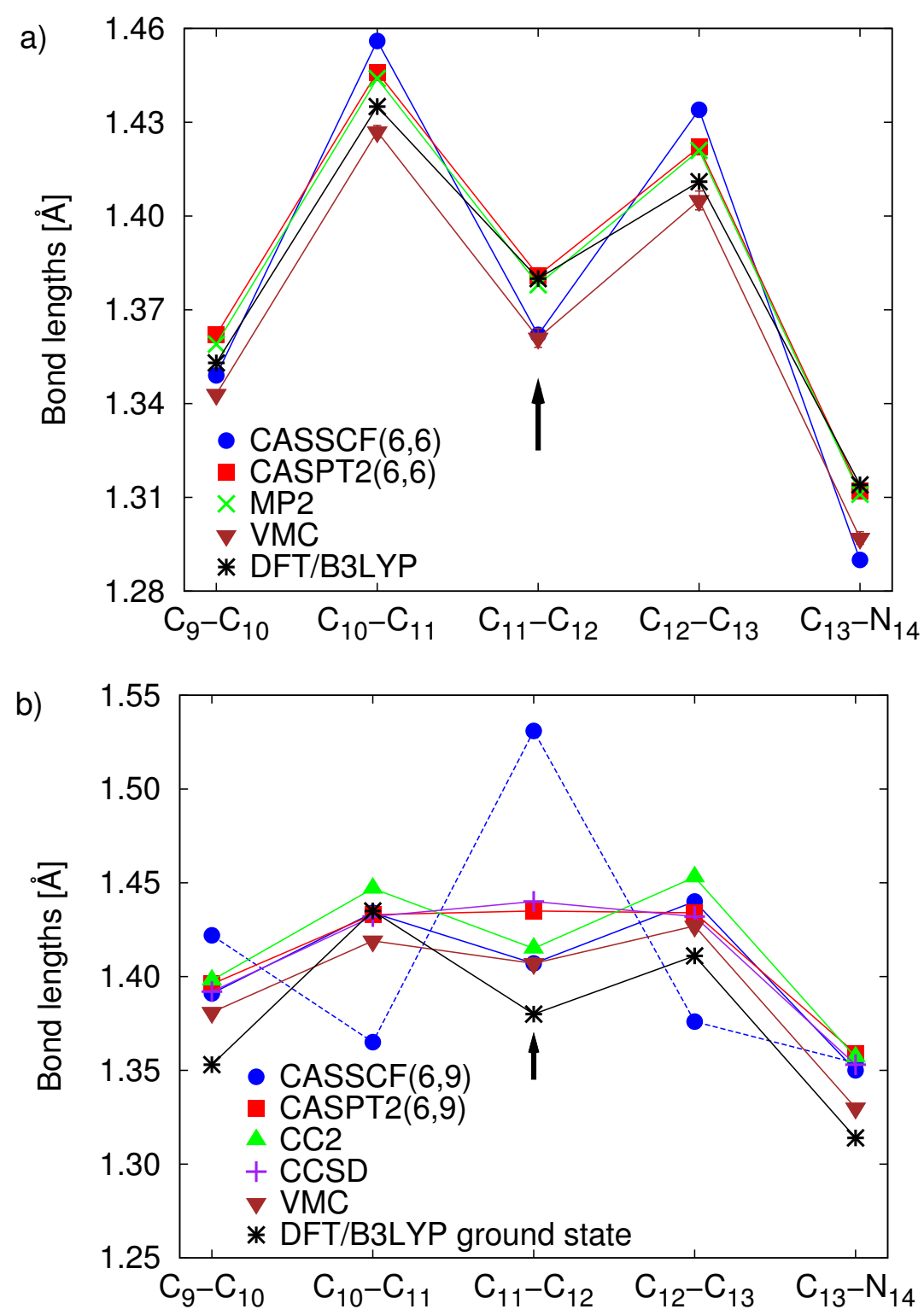

Figure 3.6: Bond lengths ( $\AA$ ) of the PSB3(0) model (A) optimized in the ground (panel a) and excited (panel b) states with the CC2, CCSD, CASSCF, CASPT2, and VMC methods. The DFT/B3LYP and MP2 ground states are also shown. The ccpVDZ basis is used and planarity imposed. CASSCF displays two minima in the excited state.

the other approaches in the sense that it exhibits a greater bond length alternation as it has also been observed for larger retinal models [20,26]. The difference between CASSCF and the other approaches is of the order of $0.01-0.02 \AA$ for model (A) but grows as the model becomes larger (see Figure SI-5 [92]). In view of these results, we find that the DFT/B3LYP approach offers a good balance between performance and computational cost for the computation of the ground state structure. 
The excited-state bond lengths of the minimal model (A) are shown in Figure 3.6(b). The CASSCF approach exhibits two almost degenerate minima while all other approaches yield only one minimum. The first CASSCF minimum (solid line) displays a lengthening of almost all bonds and a largely preserved bond-length pattern as compared to the ground state. The second CASSCF minimum (dashed line) is about $0.022 \mathrm{eV}$ higher in energy than the other CASSCF minimum, and displays a pronounced bond-length inversion with respect to the ground state. Importantly, we note that the first minimum is found when starting the optimization from the ground-state geometry while we started from a geometry biased towards bond inversion to find the second one. In addition, regardless of the starting point, we only converge to a single CASSCF minimum, corresponding to the first minimum, if the ANO-L-VDZP basis set is used instead of the cc-pVDZ basis set. For the two CASSCF minima obtained with the cc-pVDZ basis, we report the wave function character and orbitals in the SI [92].

As for the other methods, we observe that most bond lengths become longer and more similar in the excited state. The CC2 and VMC structures largely preserve the short-long bond-length pattern of the ground state as observed for the first CASSCF minimum, while CASPT2 and CCSD give three middle bonds of almost equal length. At the CASPT2 level, we also investigated extensively the existence of a bond-inverted minimum by starting the excited-state optimization from geometries biased towards bond inversion, but could not locate a second minimum. Our CASSCF and CASPT2 results are consistent with the study by Page and Olivucci [20] using the 6-31G(d) basis set.

Surprisingly, adding a methyl group to the minimal model (A) to generate model (B) has profound effects on the bond lengths as shown in Figure 3.7. In particular, there is now only one CASSCF minimum which exhibits a pronounced bond length inversion as compared to the ground state and is at variance with the results obtained with all other approaches. The differences among the results obtained with the other methods is instead significantly smaller. The CC2 geometry of model (B) is similar to model (A) with a lengthening of most bonds and a largely preserved bond-length pattern with respect to the ground state. Similarly to model (A), CASPT2 yields close to equal bond lengths for the three middle bonds with the $\mathrm{C}_{12}-\mathrm{C}_{13}$ bond being the longest while CCSD gives the middle $\mathrm{C}_{11}-\mathrm{C}_{12}$ bond as being slightly larger. The VMC minimum displays similar bond length pattern as CASPT2 but shorter absolute bond lengths which can be explained as a basis set effect as explained above.

When going to larger models, we find that CASSCF yields only one minimum where the short-long bond-length pattern is inverted with respect to the ground state as in the case of model (B). In Figure 3.8, we show the excited-state bond lengths for model $(C)$ and observe that the CASSCF minimum with bond-length inversion is at variance with all other approaches. The CASPT2 and CC2 are very close to each other and exhibit a largely preserved bond length pattern and overall lengthening of most bonds with respect to the ground state. The CCSD geometry displays no distinct bond-length pattern and an overall lengthening of most bonds and the VMC gives a similar bond-length pattern as CCSD but shorter bond lengths as seen above. 


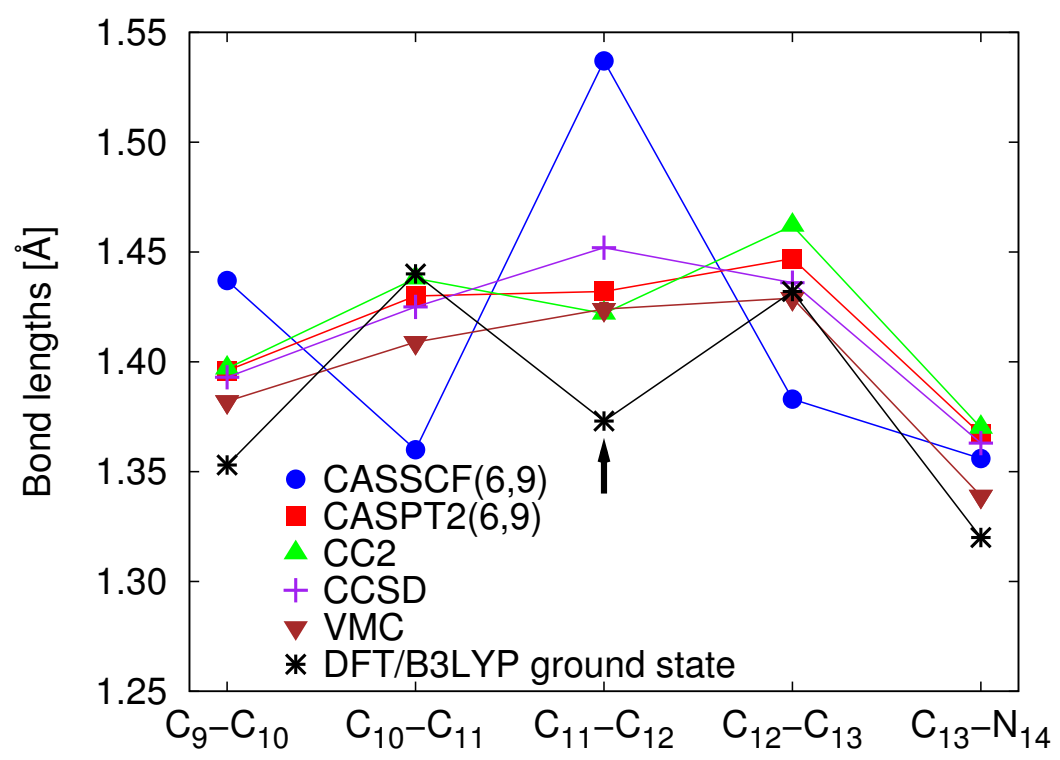

Figure 3.7: Bond lengths ( $\AA$ ) of the PSB3(1) model (B) optimized in the excited state with the CC2, CCSD, CASSCF, and CASPT2 methods. The DFT/B3LYP groundstate bond lengths are also shown. The cc-pVDZ basis is used and planar symmetry imposed. Differently from model (A) without the methyl (Figure 3.6), CASSCF only displays here one minimum.

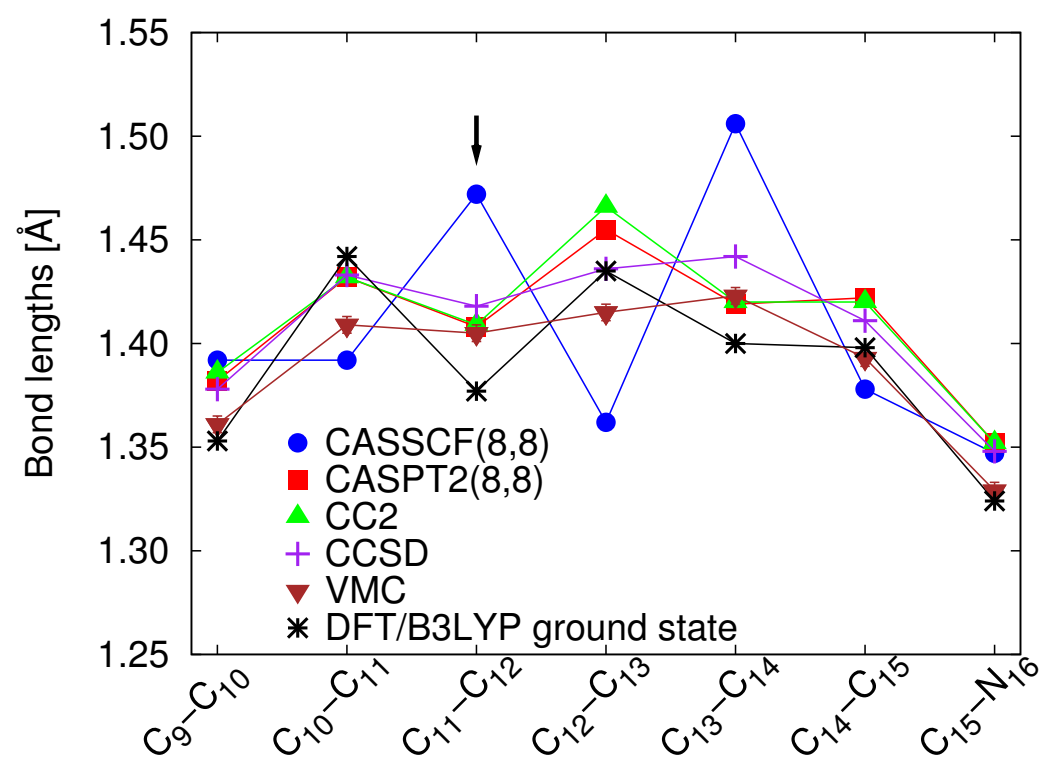

Figure 3.8: Bond lengths ( $\AA$ ) of the PSB4(1) model (C) optimized in the excited state with the CC2, CCSD, CASSCF, CASPT2, and VMC methods. The DFT/B3LYP ground-state bond lengths are also shown. The cc-pVDZ basis is used and planar symmetry imposed. 


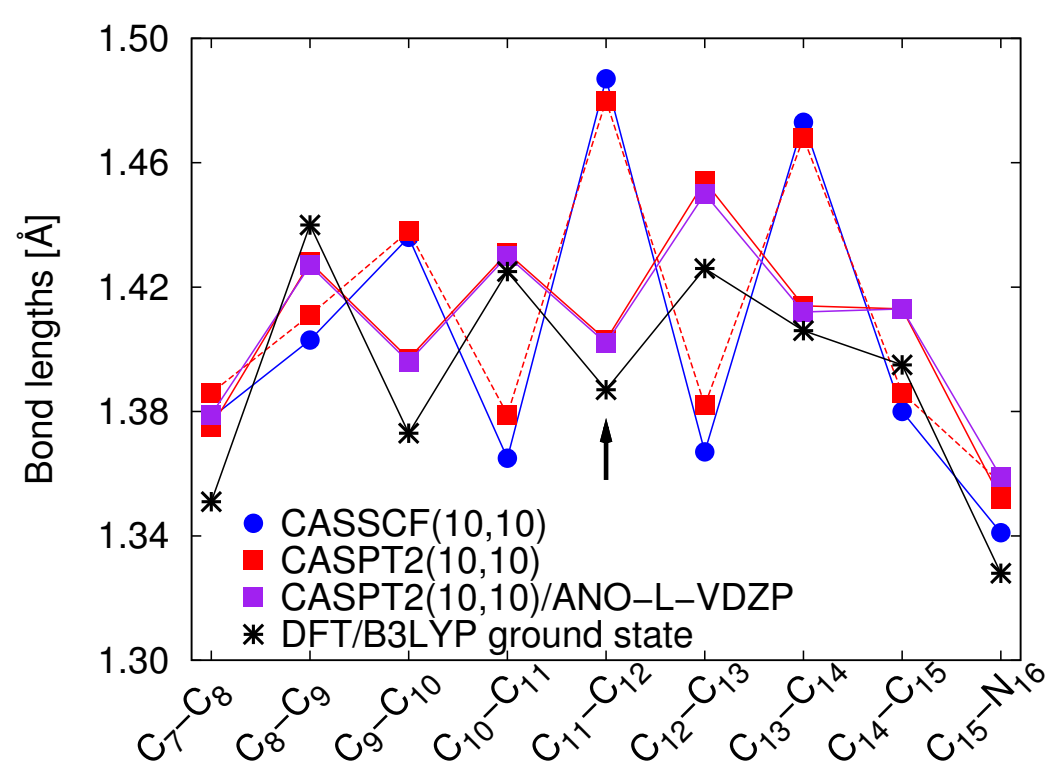

Figure 3.9: Bond lengths ( $\AA$ ) of the PSB5(1) model (D) optimized in the excited state with the CASSCF and CASPT2 methods and the cc-pVDZ basis. We also show the CASPT2/ANO-L-VDZP results. CASPT2 displays two minima in the excited state with the cc-pVDZ basis and only one minimum with the ANO-L-VDZP basis. The DFT/B3LYP ground-state bond lengths are also shown. Planar symmetry is imposed.

In Figure 3.9, we show the excited-state bond lengths of model (D) which has the full conjugated chain of the retinal chromophore and only misses the $\beta$-ionone ring. For this model, we only show the bond lengths obtained with the CASSCF and CASPT2 approaches. In addition, we also show CASPT2 results obtained with the ANO-L-VDZP basis set. As for models (B) and (C), CASSCF gives a structure characterized by bond-length inversion with respect to the ground state. However, it is now the CASPT2 approach which gives two profoundly different minima. The first CASPT2 minimum (solid line) is similar to the CASPT2 geometry of model (C) with a preserved bond length pattern and overall lengthening of most bonds as compared to the ground state. The second CASPT2 minimum (dashed line) is very close to the CASSCF geometry and is about $0.045 \mathrm{eV}$ higher in energy than the first CASPT2 minimum. Importantly, we note that the first CASPT2 minimum is found when starting from the ground-state geometry while the second CASPT2 minimum is reached when starting from the CASSCF excited-state geometry. Moreover, the existence of this second minimum is dependent on the choice of the basis: The two CASPT2 minima obtained with the cc-pVDZ basis are also found when the 6-31G(d) basis set is used (not shown in the Figure) while only the first CASPT2 minimum with no bond length inversion is obtained regardless of the starting geometry when the ANO-L-VDZP basis set is used. These results seem to indicate that 
the bond-inverted CASPT2 structure is a spurious local minimum with no chemical significance, which is not reached when the optimization is started from the ground-state structure, that is, upon photoexcitation. We finally observe that a previous CASSCF and CASPT2 study by Page and Olivucci [20] using the 6-31G(d) basis set reports an excited-state CASPT2 structure of model (D) characterized by bond inversion. This finding can be easily explained by the fact that they started the CASPT2 geometrical optimization from the excited-state CASSCF minimum and were thus not able to reach the other minimum.

In summary, we see that the CASSCF excited-state geometries are at variance with the CASPT2, CC, and QMC results with the exception of the minimal model (A) where CASSCF displays two minimum structures, one of which is in agreement with the geometries obtained by the other approaches. The minimal model appears however to be a special case since the addition of a single methyl group in model (B) changes the picture and breaks the agreement between CASSCF and the other approaches. The inadequacy of CASSCF in describing in-plane excited structures of the retinal chromophore is also apparent from the results obtained for all the larger models.

\subsection{Out-of-Plane Relaxation}

\subsubsection{Minimum Energy Paths}

We determine the excited-state MEP of the retinal models (B) and (C) using the CASSCF and CASPT2 approaches. Ground- and excited-state CASSCF MEPs have previously been calculated for several retinal models $[15,16,18,22,33]$ and the common assumption is that the effect of dynamical correlation can be in part recovered by simply computing the CASPT2 energy on the final CASSCF geometries (CASPT2//CASSCF). Our aim is here to assess the validity of this assumption for the retinal chromophores by comparing the CASSCF and CASPT2 MEPs. To the best of our knowledge, the CASPT2 method has not been used to determine MEPs for the retinal models since CASPT2 energy gradients are substantially more expensive than CASSCF ones and still considered too costly for the routine investigation of these systems $[38,55]$. In the literature, we only found a CASPT2 study performing a constrained excited-state potential energy surface scan for the minimal model (A) [41].

The MEP calculations are performed using the steepest descent path optimization scheme implemented in MOLCAS 7.2 and described in Ref. 95. The procedure consists of a series of constrained geometrical optimizations in mass-weighted coordinates, and yields the intrinsic reaction path. In each optimization, the potential energy is minimized on a hypersphere of a chosen radius, centered at a given reference structure. The CASSCF and CASPT2 ground-state geometries define the Franck-Condon point and initial reference structure for the corresponding MEP calculations. The radius of the hypersphere is either 0.06 or 0.1 a.u. for model (B) and 
0.1 a.u. for model (C). Upon convergence of the constrained geometrical optimization, the obtained minimum structure on the hypersphere is taken as new reference structure, and the procedure is iterated. As in the planar optimizations, the state averaging in the CASSCF and CASPT2 includes only the ground- $\left(\mathrm{S}_{0}\right)$ and first excited-state $\left(\mathrm{S}_{1}\right)$ since the next state is significantly higher in energy and does not play an active role (see SI [92]).

We define the torsional angle $\theta$ as the $\mathrm{C}_{10}-\mathrm{C}_{11}-\mathrm{C}_{12}-\mathrm{C}_{13}$ dihedral angle and the torsional angle $\gamma=180^{\circ}-\phi$ where $\phi$ is the $\mathrm{C}_{11}-\mathrm{C}_{12}-\mathrm{C}_{13}-\mathrm{C}_{14}$ dihedral angle and $\gamma$ is taken in the range from $-180^{\circ}$ to $180^{\circ}$. Both torsional angles have a value of $0^{\circ}$ in the ground state and indicate the deviation for planarity. These angles correspond to the torsional motion around the $\mathrm{C}_{11}-\mathrm{C}_{12}$ and $\mathrm{C}_{12}-\mathrm{C}_{13}$ bonds which are double and single in the ground state, respectively. We note that geometries corresponding to the angles $(\theta, \gamma)$ and $(-\theta,-\gamma)$ are equivalent since the molecules are planar in the ground state and there is no preferential direction for torsion.

In Figure 3.10, we show the results from the MEP calculation for model (B) and report the energies, the bond lengths for the formal double and single bonds along the conjugated chain, and the torsional angle $\theta$ for the central $\mathrm{C}_{11}-\mathrm{C}_{12}$ cis bond. The CASSCF MEP is characterized by two sequential modes. The initial relaxation is towards a planar structure similar to the CASSCF $\mathrm{C}_{s}$ minimum discussed above, which exhibits bond-length inversion with respect to the ground state with the central $\mathrm{C}_{11}-\mathrm{C}_{12}$ bond being the longest in the excited state. This in-plane motion is followed by a torsion around the central bond towards an angle $\theta$ of about $65^{\circ}$, where a conical intersection region is encountered and the excited-state MEP is stopped. The CASSCF MEP is barrierless while there is a small barrier of about $0.1 \mathrm{eV}$ in the CASPT2//CASSCF energies.

The CASPT2 MEP is distinctly different from the CASSCF one even though the final outcome of the photoisomerization process is similar. The first difference is that the initial planar relaxation is towards a structure similar to the CASPT2 $\mathrm{C}_{s}$ minimum which is therefore not characterized by bond inversion. The three middle bonds become almost equal and the $\mathrm{C}_{12}-\mathrm{C}_{13}$ bond, which is long in the ground state, is the longest in the excited state. The subsequent torsional motion is around the central $\mathrm{C}_{11}-\mathrm{C}_{12}$ bond where we observe a plateau in the excitedstate energy up to an angle $\theta$ of about $22^{\circ}$. When $\theta$ is about $17^{\circ}$, the three middle bonds begin to change dramatically: The central $C_{11}-C_{12}$ bond lengthens while the two neighboring bonds shorten, so their lengths become similar to the ones of the CASSCF MEP. The excited-state energy starts then decreasing at a faster pace and the torsional motion continues towards $\theta \approx 69^{\circ}$ where a conical intersection region is encountered and the excited-state MEP is stopped. A similar behavior is observed in the constraint excited-state optimization of the minimal model (A) in Ref. 41, where an energy plateau is observed for $\theta$ between $0^{\circ}$ and $25^{\circ}$, followed by a sudden drop in the energy and change in geometry between $25^{\circ}$ and $30^{\circ}$. In addition, studies on the minimal model (A) have found that conical intersection geometries obtained with CASSCF and CASPT2 are very similar [19,20,23]. This is consistent with the results obtained here as the CASSCF and CASPT2 MEPs show similar structures 


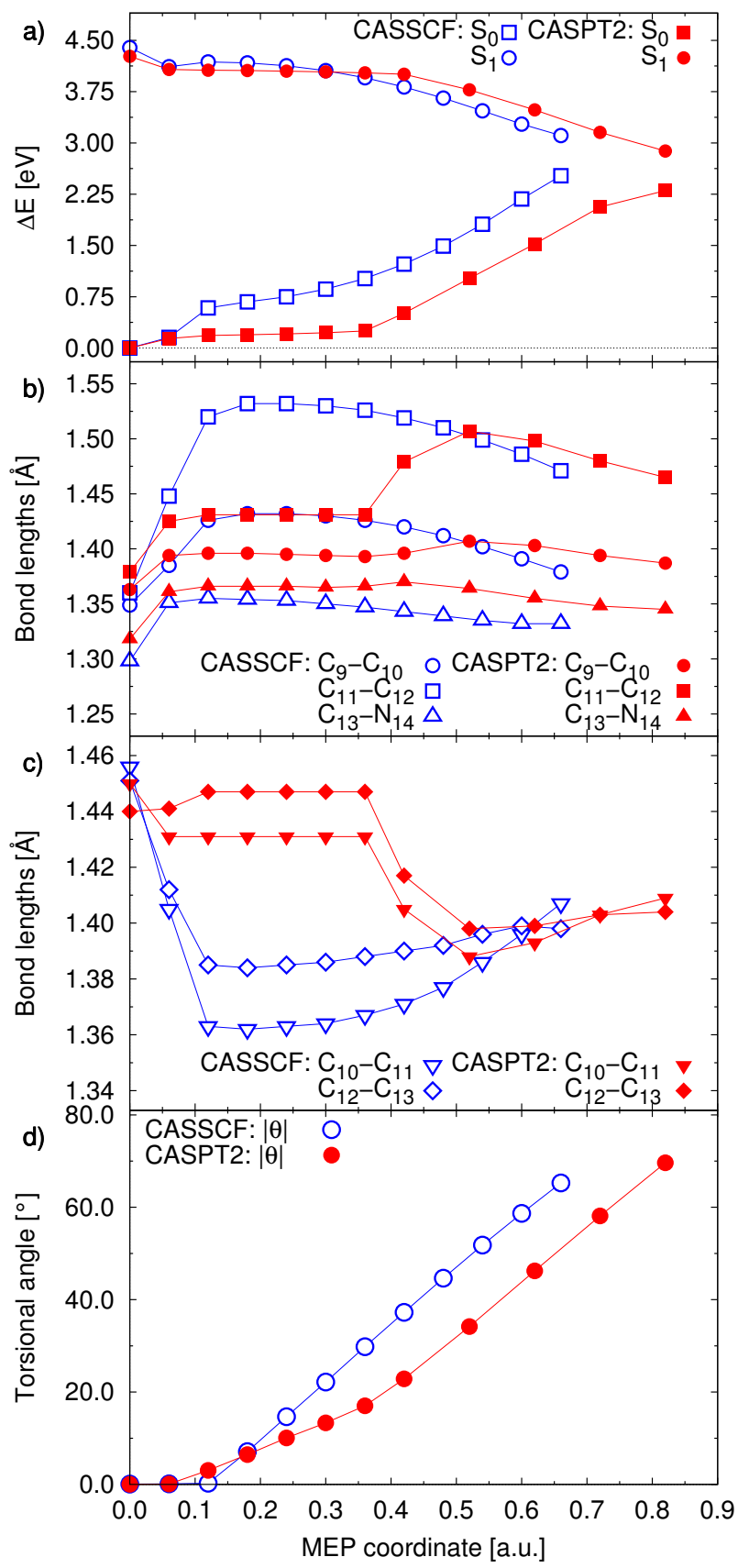

Figure 3.10: CASSCF and CASPT2 excited-state MEPs for the PSB3(1) model (B), obtained with a CAS $(6,9)$ expansion and the cc-pVDZ basis. We report the CASPT2//CASSCF and CASPT2//CASPT2 ground- and excited-state energies (a), the bond lengths for formal double (b) and single bonds (c), and the absolute value of the torsional angle $\theta$ around the central $\mathrm{C}_{11}-\mathrm{C}_{12}$ bond (d). All energies are relative to the ground-state energies of the CASSCF and CASPT2 ground-state geometries, which are the starting point of the corresponding MEPs. 
near the conical intersection.

To investigate the effect of lengthening the conjugated chain, we compute the MEP of model (C) as shown in Figure 3.11. The CASSCF and CASPT2 approaches give a different isomerization mechanism and the relevant torsional angles are not only $\theta$ around the $\mathrm{C}_{11}-\mathrm{C}_{12}$ bond (formal double) but also $\gamma$ around the $\mathrm{C}_{12}-\mathrm{C}_{13}$ bond (formal single). The CASSCF MEP is similar to the one of model (B) and is characterized by two sequential modes, namely, an initial in-plane bond-length inversion followed by a torsional motion around the $\mathrm{C}_{11}-\mathrm{C}_{12}$ bond until the conical intersection region is encountered at $\theta \approx 88^{\circ}$. There is also a small torsion around the $\mathrm{C}_{12}-\mathrm{C}_{13}$ bond with an angle $\gamma \approx 13^{\circ}$ at the end of the MEP. Differently from model (B), the CASPT2//CASSCF excited-state energies show no barrier.

The CASPT2 MEP is rather different from the CASSCF one. The initial relaxation is towards a planar structure which is similar to the CASPT2 $\mathrm{C}_{s}$ minimum and exhibits a largely preserved bond-length pattern with respect to the ground state and an overall lengthening of most bonds. This in-plane motion is followed by a concerted increase of $\theta$ (also active in the CASSCF isomerization) and $\gamma$ up to a MEP coordinate of 0.5 a.u. Beyond this point, $\gamma$ keeps increasing while $\theta$ changes only slightly, so the molecule is twisting only around the $\mathrm{C}_{12}-\mathrm{C}_{13}$ bond (formal single) while all bond lengths remain almost constant. At a MEP coordinate of 1.5 a.u. $\left(\gamma \approx-49^{\circ}\right)$, a barrier is encountered and the MEP optimization cannot proceed further. Both the ground- and excited-state energies vary very little along the whole MEP and both states display a long plateau. At the final MEP coordinate, the excited-state energy is only $0.20 \mathrm{eV}$ lower than the Franck-Condon point and the ground-state energy higher by about $0.44 \mathrm{eV}$, so the vertical excitation has decreased from $3.44 \mathrm{eV}$ to $2.80 \mathrm{eV}$.

In order to compare the CASSCF and CASPT2 isomerization mechanisms with the $\mathrm{CC} 2$ results, we also perform straight geometrical excited-state optimization with all three approaches since the code we use to perform CC2 calculations does not have the capability of computing MEP. For consistency, all optimizations are started from the DFT/B3LYP ground-state geometries. For model (B), all the approaches yield isomerization around the central $\mathrm{C}_{11}-\mathrm{C}_{12}$ bond and proceed towards the same final point in the conical intersection region. However, from the CASSCF and CASPT2 MEP results, we know that the isomerization proceed rather differently even though the final structures are equivalent. Therefore, we cannot infer too much about the behavior of CC2 from the agreement of the method on the final structure of model (B) but proceed with model $(\mathrm{C})$ where the final outcome of the CASSCF and CASPT2 MEP are distinctively different.

We show the optimal CC2 and CASPT2 excited-state structures of model (C) in Figure 3.12. We observe that CC2 isomerize around the $\mathrm{C}_{12}-\mathrm{C}_{13}$ bond as CASPT2 while CASSCF is consistent with the MEP behavior and yields isomerization around the $\mathrm{C}_{11}-\mathrm{C}_{12}$ bond (not shown in the Figure). The CASPT2 optimal geometry has a torsional angle $\gamma=43.6^{\circ}$ and is energetically between the MEP geometries at 1.3 and 1.4 a.u. Even though the isomerization is around the same bond, the optimal CC2 torsional angle of $\gamma=100.1^{\circ}$ is however significantly different from 


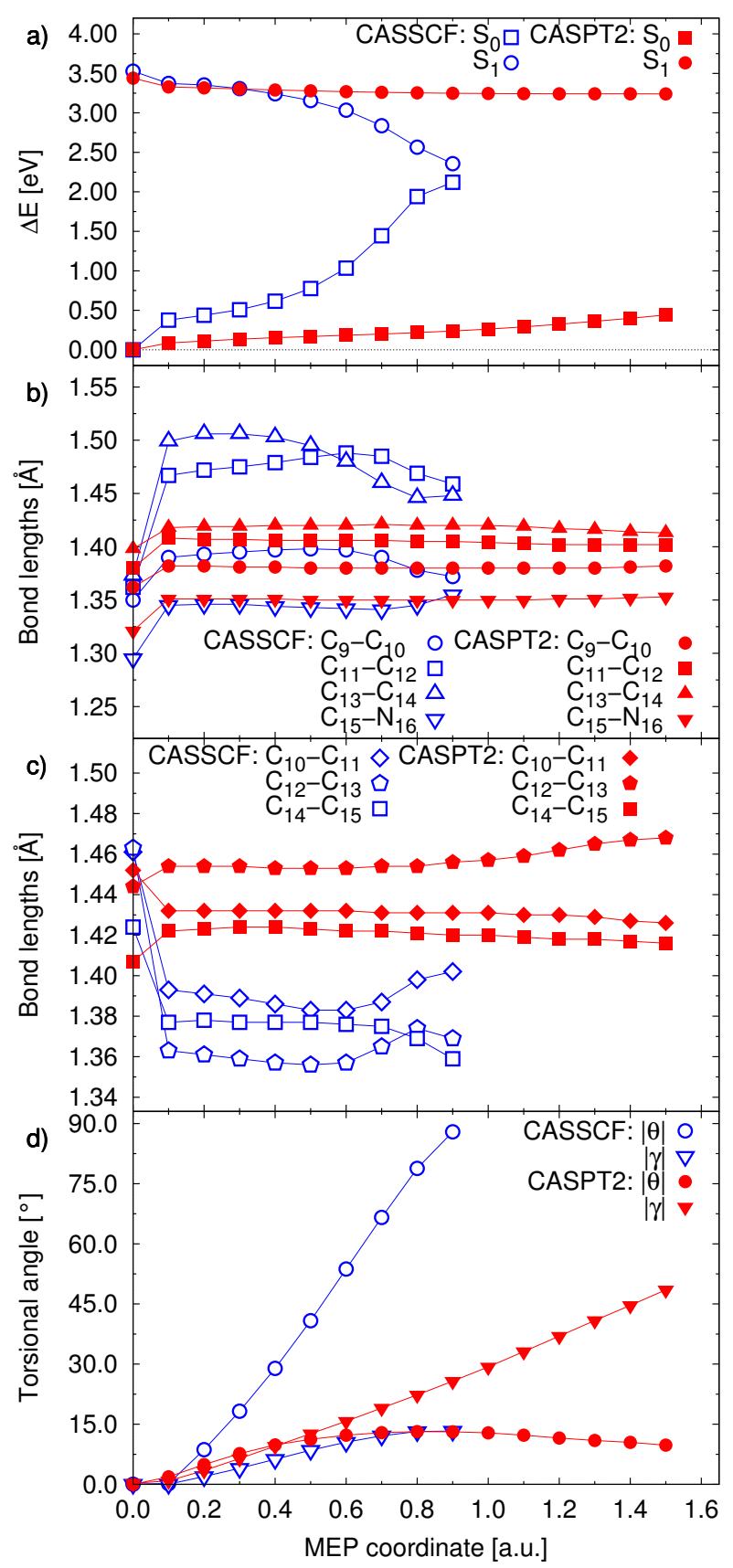

Figure 3.11: CASSCF and CASPT2 excited-state MEPs for the PSB4(1) model (C), obtained with a CAS $(8,8)$ expansion and the cc-pVDZ basis. We report the CASPT2//CASSCF and CASPT2//CASPT2 ground- and excited-state energies (a), the bond lengths for formal double (b) and single bonds (c), and the absolute values of the torsional angles $\theta$ and $\gamma$ around the $\mathrm{C}_{11}-\mathrm{C}_{12}$ and the $\mathrm{C}_{12}-\mathrm{C}_{13}$ bond, respectively (d). For CASSCF, $\theta<0^{\circ}$ and $\gamma<0^{\circ}$ while, for CASPT2, $\theta>0^{\circ}$ and $\gamma<0^{\circ}$. All energies are relative to the ground-state energies at the CASSCF and CASPT2 ground-state geometries, which are the starting point for the corresponding MEPs. 


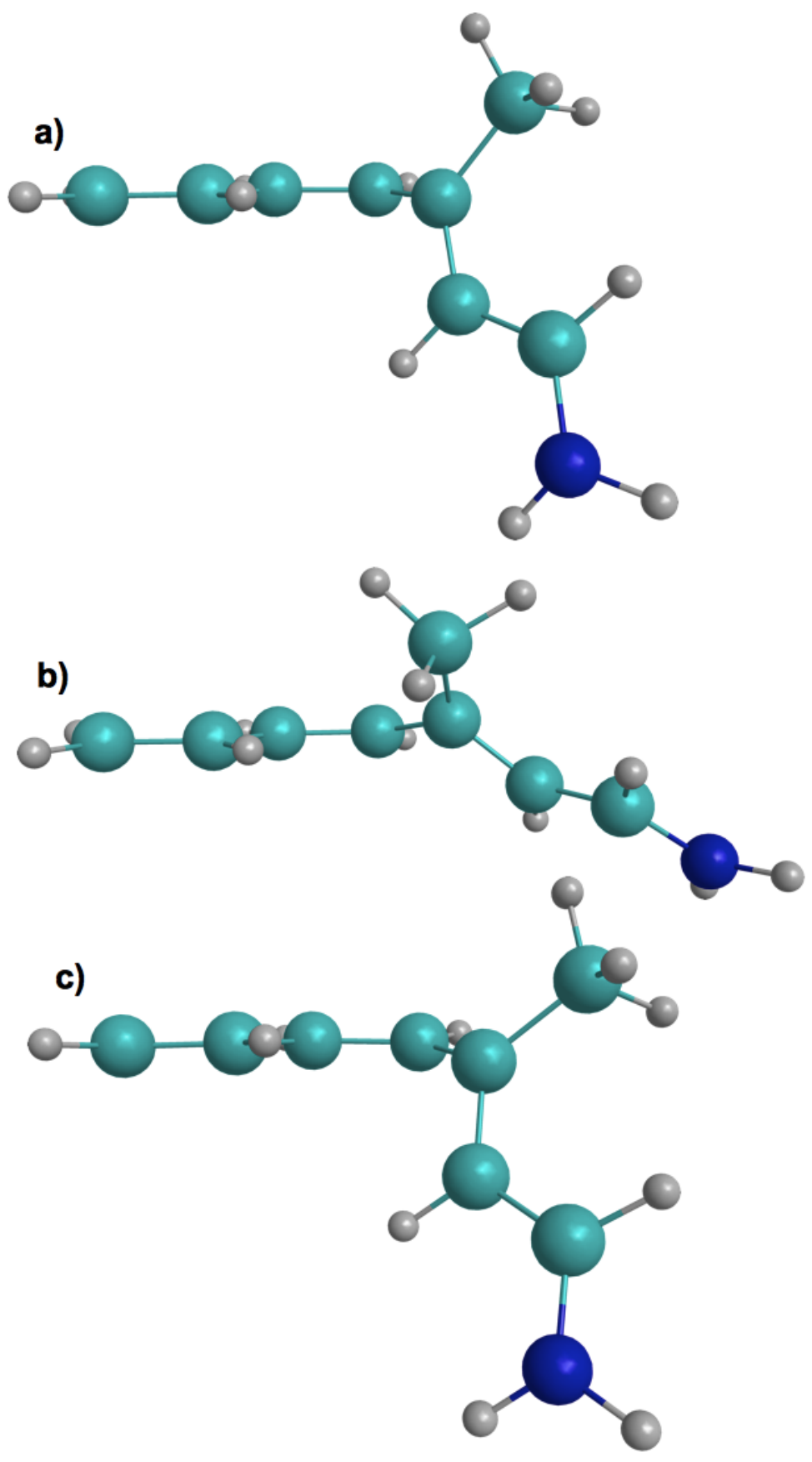

Figure 3.12: CC2 (a) and CASPT2 (b) excited-state optimal structures of the PSB4(1) model (C), obtained by starting the optimization from the DFT/B3LYP ground-state geometry. The $\mathrm{CC} 2$ torsional angles are $\gamma=100.1^{\circ}$ and $\theta=2.6^{\circ}$ while CASPT2 yields $\gamma=43.6^{\circ}$ and $\theta=-10.5^{\circ}$. The CASPT2 structure (c) is obtained by starting the optimization from the constrained structure just beyond the barrier $\left(\gamma=75^{\circ}\right)$ in Figure 3.13, and has angles $\gamma=112.7^{\circ}$ and $\theta=8.1^{\circ}$. 


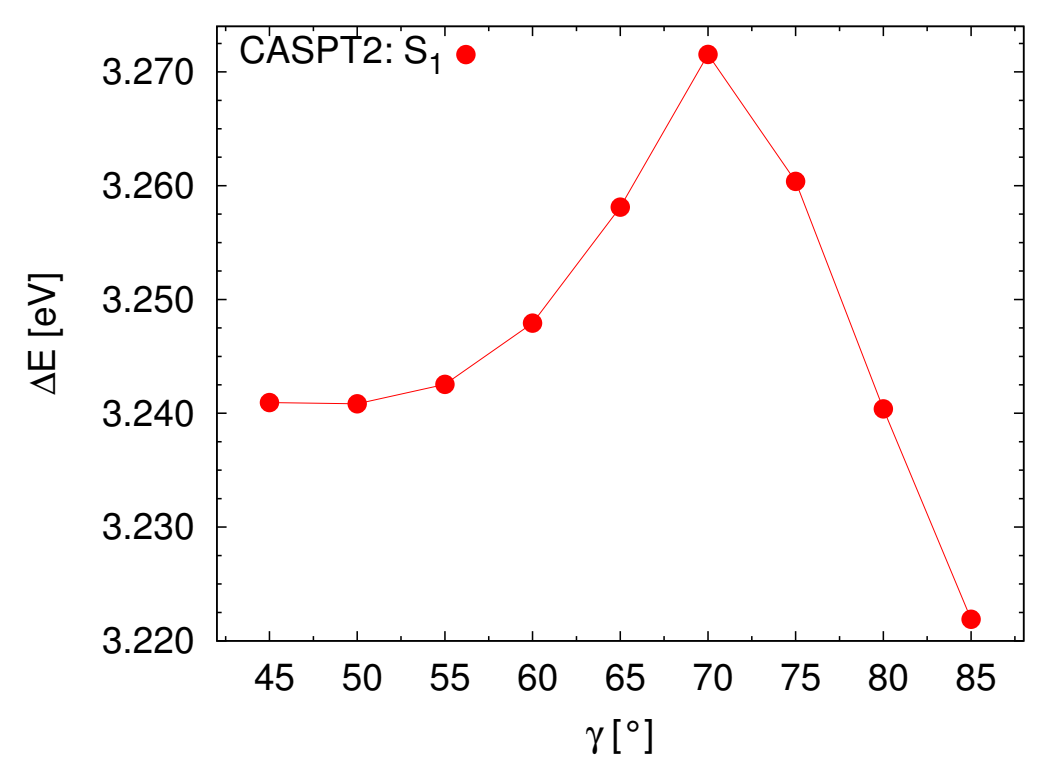

Figure 3.13: CASPT2 excited-state energies of the PSB4(1) model (C) optimized at constrained torsional angles, $\gamma$, from $45^{\circ}$ to $85^{\circ}$. The energy is shown relative to the ground-state value at the starting point of the CASPT2 MEP (Figure 3.11). A CAS $(8,8)$ expansion and the cc-pVDZ basis set are used.

the CASPT2 value. To understand this difference, we investigate the possible existence of a barrier in the CASPT2 potential energy surface and perform a constrained excited-state geometrical optimization in CASPT2 by varying the angle $\gamma$ between $45^{\circ}$ to $85^{\circ}$. The resulting excited-state energies are shown in Figure 3.13 and display a small barrier of about $0.03 \mathrm{eV}$. If we perform an excited-state CASPT2 optimization starting from the constrained structure just beyond the barrier, we recover a minimum excited-state structure which has a torsional angle of $\gamma=112.7$ (Figure 3.12(c)) and is in much closer agreement with the CC2 optimal geometry. The CASPT2 excited-state energy is only $0.09 \mathrm{eV}$ lower than the value for the minimal structure at $\gamma=43.6^{\circ}$. An analysis of the CASPT2 geometries along the constrained path of Figure 3.13 reveals that the origin of the barrier is due to steric interactions of the methyl group with the nearby hydrogens since the main difference between the geometries before and after the barrier is a small rotation of the methyl group. We also note that a previous CC2 investigation on model (C) without the methyl group [PSB4(0)] found a small barrier of $0.01 \mathrm{eV}$ at $\gamma \approx 30^{\circ}$ and an absolute minimum at about $100^{\circ}$ [29]. Therefore, the apparent presence/absence of a barrier in the CASPT2/CC2 optimization may possibly be due to the particular geometrical optimization algorithm used in the different codes or to slightly different initial configurations in the optimization procedure. 


\subsubsection{Reactive versus Non-reactive Paths}

The CASPT2 MEP of the retinal model (C) gives isomerization around a single bond, does not lead to a conical intersection region, and corresponds to a nonreactive path. To investigate whether a rotation around a double bond may give a reactive path and lead to a photoproduct, we optimize the excited-state CASPT2 geometry of model $(\mathrm{C})$ at constrained torsional angles, $\theta$, around the $\mathrm{C}_{11}-\mathrm{C}_{12}$ cis bond, and show the results in Figure 3.14.

At $\theta=0^{\circ}$, the molecule is unstable towards single-bond rotation, which is not surprising since the CASPT2 MEP gives isomerization around the same single bond and is always characterized by small values of the angle $\theta$ (less than $10^{\circ}$ ). The resulting constrained geometry has an angle $\gamma$ of about $51^{\circ}$ and is in fact very similar to the last point of the CASPT2 MEP. If we increase $\theta$ from $0^{\circ}$ to $35^{\circ}$, the angle $\gamma$ diminishes while the bond lengths become closer to the values in the initial part of the CASPT2 MEP. Concurrently, the excited-state energy rises and displays a small barrier of about $0.06 \mathrm{eV}$, which peaks at $\theta=35^{\circ}$. The barrier is overcome at $\theta=40^{\circ}$ where we suddenly observe bond inversion and a large increase in the ground-state energy and decrease in the excited-state energy. The degree of bond inversion is however not as pronounced as in the CASSCF MEP and the geometries are characterized by a larger residual rotation around the single bond. If we further increase $\theta$, the excitation energy continues to decrease and we encounter a conical intersection region. The CASPT2 isomerization around the double bond corresponds therefore to a reactive path which is characterized by a small barier and eventually leads to a conical intersection region whose topology is rather similar to the CASSCF one.

To assess the behavior of the $\mathrm{CC}$ approach, we also perform constrained $\mathrm{CC} 2$ optimization around the double bond. The CC2 optimization at small values of $\theta$ leads to a single-bond rotation with very large values of $\gamma\left(\right.$ greater than $90^{\circ}$ ). This is compatible with the previous observation that the small steric barrier observed in CASPT2 (see Figure 3.13) is practically absent in the single-bond isomerization at $\mathrm{CC} 2$ level. If we increase $\theta$ up to $60^{\circ}$ and always start the optimization from the optimal constrained geometry at the previous angle, we cannot sufficiently reverse the large rotation around the single bond and the excited-state energy increases instead of decreasing. To assess the existence of a path leading to a conical intersection, we follow therefore a different procedure and simply compute the $\mathrm{CC} 2$ energies on the optimal constrained CASPT2 geometries of Figure 3.14. We find that the groundand excited-state CC2 energies are in very good agreement with the CASPT2 values up to $\theta=45^{\circ}$. As expected and also discussed in Ref. 37, CC2 encounters convergence problems at larger values of $\theta$ as the system is approaching the conical intersection region. Consequently, the use of CC2 confirms the existence of a reactive path which corresponds to double-bond rotation, displays a small barrier, and leads to lower excited-state energies. However, the approach is not suitable to follow the system through the conical intersection towards a photoproduct. 


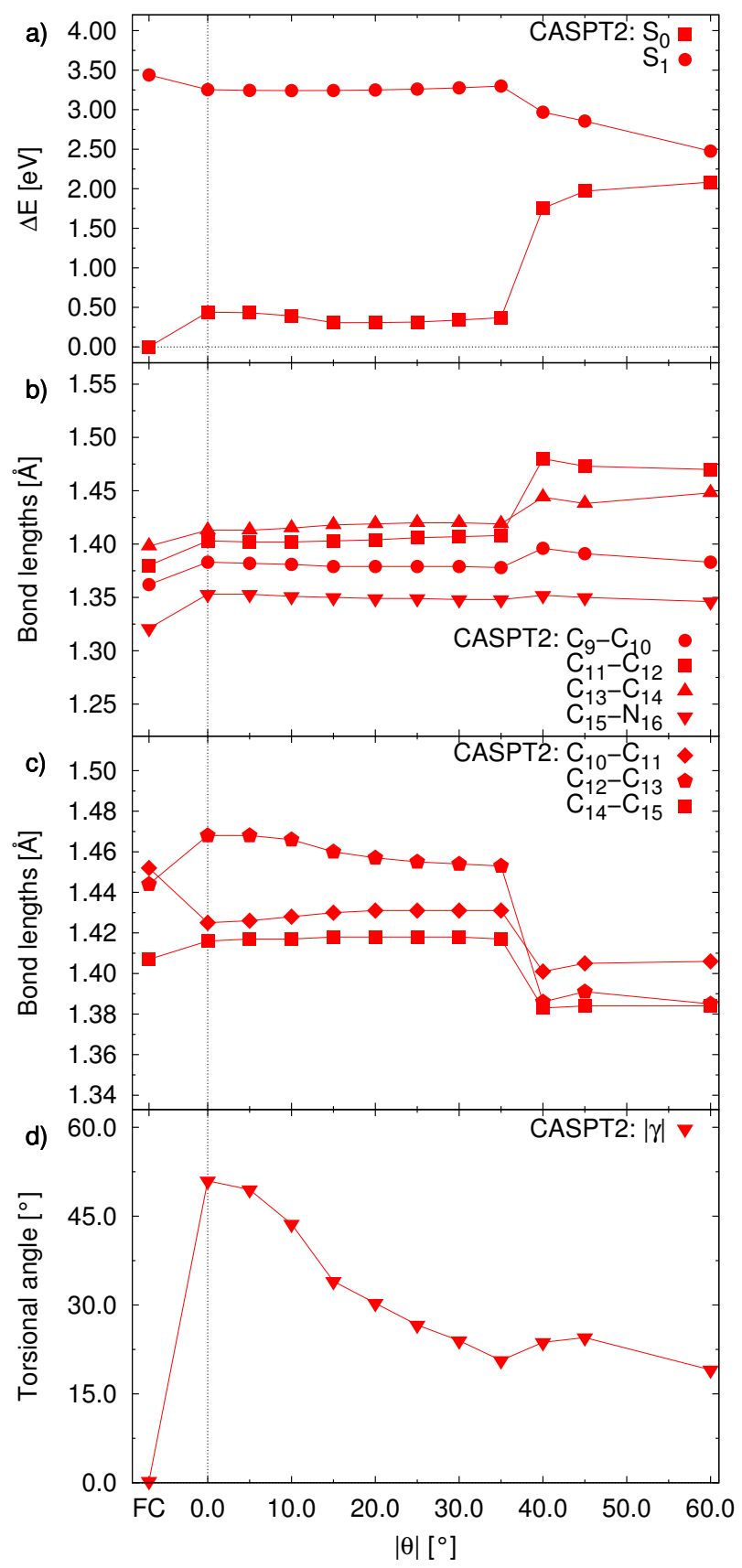

Figure 3.14: CASPT2 excited-state optimization of the PSB4(1) model (C) at constrained torsional angles, $\theta$, from $0^{\circ}$ to $60^{\circ}$. We report the CASPT2//CASPT2 ground- and excited-state energies (a), the bond lengths for formal double (b) and single bonds (c), and the absolute value of the torsional angle $\gamma$ around the $\mathrm{C}_{12}-\mathrm{C}_{13}$ bond (d). The quantities computed at the CASPT2 Franck-Condon (FC) point are also shown in all panels. A CAS $(8,8)$ expansion and the cc-pVDZ basis set are used. For the torsional angle, $\theta<0^{\circ}$ and $\gamma>0^{\circ}$. 


\subsection{Discussion and Conclusions}

We have presented a systematic investigation of model retinal chromophores in the gas phase with special emphasis on geometrical relaxation in the excited state. One aim of the work is to assess the relative performance of very diverse computational approaches as CASSCF, CASPT2, CC, and QMC in describing conformational changes in the excited states. The other major goal is to determine the validity of the generally accepted picture resulting from CASSCF calculations that the excitedstate relaxation of retinal chromophores proceeds via bond inversion and torsional motion around formal double bonds. Differently from previous studies, we employ approaches such as CASPT2 and QMC which are superior to CASSCF as they offer a balanced description of both dynamical and static correlations.

We have also computed the vertical excitations of the retinal models using $\mathrm{CC}$, CASPT2, and QMC and we begin our discussion with a few comments on these results. We find that the $\mathrm{CC}$ and DMC methods give similar excitations for all retinal models and that the CASPT2 excitations are quite sensitive to the internal parameters of the theory. In particular, the excitations computed with the IPEA zero-order Hamiltonian are in close agreement with the $\mathrm{CC}$ and DMC values while resorting to the original CASPT2 formulation lowers the excitations by as much as $0.3 \mathrm{eV}$. The IPEA Hamiltonian was developed to give on average more accurate excitations [79] and its use is here corroborated by the good agreement with other highly-correlated approaches. We also find that the IPEA excitations are more robust as they converge faster with the size of the CAS expansion and are less sensitive to the use of a singleor multi-state approach.

For a comparison with experiments, we consider the 11-cis chromophore where gas phase photodestruction spectroscopy experiments are available [93]. To interpret the complex absorption spectrum of retinal, we follow the recent reassessment of similar experiments on a different chromophore [94] and suggest that the lowestenergy peak corresponds to the adiabatic transition while the vertical lies in the broad shoulder around $2.34 \mathrm{eV}$. Our CASPT2 and DMC vertical excitations computed on the DFT and MP2 ground-state geometries span an energy range of 2.2-2.4 eV which is consistent with this experimental estimate especially given that we did not include vibrational effects which are strong in this system. The excitations computed on the CASSCF geometry are instead significantly higher but can be discarded as our CASPT2 optimizations of planar retinal models show that DFT and MP2 give more accurate geometries than the CASSCF approach.

We discuss now the core of our work and analyze the performance of the various theoretical approaches in describing the excited-state relaxation of retinal models. Our in-plane optimization of the retinal chromophores indicate that the excited-state structures optimized with CASPT2, CC2, CCSD, and VMC agree rather closely while they are at variance with the CASSCF geometries. The CASSCF approach gives strong bond inversion in the excited state, which is not observed when optimizing the structures with the other approaches. According to CASPT2, CC, and VMC, photoexcitation weakens all bonds, which stretch and become partly more 
similar in length while preserving the general bond-length pattern of the ground state. To investigate a non-trivial out-of-plane relaxation, we need to consider a chromophore larger than the model with three double bonds (A or B) since we find that model (B) isomerizes around the central bond both at the CASPT 2 and CASSCF level even though the initial skeletal relaxation proceeds rather differently in the two approaches. Therefore, we investigate the minimal energy path for the out-of-plane motion of model $(\mathrm{C})$ with four double bonds and find that excited-state relaxation at the CASPT2 level proceeds preferentially via a torsional motion around a bond which is formally single in the ground state in agreement with the previous CC calculations by Send and Sundholm [29, 31, 35, 37]. This torsional motion stops at an angle of about $45^{\circ}$ and does not lead to a conical intersection region. On the other hand, in the CASSCF approach, bond inversion is followed by torsion around the cis bond and the molecule is immediately funneled into a conical intersection region from where isomerization can proceed towards the trans product. To investigate the existence of a reactive path at the CASPT2 level, we also consider the constrained excited-state optimization of model (C) around the cis double bond and find a small barrier to isomerization at rather large angles of rotation. Beyond this barrier, the model finally reaches the conical intersection region similarly to the CASSCF approach.

In summary, our CASPT2 results support the picture of a very flexible retinal chromophore in the excited state, where photoexcitation lengthen all bonds so that torsional motion around nearly any bond may contribute to the dynamics. These findings are consistent with recent CC studies [37] which show that retinal models in the excited state have small or vanishing torsional barriers around both formal single and double bonds. This picture must be contrasted to the results of CASSCF calculations which give a stiff chromophore that can only twist around formal double bonds. The flexibility of the excited chromophore in the gas phase observed in CASPT2 and $\mathrm{CC}$ calculations is also compatible with the observation in solution experiments of the existence of multiple minima possibly corresponding to different torsional conformations [67]. Moreover, it has been proposed that the multi-exponential decays observed in solution are related to the possible presence of multiple excited-state paths, some of which are reactive and lead to the photoproduct via the crossing of a conical intersection region while others are non-reactive, do not lead to conical intersection, and are dominant in solution [67]. This interpretation is compatible with our observation of torsional motion around formal single bonds, which is favored starting from the Franck-Condon region, stops at intermediate angles, and does not lead to photoproducts via a conical intersection.

Finally, our results demonstrate the importance of including a balanced description of dynamical and static correlation in the computation of the excited-state gradients. The favorable comparison with the CASPT2 approach indicates that the CC2 method is a useful tool for the study of retinal systems (at least far from the conical intersection region) and that QMC can give accurate gradients when all parameters in the wave function are optimized in energy minimization. Our results raise serious concerns about the common use of the CASSCF approach to investi- 
gate the geometrical relaxation of retinal systems and show that computing singlepoint CASPT2 excitations on CASSCF geometries to partially include the neglected dynamical correlation is generally not a valid procedure to obtain reliable potential energy surfaces. In conclusion, our findings call for a reinvestigation of the photoisomerization mechanism of retinal in the gas phase as well as in the protein environment with higher-level methods than the CASSCF approach.

\subsection{Bibliography}

[1] G. Wald, Science 162, 230 (1968).

[2] T. Okada, M. Sugihara, A.-N. Bondar, M. Elstner, P. Entel, and V. Buss, J. Mol. Biol. 342, 571 (2004).

[3] H. Kandori, Y. Shichida, and T. Yoshizawa, Biochemistry (Moscow) 66, 1483 (2001).

[4] R. W. Schoenlein, L. A. Peteanu, R. A. Mathies, and C. V. Shank, Science 254, 412 (1991).

[5] H. Kandori, Y. Katsuta, M. Ito, and H. Sasabe, J. Am. Chem. Soc. 117, 2669 (1995).

[6] R. S. Becker and K. Freedman, J. Am. Chem. Soc. 107, 1477 (1985).

[7] J. E. Kim, M. J. Tauber, and R. A. Mathies, Biochemistry 40, 13774 (2001).

[8] H. Kandori, Y. Furutani, S. Nishimura, Y. Shichida, H. Chosrowjan, Y. Shibata, and N. Mataga, Chem. Phys. Lett. 334, 271 (2001).

[9] T. Kobayashi, T. Saito, and H. Ohtani, Nature 414, 531 (2001).

[10] J. Herbst, K. Heyne, and R. Diller, Science 297, 822 (2002).

[11] D. W. McCamant, P. Kukura, and R. A. Mathies, J. Phys. Chem. B 109, 10449 (2005).

[12] P. Kukura, D. W. McCamant, S. Yoon, D. B. Wandschneider, and R. A. Mathies, Science 310, 1006 (2005).

[13] P. Kukura, D. W. McCamant, and R. A. Mathies, Annu. Rev. Phys. Chem. 58, 461 (2007).

[14] J. T. Kennis and M.-L. Groot, Curr. Opin. Struct. Biol. 17, 623 (2007).

[15] M. Garavelli, P. Celani, F. Bernardi, M. A. Robb, and M. Olivucci, J. Am. Chem. Soc. 119, 6891 (1997). 
[16] M. Garavelli, T. Vreven, P. Celani, F. Bernardi, M. A. Robb, and M. Olivucci, J. Am. Chem. Soc. 120, 1285 (1998).

[17] M. Garavelli, F. Bernardi, M. Olivucci, T. Vreven, S. Klein, P. Celani, and M. A. Robb, Faraday Discuss. 110, 51 (1998).

[18] R. González-Luque, M. Garavelli, F. Bernardi, M. Merchán, M. A. Robb, and M. Olivucci, Proc. Natl. Acad. Sci. U.S.A. 97, 9379 (2000).

[19] L. De Vico, C. S. Page, M. Garavelli, F. Bernardi, R. Basosi, and M. Olivucci, J. Am. Chem. Soc. 124, 4124 (2002).

[20] C. S. Page and M. Olivucci, J. Comput. Chem. 24, 298 (2003).

[21] M. Wanko, M. Garavelli, F. Bernardi, T. A. Niehaus, T. Frauenheim, and M. Elstner, J. Chem. Phys. 120, 1674 (2004).

[22] S. Fantacci, A. Migani, and M. Olivucci, J. Phys. Chem. A 108, 1208 (2004).

[23] L. Serrano-Andrés, M. Merchán, and R. Lindh, J. Chem. Phys. 122, 104107 (2005).

[24] M. Wanko, M. Hoffmann, P. Strodel, A. Koslowski, W. Thiel, F. Neese, T. Frauenheim, and M. Elstner, J. Phys. Chem. B 109, 3606 (2005).

[25] I. Tavernelli, U. F. Röhrig, and U. Rothlisberger, Mol. Phys. 103, 963 (2005).

[26] F. Blomgren and S. Larsson, J. Comput. Chem. 26, 738 (2005).

[27] A. Cembran, R. Gonzalez-Luque, P. Altoe, M. Merchan, F. Bernardi, M. Olivucci, and M. Garavelli, J. Phys. Chem. A 109, 6597 (2005).

[28] A. J. A. Aquino, M. Barbatti, and H. Lischka, ChemPhysChem 7, 2089 (2006).

[29] R. Send and D. Sundholm, J. Phys. Chem. A 111, 27 (2007).

[30] R. Send and D. Sundholm, Phys. Chem. Chem. Phys. 9, 2862 (2007).

[31] R. Send and D. Sundholm, J. Phys. Chem. A 111, 8766 (2007).

[32] M. Barbatti, G. Granucci, M. Persico, M. Ruckenbauer, M. Vazdar, M. EckertMaksić, and H. Lischka, J. Photochem. Photobiol., A 190, 228 (2007).

[33] A. Cembran, R. González-Luque, L. Serrano-Andrés, M. Merchán, and M. Garavelli, Theor. Chem. Acc. 118, 173 (2007).

[34] O. Weingart, J. Am. Chem. Soc. 129, 10618 (2007).

[35] R. Send and D. Sundholm, J. Mol. Model. 14, 717 (2008). 
[36] J. J. Szymczak, M. Barbatti, and H. Lischka, J. Chem. Theory Comput. 4, 1189 (2008).

[37] R. Send, D. Sundholm, M. P. Johansson, and F. Pawłowski, J. Chem. Theory Comput. 5, 2401 (2009).

[38] I. Schapiro, O. Weingart, and V. Buss, J. Am. Chem. Soc. 131, 16 (2009).

[39] J. J. Szymczak, M. Barbatti, and H. Lischka, J. Phys. Chem. A 113, 11907 (2009).

[40] R. R. Zaari and S. Y. Wong, Chem. Phys. Lett. 469, 224 (2009).

[41] T. Keal, M. Wanko, and W. Thiel, Theor. Chem. Acc. 123, 145 (2009).

[42] A. Warshel, Nature 260, 679 (1976).

[43] A. Warshel and N. Barboy, J. Am. Chem. Soc. 104, 1469 (1982).

[44] R. S. Liu and A. E. Asato, Proc. Natl. Acad. Sci. U.S.A. 82, 259 (1985).

[45] S. Hayashi, E. Tajkhorshid, and K. Schulten, Biophys. J. 85, 1440 (2003).

[46] T. Andruniów, N. Ferré, and M. Olivucci, Proc. Natl. Acad. Sci. U.S.A. 101, 17908 (2004).

[47] U. F. Röhrig, L. Guidoni, A. Laio, I. Frank, and U. Rothlisberger, J. Am. Chem. Soc. 126, 15328 (2004).

[48] U. F. Röhrig, L. Guidoni, and U. Rothlisberger, ChemPhysChem 6, 1836 (2005).

[49] M. Hoffmann, M. Wanko, P. Strodel, P. H. Konig, T. Frauenheim, K. Schulten, W. Thiel, E. Tajkhorshid, and M. Elstner, J. Am. Chem. Soc. 128, 10808 (2006).

[50] P. B. Coto, A. Strambi, N. Ferré, and M. Olivucci, Proc. Natl. Acad. Sci. U.S.A. 103, 17154 (2006).

[51] M. Sugihara, J. Hufen, and V. Buss, Biochemistry 45, 801 (2006).

[52] S. Sekharan, M. Sugihara, and V. Buss, Angew. Chem., Int. Ed. 46, 269 (2007).

[53] K. Bravaya, A. Bochenkova, A. Granovsky, and A. Nemukhin, J. Am. Chem. Soc. 129, 13035 (2007).

[54] K. Fujimoto, S. Hayashi, J.-y. Hasegawa, and H. Nakatsuji, J. Chem. Theory Comput. 3, 605 (2007).

[55] L. M. Frutos, T. Andruniów, F. Santoro, N. Ferré, and M. Olivucci, Proc. Natl. Acad. Sci. U.S.A. 104, 7764 (2007). 
[56] A. Altun, S. Yokoyama, and K. Morokuma, Photochem. Photobiol. 84, 845 (2008).

[57] A. Altun, S. Yokoyama, and K. Morokuma, J. Phys. Chem. B 112, 6814 (2008).

[58] A. Altun, S. Yokoyama, and K. Morokuma, J. Phys. Chem. B 112, 16883 (2008).

[59] A. Altun, S. Yokoyama, and K. Morokuma, J. Phys. Chem. A 113, 11685 (2009).

[60] G. Tomasello, G. Olaso-González, P. Altoeà, M. Stenta, L. Serrano-Andreás, M. Merchaán, G. Orlandi, A. Bottoni, and M. Garavelli, J. Am. Chem. Soc. 131, 5172 (2009).

[61] S. Hayashi, E. Tajkhorshid, and K. Schulten, Biophys. J. 96, 403 (2009).

[62] F. Schautz and C. Filippi, J. Chem. Phys. 120, 10931 (2004).

[63] F. Schautz, F. Buda, and C. Filippi, J. Chem. Phys. 121, 5836 (2004).

[64] F. Cordova, L. J. Doriol, A. Ipatov, M. E. Casida, C. Filippi, and A. Vela, J. Chem. Phys. 127, 164111 (2007).

[65] E. Tapavicza, I. Tavernelli, U. Rothlisberger, C. Filippi, and M. E. Casida, J. Chem. Phys. 129, 124108 (2008).

[66] C. Filippi, M. Zaccheddu, and F. Buda, J. Chem. Theory Comput. 5, 2074 (2009).

[67] G. Zgrablić, S. Haacke, and M. Chergui, J. Phys. Chem. B 113, 4384 (2009).

[68] F. Jensen, in Introduction to Computational Chemistry, 2nd ed. (John Wiley and Sons Ltd: Chichester, U.K., 2007), pp. 133-189.

[69] W. M. C. Foulkes, L. Mitas, R. J. Needs, and G. Rajagopal, Rev. Mod. Phys. 73, 33 (2001).

[70] M. P. Nightingale and V. Melik-Alaverdian, Phys. Rev. Lett. 87, 043401 (2001).

[71] C. J. Umrigar, J. Toulouse, C. Filippi, S. Sorella, and R. G. Hennig, Phys. Rev. Lett. 98, 110201 (2007).

[72] C. Filippi and C. J. Umrigar, Phys. Rev. B 61, R16291 (2000).

[73] C. Attaccalite and S. Sorella, Phys. Rev. Lett. 100, 114501 (2008). 
[74] We find that varying the cutoff parameter $\varepsilon$ between $10^{-2}$ and 1.0 does not lead to appreciable changes in the root-mean-square fluctuations of the VMC forces $(\sigma)$. On the other hand, the use of $\varepsilon=10^{-3}$ and smaller values results in a significant increase in $\sigma$ for the systems studied in this paper.

[75] G. Karlström, R. Lindh, P.-Å. Malmqvist, B. O. Roos, U. Ryde, V. Veryazov, P.-O. Widmark, M. Cossi, B. Schimmelpfennig, P. Neogrady, and L. Seijo, Comput. Mater. Sci. 28, 222 (2003).

[76] A. D. Becke, J. Chem. Phys. 98, 5648 (1993).

[77] Gaussian 03, Revision D.01, Frisch, M. J.; Trucks, G. W.; Schlegel, H. B.; Scuseria, G. E.; Robb, M. A.; Cheeseman, J. R.; Montgomery, Jr., J. A.; Vreven, T.; Kudin, K. N.; Burant, J. C.; Millam, J. M.; Iyengar, S. S.; Tomasi, J.; Barone, V.; Mennucci, B.; Cossi, M.; Scalmani, G.; Rega, N.; Petersson, G. A.; Nakatsuji, H.; Hada, M.; Ehara, M.; Toyota, K.; Fukuda, R.; Hasegawa, J.; Ishida, M.; Nakajima, T.; Honda, Y.; Kitao, O.; Nakai, H.; Klene, M.; Li, X.; Knox, J. E.; Hratchian, H. P.; Cross, J. B.; Bakken, V.; Adamo, C.; Jaramillo, J.; Gomperts, R.; Stratmann, R. E.; Yazyev, O.; Austin, A. J.; Cammi, R.; Pomelli, C.; Ochterski, J. W.; Ayala, P. Y.; Morokuma, K.; Voth, G. A.; Salvador, P.; Dannenberg, J. J.; Zakrzewski, V. G.; Dapprich, S.; Daniels, A. D.; Strain, M. C.; Farkas, O.; Malick, D. K.; Rabuck, A. D.; Raghavachari, K.; Foresman, J. B.; Ortiz, J. V.; Cui, Q.; Baboul, A. G.; Clifford, S.; Cioslowski, J.; Stefanov, B. B.; Liu, G.; Liashenko, A.; Piskorz, P.; Komaromi, I.; Martin, R. L.; Fox, D. J.; Keith, T.; Al-Laham, M. A.; Peng, C. Y.; Nanayakkara, A.; Challacombe, M.; Gill, P. M. W.; Johnson, B.; Chen, W.; Wong, M. W.; Gonzalez, C.; and Pople, J. A.; Gaussian, Inc., Wallingford CT, 2004.

[78] J. Finley, P.-Å. Malmqvist, B. O. Roos, and L. Serrano-Andrés, Chem. Phys. Lett. 288, 299 (1998).

[79] G. Ghigo, B. O. Roos, and P.-Å. Malmqvist, Chem. Phys. Lett. 396, 142 (2004).

[80] B. O. Roos and K. Andersson, Chem. Phys. Lett. 245, 215 (1995).

[81] F. Aquilante, P.-Å. Malmqvist, T. B. Pedersen, A. Ghosh, and B. O. Roos, J. Chem. Theory Comput. 4, 694 (2008).

[82] Aces II, a quantum chemical program package written by J.F. Stanton, J. Gauss, J .D. Watts, P.G. Szalay, R.J. Bartlett with contribution from A.A. Auer, D.B. Bernholdt, O. Christiansen, M.E. Harding, M. Heckert, O. Heun, C. Huber, D. Jonsson, J. Juselius, W.J. Lauderdale, T. Metzroth, K. Ruud and the integral packages MOLECULE (J. Almlof and P.R. Taylor),Props (P.R. Taylor), and ABACUS (T. Helgaker, H.AA. Jensen, P. Jørgensen, and J. Olsen). See also J.F. Stanton, J. Gauss, J. D. Watts, W.J. Lauderdale, R.J. Bartlett, Int. J. Quantum 
Chem. Symp. 26, 879 (1992) as well as : http://www.aces2.de for the current version.

[83] CFOUR, a quantum chemical program package written by J.F. Stanton, J. Gauss, M.E. Harding, P.G. Szalay with contributions from A.A. Auer, R.J. Bartlett, U. Benedikt, C. Berger, D.E. Bernholdt, O. Christiansen, M. Heckert, O. Heun, C. Huber, D. Jonsson, J. Jusélius, K. Klein, W.J. Lauderdale, D. Matthews, T. Metzroth, D.P. O’Neill, D.R. Price, E. Prochnow, K. Ruud, F. Schiffmann, S. Stopkowicz, M.E. Varner, J. Vázquez, F. Wang, J.D. Watts and the integral packages MOLECULE (J. Almlöf and P.R. Taylor), PROPS (P.R. Taylor), ABACUS (T. Helgaker, H.J. Aa. Jensen, P. Jørgensen, and J. Olsen), and ECP routines by A. V. Mitin and C. van Wülle For the current version, see http://www.cfour.de.

[84] CHAMP is a quantum Monte Carlo program package written by C. J. Umrigar, C. Filippi and collaborators.

[85] M. Burkatzki, C. Filippi, and M. Dolg, J. Chem. Phys. 126, 234105 (2007).

[86] C. Filippi and C. J. Umrigar, J. Chem. Phys. 105, 213 (1996), as Jastrow correlation factor, we use the exponential of the sum of three fifth-order polynomials of the electron-nuclear (e-n), the electron-electron (e-e), and of pure 3-body mixed e-e and e-n distances, respectively. The Jastrow factor is adapted to deal with pseudo-atoms, and the scaling factor $\kappa$ is set to 0.60 a.u.

[87] M. W. Schmidt, K. K. Baldridge, J. A. Boatz, S. T. Elbert, M. S. Gordon, J. H. Jensen, S. Koseki, N. Matsunaga, K. A. Nguyen, S. Su, T. L. Windus, M. Dupuis, and J. A. M. Jr, J. Comput. Chem. 14, 1347 (1993).

[88] M. Casula, Phys. Rev. B 74, 161102 (2006).

[89] J. Thom H. Dunning, J. Chem. Phys. 90, 1007 (1989).

[90] P.-O. Widmark, P.-Å. Malmqvist, and B. O. Roos, Theor. Chem. Acc. 77, 291 (1990).

[91] We add one $s$ and one $p$ diffuse function on the carbon and the nitrogen using exponents from the aug-cc-pVDZ basis set, taken from EMSL Basis Set Library (http://bse.pnl.gov).

[92] Supporting information (SI), can be found at http://dx.doi.org/10.1021/ct900692y.

[93] I. B. Nielsen, L. Lammich, and L. H. Andersen, Phys. Rev. Lett. 96, 018304 (2006).

[94] M. W. Forbes and R. A. Jockusch, J. Am. Chem. Soc. 131, 17038 (2009). 
[95] L. De Vico, M. Olivucci, and R. Lindh, J. Chem. Theory Comput. 1, 1029 (2005). 



\section{Chapter 4}

\section{Excitation Energies of Retinal Chromophores: Role of the Structural Model ${ }^{\dagger}$}

We employ a variety of highly-correlated approaches including quantum Monte Carlo (QMC) and the $n$-electron valence state perturbation theory (NEVPT2) to compute the vertical excitation energies of retinal protonated Schiff base (RPSB) models in the gas phase. We find that the NEVPT2 excitation energies are in good agreement with the QMC values and confirm our previous findings that the complete-active-space perturbation (CASPT2) approach yields accurate excitations for RPSB models only when the more recent zero-order IPEA Hamiltonian is employed. The excitations computed with the original zero-order formulation of the CASPT2 approach are instead systematically red-shifted by more than $0.3 \mathrm{eV}$. We then focus on the full 11-cis retinal chromophore and show that the M06-2X and MP2 approaches provide reliable ground-state equilibrium structures for this system while the complete-active-space self-consistent field (CASSCF) geometry is characterized by significantly higher ground-state energies at the NEVPT2 and CASPT2 level. Our calibration of the structural model together with the general agreement of all highly-correlated excited-state methods allows us to reliably assign a value of about $2.3 \mathrm{eV}$ to the vertical excitation of 11-cis RPSB in the gas-phase.

\subsection{Introduction}

The retinal protonated Schiff base (RPSB) chromophore is the light-sensitive molecule present in vertebrate visual opsin proteins, where the interaction with the protein tunes its absorption over a great range of wavelengths from 425 to $560 \mathrm{~nm}$ [1]. To understand the spectral properties of this chromophore and distinguish between

\footnotetext{
${ }^{\dagger}$ This chapter has been published as O. Valsson, C. Angeli, and C. Filippi, "Excitation Energies of Retinal Chromophores: Critical Role of the Structural Model", Phys. Chem. Chem. Phys. 2012, $14,11015-11020$
} 
its intrinsic chemical features and the role of the interaction with the protein environment, the RPSB chromophore has been the subject of extensive theoretical and experimental studies in the gas phase, solution, and protein over the last two decades [1-39]. Many theoretical investigations have focused on the determination of the location of the vertical excitation in the gas phase [20-23,26-28] and, more recently, photo-dissociation experiments have probed the chromophore in the gas phase to assess its absorption properties [17-19].

The theoretical determination of the vertical excitation of RPSB is complicated by the fact that the the estimate depends rather strongly on the methodology employed both to compute the excitation energy and the equilibrium ground-state geometry. Regarded as a gold standard for the computation of excitation energies [40], multi-reference perturbation theory (CASPT2) based on complete-active-space selfconsistent field (CASSCF) wave functions has often been used in retinal studies in combination with CASSCF ground-state equilibrium geometries $[19,20,31,34,41-$ 45]. The two most common choices of zero-order Hamiltonian in CASPT2 [46, 47] have however been recently shown to produce excitation energies of RPSB systems differing by more than $0.3 \mathrm{eV}$ for the same structural model [48]. Our quantum Monte Carlo (QMC) calculations [48] suggest that the more recent zeroorder Hamiltonian, developed to overcome various limitations of the original approach [47], yields superior results, and that the use of the CASSCF method to determine ground-state equilibrium geometries is not adequate for RPSB and should be abandoned in favor of other approaches such as density functional theory with the M06-2X functional or the MP2 approach [48,49]. Finally, the situation is not less confusing on the experimental side as photo-dissociation spectroscopy has produced multiple spectra, sometimes differing for the same RPSB system [18,19]. Currently, the relation between photo-dissociation and optical absorption spectra is in fact the subject of active investigation and debate [49-51].

Here, we revisit the long-debated topic of the vertical excitation energies of RPSB models with the use of the recently introduced $n$-electron valence state perturbation theory (NEVPT2) [52-55]. This modern multi-configuration perturbation approach is also based on CASSCF wave functions but relies on a more advanced zero-order Hamiltonian than CASPT2, where all bielectronic interaction are explicitly included for the active electrons. The similarity between NEVPT2 and CASPT2 in the zero-order wave function and their difference in the zero-order Hamiltonian renders their comparison quite interesting to elucidate how various ingredients affect such perturbative calculations. Importantly, these NEVPT2 calculations also provide an independent assessment of our QMC and other highly-correlated calculations of the vertical excitation energies of RPSB models.

The chapter is organized as follows. In Section 4.2, we describe the computational details and, in Section 4.3, introduce the RPSB models we investigate. We present the results for the vertical excitation energies in Section 4.4 and for the dependence on the choice of structural model in Section 4.5. Finally, in Section 4.6, we discuss our results and conclude. 


\subsection{Computational Details}

We employ here the ANO-L-VDZP basis set [56] with the [3s2p1d] contraction on the $\mathrm{C}$ and $\mathrm{N}$ atoms, and the [2s1p] contraction on the $\mathrm{H}$ atoms. As we have shown in our recent studies $[48,49]$, this basis set yields excitation energies of the minimal RPSB model converged to better than $0.05 \mathrm{eV}$ within both coupled cluster and multireference perturbation theory [48]. For the full 11-cis retinal, this basis gives even smaller basis-set errors since the corresponding excitation is only $0.01 \mathrm{eV}$ higher than the values obtained with augmentation or a larger $[4 \mathrm{~s} 3 \mathrm{p} 1] /[3 \mathrm{~s} 1 \mathrm{p}]$ contraction (see ESI [57]).

We perform the complete active space self-consistent field (CASSCF) calculations with the MOLCAS [58] and the ORCA 2.8 [59-61] codes. In the ORCA calculations for 11-cis retinal, the RIJCOSX approximation [62] for the CASSCF steps and the RI approximation [63] for the integral transformation steps are used. Since a corresponding RI auxiliary basis set is not available for the ANO-L-VDZP basis set, we use the aug-cc-pVTZ auxiliary basis set $[64,65]$. We have adopted and validated this procedure in our recent work on cyanine dyes [66].

The $n$-electron valence state perturbation theory [52-55] (NEVPT2) calculations in both the partially contracted (PC) and the strongly contracted (SC) variants are performed using the ORCA 2.8 code and a stand-alone code interfaced to MOLCAS. The orbital energies for the doubly occupied and virtual orbitals appearing in the Dyall Hamiltonian [67] (used for the definition of the zero-order Hamiltonian in NEVPT2) are obtained by the diagonalization of a generalization of the Fock operator [68] (canonical orbital option in ORCA). For the NEVPT2 calculations with the ORCA code, in the construction of the third- and fourth-order density matrices for 11-cis retinal, the CASSCF wave function is truncated so that only configurations with a weight larger than a threshold of $10^{-10}$ are kept. As shown in the ESI [57], this value for the threshold yields converged results. In the computation of the NEVPT2 excitation energies, we use the same CASSCF wave functions as in our previous CASPT2 study [48]. Specifically, we employ state-average (SA) CASSCF wave functions with equal weights over the two lowest-energy states $\left(S_{0}\right.$ and $\left.S_{1}\right)$. The CAS active space consists of all $\pi$ electrons in the reference and the same number of active $\pi$ orbitals (see ESI [57]).

For the CASPT2 calculations, we use two different zero-order Hamiltonians. One Hamiltonian (0-IPEA) is based on a generalization of the Fock operator for multireference wave functions, which was proposed when the CASPT2 approach was first introduced [46], and has been used in CASPT2 calculations for almost two decades. The second Hamiltonian, named standard IPEA (S-IPEA), is based on a modification (shifting) of the 0-IPEA zero-order Hamiltonian to correct for a systematic error in the original formulation [47], and is the standard option in the latest versions of MOLCAS. We indicate when a constant imaginary shift [69] is used in the CASPT2 calculations to eliminate intruder-state problems.

The CC3 calculations are performed with the Dalton 2.0 program [70]. The CASPT2, QMC, CC2, and CCSD results are from our previous work [48], to which 
we refer the reader for further details. For the DFT geometrical optimizations with the M06-L [71], M06 [72], M06-2X [72], and M06-HF [73] functionals and the cc-pVDZ basis set, we use the Gaussian 09 code [74].

\subsection{Retinal Models}

A

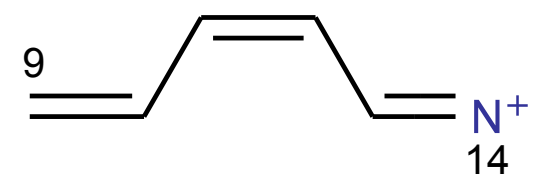

C

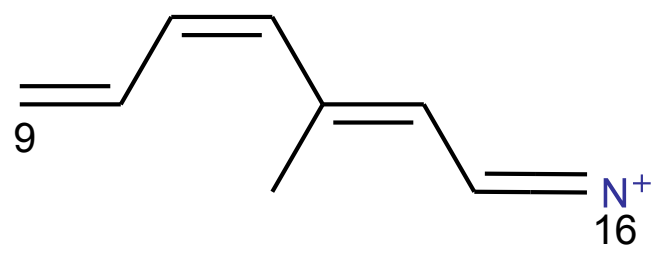

B

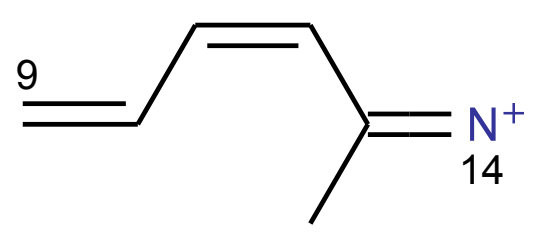

D

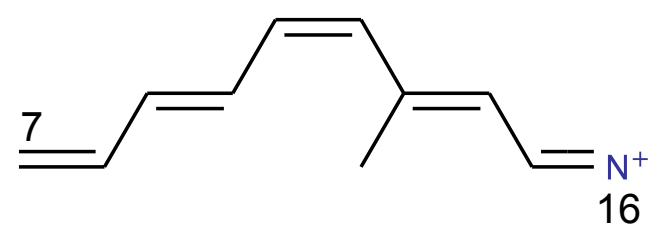

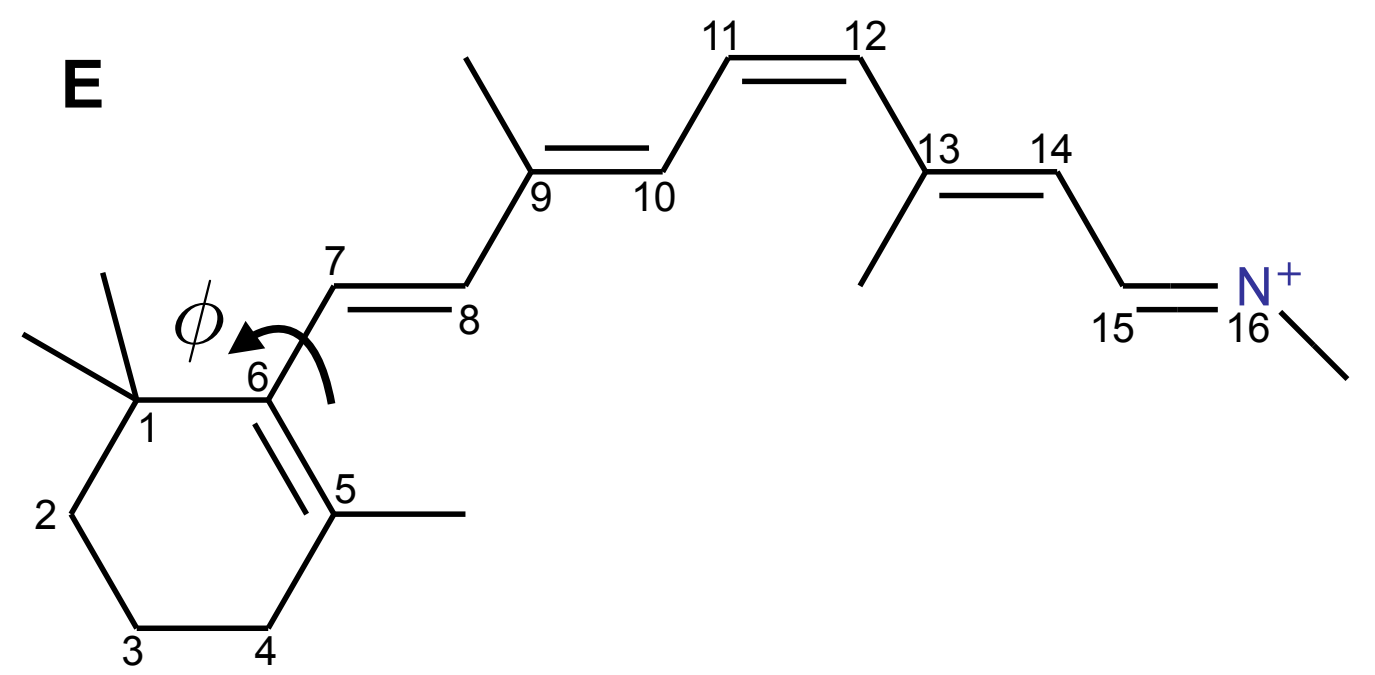

Figure 4.1: Model RPSB chromophores: A) PSB3(0), B) PSB3(1), C) PSB4(1), D) PSB5(1), E) 11-cis chromophore. The naming PSBx(y) denotes the number of double bonds and methyl groups, $\mathrm{x}$ and $\mathrm{y}$, respectively. The atom numbering for chromophore $\mathrm{E}$ is used for all models, so the cis bond is always between $\mathrm{C}_{11}$ and $\mathrm{C}_{12}$. 
The models of RPSB considered in this work are depicted in Figure 4.1. They range from the minimal model (A) to the full 11-cis chromophore (E). For models A to $\mathrm{D}$, we use the naming convention $\operatorname{PSBx}(\mathrm{y})$ where $\mathrm{x}$ and $\mathrm{y}$ indicate the number of double bonds and methyl groups, respectively. For the 11-cis chromophore (E), we consider the $6 \mathrm{~s}-\operatorname{cis}(-)$ orientation of the $\beta$-ionone ring [49], so the $\mathrm{C}_{5}-\mathrm{C}_{6}-\mathrm{C}_{7}-\mathrm{C}_{8}$ dihedral angle of the ring with respect to the conjugated chain (denoted as $\phi$ ) ranges between $-30^{\circ}$ and $-70^{\circ}$ in the various ground-state equilibrium geometries considered here. Unless otherwise stated, the ground-state equilibrium geometries are taken from our previous study [48] and are optimized within DFT with the B3LYP functional and the cc-pVDZ basis set.

\subsection{Vertical Excitation Energies}

In Table 4.1, we report the vertical excitation energies computed with NEVPT2 and CC3 and the ANO-L-VDZP basis, together with the CC2, CCSD, CASPT2, and DMC results we have previously obtained with the same basis set and geometries [48]. We only list the single-state NEVPT2 and CASPT2 excitation energies since the difference with the corresponding quasi-degenerate NEVPT2 and the multi-state CASPT2 values is negligible for models $\mathrm{A}, \mathrm{B}$, and $\mathrm{C}$, and less than $0.06 \mathrm{eV}$ for model D (see ESI [57]).

Table 4.1: Vertical excitation energies (eV) of the RPSB models. The results are obtained employing the ANO-L-VDZP basis set and the ground-state DFT/B3LYP equilibrium geometries. Note that here we present single-state (SS) CASPT2 values while multi-state (MS) CASPT2 values are shown in Table 3.2.

\begin{tabular}{lccccc}
\hline & $\mathrm{A}$ & $\mathrm{B}$ & $\mathrm{C}$ & $\mathrm{D}$ & $\mathrm{E}$ \\
Method & PSB3(0) & PSB3(1) & PSB4(1) & PSB5(1) & 11 -cis \\
\hline SA-CASSCF & 4.56 & 4.80 & 3.74 & 3.10 & 2.51 \\
CASPT2/0-IPEA & 3.74 & 3.85 & 2.99 & 2.50 & $1.85^{a}$ \\
CASPT2/S-IPEA & 4.05 & 4.17 & 3.32 & 2.82 & 2.20 \\
NEVPT2/PC & 4.10 & 4.22 & 3.37 & 2.87 & \\
NEVPT2/SC & 4.17 & 4.28 & 3.43 & 2.92 & 2.26 \\
CC2 & 4.12 & 4.20 & 3.33 & 2.82 & \\
CCSD & 4.23 & 4.37 & 3.47 & 2.95 & \\
CC3 & 4.11 & 4.24 & & & \\
DMC & $4.20(2)$ & $4.42(2)$ & $3.47(2)$ & $3.00(3)$ & $2.37(3)$ \\
\hline
\end{tabular}

${ }^{a}$ Constant imaginary level shift of 0.1 au; 
For all retinal models, it is immediately evident that the CASPT2 approach with the old 0-IPEA Hamiltonian is at variance with all other highly-correlated methods. The CASPT2/0-IPEA excitation energies are systematically red-shifted by more than $0.3 \mathrm{eV}$ with respect to the values obtained with the other approaches. The use of the standard IPEA Hamiltonian (S-IPEA) significantly improves the CASPT2 results and brings them closer to the NEVPT2, CC, and DMC excitation energies. These findings confirm the superiority of the S-IPEA with respect to the 0-IPEA CASPT2 variant, and the presence of a systematic error in the 0-IPEA approach, which had in fact prompted the CASPT2 developers to propose an improved zeroorder Hamiltonian.

Focusing on the multi-reference perturbative approaches, we observe that the NEVPT2 and CASPT2/S-IPEA results are in very good agreement with each other for all retinal models, and within a narrow range of about $0.1 \mathrm{eV}$. The NEVPT2/PC is the higher quality variant of NEVPT2 and gives the best agreement with CASPT2/SIPEA. However, both the SC and PC variants of NEVPT2 yield rather similar excitation energies, which is an indication of the good quality of the zero-order wave functions and the consequent reliability of the results [55,75]. Finally, for all retinal models, the difference between the CASSCF excitation energies and the values obtained with the CASPT2/S-IPEA and NEVPT2 methods is rather small and of the order of $10 \%$. This is also a clear indication of a balanced description of the ground and excited states at zero-order, and shows that the effect of the dynamical correlation on the excitation energies is minor in these systems (with the possible exception of the B model). For all these reasons, the CASPT2/S-IPEA and NEVPT2 results can be considered reliable.

All other highly-correlated approaches are in good agreement with NEVPT2. In particular, $\mathrm{CC} 2, \mathrm{CC} 3$, and CCSD yield rather similar excitations, with the $\mathrm{CC} 2$ values being always red-shifted by about $0.1 \mathrm{eV}$ with respect to CCSD, and very close to $\mathrm{CC} 3$ for models $\mathrm{A}$ and $\mathrm{B}$. For the 11-cis RPSB model, we cannot compute the $\mathrm{CC} 2$ excitation energy with the codes we have available but we can compare to the CC2 results of Ref. 27, where an excitation energy of $2.10 \mathrm{eV}$ on a B3LYP geometry is reported. The difference between this $\mathrm{CC} 2$ value and the corresponding CASPT2 and NEVPT2 excitation energies is therefore larger than for the smaller models, indicating that $\mathrm{CC} 2$ might respond differently to the addition of the $\beta$-ionone ring.

Finally, the excitations computed within diffusion Monte Carlo are slightly blueshifted with respect to the NEVPT2 results and in good agreement with CCSD. The only exception is perhaps the B model, where the discrepancy between CCSD and $\mathrm{DMC}$ on the one side and the multi-reference perturbative approaches on the other is larger than 0.1-0.2 eV. It should be noted that, for model $\mathrm{B}$, the difference between the CASSCF and PT2 excitations is also larger than for all other models $(0.6 \mathrm{eV}$ versus $0.3-0.4 \mathrm{eV}$ ), indicating a more important role of dynamical correlation. For the 11-cis RPSB model, we have here improved the quality of the QMC wave functions with respect to our previous work [48]. In particular, we have further increased the number of configuration state functions (CSF) included in the determinantal component of our Jastrow-Slater wave functions from 10 to 45, by decreasing the threshold 
imposed on the CSF coefficients from 0.08 to 0.04 . The resulting DMC excitation energy is $2.37(3) \mathrm{eV}$, slightly lower but still compatible with the previous value of 2.41(3) eV.

\subsection{Choice of Ground-State Geometry}

Table 4.2: Ground-state and vertical excitation energies of the 11-cis (E) model computed on the DFT, MP2, and CASSCF ground-state equilibrium geometries. For the BLYP, B3LYP, and M06-2X DFT functionals, the percent of exact exchange is reported in parenthesis. The bond length alternation (BLA) and the angle of the $\beta$-ionone ring ( $\phi$, see Figure 4.1) are also listed.

\begin{tabular}{lccccc}
\hline & \multicolumn{5}{c}{ Geometry } \\
\cline { 2 - 6 } & $\begin{array}{c}\text { BLYP } \\
(0 \%)\end{array}$ & $\begin{array}{c}\text { B3LYP } \\
(20 \%)\end{array}$ & $\begin{array}{c}\text { M06-2X } \\
(54 \%)\end{array}$ & MP2 & CASSCF $^{a}$ \\
\hline BLA $(\AA)$ & 0.024 & 0.033 & 0.051 & 0.044 & 0.101 \\
$\phi\left(^{\circ}\right)$ & -29.7 & -33.5 & -38.0 & -40.5 & -68.8 \\
Ground-state energies $(\mathrm{kcal} / \mathrm{mol})$ & & & \\
CASPT2/S-IPEA & +3.60 & +0.69 & 0.00 & -0.40 & +7.01 \\
NEVPT2/SC & +3.99 & +0.44 & 0.00 & +0.60 & +9.05 \\
Vertical excitation energies (eV) & & & \\
SA-CASSCF & 2.36 & 2.51 & 2.71 & 2.64 & 3.25 \\
CASPT2/0-IPEA & $1.77^{b}$ & $1.85^{b}$ & $1.94^{b}$ & $1.89^{b}$ & 2.27 \\
CASPT2/S-IPEA & 2.12 & 2.20 & 2.30 & 2.24 & 2.61 \\
NEVPT2/SC & 2.18 & 2.26 & 2.33 & 2.27 & 2.60 \\
DMC & $2.22(4)^{c}$ & $2.37(3)^{c}$ \\
\hline${ }^{a}$ CASSCF(12,12)/6-31G* geometry from Ref. 20. & & \\
${ }^{b}$ Constant imaginary level shift of 0.1 au. \\
${ }^{c}$ The threshold on the CSFs is 0.04.
\end{tabular}

We now focus on the full 11-cis chromophore and on how the choice of the structural model affects its vertical excitation energy. In Table 4.2, we list the CASPT2, NEVPT2, and DMC excitation energies computed on the ground-state geometries optimized within MP2, CASSCF, and DFT with the BLYP, B3LYP, and M06-2X functionals. We also list the ground-state CASPT2/S-IPEA and NEVPT2 energies together with the most important geometrical parameters [30,49], namely, the bond length alternation (BLA) along the conjugated chain and the angle $\phi$ of the $\beta$-ionone ring. The BLA is here defined as the difference between the averages of the single and double carbon-carbon bonds lengths, and computed including the bonds between $\mathrm{C}_{5}$ and $\mathrm{C}_{15}$. 
To optimize the ground-state geometry within DFT, we employ three functionals with a different amount of exact exchange, namely, BLYP (0\%), B3LYP (20\%), and M06-2X (54\%). This choice of DFT functionals is appropriate for retinal as they span a reasonable range of percentage of exact exchange, one of the parameters in the functional which mainly impacts the geometrical features of this system $[30,49]$. We have recently demonstrated that M06-2X gives a very accurate description of the all-trans RPSB chromophore [49] while larger or smaller amounts of exact exchange lead to structures of inferior quality. As shown in Table 4.2, a similar performance is observed for the 11-cis conformer of RPSB (also see ESI [57] for geometries optimized with other DFT functionals such as M06-HF). The ground-state NEVPT2 energy is the lowest on the M06-2X geometry while CASPT2 gives an energy only $0.4 \mathrm{kcal} / \mathrm{mol}$ higher than the corresponding value on the MP2 geometry. Overall, the B3LYP, M06-2X, and MP2 equilibrium geometries are of comparable good quality since the corresponding ground-state energies are within $1 \mathrm{kcal} / \mathrm{mol}$ of each other both at the NEVPT2 and CASPT2 level. The ground-state geometry optimized with BLYP $(0 \%)$ is instead less optimal.

The CASSCF geometry is significantly different from all other geometries, with bond-length alternation and angle of the $\beta$-ionone ring almost double than the values for the M06-2X geometry. These features of the CASSCF structure have a marked effect on the NEVPT2/SC and CASPT2/S-IPEA ground-state energies, which are more than $7 \mathrm{kcal} / \mathrm{mol}$ higher than the corresponding values computed on the M06$2 \mathrm{X}$ geometry. This confirms our previous findings [49] on the inadequacy of the CASSCF method to describe the geometries of RPSB systems.

As shown in Figure 4.2, the vertical excitation energy of the 11-cis model is very sensitive to the choice of ground-state structure and to the variations in bondlength alternation and angle of the $\beta$-ionone ring as also previously observed in Refs. 23, 26, and 30. In going from the BLYP to the CASSCF structure, both geometrical parameters significantly increase and the corresponding excitation energy grows by as much as $0.4-0.5 \mathrm{eV}$. All highly-correlated approaches display the same trend as a function of the geometry, with CASPT2/S-IPEA and NEVPT2 consistently agreeing within $0.06 \mathrm{eV}$. As observed for all retinal models in the previous section, the CASPT2/0-IPEA excitations are always red-shifted by about $0.3 \mathrm{eV}$.

The M06-2X geometry is characterized by a CASPT2/S-IPEA and a NEVPT2 excitation energy of about $2.3 \mathrm{eV}$ and, together with the B3LYP and MP2 structures, defines a rather narrow range of excitations for the 11-cis model between $2.20 \mathrm{eV}$ and $2.36 \mathrm{eV}$. In striking contrast, the CASSCF equilibrium geometry yields excitation energies close to $2.6 \mathrm{eV}$, that is, more than $0.3 \mathrm{eV}$ blue-shifted with respect to the values at the other geometries. As discussed above, the CASSCF geometry is a less accurate representation of the equilibrium structure of this system and, not surprisingly, is characterized by excitations energies at variance with the ones obtained on more realistic geometries. Finally, it is interesting to note that the CASPT2/0-IPEA excitation energy on the CASSCF geometry is very close to the CASPT2/S-IPEA value at the optimal M06-2X geometry. In the past, this coincidental agreement has masked the inadequacy of the use of CASSCF geometries for retinal. More gener- 


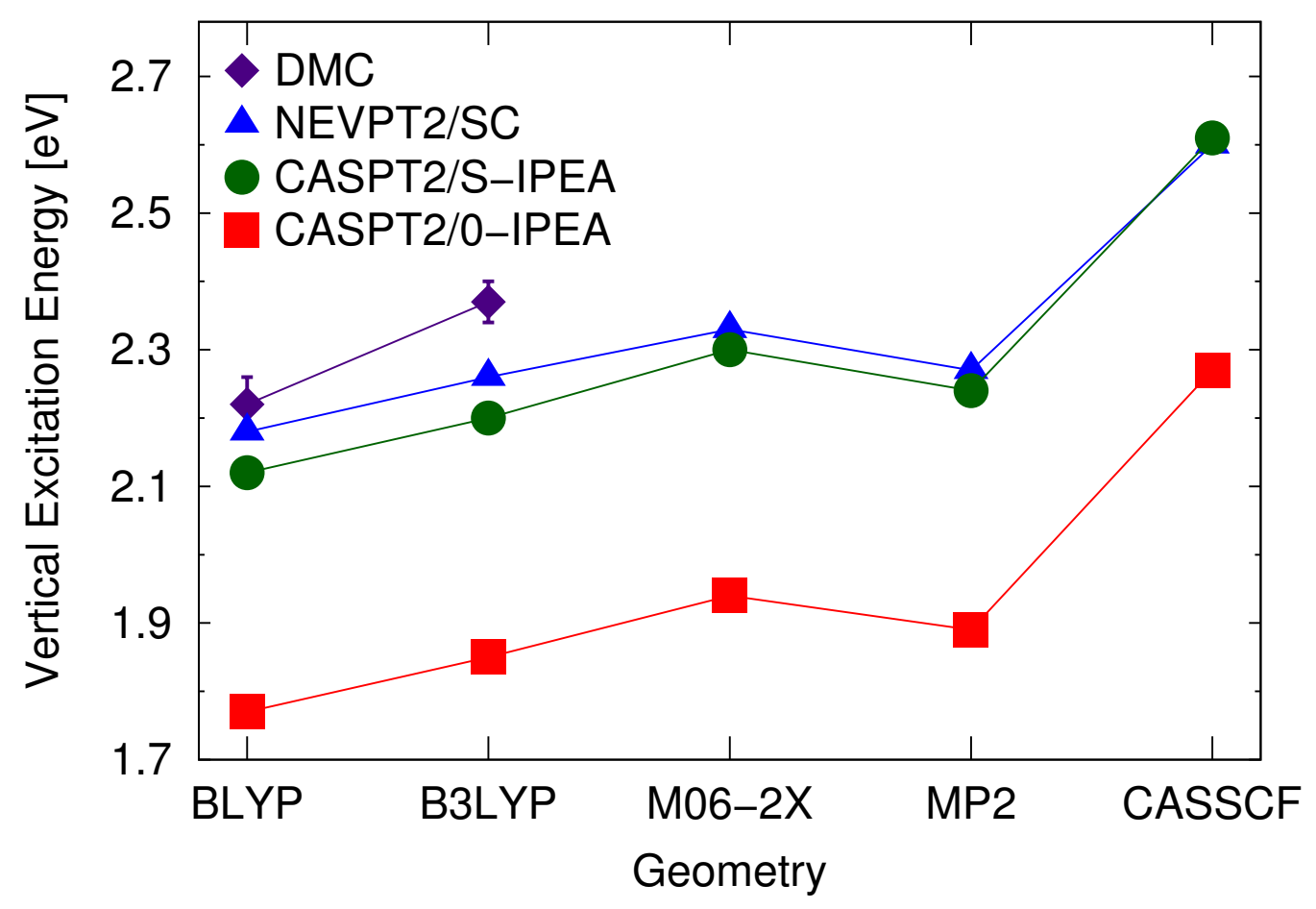

Figure 4.2: Vertical excitation energies (eV) of the 11-cis retinal (E) model computed on the DFT, MP2, and CASSCF ground-state equilibrium geometries.

ally, the use of the CASSCF approach for the calculation of ground-state structures is often coupled to the use of the 0-IPEA zero-order Hamiltonian for the calculation of the CASPT2 excitations, which results in a fortuitous cancellation of errors [48]. Nevertheless, we emphasize again that the results obtained here with a variety of highly-correlated methods clearly indicate that the 0-IPEA approach severely underestimates the excitation energies of retinal and should therefore not be used.

Finally, we note that, for the all-trans RPSB chromophore characterized by a similar orientation of the $\beta$-ionone ring, we have obtained CASPT2/S-IPEA and NEVPT2 excitation energies of $2.31 \mathrm{eV}$ and $2.34 \mathrm{eV}$, respectively, when using a M06-2X geometry [49]. These values are very close to the corresponding excitation energies of the 11-cis RPSB, demonstrating that the difference between the two isomers does not affect the excitation energies.

\subsection{Discussion and Conclusions}

We have here extended our previous theoretical study of RPSB models [48] to clarify several important issues, which have been the subject of a lingering debate. To this aim, we have employed the NEVPT2 method which is a modern multi-configuration perturbation approach based on CASSCF wave functions. NEVPT2 relies on a more 
advanced zero-order Hamiltonian than CASPT2 (all bielectronic interaction among the active electrons are explicitly taken into account), which makes NEVPT2 free from some of the problems affecting CASPT2 as the possible appearance of intruder states [55]. Although based on different zero-order Hamiltonians, NEVPT2 and CASPT2 share the same methodological framework so that NEVPT2 can serve as an interesting and yet independent test of the results previously obtained by our group [48, 49] and other authors [20-23, 26, 28]. For example, it can help us to understand which zero-order Hamiltonian, S-IPEA or 0-IPEA, should be used in the CASPT2 calculations and further validate the findings obtained with other highlycorrelated approaches such as CC or QMC.

For all retinal models, we find that NEVPT2 yields excitation energies rather close to the values computed with the CASPT2/S-IPEA, CC, and DMC methods. The CASPT2/0-IPEA excitation energies are instead systematically red-shifted by more than $0.3 \mathrm{eV}$ with respects to all other results. The overall agreement of the NEVPT2, DMC, and CC methods gives us confidence on the quality of our estimates for the excitation energies of retinal models. Furthermore, our findings support the conclusion that the use of the S-IPEA zero-order Hamiltonian in CASPT2 is superior to the older 0-IPEA variant, which has been so often employed in retinal studies over many years $[19,20,31,34,41-45]$.

To assess the impact of the choice of ground-state equilibrium geometry on the excitation energy, we then focus on the 11-cis retinal. We find that the M06-2X and MP2 methods are reliable approaches to compute the equilibrium structure of this system while the use of the CASSCF method yields a structure characterized by much higher ground-state CASPT2/S-IPEA and NEVPT2 total energies. Furthermore, the geometrical parameters of the CASSCF model (i.e. BLA and the orientation of the $\beta$-ionone ring) are significantly different from those of the accurate M06-2X geometry. Importantly, these features of the CASSCF geometry strongly affect the excitation energy, which is blue-shifted by as much as $0.3 \mathrm{eV}$ with respect to what is obtained on the better M06-2X structure. The use of CASSCF geometries for retinal systems is quite widespread in studies on the retinal molecule $[19,20,31,34,41-45]$ and has been generally combined with the CASPT2/0-IPEA method for the calculation of the excitation energies. This combination leads to an incorrect description of the system, which is however masked by a fortuitous cancellation of errors in the estimate of the vertical excitation energy (the blue shift due to the use of a CASSCF equilibrium geometry is mostly compensated by an erroneous red shift of the older 0-IPEA variant of CASPT2).

Finally, our calculations with a variety of highly-correlated approaches on reliable ground-state equilibrium geometries define a range of 2.20-2.37 eV for the vertical excitation energy of the 11-cis model, with NEVPT2 theory yielding a value of about $2.3 \mathrm{eV}$ on the optimal M06-2X structure. This estimate should be preferably confirmed by experimental findings but a comparison with available experiments is unfortunately problematic. Even though the retinal chromophore has been extensively studied in absorption experiments in solution [2,9,14], it is difficult to infer an estimate of the gas-phase vertical excitation from these experiments. The absorption 
spectrum in solution is highly dependent on the particular solvent, and counter-ions induce significant blue shifts in the absorption spectrum since they strongly influence the extended $\pi$-electron system of retinal. For instance, the absorption maximum of all-trans RPSB in 20 different solvents [2] in the presence of $\mathrm{CHCl}_{2} \mathrm{CO}_{2}^{-}$ counter-ions ranges between 2.64 and $2.93 \mathrm{eV}$, and is therefore significantly higher than the expected vertical excitation in the gas phase. Gas-phase photo-dissociation experiments should instead allow a direct comparison with our and other theoretical calculations. However, such comparison is also not straightforward since the relation between photo-dissociation and optical absorption spectra is not simple and is in fact the subject of active investigation and debate [49-51]. For RPSB, early gas-phase photo-dissociation experiments on the 11-cis chromophore [18] produced a spectrum with a strong band at $2.03 \mathrm{eV}$ and a broad shoulder of slightly lower intensity, which extends up to $2.34 \mathrm{eV}$. However, if we consider the experimental evidence on the all-trans retinal, which we find to have the same excitation energy as the 11-cis conformer, there are two rather different photo-dissociation spectra produced by the same group, one with a main band at $2.00 \mathrm{eV}$ and at least two additional shoulders [18], and the other with a practically flat plateau between $2.03 \mathrm{eV}$ and $2.34 \mathrm{eV}$ [19]. Here, we will not discuss the interpretation and potential complications of photo-dissociation experiments but only stress that the convergence of our highly-correlated CASPT2, NEVPT2, and DMC excited-state calculations together with our careful calibration of the equilibrium geometry gives us confidence in assigning the vertical excitation of the 11-cis RPSB at about $2.3 \mathrm{eV}$.

\subsection{Bibliography}

[1] T. Ebrey and Y. Koutalos, Prog. Retinal Eye Res. 20, 49 (2001).

[2] H. Houjou, Y. Inoue, and M. Sakurai, J. Am. Chem. Soc. 120, 4459 (1998).

[3] G. G. Kochendoerfer, S. W. Lin, T. P. Sakmar, and R. A. Mathies, Trend. Biochem. Sci. 24, 300 (1999).

[4] J. Nathans, Neuron 24, 299 (1999).

[5] S. Yokoyama, Prog. Retinal Eye Res. 19, 385 (2000).

[6] S. T. Menon, M. Han, and T. P. Sakmar, Physiol. Rev. 81, 1659 (2001).

[7] R. Stenkamp, S. Filipek, C. Driessen, D. Teller, and K. Palczewski, Biochim. Biophys. Acta 1565, 168 (2002).

[8] R. E. Stenkamp, D. C. Teller, and K. Palczewski, Arch. Pharm. Pharm. Med. Chem. 338, 209 (2005).

[9] G. Zgrablić, K. Voitchovsky, M. Kindermann, S. Haacke, and M. Chergui, Biophys. J. 88, 2779 (2005). 
[10] K. Palczewski, Annu. Rev. Biochem. 75, 743 (2006).

[11] B. Nickle and P. R. Robinson, Cell. Mol. Life. Sci. 64, 2917 (2007).

[12] J. K. Bowmaker, Vision Res. 48, 2022 (2008).

[13] M. B. Nielsen, Chem. Soc. Rev. 38, 913 (2009).

[14] G. Zgrablić, S. Haacke, and M. Chergui, J. Phys. Chem. B 113, 4384 (2009).

[15] K. Tsutsui and Y. Shichida, Photochem. Photobiol. Sci. 9, 1426 (2010).

[16] S. O. Smith, Annu. Rev. Biophys. 39, 309 (2010).

[17] L. H. Andersen, I. B. Nielsen, M. B. Kristensen, M. O. A. El Ghazaly, S. Haacke, M. B. Nielsen, and M. A.. Petersen, J. Am. Chem. Soc. 127, 12347 (2005).

[18] I. B. Nielsen, L. Lammich, and L. H. Andersen, Phys. Rev. Lett. 96, 018304 (2006).

[19] J. Rajput, D. Rahbek, L. Andersen, A. Hirshfeld, M. Sheves, P. Altoè, G. Orlandi, and M. Garavelli, Angew. Chem. Int. Ed. 49, 1790 (2010).

[20] A. Cembran, R. Gonzalez-Luque, P. Altoe, M. Merchan, F. Bernardi, M. Olivucci, and M. Garavelli, J. Phys. Chem. A 109, 6597 (2005).

[21] S. Sekharan, O. Weingart, and V. Buss, Biophys. J. 91, L07 (2006).

[22] K. Bravaya, A. Bochenkova, A. Granovsky, and A. Nemukhin, J. Am. Chem. Soc. 129, 13035 (2007).

[23] R. Send and D. Sundholm, Phys. Chem. Chem. Phys. 9, 2862 (2007).

[24] R. Send and D. Sundholm, J. Phys. Chem. A 111, 27 (2007).

[25] R. Send and D. Sundholm, J. Phys. Chem. A 111, 8766 (2007).

[26] A. Altun, S. Yokoyama, and K. Morokuma, J. Phys. Chem. B 112, 16883 (2008).

[27] R. R. Zaari and S. Y. Wong, Chem. Phys. Lett. 469, 224 (2009).

[28] R. Send, V. R. I. Kaila, and D. Sundholm, J. Chem. Theory Comput. 7, 2473 (2011).

[29] U. F. Röhrig, L. Guidoni, and U. Rothlisberger, ChemPhysChem 6, 1836 (2005).

[30] M. Wanko, M. Hoffmann, P. Strodel, A. Koslowski, W. Thiel, F. Neese, T. Frauenheim, and M. Elstner, J. Phys. Chem. B 109, 3606 (2005). 
[31] P. B. Coto, A. Strambi, N. Ferré, and M. Olivucci, Proc. Natl. Acad. Sci. U.S.A. 103, 17154 (2006).

[32] M. Hoffmann, M. Wanko, P. Strodel, P. H. König, T. Frauenheim, K. Schulten, W. Thiel, E. Tajkhorshid, and M. Elstner, J. Am. Chem. Soc. 128, 10808 (2006).

[33] K. Fujimoto, J.-Y. Hasegawa, and H. Nakatsuji, Chem. Phys. Lett. 462, 318 (2008).

[34] G. Tomasello, G. Olaso-González, P. Altoeà, M. Stenta, L. Serrano-Andreás, M. Merchaán, G. Orlandi, A. Bottoni, and M. Garavelli, J. Am. Chem. Soc. 131, 5172 (2009).

[35] H. C. Watanabe, Y. Mori, T. Tada, S. Yokoyama, and T. Yamato, BIOPHYSICS 6, 67 (2010).

[36] R. Rajamani, Y.-L. Lin, and J. Gao, J. Comput. Chem. 32, 854 (2011).

[37] S. Sekharan and K. Morokuma, J. Am. Chem. Soc. 133, 19052 (2011).

[38] J. S. Frähmcke, M. Wanko, and M. Elstner, J. Phys. Chem. B 116, 3313 (2012).

[39] V. R. I. Kaila, R. Send, and D. Sundholm, J. Phys. Chem. B 116, 2249 (2012).

[40] D. Roca-Sanjuán, F. Aquilante, and R. Lindh, WIREs Comput Mol Sc 2, 585 (2012).

[41] M. Garavelli, P. Celani, F. Bernardi, M. A. Robb, and M. Olivucci, J. Am. Chem. Soc. 119, 6891 (1997).

[42] R. González-Luque, M. Garavelli, F. Bernardi, M. Merchán, M. A. Robb, and M. Olivucci, Proc. Natl. Acad. Sci. U.S.A. 97, 9379 (2000).

[43] T. Andruniów, N. Ferré, and M. Olivucci, Proc. Natl. Acad. Sci. U.S.A. 101, 17908 (2004).

[44] A. Cembran, R. González-Luque, L. Serrano-Andrés, M. Merchán, and M. Garavelli, Theor. Chem. Acc. 118, 173 (2007).

[45] I. Schapiro, M. N. Ryazantsev, L. M. Frutos, N. Ferré, R. Lindh, and M. Olivucci, J. Am. Chem. Soc. 133, 3354 (2011).

[46] K. Andersson, P.-A. Malmqvist, B. O. Roos, A. J. Sadlej, and K. Wolinski, J. Phys. Chem. 94, 5483 (1990).

[47] G. Ghigo, B. O. Roos, and P.-Å. Malmqvist, Chem. Phys. Lett. 396, 142 (2004).

[48] O. Valsson and C. Filippi, J. Chem. Theory Comput. 6, 1275 (2010). 
[49] O. Valsson and C. Filippi, J. Phys. Chem. Lett. 3, 908 (2012).

[50] K. Chingin, R. M. Balabin, V. Frankevich, K. Barylyuk, R. Nieckarz, P. Sagulenko, and R. Zenobi, Int. J. Mass Spectrom. 306, 241 (2011).

[51] M. W. Forbes, A. M. Nagy, and R. A. Jockusch, Int. J. Mass Spectrom. 308, 155 (2011).

[52] C. Angeli, R. Cimiraglia, S. Evangelisti, T. Leininger, and J.-P. Malrieu, J. Chem. Phys. 114, 10252 (2001).

[53] C. Angeli, R. Cimiraglia, and J.-P. Malrieu, Chem. Phys. Lett. 350, 297 (2001).

[54] C. Angeli, R. Cimiraglia, and J.-P. Malrieu, J. Chem. Phys. 117, 9138 (2002).

[55] C. Angeli, M. Pastore, and R. Cimiraglia, Theor. Chem. Acc. 117, 743 (2007).

[56] P. Widmark, P. Malmqvist, and B. O. Roos, Theor. Chem. Acc. 77, 291 (1990).

[57] Electronic supplementary information (ESI), can be found at http://dx.doi.org/10.1039/c2cp41387f.

[58] F. Aquilante, L. De Vico, N. Ferré, G. Ghigo, P.-A. Malmqvist, P. Neogrády, T. B. Pedersen, M. Pitoňák, M. Reiher, B. O. Roos, L. Serrano-Andrés, M. Urban, V. Veryazov, and R. Lindh, J. Comput. Chem. 31, 224 (2010).

[59] Neese, F. ORCA - an ab initio, Density Functional and Semiempirical program package, Version 2.8. Max-Planck-Insitut für Bioanorganische Chemie, Mülheim an der Ruhr, 2011.

[60] F. Neese, WIREs Comput Mol Sc 2, 73 (2012).

[61] F. Neese, D. G. Liakos, and S. Ye, J. Biol. Inorg. Chem. 16, 821 (2011).

[62] F. Neese, F. Wennmohs, A. Hansen, and U. Becker, Chem. Phys. 356, 98 (2009).

[63] K. Eichkorn, O. Treutler, H. Öhm, M. Häser, and R. Ahlrichs, Chem. Phys. Lett. 240, 283 (1995).

[64] T. H. Dunning Jr, J. Chem. Phys. 90, 1007 (1989).

[65] F. Weigend, A. Köhn, and C. Hättig, J. Chem. Phys. 116, 3175 (2002).

[66] R. Send, O. Valsson, and C. Filippi, J. Chem. Theory Comput. 7, 444 (2011).

[67] K. G. Dyall, J. Chem. Phys. 102, 4909 (1995).

[68] C. Angeli, R. Cimiraglia, and J.-P. Malrieu, Chem. Phys. Lett. 317, 472 (2000).

[69] N. Forsberg and P.-Å. Malmqvist, Chem. Phys. Lett. 274, 196 (1997). 
[70] DALTON, an ab initio electronic structure program, release 2.0, see http://www.kjemi.uio.no/software/dalton/dalton.html, 2005.

[71] Y. Zhao and D. G. Truhlar, J. Chem. Phys. 125, 194101 (2006).

[72] Y. Zhao and D. G. Truhlar, Theor. Chem. Acc. 120, 215 (2008).

[73] Y. Zhao and D. G. Truhlar, J. Phys. Chem. A 110, 13126 (2006).

[74] M. J. Frisch, G. W. Trucks, H. B. Schlegel, G. E. Scuseria, M. A. Robb, J. R. Cheeseman, G. Scalmani, V. Barone, B. Mennucci, G. A. Petersson, H. Nakatsuji, M. Caricato, X. Li, H. P. Hratchian, A. F. Izmaylov, J. Bloino, G. Zheng, J. L. Sonnenberg, M. Hada, M. Ehara, K. Toyota, R. Fukuda, J. Hasegawa, M. Ishida, T. Nakajima, Y. Honda, O. Kitao, H. Nakai, T. Vreven, J. A. Montgomery, Jr., J. E. Peralta, F. Ogliaro, M. Bearpark, J. J. Heyd, E. Brothers, K. N. Kudin, V. N. Staroverov, R. Kobayashi, J. Normand, K. Raghavachari, A. Rendell, J. C. Burant, S. S. Iyengar, J. Tomasi, M. Cossi, N. Rega, J. M. Millam, M. Klene, J. E. Knox, J. B. Cross, V. Bakken, C. Adamo, J. Jaramillo, R. Gomperts, R. E. Stratmann, O. Yazyev, A. J. Austin, R. Cammi, C. Pomelli, J. W. Ochterski, R. L. Martin, K. Morokuma, V. G. Zakrzewski, G. A. Voth, P. Salvador, J. J. Dannenberg, S. Dapprich, A. D. Daniels, Ö. Farkas, J. B. Foresman, J. V. Ortiz, J. Cioslowski, and D. J. Fox, Gaussian 09 Revision A.02, Gaussian Inc. Wallingford CT 2009.

[75] C. Angeli and M. Pastore, J. Chem. Phys. 134, 184302 (2011). 



\section{Chapter 5}

\section{Gas-Phase Retinal Spectroscopy: Temperature Effects Are But a Mirage $^{\dagger}$}

We employ state-of-the-art first-principle approaches to investigate whether temperature effects are responsible for the unusually broad and flat spectrum of protonated Schiff base retinal observed in photo-dissociation spectroscopy, as has recently been proposed. We first carefully calibrate how to construct a realistic geometrical model of retinal, and show that the exchange-correlation M06-2X functional yields an accurate description while the commonly used complete-active-space self-consistentfield method (CASSCF) is not adequate. Using modern multiconfigurational perturbative methods (NEVPT2) to compute the excitations, we then demonstrate that conformations with different orientations of the $\beta$-ionone ring are characterized by similar excitations. Moreover, other degrees of freedom identified as active in roomtemperature molecular dynamics simulations do not yield the shift required to explain the anomalous spectral shape. Our findings indicate that photo-dissociation experiments are not representative of the optical spectrum of retinal in the gas phase and call for further experimental characterization of the dissociation spectra.

\subsection{Introduction}

The retinal protonated Schiff base (RPSB) chromophore represents a fascinating archetype of a photosensitive biological component since it functions as light detector over a remarkably wide range of absorption energies in visual [1,2] $(425-560 \mathrm{~nm})$ and archaeal [3] (480-590 nm) rhodopsins. To understand how the protein tunes absorption over so many wave-lengths, it is important to establish the spectral behavior of retinal in the gas phase to discern intrinsic geometric and electronic features from the response of the chromophore to the biological microenvironment. Photo-

\footnotetext{
${ }^{\dagger}$ This chapter has been published as O. Valsson and C. Filippi, "Gas-Phase Retinal Spectroscopy: Temperature Effects Are But a Mirage”, J. Phys. Chem. Lett. 2012, 3, 908-912
} 
induced dissociation spectroscopy $[4,5]$ represents in principle an ideal experimental technique for this purpose as the measurement can probe charged molecules in the gas phase at low target densities in the absence of external perturbations. For alltrans RPSB, early dissociation spectroscopy experiments [4] produced an absorption spectrum with a strong band at $600 \mathrm{~nm}$ and at least two additional shoulders (Figure 5.1a), while a more recent experiment by the same group [5] has obtained a spectrum with a practically flat plateau between 530 and $610 \mathrm{~nm}$ (Figure 5.1b). These observations have separately received a high level of attention and have motivated many theoretical attempts to quantitatively reproduce them [5-12]. Unfortunately, the difference between the two spectra was not well rationalized experimentally nor further characterized, for instance by intensity dependence studies. This is particularly relevant since retinal is not the only chromophore for which multiple distinct dissociation spectra have been produced depending on experimental factors such as excitation laser power [13-16]. In measurements for the green fluorescent protein chromophore, it has in fact recently been argued that the dissociation spectrum does not reliably represent the optical spectrum of this system $[15,16]$.

The unusually flat profile of the dissociation spectrum was explained in terms of temperature effects [5]. The RPSB chromophore could easily isomerize between conformers with different orientations of the $\beta$-ionone ring with respect to the plane of the conjugate chain (Figure 5.2). If these configurations are characterized by excitations spanning the appropriate range, temperature fluctuations could be responsible for the observed experimental plateau in the spectrum. The interpretation of the dissociation spectrum of RPSB in terms of temperature effects was however put forward by modeling the conformer structures with the complete-active-space selfconsistent-field [17] (CASSCF) method, which largely lacks inclusion of dynamical correlation. This theoretical study cannot therefore be considered conclusive. In fact, calculations employing a very different framework based on density functional theory (DFT) indicate that temperature fluctuations might not significantly affect absorption [18]. Consequently, the role of temperature effects on the gas-phase spectroscopy of RPSB remains an open issue due to uncertainties both on the experimental and theoretical sides. Importantly, visual and archaeal rhodopsins differ in their retinal conformers [3] and it is therefore relevant to assess whether internal variations in the structure or the interaction with the protein pocket are responsible for shifts in the spectrum.

Here, we present a careful investigation of the impact of conformational variations of the chromophore on its absorption properties. Importantly, we do not assume that a given theoretical technique gives us the correct answer but carefully calibrate the approach employed to construct the structural model of the chromophore in the ground state. This is a key point in our work since the role of an appropriate choice of structures has been often neglected or largely overlooked in previous studies. After we identify which structural models are representative of retinal in the gas phase, we then analyze how to accurately compute the excitation energies of the multiple conformers to elucidate the structural dependence of absorption in RPSB. 
a) Ref. 4

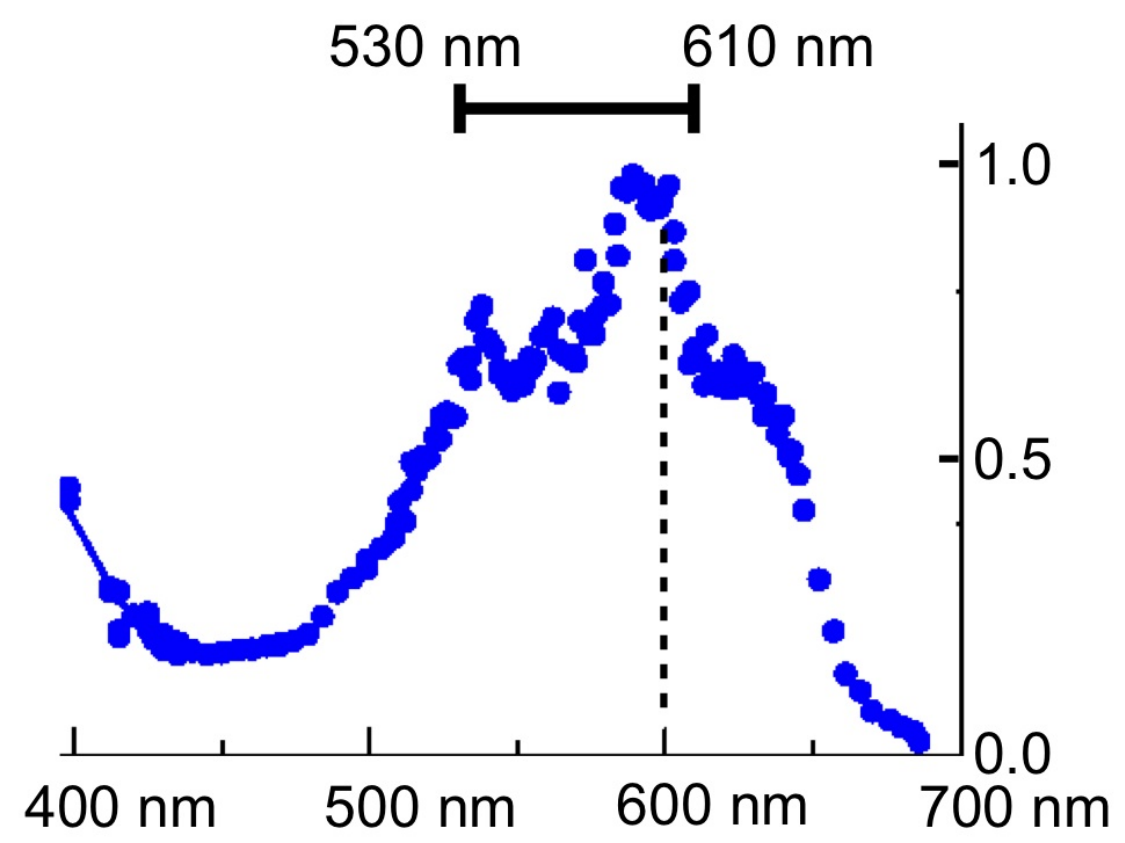

b) Ref. 5

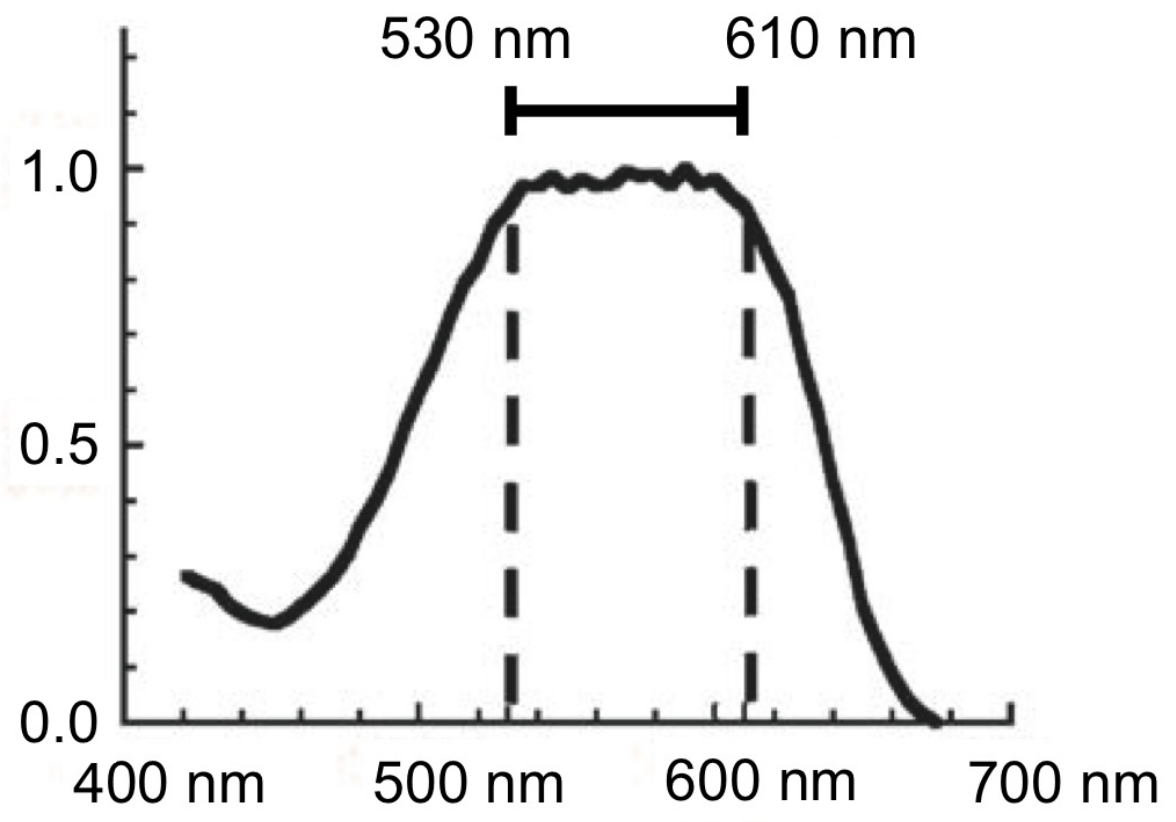

Figure 5.1: The absorption spectra obtained for all-trans RPSB in dissociation spectroscopy experiments from a) Ref. 4 in 2006, and b) Ref. 5 in 2010. 


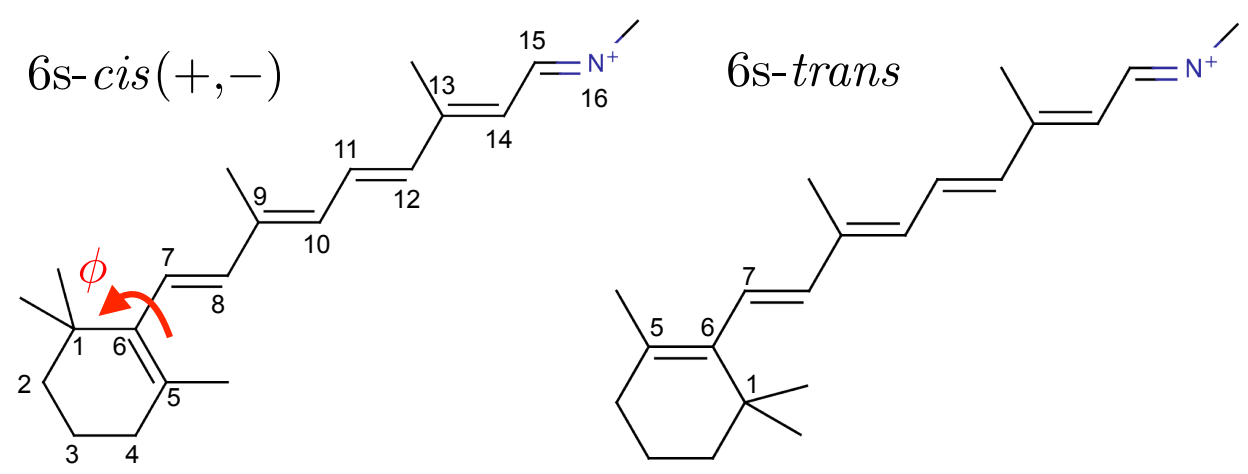

Figure 5.2: 6s-cis and 6s-trans conformers of RPSB.

\subsection{Results}

We first investigate the choice of theoretical method to construct the conformers for the 6s-trans, 6s-cis(+), and 6s-cis(-) orientations of the $\beta$-ionone ring (Figure 5.2), and to estimate the height of the barrier between them. We employ a selected set of modern DFT functionals characterized by a different percentage of exact exchange, namely, BLYP [19,20] (0\%), B3LYP [21,22] (20\%), M06-2X [23] (54\%), and M06HF [23] (100\%). This choice of exchange-correlation approximations is particularly appropriate for retinal since it spans a significant range of percentages of exact exchange, which is one of the most important parameters influencing the structural properties of this chromophore [24] (see also SI [25]).

For each functional, we perform a series of geometrical optimizations at constrained angles of the $\beta$-ionone ring, $\phi$ (Figure 5.2), while relaxing all other degrees of freedom. As shown in Figure 5.3a, the resulting potential energy curves are rather different in particular as regards the height of the barrier between trans and cis, which decreases when we increase the amount of exact exchange. The relative stability of the conformers also varies with trans/cis being favored at low/high exchange. Finally, several structural features of the minima are also affected, most notably, the twist angle of the $\beta$-ionone ring in the cis orientations and the bond length alternation, both significantly increasing for higher amounts of exchange (see SI [25]).

To establish which structures most reliably describe retinal, we compute the ground-state energies of all geometries with an accurate highly correlated approach, namely, the complete-active-space second-order perturbation theory (CASPT2) [27, 28]. As shown in Figure 5.3b, the M06-2X functional with its intermediate amount of exact exchange leads to the most accurate description of the geometrical features of retinal. The CASPT2 energies computed on the M06-2X structures are in fact the lowest while the use of either lower or higher percentages of exact exchange results in higher ground-state energies. The CASSCF structures display instead the worst performance, yielding the highest CASPT2 ground-state energies. Therefore, 


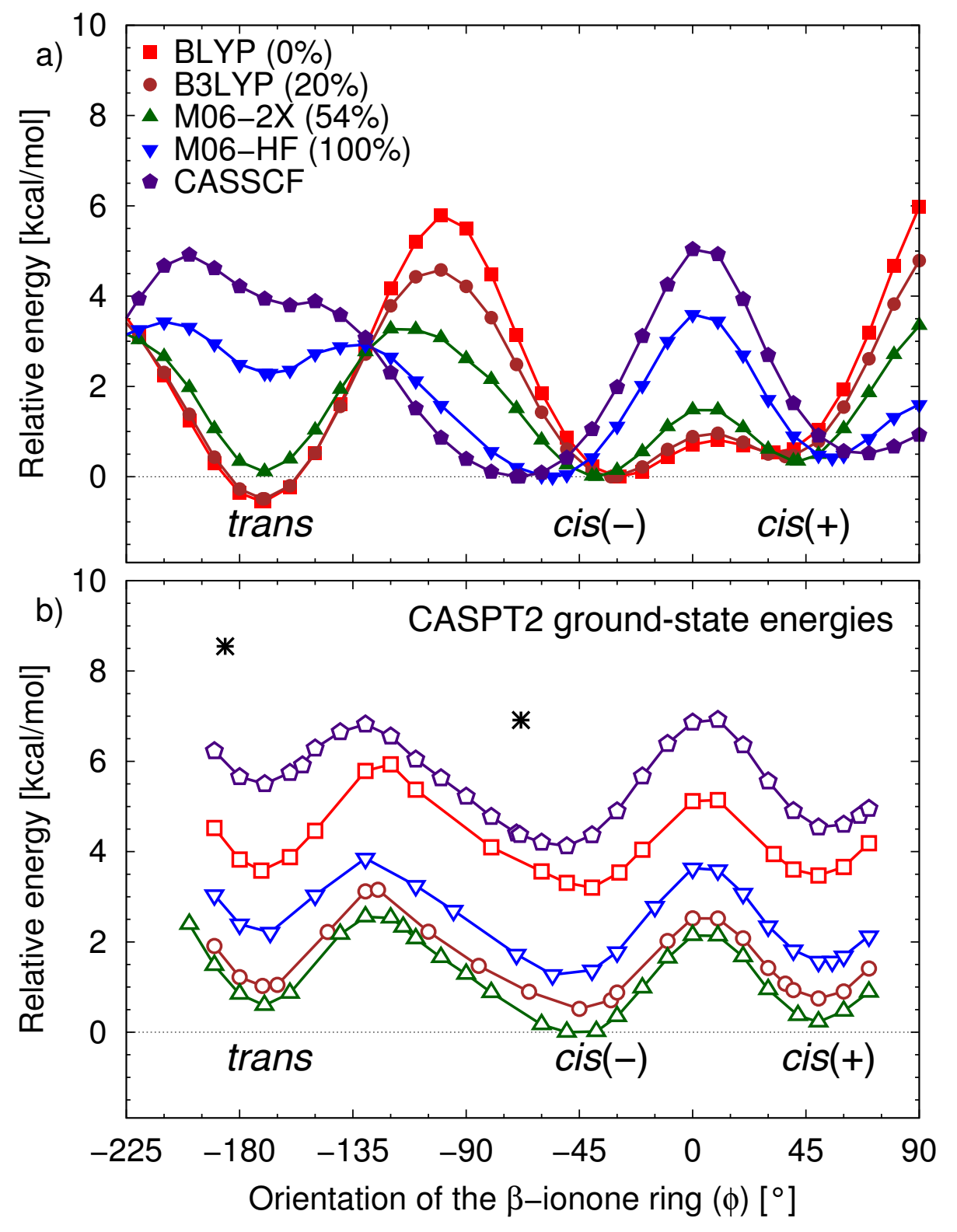

Figure 5.3: (a) DFT and CASSCF potential energy curves as a function of the orientation of the $\beta$-ionone ring, computed with different functionals (full symbols). The percentage of exact exchange is given in parenthesis. (b) CASPT2 ground-state energies computed on the DFT and CASSCF ground-state geometries (empty symbols). We also evaluate the CASPT2 energies on the CASSCF ground-state geometries reported in Ref. 5 (star symbols) [26]. 
CASSCF geometries are not appropriate to describe gas-phase retinal despite having being widely employed for numerous retinal studies in the last decade. It is also important to stress that the interpretation of the broad plateau in the dissociation spectrum in terms of temperature effects was in fact based on the use of CASSCF structural models [5]. For completeness, we compute accurate ground-state energies precisely on the two geometries previously employed to explain the spectrum [5], and obtain the two highest energy points [26] in Figure 5.3b.

Having established the reliability of M06-2X to describe the structural features of retinal, we are now in a position to assess whether the presence of different isomers might explain the flat plateau in the photo-induced dissociation spectrum. Since the barriers between the isomers are of the order of $2 \mathrm{kcal} / \mathrm{mol}$, they can be easily overcome at room temperature over the time-scales of the action spectroscopy experiments [5] with trapping times of $40 \mathrm{~ms}$. The various conformers can therefore coexist at room temperature but are their excitations compatible with the spectral features observed in dissociation spectroscopy?

To answer this question, we compute the excitation energies of the RPSB conformers with two highly correlated approaches, namely, the CASPT2 method also employed for the ground state, and the n-electron valence-state perturbation theory (NEVPT2) [29]. The recently developed NEVPT2 method is more advanced than CASPT2 and generally more accurate. The key results are summarized in Table 5.1, where we report the vertical excitations computed on our M06-2X structures of the 6s-cis and 6s-trans conformers. Both methods predict that the excitations of the three conformers are comparable, only differing by about $30 \mathrm{~nm}$. Moreover, the vertical excitations are clustered on the blue edge of the dissociation spectrum in the range $520-560 \mathrm{~nm}$. Therefore, these highly correlated approaches strongly support that the excitations of the three conformers are comparable and do not span the experimental plateau extending between 530 and $610 \mathrm{~nm}$.

Table 5.1: Vertical excitation energies $(\mathrm{nm} / \mathrm{eV})$ of the retinal conformers, computed with CASPT2 and NEVPT2.

\begin{tabular}{llll}
\hline Geometry & Conformer / $\phi$ & CASPT2 & NEVPT2 \\
\hline M06-2X & trans / - 170.1 & $561 / 2.21$ & $551 / 2.25$ \\
& cis(-) / -38.3 & $537 / 2.31$ & $530 / 2.34$ \\
& cis(+) / 41.8 & $532 / 2.33$ & $523 / 2.37$ \\
CASSCF $^{a}$ & trans / - 185.7 & $544 / 2.28$ & $566 / 2.19$ \\
& cis(-) / -68.2 & $473 / 2.62$ & $477 / 2.60$ \\
\hline
\end{tabular}

${ }^{a}$ Geometries from Ref. 5.

In Table 5.1, we also report the excitations computed on the CASSCF geometries from Ref. 5 since the interpretation of the dissociation spectrum in terms of temperature effects was based on the use of these geometries. The excitations of the 6s-trans and 6s-cis conformers are different and the value for the cis orientation estimated between $473-477 \mathrm{~nm}$ falls outside the experimental plateau. We note that 
our CASPT2 excitations differs significantly from the values of 547 and $620 \mathrm{~nm}$ obtained in Ref. 5 for the cis and the trans conformers, respectively, with the use of a different zero-order Hamiltonian. The IPEA Hamiltonian [28] employed in this work yields on average more accurate excitations, and its superior performance is here corroborated by the good agreement found between our CASPT2 values and the NEVPT2 excitations. Consequently, we stress once again that our calibration of the method to generate the structures as well the excitations clearly indicates that CASSCF is not suitable to describe the structural features of RPSB.

Even though the conformers of retinal are characterized by similar excitations clustering at the blue edge of the spectrum, it is still possible that retinal may visit configurations with red-shifted excitations while fluctuating between the different orientations of the $\beta$-ionone. To investigate this possibility, we compute the excitation along the constrained path between the two ground-state minima as reported in Figure 5.4. We observe that, as retinal rotates between the trans and the cis configurations, the excited-state energy either roughly parallels the ground-state curve or raises even further, yielding higher values for the excitation. Therefore, temperature fluctuations can induce retinal to visit conformations characterized by different angles of the $\beta$-ionone ring but this motion will result in blue-shifted excitations at wavelengths shorter than $520 \mathrm{~nm}$. In summary, temperature fluctuations of the $\beta$-ionone ring cannot explain the broad feature in the spectrum.

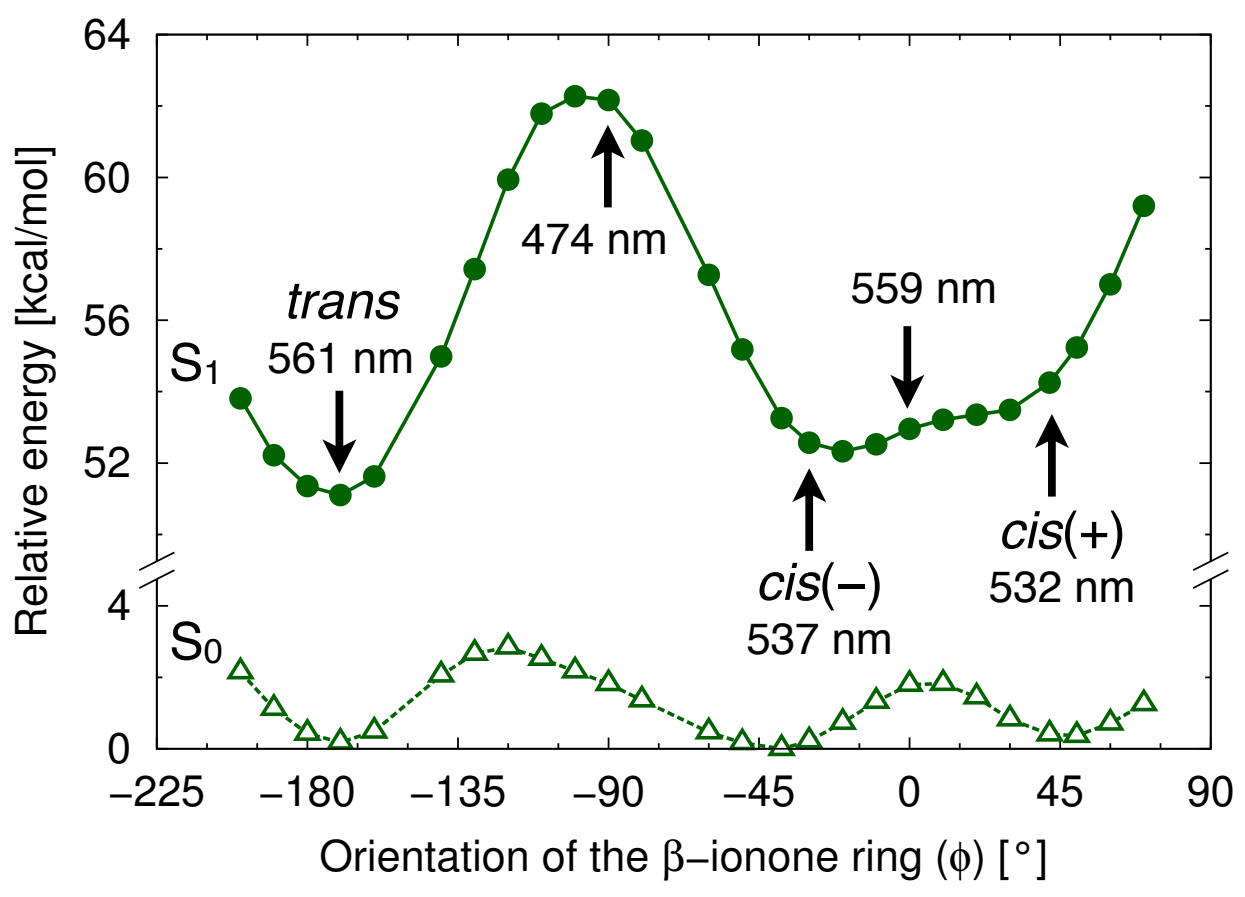

Figure 5.4: Ground- and excited-state energies computed with CASPT2 on top of M06-2X ground-state geometries. 
An obvious question is whether we have correctly identified the $\beta$-ionone angle as being the degree of freedom responsible for the anomalous broadening of the absorption spectrum. To investigate the possible influence of other active coordinates, we perform a room-temperature ab-initio molecular dynamics simulation of retinal in the cis(-) configuration for about 23 ps. This study is computationally feasible if we employ a generalized gradient approximation ( $0 \%$ exchange), and gives us a good indication of the most relevant degrees of freedom at finite temperatures. In agreement with previous studies [30], our dynamics reveals the particular activity of three coordinates: i) the global bend of the chromophore, which can be monitored via the $\mathrm{C}_{6}-\mathrm{N}_{16}$ distance, ii) the dihedral angle $\mathrm{C}_{10}-\mathrm{C}_{11}-\mathrm{C}_{12}-\mathrm{C}_{13}$, and iii) the length of the $\mathrm{C}_{11}-\mathrm{C}_{12}$ bond, which is inversely correlated with the bond length alternation in retinal (see Figure 5.5). We then further analyze these specific coordinates with more accurate approaches. In particular, we employ the M06-2X functional to compute a representative optimal path for constrained values of the coordinate in a range centered on the cis(-) minimum plus/minus twice the fluctuations as estimated along the molecular dynamics run (see SI [25]). Along these three paths, we then evaluate the CASPT2 excitations. As shown in Figure 5.6, the variations in these three coordinates do not significantly affect the excitations. A red shift of less than $10 \mathrm{~nm}$ is observed when the $C_{11}-C_{12}$ bond is stretched in correspondence to a bond length alternation shorter by about $0.02 \AA$. A similar red shift is obtained for an increased $C_{6}-N_{16}$ distance, while varying the $C_{10}-C_{11}-C_{12}-C_{13}$ dihedral angle does not affect the excitation. Consequently, not only the different orientations of the $\beta$-ionone ring but also the other degrees of freedom identified as most active at room temperature do not lead to the required red shift to explain the plateau in the photo-induced destruction absorption spectrum.

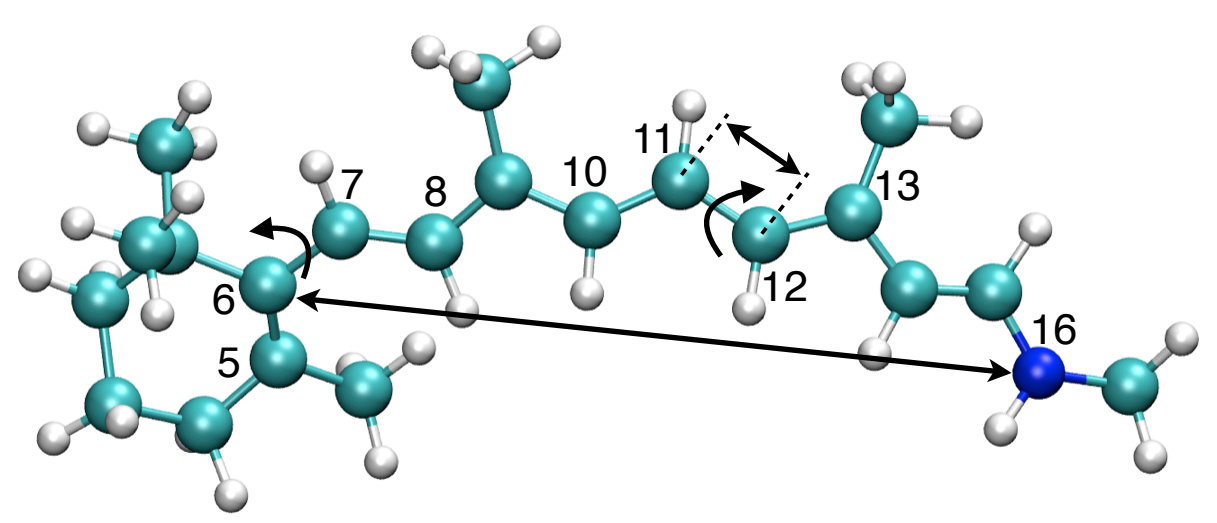

Figure 5.5: Active coordinates at room temperature: The global bend of the chromophore monitored via the $\mathrm{C}_{6}-\mathrm{N}_{16}$ distance, the dihedral angles $\mathrm{C}_{5}-\mathrm{C}_{6}-\mathrm{C}_{7}-\mathrm{C}_{8}$ and $\mathrm{C}_{10}-\mathrm{C}_{11}-\mathrm{C}_{12}-\mathrm{C}_{13}$, and the $\mathrm{C}_{11}-\mathrm{C}_{12}$ bond length. 


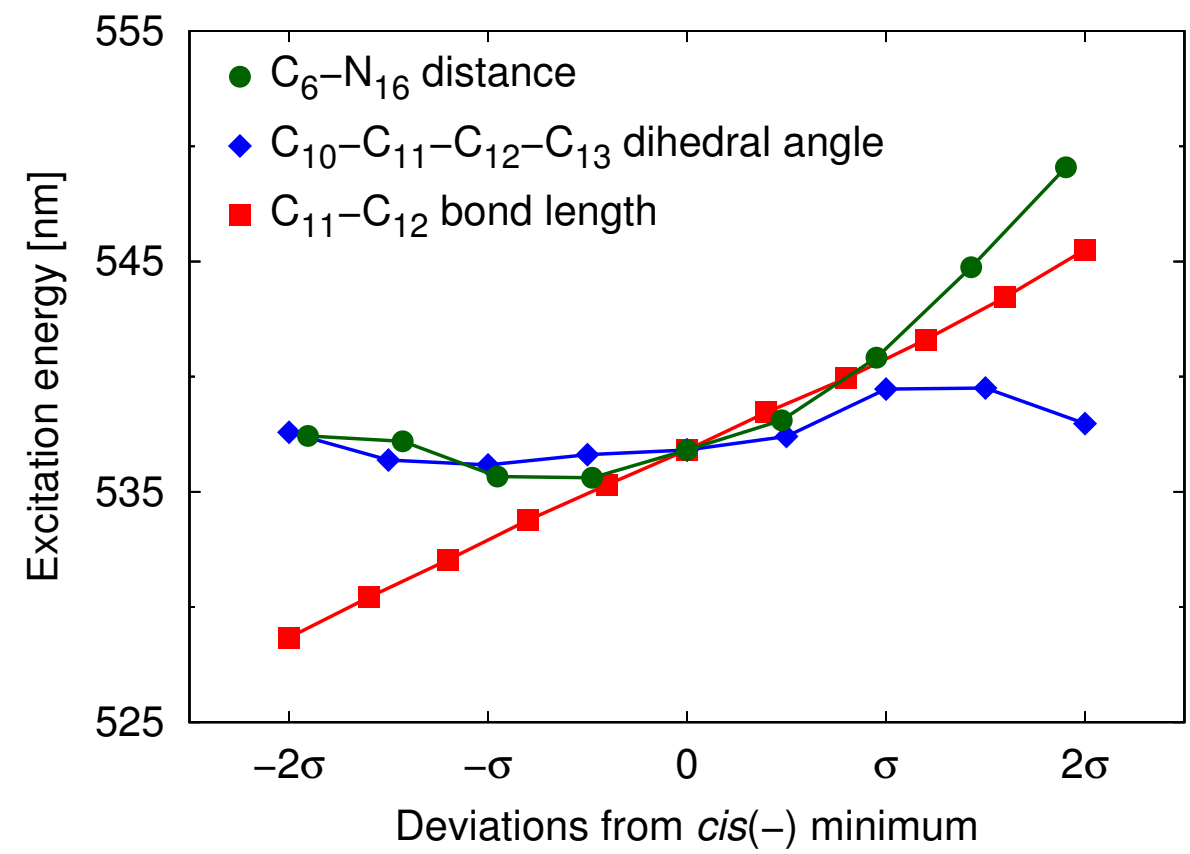

Figure 5.6: Excitation energies along the coordinates most active at room temperature, computed with CASPT2. The coordinates are centered at the cis(-) minimum and their room-temperature fluctuations $(\sigma)$ are $0.2 \AA$ for the $\mathrm{C}_{6}-\mathrm{N}_{16}$ distance, 0.025 $\AA$ for the $\mathrm{C}_{11}-\mathrm{C}_{12}$ bond length, and $10^{\circ}$ for the $\mathrm{C}_{10}-\mathrm{C}_{11}-\mathrm{C}_{12}-\mathrm{C}_{13}$ dihedral angle.

\subsection{Discussion and Conclusion}

In summary, we have employed here state-of-the-art first-principle approaches to investigate the absorption properties of RPSB in the gas phase and the impact of temperature effects on the spectrum. We first demonstrated the fundamental importance to carefully calibrate the method employed to generate the structure of this chromophore, and found that M06-2X represents an accurate tool for this purpose in contrast to the commonly used CASSCF method, which we proved is inadequate. We then investigated the different conformations of the $\beta$-ionone ring, which are found to be separated by low barriers and therefore easily accessible at room temperature. These conformers are however characterized by rather similar excitations and clustered at the blue edge of the photo-dissociation spectrum. Consequently, the different orientations of the ring cannot be responsible for the observed broad and flat plateau in the absorption spectrum. In addition, other degrees of freedom that we identify as active in room-temperature molecular dynamics simulations do not lead to the shift required to explain the anomalous spectral shape in terms of temperature effects.

While the disagreement between our theoretical results and photo-destruction experiments may appear distressing, we recall that experiments with dissociation 
spectroscopy appear to be plagued by potential complications such as the possible presence of multi-photon dissociation channels and the consequent non-trivial dependence of the shape of the spectrum on the excitation laser power $[15,16]$. In recent years, rather distinct spectra have been obtained for the same chromophore [13$15]$, retinal included $[4,5]$, so that the correlation between dissociation and optical spectra is becoming less clear with time. Our calculations for RPSB raise serious concerns on the interpretation of photo-induced dissociation spectroscopy experiments also for retinal. They provide compelling evidence that temperature effects cannot be responsible for the flat plateau between 530 and $610 \mathrm{~nm}$ in the spectrum, and that these model experiments cannot be considered representative of the optical spectrum of retinal. Our theoretical findings as well as the significant difference between available photo-dissociation spectra of retinal [4,5] call for further experimental investigations and careful characterization of these spectra in particular through intensity dependence studies.

\subsection{Bibliography}

[1] T. Ebrey and Y. Koutalos, Prog. Retinal Eye Res. 20, 49 (2001).

[2] B. Nickle and P. R. Robinson, Cell. Mol. Life Sci. 64, 2917 (2007).

[3] J. L. Spudich, C.-S. Yang, K.-H. Jung, and E. N. Spudich, Annu. Rev. Cell Dev. Biol. 16, 365 (2000).

[4] I. Nielsen, L. Lammich, and L. Andersen, Phys. Rev. Lett. 96, 018304 (2006).

[5] J. Rajput, D. Rahbek, L. Andersen, A. Hirshfeld, M. Sheves, P. Altoè, G. Orlandi, and M. Garavelli, Angew. Chem. Int. Ed. 49, 1790 (2010).

[6] S. Sekharan, O. Weingart, and V. Buss, Biophys. J. 91, L07 (2006).

[7] A. Cembran, R. González-Luque, L. Serrano-Andrés, M. Merchán, and M. Garavelli, Theor. Chem. Acc. 118, 173 (2007).

[8] K. Bravaya, A. Bochenkova, A. Granovsky, and A. Nemukhin, J. Am. Chem. Soc. 129, 13035 (2007).

[9] A. Altun, S. Yokoyama, and K. Morokuma, J. Phys. Chem. B 112, 16883 (2008).

[10] O. Valsson and C. Filippi, J. Chem. Theory Comput. 6, 1275 (2010).

[11] R. Send, V. R. I. Kaila, and D. Sundholm, J. Chem. Theory Comput. 7, 2473 (2011).

[12] K. Welke, J. S. Frähmcke, H. C. Watanabe, P. Hegemann, and M. Elstner, J. Phys. Chem. B 115, 15119 (2011). 
[13] S. B. Nielsen, A. Lapierre, J. U. Andersen, U. V. Pedersen, S. Tomita, and L. H. Andersen, Phys. Rev. Lett. 87, 228102 (2001).

[14] M. W. Forbes and R. A. Jockusch, J. Am. Chem. Soc. 131, 17038 (2009).

[15] K. Chingin, R. M. Balabin, V. Frankevich, K. Barylyuk, R. Nieckarz, P. Sagulenko, and R. Zenobi, Int. J. Mass Spectrom. 306, 241 (2011).

[16] M. W. Forbes, A. M. Nagy, and R. A. Jockusch, Int. J. Mass Spectrom. 308, 155 (2011).

[17] J. Olsen, Int. J. Quantum Chem. 111, 3267 (2011).

[18] H. C. Watanabe, Y. Mori, T. Tada, S. Yokoyama, and T. Yamato, BIOPHYSICS 6, 67 (2010).

[19] A. D. Becke, Phys. Rev. A 38, 3098 (1988).

[20] C. Lee, W. Yang, and R. G. Parr, Phys. Rev. B 37, 785 (1988).

[21] A. D. Becke, J. Chem. Phys. 98, 5648 (1993).

[22] P. J. Stephens, F. J. Devlin, C. F. Chabalowski, and M. J. Frisch, J. Phys. Chem. 98, 11623 (1994).

[23] Y. Zhao and D. Truhlar, Theor. Chem. Acc. 120, 215 (2008).

[24] M. Wanko, M. Hoffmann, P. Strodel, A. Koslowski, W. Thiel, F. Neese, T. Frauenheim, and M. Elstner, J. Phys. Chem. B 109, 3606 (2005).

[25] Supporting information (SI), can be found at http://dx.doi.org/10.1021/jz300183g.

[26] See Figure S2 in the SI for a discussion of the discrepancy with Ref. 5 regarding the CASSCF geometries.

[27] P. Pulay, Int. J. Quantum Chem. 111, 3273 (2011).

[28] G. Ghigo, B. O. Roos, and P.-Å. Malmqvist, Chem. Phys. Lett. 396, 142 (2004).

[29] C. Angeli, M. Pastore, and R. Cimiraglia, Theor. Chem. Acc. 117, 743 (2007).

[30] U. F. Röhrig, L. Guidoni, and U. Rothlisberger, ChemPhysChem 6, 1836 (2005). 



\section{Chapter 6}

\section{Native Description of Visual Absorption}

\subsection{Introduction}

Rhodopsin [1,2] is the visual pigment responsible for dim light vision and is the most extensively studied member of the family of visual opsin proteins, a large class of G-protein-coupled receptors that respond to light and initiate the visual transduction process in vertebrate $[3,4]$. Most visual opsins share the same covalently linked chromophore, the retinal protonated Schiff base (RPSB) molecule, while variations in the chromophore-protein interaction tune the absorption maximum over a very

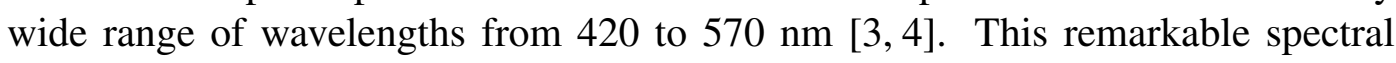
tuning has prompted a large number of experimental [1-31] and theoretical [31-58] studies that have attempted to understand the molecular mechanism underlying the functioning of the visual opsins.

Due to the complexity of system, obtaining an accurate theoretical description of the absorption in visual opsins does not appear to be a trivial task since we might expect a strong dependence of the excitation energies on the various ingredients entering in the calculations. Nevertheless, for Rhodopsin, many theoretical studies have reported a remarkable agreement with the experimental absorption maximum even though these studies often differ considerably in the theoretical approaches employed and normally resort to various approximations in the treatment of the chromophore-protein interaction. If we analyse these theoretical investigations more closely, we discover that they often lead to very different conclusions as regards the role of the protein environment in tuning the excitation energy of the chromophore. A particularly interesting case is given by the studies of Ref. 34 and 57, which obtain excitation energies of $2.47 \mathrm{eV}$ and $2.42 \mathrm{eV}$, respectively, in rather good agreement with the experimental absorption maximum at $498 \mathrm{~nm}(2.49 \mathrm{eV})$ [5]. At first sight, these calculations appear to be quite similar since they start from the same crystallographic structure and employ the same multi-reference perturbative approach (CASPT2) to obtain the excitation. However, they reach very different conclusions concerning the electrostatic effect of the protein environment on the excitation en- 
ergy of the chromophore. In Ref. 34, the main effect of the protein is due to the counter-ion, which induces a significant blue shift of about $0.5 \mathrm{eV}$, while the rest of the protein environment has a negligible effect. On the other hand, Ref. 57 obtains a much larger blue shift of $1.2 \mathrm{eV}$ due to the counter-ion, which is then quenched by the rest of the protein environment (see Figure 1.5). These two studies employ different methods to optimize the structural model of the system, which result in different chromophore geometries. Differences in other aspects of the calculations (e.g. protonation state of some residues) might further affect the response of the excitation to the protein environment. Therefore, since these (and others) contradicting studies cannot all be right, the apparent agreement with experiments is in most cases due to a favorable cancelation of errors.

Despite the numerous theoretical investigations, it remains therefore unclear whether we are in a position to accurately describe the absorption of opsins from first principles and whether we have identified all necessary ingredients to achieve this goal. It is clear that one should use an accurate approach to compute the excitations but, even if we restrict ourselves to highly-correlated methods, this choice is far from obvious as the various techniques yield very different results even for the retinal chromophore in the gas phase [59]. Furthermore, for the description of the chromophore-protein interaction, it has been pointed out that, to obtain accurate absorptions, the mutual polarization of the chromophore and the protein environment should be be considered [40-42]. Nevertheless, the generally accepted procedure is to employ a standard quantum mechanics in molecular mechanics $(\mathrm{QM} / \mathrm{MM})$ approach, where only the chromophore is described at the quantum level while the rest of the protein environment is modeled by employing fixed, partial point charges, normally taken from a particular classical force field. In this multiscale scheme, the point charges polarize the quantum chromophore in its ground and excited states, whereas the environment does not respond to the presence of the chromophore. Finally, another important aspect of modeling an opsin is to account for the dynamical nature of the protein since thermal fluctuations will affect the structure, the chromophore-protein interaction, and the resulting excitation energy. This point has been largely ignored in most previous theoretical studies, which just consider a single, static model of the protein. Furthermore, the protein is often taken to be the available crystallographic structure, where only the chromophore or a small region around it is relaxed, while the rest of the protein is kept fixed at the crystallographic positions. The investigations which attempt to include thermal fluctuations do so in a limited way, either by only performing classical molecular dynamics (MD) followed by QM/MM geometrical optimization [47-49], or by performing QM/MM MD where a part of the protein is kept fixed [50-52].

Here, we will explore these issues and establish which theoretical ingredients are needed for an accurate description of the absorption of Rhodopsin. To achieve this goal, we will employ a realistic model of Rhodopsin embedded in its native membrane environment (see Figure 6.1), which was carefully equilibrated with classical MD [60]. For this model system, we will perform extensive and fully unconstrained QM/MM MD simulations to obtain an accurate structural description of the chro- 
mophore and of the thermal fluctuations of the chromophore-protein system at room temperature. An absorption spectrum obtained at room temperature with a cheaper but more approximate approach will allow us to select a set of geometrical configurations close to the middle of the band, for which we will compute the excitations at a higher level of theory. In this step, we will not a priori assume the correctness of a particular theoretical approach but carefully calibrate the computation of the excitation energies with a wide range of highly-correlated methods. Furthermore, with the use of enlarged quantum regions, we will explore whether the conventional QM/MM scheme used to compute the excitation energies properly accounts for the effect of the protein environment. Finally, we will compare our results with the simpler theoretical procedure so often adopted in previous Rhodopsin studies [53-58], and explain the reasons for the remarkable, fortuitous agreement with experiments achieved in these studies.

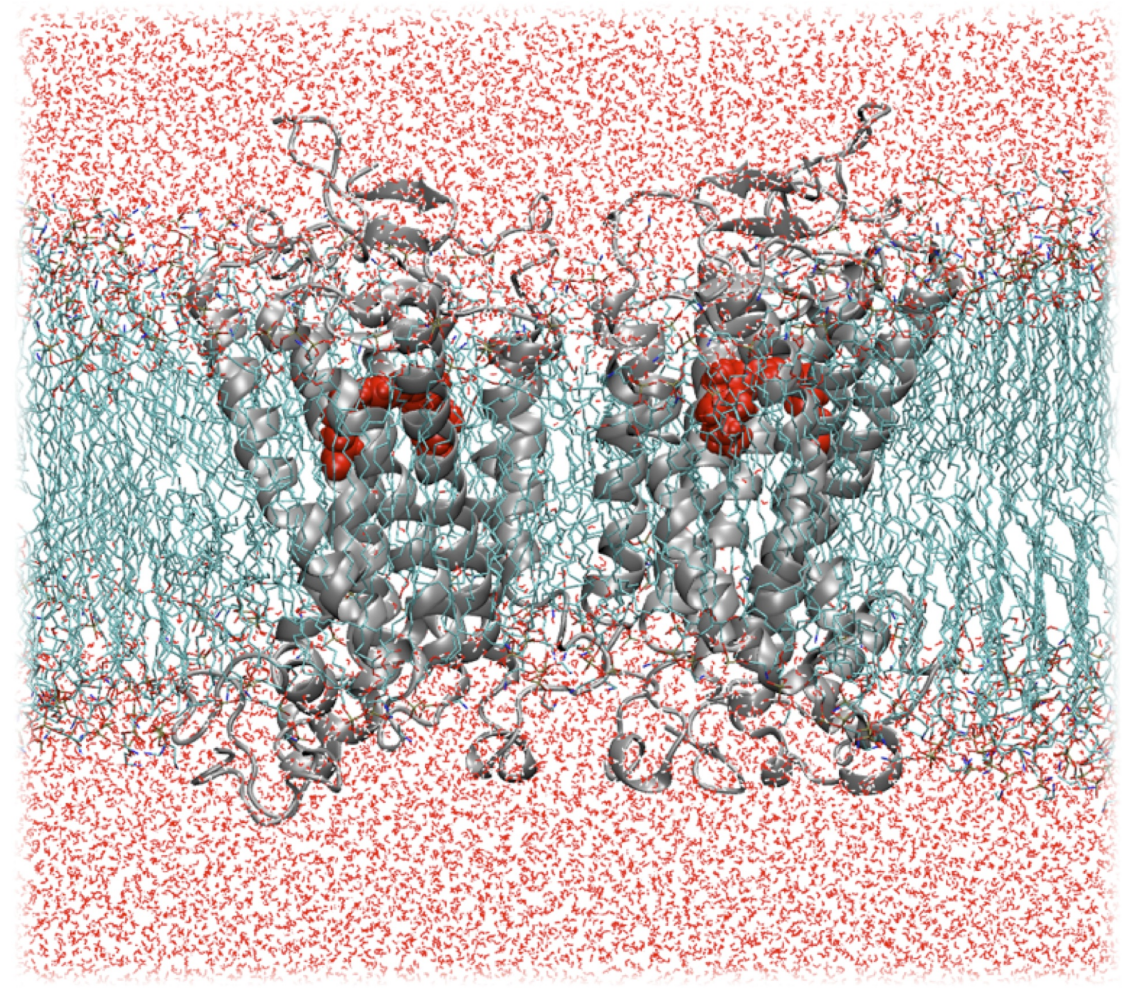

Figure 6.1: The Rhodopsin dimer system used in the current work. The dimer is embedded in an explicit membrane and the extracellular side is facing up. For clarity, the hydrogens have been removed from the lipids. 


\subsection{Methods}

\subsubsection{Model System}

Our model system of the bovine Rhodopsin dark state is taken from a previous study [60], and consists of two Rhodopsin monomers embedded in an explicit membrane. As described in Ref. 60, the Rhodopsin dimer was equilibrated via classical MD for 300 ns. We will give below the most relevant details of our model system and refer the reader to Ref. 60 for further details.

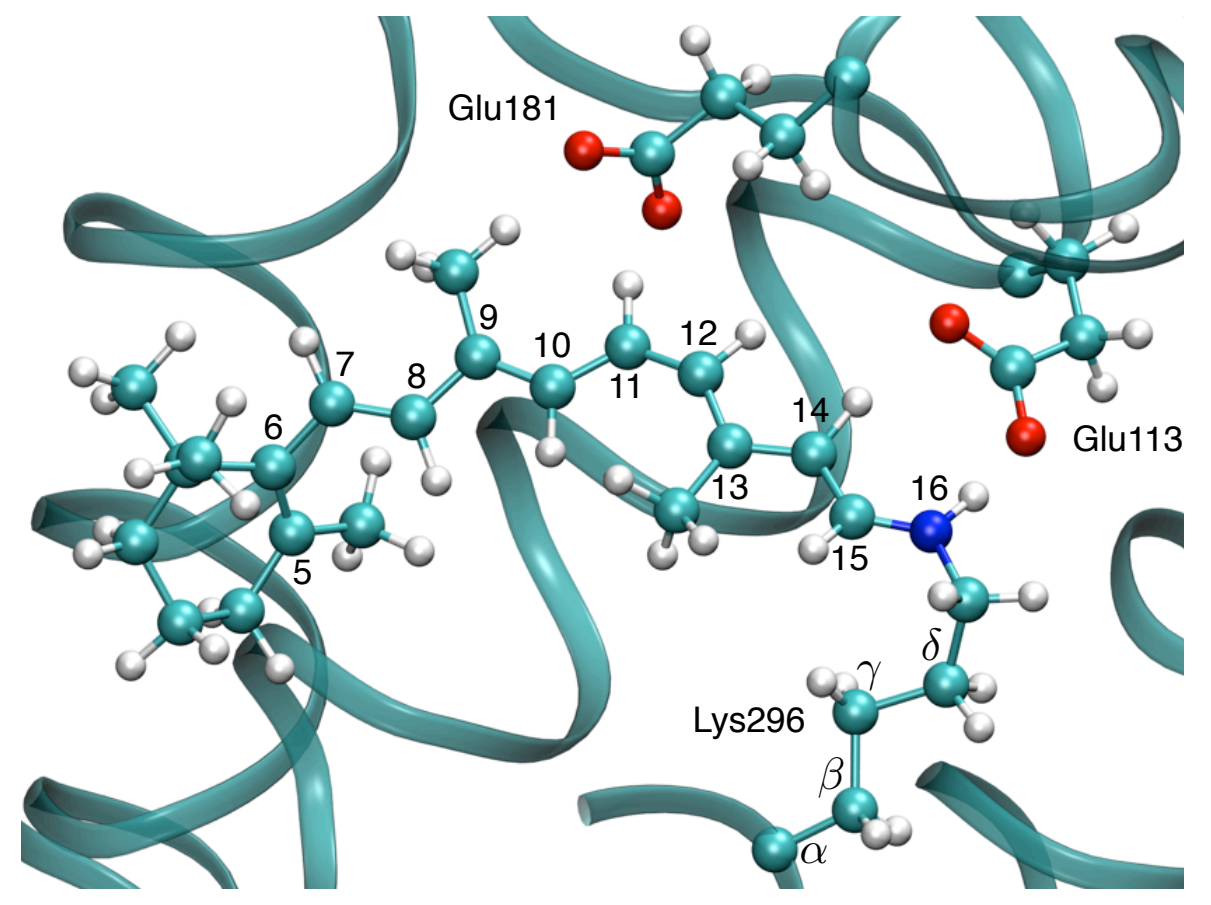

Figure 6.2: The retinal protonated Schiff base (RPSB) with the Glu113 counter-ion and the Glu181 residue. We also show the numbering used for the conjugated chain of the RPSB $\left(\mathrm{C}_{5}, \mathrm{C}_{6}, \ldots, \mathrm{C}_{15}, \mathrm{~N}_{16}\right)$ and indicate the side chain carbons of the Lys296 residue.

For the protein, all potentially charged amino acids, including the $\mathrm{C}$ - and $\mathrm{N}$ termini, are considered to be in their default protonation states except Asp83 and Glu122 that are assumed to be neutral in line with FTIR experiments [19]. In particular, the Glu181 residue is deprotonated (charged). Histidine residues are considered to be protonated either at the $\mathrm{N}_{\delta}$ position (His100, His211) or the $\mathrm{N}_{\epsilon}$ position (His65, His 152, His195, His278). The retinal chromophore is covalently linked to the Lys296 residue via a protonated Schiff base linkage, which we collectively call the retinal protonated Schiff base (RPSB), as shown in Figure 6.2. The Cys110 and Cys187 residues are linked via a disulfide bond and two palmitic acid residues, which act as membrane anchoring points, are bound to Cys322 and Cys323. As shown in Figure 6.1, the Rhodopsin dimer is embedded in an explicit membrane 
modeled by 1-stearoyl-2-oleoyl-sn-glycero-3-phosphoethanolamine (SOPE) lipids while explicit water molecules cover the cytoplasmic and extracellular sides. To obtain an overall neutral system, four sodium ions are added. The system is placed in a periodic cubic box with approximate dimensions of $130 \times 80 \times 100 \AA^{3}$ with around 20000 waters and 300 lipids, which result in a complete model system of around 100000 atoms. The waters are described using the TIP3P force field [61] and the AMBER/parm99 force field [62] is employed for all standard protein residues. The force fields for the RPSB and the palmitic acid residues are described in Ref. 63, while the AMBER/parm96 force field is used for the SOPE lipids. As described in Ref. 60, the Rhodopsin dimer system model was equilibrated via classical MD for $300 \mathrm{~ns}$ in the NPT ensemble at a temperature of $310 \mathrm{~K}$ and a pressure of $1.05 \mathrm{bar}$.

\subsubsection{QM/MM MD Simulations}

In order to construct an unbiased starting structure for the QM/MM MD simulations, we perform a clustering analysis [64] on the last $100 \mathrm{~ns}$ of the classical MD equilibration run. Each trajectory frame is ranked based on the number of neighboring frames that are within $1 \AA$ RMSD cut-off. The top-ranked frame and all its neighboring frames then form a cluster. The frames corresponding to this cluster are then removed and the ranking process repeated until all frames are assigned to a given cluster. This results in a set of non-overlapping clusters of frames. We then use the central structure from the most populated cluster as a starting structure for our QM/MM MD simulations.

We perform the QM/MM MD simulations using the QM/MM scheme implemented in the CP2K package [65]. This QM/MM scheme employs the Quickstep QM code [66], the Fist MM driver, and a real-space multigrid technique for the electrostatic coupling between the QM and MM regions [67,68]. The QM region is treated within density functional theory (DFT), while the MM region is modeled using the AMBER force field described above. The DFT calculations are performed in the generalized gradient approximation by employing the PBE [69] exchangecorrelation functional. In the DFT calculations, a mixed Gaussian and plane waves (GPW) approach is used, where the wave function is described with a triple-zeta valence basis set augmented with two sets of polarization functions (TZV2P) [70], while the electron density is converged employing an auxiliary plane-wave basis set with a density cutoff of 320 Ry. The Goedecker-Teter-Hutter (GTH) pseudopotentials $[71,72]$ are employed to describe the core electrons.

Only the chromophore located in one of the two monomers is included in the QM region while the other, together with the rest of the protein environment, is treated at the MM level. We perform three different QM/MM MD simulations that differ in the residues included in the QM region: Rho-1, the retinal protonated Schiff base (RPSB); Rho-2, RPSB and the Glu113 counter-ion; Rho-3, RPSB, Glu113, and Glu181. For the Glu113 and Glu181 residues, the QM/MM boundary is along $\mathrm{C}_{\alpha^{-}}$ $\mathrm{C}_{\beta}$ bond, while for the RPSB the boundary is along the $\mathrm{C}_{\alpha}-\mathrm{C}_{\beta}$ bond of the Lys296 residue. We saturate the valency of the $\mathrm{QM}$ regions by adding hydrogen capping 
atoms to the carbon QM atoms at the QM/MM boundary. All simulations are performed in the Born-Oppenheimer (BOMD) approximation within the ab initio MD framework and with a time step of $0.5 \mathrm{fs}$. In each simulation, the QM region is initially optimized while the MM part is kept frozen. After thermalization and further equilibration, data collection is started and each simulation is run for 15 ps in the canonical (NVT) ensemble. A stochastic velocity rescaling thermostat [73] is employed to maintain the temperature at $300 \mathrm{~K}$. To compute the geometrical averages and the Zindo excitations, we use the last $7.5 \mathrm{ps}, 7.3 \mathrm{ps}$, and $11.3 \mathrm{ps}$ of the Rho-1, Rho-2, and Rho-3 runs, respectively, and collect frames every 5 fs (10 time steps) so that the total number of frames is 1491,1450 , and 2248 , respectively.

\subsubsection{Excited-State Calculations}

For the Zindo calculations, we employ the Gaussian 09 code [74] with the default settings. The TDDFT calculations with CAM-B3LYP [75] and LC- $\omega$ PBE $[76,77]$ functionals are performed using the Gaussian 09 code and the $6-31+\mathrm{G}^{*}$ basis set. For the CC2 calculations, we use the TURBOMOLE code [78] where we employ the resolution-of-the-identity (RI) approximation [79], the ANO-L-VDZP [80] basis set, and the aug-cc-pVTZ auxiliary basis set [81,82].

The CASPT2 calculation are performed with the MOLCAS [83] code. Unless otherwise stated, we use the default IPEA zero-order Hamiltonian [84] and report the single-state (SS) CASPT2 values. We use a constant imaginary level shift [85] of 0.1 a.u. and employ the ANO-L-VDZP basis set. For the CAS active space, we include all $12 \pi$ electrons in the reference, and either 12 or 13 active $\pi$ orbitals, resulting in a CAS $(12,12)$ or $\operatorname{CAS}(12,13)$ expansion. We use a state-average (SA) CASSCF wave function with equal weights over either the two $\left(\mathrm{S}_{0}\right.$ and $\left.\mathrm{S}_{1}\right)$ or three $\left(\mathrm{S}_{0}, \mathrm{~S}_{1}\right.$, and $\left.\mathrm{S}_{2}\right)$ lowest-energy states.

For the NEVPT2 calculations, we use the strongly contracted (SC) variant [86$88]$ as implemented in the ORCA $2.8[89,90]$ code, and use the same basis set and SA-CASSCF wave function as in the CASPT2 calculations. The DMC calculations are performed with the CHAMP [91] code. In all DMC calculations, we use a CAS(12,12) expansion, SA-CASSCF wave functions optimized over two states with equal weights, and pseudopotentials with the corresponding double zeta basis set [92] and with added s and $p$ diffuse functions on the heavy atoms.

In the QM/MM excited-state calculations, we employ two different QM regions, one including only the chromophore (RPSB) and the another including the chromophore and the Glu113 counter-ion (RPSB+Glu113). For the RPSB, the QM/MM boundary is along $\mathrm{C}_{\gamma}-\mathrm{C}_{\delta}$ bond of the Lys296 residues and for Glu113 it is along the $\mathrm{C}_{\alpha}-\mathrm{C}_{\beta}$ bond. The valency of the $\mathrm{QM}$ region is saturated by adding hydrogen capping atoms at the QM/MM boundary. The MM environment is modelled by employing partial point charges for MM atoms with the parameters taken from the AMBER force field of the system described above. To avoid over-polarization of the QM region, we turn off the point charges for a small group of $\mathrm{MM}$ atoms at the QM/MM boundary. 


\subsection{Results}

\subsubsection{QM/MM MD Simulations}

As well know, the timescales accessible in QM/MM MD simulations are orders of magnitude smaller than what is possible in classical MD simulations. This makes the selection of a starting point in a QM/MM MD simulation a critical issue as the initial structure must be representative of an average configuration of the system. In this work, we make this selection in a unbiased way by using clustering analysis on a $100 \mathrm{~ns}$ classical MD simulation to identify an initial structure. Starting from this selected structure, we perform three different QM/MM MD simulations at room temperature that differ in the QM region used: Rho-1 has the minimal QM region

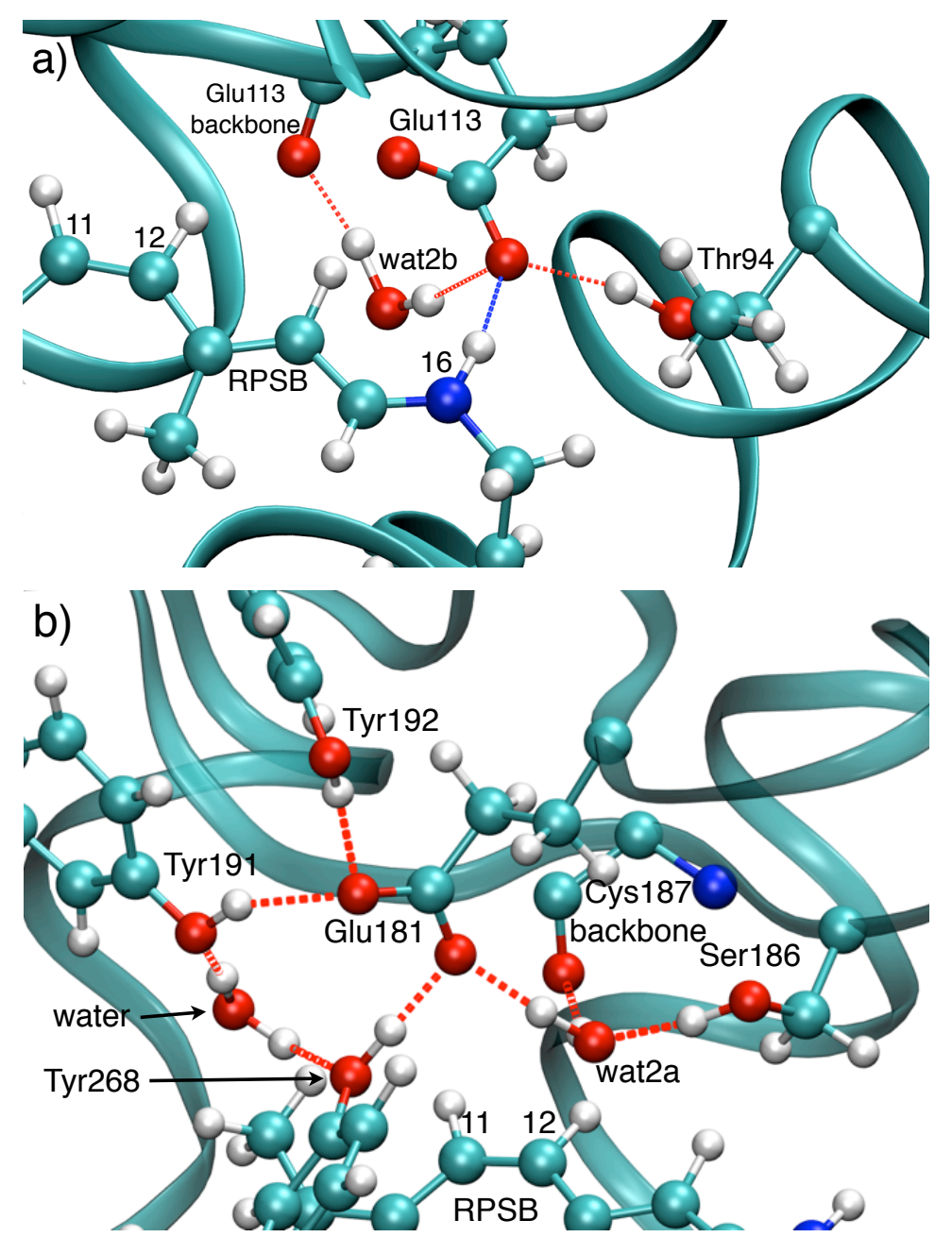

Figure 6.3: The hydrogen bonding network $(\mathrm{HBN})$ around the chromophore in the protein pocket: a) The HBN around the Glu113 counter-ion; b) the HBN around the Glu181 residue. 
of the RPSB, Rho-2 adds the Glu113 counter-ion to the QM region, and Rho-3 additionally includes the Glu181 residue in the QM region. Each simulations is run for a total time of 15 ps while we use the last 7 to 11 ps for the analysis and calculations below.

All three QM/MM simulations show the same hydrogen bonding network (HBN) around the chromophore in the protein pocket, which is well preserved from the initial structure and stable throughout each simulation. The HBN can be divided into two parts, one around the Glu113 counter-ion and the other around the Glu181 residue. As shown in Figure 6.3a, the Glu113 counter-ion is hydrogen bonded to a crystallographic water, wat $2 \mathrm{~b}[16,17]$, and to Thr94, which is a residue known to be important for the stability of the protein pocket [21,22]. In the HBN around Glu181 shown in Figure 6.3b, one oxygen of Glu181 is hydrogen bonded to Tyr191 and Tyr192, while the other oxygen is hydrogen bonded to Tyr268 and a crystallographic water molecule, wat2a [15-17]. This water molecule also forms a hydrogen bond to Ser186 and the backbone of Cys187. Finally, another water molecule bridges the Tyr191 and Tyr268 residues.

In the Rhodopsin literature, there has been a long debate on the protonation state of Glu181, which we consider in our model system to be deprotonationed (charged). We will not delve into this issue and refer the reader for a more detailed discussion to Refs. 31 and 51, and references therein. However, we do note that throughout the 100 ns classical MM simulation, the HBN around the Glu181 residue is very stable, indicating that the protonation states used in our model, especially the one chosen for Glu181, are accurate. This is also consistent with a previous classical MD study [63] on a Rhodopsin monomer where a deprotonated Glu181 resulted in stable HBN while a protonated Glu181 resulted in a disrupted HBN.

In Table 6.1, we show the relevant geometrical parameters for the three QM/MM runs. For comparison, the geometrical parameters of the retinal chromophore optimized in the gas phase are presented in Table 6.3. As observed in the crystallographic structures and expected from the location in the protein pocket, the geometry of the chromophore is considerably distorted by the interactions with the protein environment with respect to the gas-phase geometry. Steric interactions in the protein pocket significantly distort the conjugated chain of the chromophore from planarity, as can be seen from the $\mathrm{C}_{11}-\mathrm{C}_{12}$ dihedral angle $\left(\phi_{\mathrm{C} 10-\mathrm{C} 11-\mathrm{C} 12-\mathrm{C} 13}\right)$. This is also observed in the effective length of the conjugated chain since the $\mathrm{C}_{6}-\mathrm{N}_{16}$ distance $\left(d_{\mathrm{C} 6-\mathrm{N} 16}\right)$ is about $0.3-0.5 \AA$ shorter than in the gas-phase geometry, indicating a more bent chromophore. Finally, the electrostatic interaction between the positively charged chromophore and the negatively charged Glu113 counter-ion induces an increased bond length alternation (BLA) along the conjugated chain of the chromophore.

We observe that the three runs differ considerably in the counter-ion distance $\left(d_{\text {counter-ion }}\right)$, which we define as the distance between the nitrogen on the RPSB and the closer carboxylate oxygen on the Glu113 counter-ion. For the Rho-1 run, the counter-ion distance is about $0.4 \AA$ longer than for the Rho- 2 and Rho-3 runs. This also influences the BLA, which has a slightly smaller average value in the Rho-1 run. 
Table 6.1: Relevant geometrical parameters (see Figure 6.2) and Zindo results for the QM/MM MD runs. Trajectory averages are shown together with their root mean square fluctuations. Distances are in $\AA$ and angles are in degrees. The BLA is defined as the difference between the averages of the single and double carbon-carbon bonds lengths, and computed including the bonds between $\mathrm{C}_{5}$ and $\mathrm{C}_{15}$. For the Gaussian fit to the Zindo absorption spectrum, we show the mean $(\mu)$, the standard deviation $(\sigma)$, and full width at half maximum (FWHM).

\begin{tabular}{|c|c|c|c|}
\hline & Rho-1 & Rho-2 & Rho-3 \\
\hline QM region & RPSB & $\begin{array}{c}\text { RPSB } \\
+ \text { Glu113 }\end{array}$ & $\begin{aligned} & \text { RPSB } \\
+ \text { Glu113 } & + \text { Glu181 }\end{aligned}$ \\
\hline$d_{\text {counter-ion }}$ & $3.23 \pm 0.10$ & $2.77 \pm 0.11$ & $2.77 \pm 0.11$ \\
\hline$d_{\mathrm{C} 6-\mathrm{N} 16}$ & $11.20 \pm 0.17$ & $11.19 \pm 0.13$ & $11.24 \pm 0.18$ \\
\hline BLA & $0.051 \pm 0.020$ & $0.059 \pm 0.020$ & $0.057 \pm 0.021$ \\
\hline$\phi_{\mathrm{C} 5-\mathrm{C} 6-\mathrm{C} 7-\mathrm{C} 8}$ & $-44.2 \pm 10.1$ & $-50.4 \pm 10.8$ & $-45.7 \pm 9.8$ \\
\hline$\phi_{\mathrm{C} 10-\mathrm{C} 11-\mathrm{C} 12-\mathrm{C} 13}$ & $-17.6 \pm 8.8$ & $-14.4 \pm 9.4$ & $-11.9 \pm 9.3$ \\
\hline \multicolumn{4}{|l|}{ Zindo $(\mathrm{S} 0 \rightarrow \mathrm{S} 1)$} \\
\hline a) Average & $2.34 \pm 0.10$ & $2.41 \pm 0.12$ & $2.42 \pm 0.13$ \\
\hline \multicolumn{4}{|c|}{ b) Gaussian fit to absorption spectrum } \\
\hline$\mu$ & 2.33 & 2.40 & 2.41 \\
\hline$\sigma$ & 0.11 & 0.12 & 0.13 \\
\hline FWHM & 0.25 & 0.28 & 0.30 \\
\hline
\end{tabular}

All the QM/MM runs show the same HBN and have the same starting point so it is clear that the difference lies in how the interaction between the chromophore and the counter-ion is described. In the Rho-1 run, we have a quantum-classical interaction while, in Rho-2 and Rho-3 runs, we have a quantum-quantum interaction. It is hard to fully judge which description leads to a more accurate counter-ion distance as comparison with other calculations and experiments yields a mixed message. Our classical MD simulation has an average counter-ion distance of $2.73 \pm 0.11 \AA$, and most other theoretical studies $[17,33,51,57]$ converge to a value of about $2.7 \AA$. On the other hand, all Rhodopsin crystal structures [14-17] have a counter-ion distance in the range of $3.1 \AA$ to $3.9 \AA$. Fortunately, we will see below that the increased counter-ion distance does not seem to significantly influence the excitation energies. Finally, we note that the Rho-2 and Rho-3 runs exhibit very comparable geometrical parameters and, as shown below, Zindo excitation energies. This indicates that is not needed to treat the charged Glu181 residue as quantum in the QM/MM MD simulations. 


\subsubsection{Zindo Absorption Spectra}

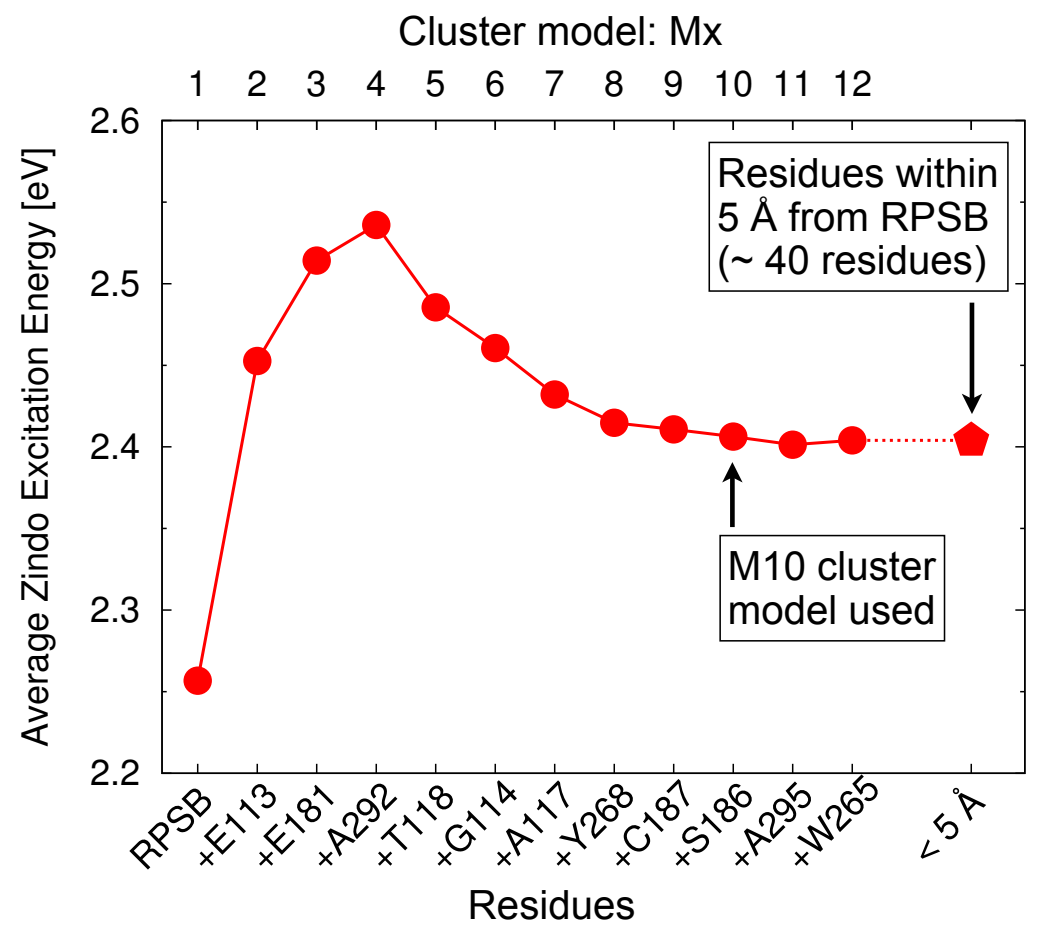

Figure 6.4: Convergence of the Zindo average excitation energy with the size of the cluster model. The smallest cluster includes only the chromophore (RPSB) and additional residues are then added one by one. We also consider a cluster which includes all residues within $5 \AA$ from the RPSB (about 40 residues and 720 atoms). The calculations are performed for a set 100 equispaced frames from the Rho- 2 run.

For each of the three QM/MM MD simulations, we extract 1500-2000 uncorrelated frames from the last $10 \mathrm{ps}$ of the simulations, which we use to obtain the absorption spectra at room temperature. This large number of frames puts some constraints on the excited-state approach we can use, which must give reasonable results and be economical. Here, we use the semi-empirical Zindo method, which surely fulfills the criterium of being affordable. Even though Zindo has shown a reasonable performance in benchmark studies, we need to keep in mind that Zindo often exhibits red-shifted excitation energies as compared to more accurate approaches [93]. Here, we use however the Zindo results mainly as a guide in identifying structures which are in the relevant region of phase space, namely, which give excitation energies close to the maximum of the theoretical absorption band.

The Zindo calculations are performed on cluster models that include the RPSB, the Glu113 counter-ion, and other nearby residues we find to be important for the absorption. To establish which cluster size gives converged excitations, we average the excitation energies computed on clusters of increasing size extracted from a sub- 
set of 100 equispaced frames from the Rho-2 run, and show the results in Figure 6.4. For the RPSB chromophore (M1 model), we obtain an average value of $2.26 \mathrm{eV}$ and adding the Glu113 counter-ion (M2 model) blue-shifts the average excitation energy to $2.45 \mathrm{eV}$. The inclusion of the Glu181 and Ala292 residues (M4 model) gives the highest excitation while, starting from the M5 model, each additional residue leads to a red shift. The average excitation energy levels off for models M10 to M12 to a converged value of $2.40 \mathrm{eV}$. We also consider an extended model given by all residues with at least one atom within $5 \AA$ of the RPSB (about 40 residues and 720 atoms), which yields an average excitation of $2.40 \mathrm{eV}$, that is, equal to the values obtained for the M10 to M12 clusters. It is therefore sufficient to use the M10 cluster model for the Zindo calculations of the excitation energies. The M10 model includes about 250 atoms and is shown in Figure 6.5.

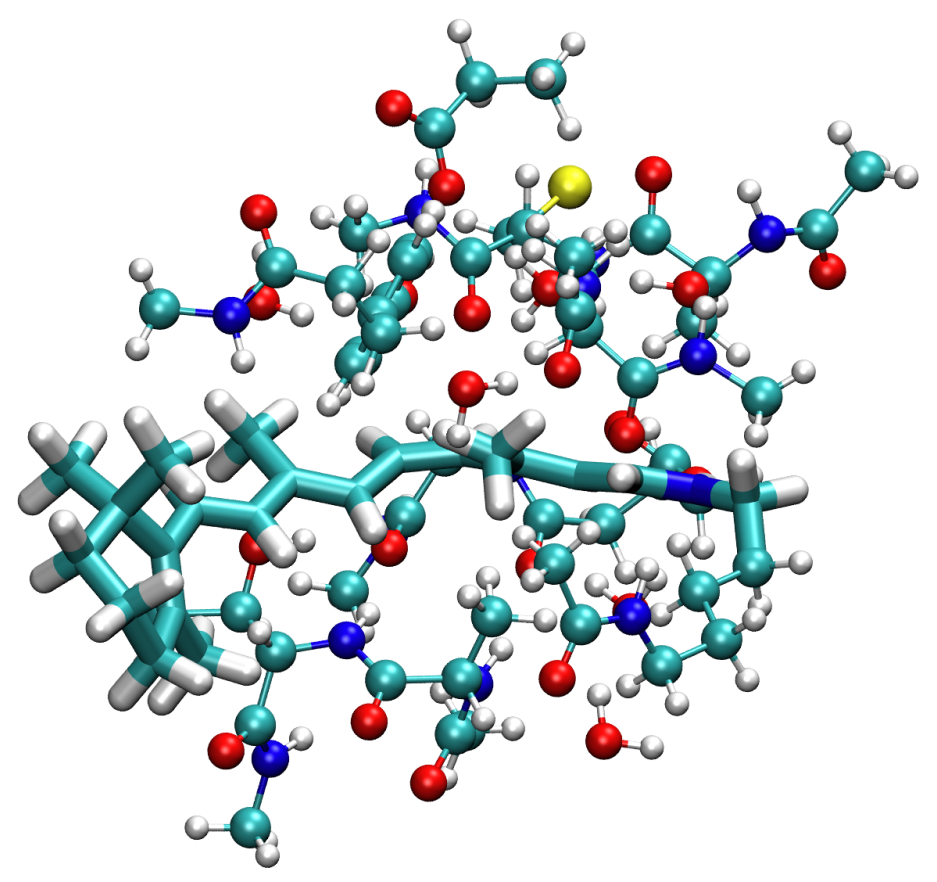

Figure 6.5: The M10 cluster model used in the Zindo calculations, which includes about 250 atoms.

With the M10 cluster, we now calculate the absorption spectrum by weighting the Zindo excitation energies with their oscillator strength for the full set of frames, and show the spectrum for the Rho-2 run in Figure 6.6. As shown in Table 6.1, we find that the average Zindo excitation energies for the three runs are very similar to the absorption maxima obtained in the Gaussian fits. Furthermore, the results for the Rho-2 and Rho-3 runs are almost equal, which is consistent with the observation above that the inclusion of Glu181 in the QM region of the QM/MM MD simulation does not influence the structure of the protein pocket. The spectrum for the Rho-1 run is instead slightly red shifted by about $0.07 \mathrm{eV}$, and this shift can be explained by the larger counter-ion distance and the slightly smaller BLA. 


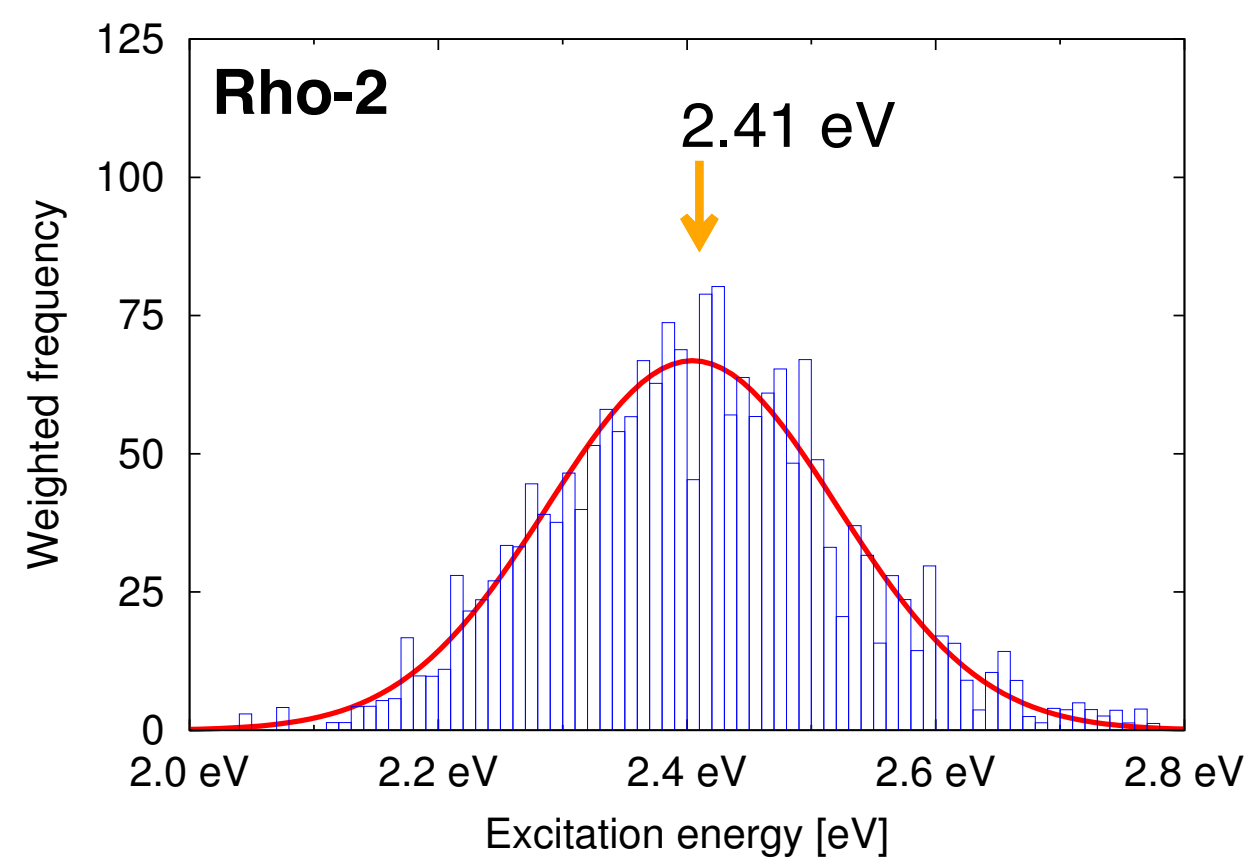

Figure 6.6: Zindo absorption spectrum for the Rho-2 trajectory obtained with a histogram where the excitations are weighted with oscillator strength (blue boxes). We show also a Gaussian fit of the histogram (red line). For the histogram, we use bins of $0.01 \mathrm{eV}$.

Finally, we note that, for the Rho-2 and Rho-3 simulations, the absorption maximum of about $2.4 \mathrm{eV}$ is very close to the experimental value of $2.49 \mathrm{eV}(498 \mathrm{~nm})$. Furthermore, for Rhodopsin, the inhomogeneous broadening at room temperature, (i.e. the broadening of the spectrum due to thermal fluctuations in the structure) has been estimated to be $0.16 \mathrm{eV} \mathrm{[26],} \mathrm{which} \mathrm{is} \mathrm{in} \mathrm{good} \mathrm{agreement} \mathrm{with} \mathrm{the} \mathrm{full} \mathrm{width} \mathrm{at}$ half maximum of $0.25-0.30 \mathrm{eV}$ for the Zindo spectra.

\subsubsection{TDDFT Excitations}

We employ time-dependent density functional theory (TDDFT) with the CAMB3LYP functional to refine the excitation energies of frames extracted from the region of the Zindo absorption maximum. We select eleven frames, five from the Rho-1, five from the Rho-2, and only one from the Rho-3 run as it gives identical results as the Rho-2 run. For these frames, we compute the TDDFT excitations using as quantum region either the chromophore (RPSB) or the chromophore and the counter-ion (RPSB+Glu113), without (None) and with (Full) the MM point charges of the whole system. We also obtain the excitation energies of the M10 cluster used in the Zindo calculations. We show the CAM-B3LYP results in Table 6.2 and in Figure 6.7. When we include the effect of the protein environment, the frames from all runs display rather similar excitations even though the counter-ion distance is about 
$0.4 \AA$ longer for the Rho-1 run. Given the similarity in the excitations, we will not distinguish in the following between frames from individual runs.

Table 6.2: CAM-B3LYP/6-31+G* vertical excitations energies (eV) for frames from absorption maximum of the Rho-1, Rho-2, and Rho-3 runs.

\begin{tabular}{|c|c|c|c|c|c|c|}
\hline \multirow[t]{2}{*}{ Frame } & \multirow{2}{*}{$\begin{array}{l}\text { QM: } \\
\text { MM: }\end{array}$} & \multicolumn{2}{|c|}{ RPSB } & \multicolumn{2}{|c|}{ RPSB+Glu113 } & \multirow{2}{*}{$\begin{array}{l}\text { M10 } \\
\text { None }\end{array}$} \\
\hline & & None & Full & None & Full & \\
\hline \multirow[t]{5}{*}{ Rho-1 } & 1 & 2.49 & 2.68 & 2.71 & 2.69 & 2.58 \\
\hline & 2 & 2.31 & 2.58 & 2.61 & 2.59 & 2.51 \\
\hline & 3 & 2.33 & 2.67 & 2.64 & 2.65 & 2.53 \\
\hline & 4 & 2.45 & 2.70 & 2.69 & 2.71 & 2.56 \\
\hline & 5 & 2.38 & 2.69 & 2.67 & 2.70 & 2.57 \\
\hline \multirow[t]{5}{*}{ Rho-2 } & 6 & 2.36 & 2.64 & 2.71 & 2.66 & 2.60 \\
\hline & 7 & 2.42 & 2.63 & 2.72 & 2.62 & 2.53 \\
\hline & 8 & 2.38 & 2.65 & 2.71 & 2.62 & 2.58 \\
\hline & 9 & 2.38 & 2.63 & 2.78 & 2.66 & 2.59 \\
\hline & 10 & 2.38 & 2.68 & 2.78 & 2.70 & 2.62 \\
\hline Rho-3 & 11 & 2.36 & 2.65 & 2.72 & 2.65 & 2.61 \\
\hline \multicolumn{2}{|c|}{ Average - Fr 1-5 } & 2.39 & 2.66 & 2.66 & 2.67 & 2.55 \\
\hline \multicolumn{2}{|c|}{ Average - Fr 6-11 } & 2.38 & 2.65 & 2.74 & 2.65 & 2.59 \\
\hline \multicolumn{2}{|c|}{ Average - Fr 1-11 } & 2.39 & 2.65 & 2.70 & 2.66 & 2.57 \\
\hline
\end{tabular}

The distorted chromophore without MM charges (RPSB/None) has an average excitation energy of $2.39 \mathrm{eV}$, which is rather close to the CAM-B3LYP value of $2.36 \mathrm{eV}$ obtained for the RPSB model optimized in the gas phase (see Table 6.3). Therefore, the increased BLA and significant distortion of the chromophore does not considerably affect the excitation of the isolated chromophore. As expected, adding the full protein environment by embedding the chromophore in a sea of MM point charges (RPSB/Full) results in a blue-shift of about $0.2-0.3 \mathrm{eV}$. A similar blue shift is obtained if the Glu113 counter-ion is added to the QM region (RPSB+Glu113/None) and the further addition of the rest of the protein as MM charges (RPSB+Glu113/Full) has almost no effect. Therefore, if the MM charges are included, very similar excitations are obtained regardless of whether the counter-ion is part of the QM region or not. Furthermore, in the excited states, we do not observe charge transfer between the chromophore and the counter-ion. Consequently, if we limit ourselves to a $\mathrm{QM} / \mathrm{MM}$ description for absorption, we do not need to include the counter-ion in the $\mathrm{QM}$ region as it role seems to be mainly electrostatic.

The CAM-B3LYP/MM excitations have an average of $2.65 \mathrm{eV}$ and are therefore blue-shifted with respect to the experimental absorption maximum of $2.49 \mathrm{eV}$. When going from the QM/MM description to the M10 cluster model, we obtain a red shift which ranges between 0.05 and $0.15 \mathrm{eV}$ as shown in Figure 6.7f. One may ask if the red shift obtained with the cluster model is due to spurious charge transfer 

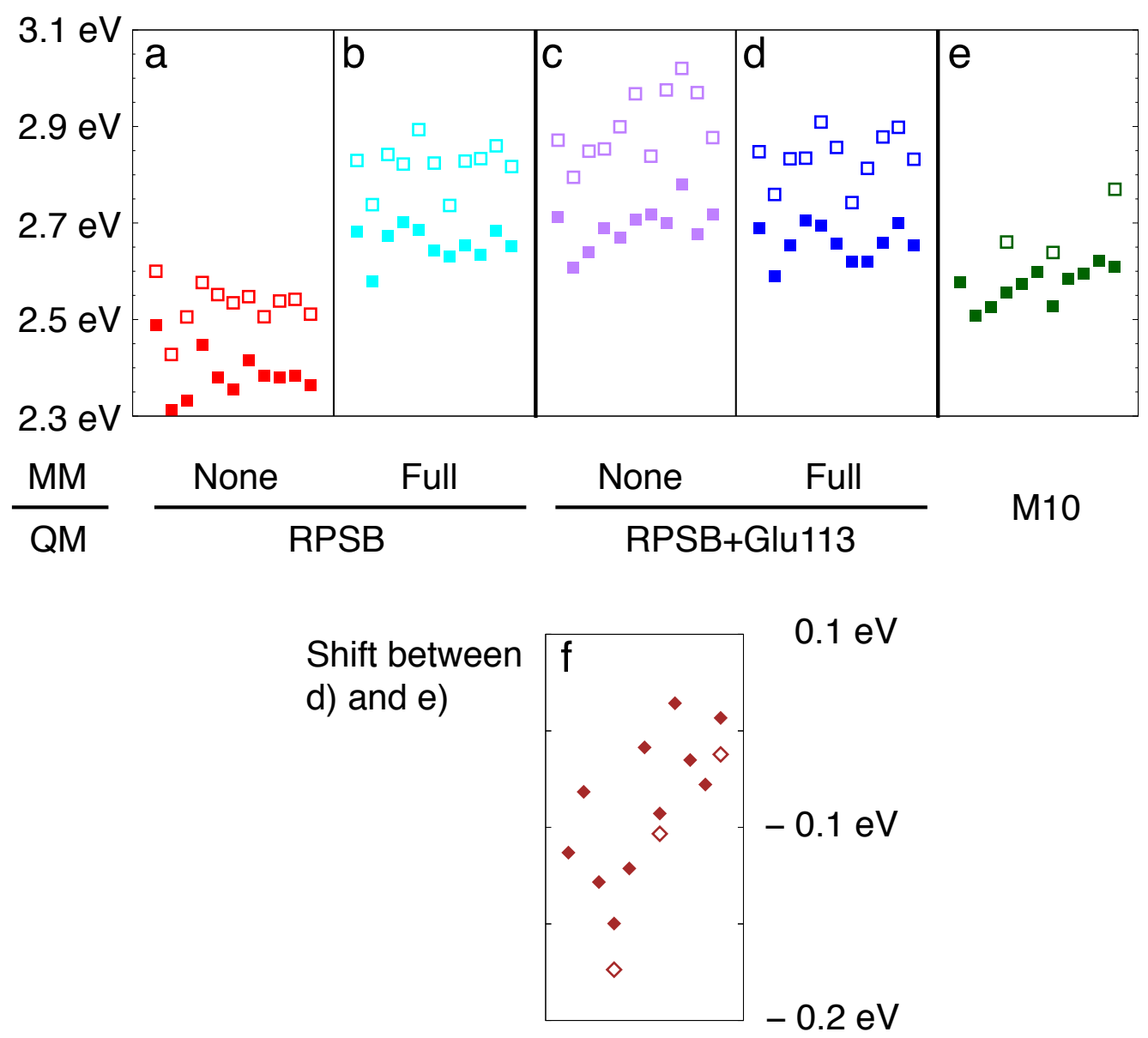

Figure 6.7: CAM-B3LYP (filled symbols) and LC- $\omega$ PBE (empty symbols) vertical excitation energies for frames from the absorption maximum of the Rho-1, Rho-2, and Rho-3 runs. We show the excitation energies computed with different QM/MM models in panels a)-d), and with the M10 cluster in panel e). In panel f), we show the shift in the excitation energies obtained when going from RPSB+Glu113/Full to the M10 cluster. The order of the frames is the same as in Table 6.2.

problems, which often plague TDDFT calculations. The CAM-B3LYP functional is however from the class of long-range corrected DFT functionals designed to mend this kind of problems. In fact, we observe no indication of spurious intermolecular charge transfer and, also for the large M10 cluster, the character of the excitation has a dominant $\pi \rightarrow \pi^{*}$ contribution on the chromophore as in the QM/MM calculations. Therefore, the red shift obtained with the cluster model is not an artifact of approximate TDDFT but is due to the quantum description of the protein environment. 
In Figure 6.7, we also present TDDFT results obtained with the LC- $\omega$ PBE functional, which is also a long-range corrected functional but has $100 \%$ exact-exchange at long range as compared to 65\% in CAM-B3LYP. As well known for singlet excited states [94], the LC- $\omega$ PBE excitations are blue-shifted with respect to to CAMB3LYP values but display the same trends. In particular, as shown in Figure 6.7f, the red shifts obtained with the M10 cluster are very similar for LC- $\omega$ PBE and CAMB3LYP, confirming the validity of the CAM-B3LYP results.

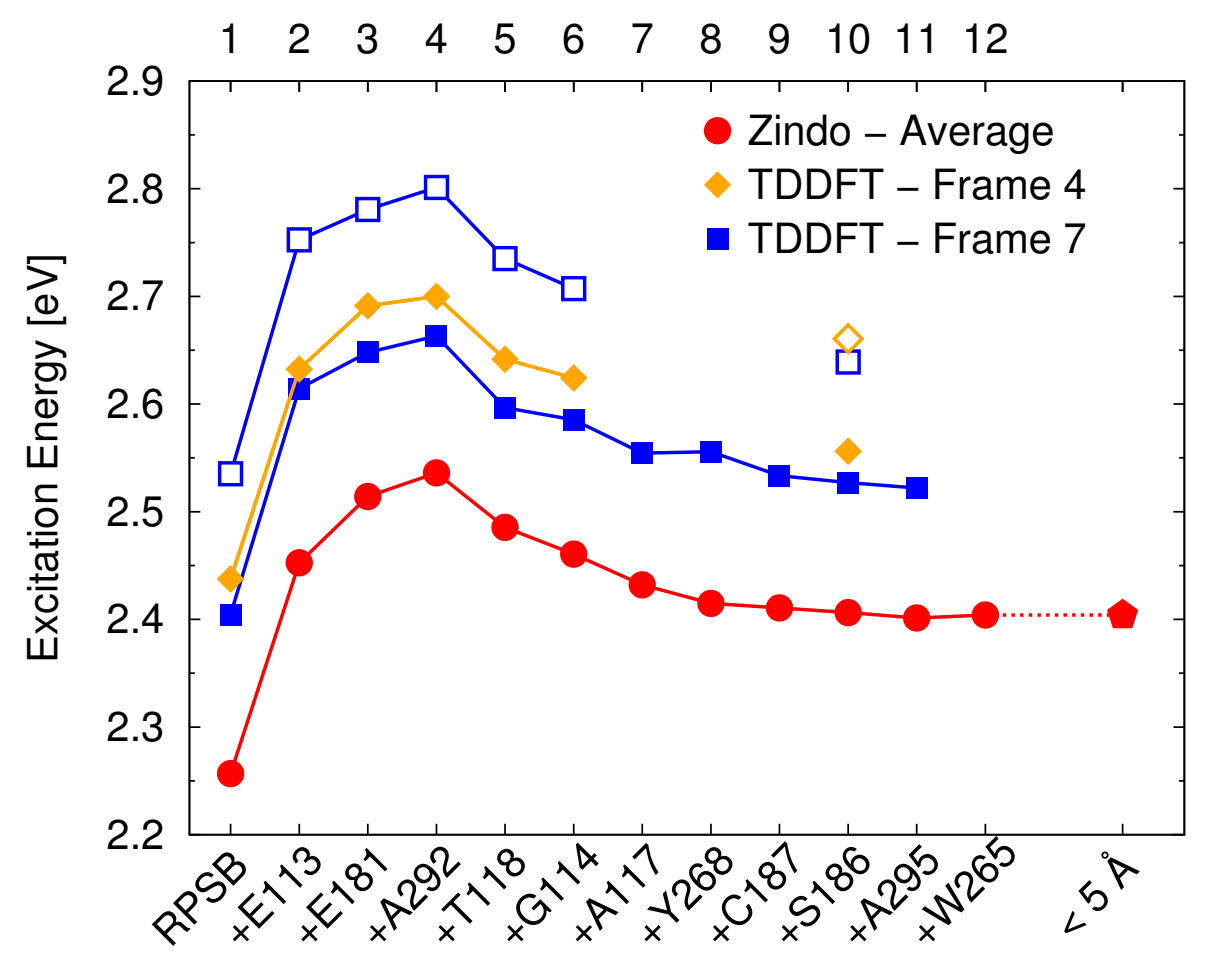

Figure 6.8: CAM-B3LYP (filled symbols) and LC- $\omega$ PBE (open symbols) excitation energies computed on different cluster models extracted from frames 4 and 7.

In Figure 6.8, we show the CAM-B3LYP and LC- $\omega$ PBE excitations obtained on the different cluster models extracted from frames 4 and 7 . For both frames, the CAM-B3LYP excitation energies nearly parallel the average Zindo values, being systematically blue shifted. A similar behavior is observed for the LC- $\omega$ PBE values. This suggests that also the TDDFT excitation energies like the Zindo ones are converged for the M10 cluster and that we would therefore not observe further changes by including extra residues. 


\subsubsection{Highly-Correlated Excitations}

Table 6.3: Vertical excitation energies of the 11-cis RPSB in the gas-phase computed on DFT geometries. These results are from our previous study in Ref. 59 and are presented for comparison with the values obtained in the protein.

\begin{tabular}{lccc}
\hline & \multicolumn{3}{c}{ Geometry } \\
\cline { 2 - 4 } & BLYP (0\%) & B3LYP (20\%) & M06-2X (54\%) \\
\hline BLA & 0.024 & 0.033 & 0.051 \\
$d_{\text {C6-N16 }}$ & 11.73 & 11.62 & 11.52 \\
$\phi_{\text {C5-C6-C7-C8 }}$ & -29.7 & -33.5 & -38.0 \\
\multicolumn{2}{l}{ Vertical excitation energies (eV) } & & \\
CAM-B3LYP & 2.36 & 2.43 & 2.49 \\
LC- $\omega$ PBE & 2.39 & 2.48 & 2.61 \\
CASPT2 & 2.12 & 2.20 & 2.30 \\
NEVPT2 & 2.18 & 2.26 & 2.33 \\
DMC & $2.22(4)$ & $2.37(3)$ & - \\
\hline
\end{tabular}

To compute the excitation energies with various highly-correlated methods, we consider a subset of only three frames from the eleven frames from the previous section, that is, frame 4 from Rho-1, frame 7 from Rho-2, and frame 11 from Rho-3. For these frames, we perform calculations with the approximate singles and doubles coupled-cluster (CC2) method, two flavors of multi-configuration perturbation theory (CASPT2) characterized by two different zero-order Hamiltonians, and the more recent and advanced $n$-electron valence state perturbation theory (NEVPT2). All these perturbative calculations are based on the same zero-order complete-activespace self-consistent-field (CASSCF) wave functions. Finally, we also employ the diffusion Monte Carlo (DMC) approach, which has been shown to perform well in describing excited states. We note that we recently demonstrated that the more recent flavor of CASPT2, NEVPT2, and DMC methods agree rather well for the excitation energies of the chromophore in the gas phase [59], as also shown in Table 6.3.

The TDDFT results obtained in the previous section indicate that going beyond the conventional QM/MM description with the use of large quantum clusters changes the excitation energies by a non-negligible amount. Unfortunately, while it is possible to use large quantum models within TDDFT, a system of 250 atoms is out of the question for most highly-correlated methods. Therefore, for the calculations with the high-level methods, we will remain within a QM/MM scheme and compare with the corresponding TDDFT/MM values in Table 6.4.

The CASPT2 excitations for the chromophore embedded in the MM charges (RPSB/Full) are very comparable to the CAM-B3LYP excitations, and thus blue shifted with respect to the experimental value. The behavior of the two approaches 
Table 6.4: Vertical excitation energies $(\mathrm{eV})$ on selected frames from the absorption maximum.

\begin{tabular}{|c|c|c|c|c|c|c|c|}
\hline \multirow[t]{2}{*}{ Method } & \multirow[t]{2}{*}{ Fr. } & \multirow{2}{*}{$\begin{array}{l}\text { QM: } \\
\text { MM: }\end{array}$} & \multicolumn{2}{|c|}{ RPSB } & \multicolumn{2}{|c|}{ RPSB+Glu113 } & \multirow{2}{*}{$\begin{array}{l}\text { M10 } \\
\text { None }\end{array}$} \\
\hline & & & None & Full & None & Full & \\
\hline \multirow[t]{3}{*}{ CAM-B3LYP $^{a}$} & 4 & & 2.45 & 2.70 & 2.69 & 2.71 & 2.56 \\
\hline & 7 & & 2.42 & 2.63 & 2.72 & 2.62 & 2.53 \\
\hline & 11 & & 2.36 & 2.65 & 2.72 & 2.65 & 2.61 \\
\hline \multirow[t]{3}{*}{ LC- $\omega \mathrm{PBE}^{a}$} & 4 & & 2.57 & 2.82 & 2.85 & 2.83 & 2.66 \\
\hline & 7 & & 2.55 & 2.74 & 2.84 & 2.74 & 2.64 \\
\hline & 11 & & 2.51 & 2.82 & 2.88 & 2.83 & 2.77 \\
\hline \multirow[t]{3}{*}{$\mathrm{CC} 2^{b}$} & 4 & & 2.03 & 2.59 & & & \\
\hline & 7 & & 2.08 & 2.49 & & & \\
\hline & 11 & & 2.16 & 2.54 & & & \\
\hline \multirow[t]{3}{*}{ CASPT $^{c}$} & 4 & & 2.29 & 2.71 & $2.78^{f, g}$ & 2.75 & \\
\hline & 7 & & 2.22 & 2.61 & $2.80^{f}$ & 2.66 & \\
\hline & 11 & & 2.16 & 2.72 & $2.89^{f}$ & $2.80^{f}$ & \\
\hline \multirow[t]{3}{*}{ NEVPT $^{d}$} & 4 & & 2.39 & 2.76 & & & \\
\hline & 7 & & 2.35 & 2.65 & & & \\
\hline & 11 & & 2.28 & 2.74 & & & \\
\hline \multirow[t]{3}{*}{$\mathrm{DMC}^{e}$} & 4 & & $2.52(3)$ & $2.78(3)$ & & & \\
\hline & 7 & & & $2.73(3)$ & & & \\
\hline & 11 & & & $2.82(3)$ & & & \\
\hline \multicolumn{7}{|c|}{$\begin{array}{l}{ }^{a} \text { 6-31+G* basis set; }{ }^{b} \text { ANO-L-VDZP basis set; } \\
{ }^{c} \text { SS-CASPT2 using ANO-L-VDZP and CAS }(12,13) \text { over } 2 \text { states; } \\
{ }^{d} \text { NEVPT2/SC using ANO-L-VDZP and CAS }(12,13) \text { over } 2 \text { states; } \\
\text { NE } \text { DMC using D+ basis and CAS }(12,12) \text { over } 2 \text { states: }\end{array}$} & \\
\hline
\end{tabular}

is however not the same since the CASPT2 method responds much more strongly to the addition of the MM point charges: Adding the MM point charges to the isolated chromophore (RPSB/None) results in a $0.4-0.6 \mathrm{eV}$ blue shift for CASPT2, compared to only $0.2-0.3 \mathrm{eV}$ for CAM-B3LYP. As in the TDDFT calculations, the addition of the counter-ion to the QM region has very little effect on the CASPT2 excitation energies once the MM charges are included. If we consider the NEVPT2 results, we observe a small difference of $0.1 \mathrm{eV}$ between NEVPT2 and CASPT2 values for the isolated chromophore but, once the MM charges are included, the two methods yield results in very good agreement, with NEVPT2 being at most 0.05 $\mathrm{eV}$ higher. The DMC results also agree rather well with the CASPT2 and NEVTP2 estimates, and are at most $0.1 \mathrm{eV}$ higher. A similar good agreement was observed for the retinal chromophore in the gas-phase [59] (see also Table 6.3). We note that the NEVPT2 approach is known to be sensitive to the quality of the zero-order wave 
function and that can give unsatisfactory performance if the correction due to dynamical correlation is large [95]. However, for the results presented here, this is not an issue. Finally, the CC2 excitations energies (RPSB/Full) of about 2.5-2.6 eV are at variance with all other methods and in apparent agreement with the experimental absorption maximum at $2.49 \mathrm{eV}$.

The CASPT2 approach has been extensively used in last decade to study the absorption properties of Rhodopsin and other visual opsins [53-58]. Our results differ in the use of a more recent and improved formulation of the CASPT2 theory, as we will discuss in more detail below. Employing the older formulation of the CASPT2 method as used in previous Rhodopsin studies results in excitations significantly red shifted by $0.3 \mathrm{eV}$, in the range of 2.3-2.4 eV. A comparison of the two flavors of this perturbative approach with other methods (NEVPT2 and DMC) with their balanced description of static and dynamical correlation strongly indicates that the improved CASPT2 formulation used here is more accurate.

In summary, we observe that TDDFT/CAM-B3LYP, CASPT2, NEVPT2, and DMC yield very comparable results within the conventional QM/MM scheme with the chromophore in the QM region and the rest of the protein treated as MM point charges. The results obtained are $0.2-0.3 \mathrm{eV}$ blue shifted as compared to the experimental absorption maximum at $2.49 \mathrm{eV}$. We stress again that, even though an enlarged quantum region brings the TDDFT energies in better agreement with the experimental value, a calculation with the same large QM region is not possible with the other methods. Nevertheless, it is plausible that a similar (or larger, given the stronger response of the high-level methods to the MM charges) variation in the excitation energies would be obtained for a calculation with these highly-correlated methods and the same large QM cluster.

\subsubsection{Comparison with Previous CASPT2/MM Studies}

As briefly mentioned in the previous Section, the CASPT2 approach in its older formulation has been the method of choice for the investigation of the absorption of Rhodopsin [53-58] in the last decade. These studies have employed a conventional $\mathrm{QM} / \mathrm{MM}$ scheme and generally reported a remarkable agreement with the experimental absorption maximum, yielding results in a range of 2.3 to $2.6 \mathrm{eV}$. This must be contrasted with our findings of significantly blue shifted excitations computed with highly-correlated approaches and an MM environment. Here, we will show that the agreement of previous studies appears to be fortuitous and that the reported results depend very much on the precise details of the computational procedure employed.

The older formulation of the CASPT2 approach (CASPT2/0-IPEA) is based on a different zero-order Hamiltonian than the one used in this work. The newer formulation of the CASPT2 approach (CASPT2/S-IPEA) we use employs an improved zero-order Hamiltonian, the so-called IPEA Hamiltonian, which was developed to give on average more accurate results [84]. In our previous work on gas-phase retinal $[59,96]$, we have shown that CASPT2/S-IPEA gives more accurate excitations 
in agreement with NEVPT2 and DMC methods, while the CASPT2/0-IPEA excitation energies are at variance and systematically lower by $0.3 \mathrm{eV}$. The exact same behavior is observed in the previous section for the Rhodopsin frames.

To model Rhodopsin, previous CASPT2/MM studies have used the available crystallographic structures where only the geometry of the chromophore is optimized with the low-correlation CASSCF method, which largely lacks the inclusion of dynamical correlation. As we have shown for the RPSB in the gas phase, the CASSCF method leads to an inadequate description for the structural model of retinal and yields excitation energies that are $0.3 \mathrm{eV}$ higher than those obtained with more appropriate DFT structural models $[59,97]$. Another important difference with our work is that previous CASPT2/MM studies have used very different protonation states, especially in considering the Glu181 residues to be protonated (neutral). Finally, only the chromophore was included in the QM region while the rest of the environment has been described by using Amber MM point charges, and no thermal effects were included.

To further understand the discrepancy with previous CASPT2/MM studies, we follow two routes. In the first route, we employ exactly the same structural model as used in Refs. 57 and 58, which was constructed from the 1U19 [17] crystallographic structure (the model was kindly provided by one of the authors of Ref. 58, N. Ferré). From this model, we also construct different structures either by changing the protonotation state of the Glu181 residue or by reoptimizing the orginal CASSCF chromophore with DFT and the BLYP functional. In the second route, we also start from the $1 \mathrm{U} 19$ crystallographic structure but protonate all residues as in our model. We then keep the protein environment fixed and optimize the geometry of the chromophore, with either CASSCF or DFT/BLYP.

Table 6.5: CASPT2/MM excitation energies (eV) computed with the Rhodopsin (1U19) CASSCF/Amber model used in Refs. 57 and 58. We note that Glu181 is protonated in this model.

\begin{tabular}{llc} 
CASPT2 & Basis & Excitation \\
\hline 0-IPEA & 6-31G* & $\mathbf{2 . 3 8}$ \\
& ANO-L-VDZP & 2.22 \\
S-IPEA & 6-31G* & 2.75 \\
& ANO-L-VDZP & $\mathbf{2 . 6 3}$ \\
\hline
\end{tabular}


In Table 6.5, we present the CASPT2/MM excitation energies computed for the original structure employed in Refs. 57 and 58, with different zero-order Hamiltonians and basis sets. For the older CASPT2/0-IPEA formulation, we only obtain an excitation compatible with the experimental absorption maximum if we use the small and unconverged $6-31 \mathrm{G}^{*}$ basis set as normally done in these older studies $[57,58]$, while a better ANO-L-VDZP basis leads to a value which is too low. The CASPT2/S-IPEA formulation gives excitations systematically blue-shifted by about $0.4 \mathrm{eV}$. Clearly, we would be leaving the realm of ab initio calculations if we were to adopt the point of view that certain approaches should be used in combination with particular basis sets as it has been claimed in a recent Rhodopsin study [58]. Here, we instead conclude that the apparent agreement of the CASPT2/0-IPEA formulation with experiments is due to a favorable cancelation of errors.

Table 6.6: CASPT2/MM excitations (eV) obtained from different Rhodopsin (1U19) models. The chromophore is optimized with either CASSCF or BLYP, using the protonation state of Glu181 as indicated.

\begin{tabular}{lllc} 
Model & Geometry & Glu181 & CASPT2/S-IPEA \\
\hline From Refs. 57 and 58 & CASSCF & Prot. & $\mathbf{2 . 6 3}$ \\
& CASSCF & Deprot. & 2.96 \\
& BLYP & Prot. & 2.17 \\
& BLYP & Deprot. & $\mathbf{2 . 5 5}$ \\
From present work & CASSCF & Deprot. & 3.14 \\
& BLYP & Deprot. & $\mathbf{2 . 5 7}$ \\
\hline
\end{tabular}

In Table 6.6, we consider different models constructed with different possible combinations of the protonation state of Glu181 and methods used to optimize the chromophore. This game leads to the interesting finding that different models may accidentally lead to final excitation energies which are rather close. However, the corresponding structures are in fact rather different as regards the geometry of the chromophore and the chemical environment of the protein. Moreover, even though the final excitation energies from the CASSCF model from Refs. 57 and 58 and our B3LYP protein are equivalent, the conclusions on the effect of the environment are rather different. The former model leads to a counter-ion quenching effect from the rest of the protein of almost $0.9 \mathrm{eV}$ while the latter is characterized by a corresponding value of only $0.1 \mathrm{eV}$. 


\subsection{Discussion and Conclusions}

In this work, we have performed a careful and thorough investigation of the absorption properties of Rhodopsin with a wide range of theoretical methods. We carefully constructed a model of Rhodopsin embedded in a realistic membrane environment, which was extensively equilibrated at room temperature at the classical MD level. Starting from an unbiased initial structure selected with the help of clustering analysis, we performed QM/MM MD simulations longer than $10 \mathrm{ps}$ at room temperature to obtain an accurate geometrical description of the retinal chromophore and of the thermal fluctuations of the chromophore-protein system. The hydrogen bond network around the chromophore was very stable throughout the classical and the QM/MM MD simulation, which is an additional indication of the robustness of our model. With the QM/MM trajectories, we have computed the Zindo absorption spectrum of Rhodopsin at room temperature, which we then used to identify configurations from the phase space close to the Zindo absorption maximum.

For these selected structures, we computed the excitation energies with a variety of ab initio approaches (TDDFT, multi-reference perturbation, and quantum Monte Carlo methods) in combination with different schemes to model the chromphoreprotein interaction. Within the conventional QM/MM scheme of a small quantum region (the chromophore with at most the counter-ion) embedded in classical point charges, we obtained excitation energies at the TDDFT and highly-correlated level that are $0.2-0.3 \mathrm{eV}$ higher then the experimental absorption maximum at $2.49 \mathrm{eV}$. The use of a larger quantum region (about 250 atoms) shifts the TDDFT excitations to lower energies, and brings them closer to the experimental absorption maximum. As for the many previous multi-reference perturbation (CASPT2) studies of Rhodopsin in a classical environment [53-58], claiming a remarkable agreement with experiments, we demonstrated that this agreement is fortuitous and due to a favorable cancelation of errors for the use of a superseded flavor of perturbative approach and an incorrect protein model. Finally, we showed that, when only one structure is used (no thermal sampling), it is not difficult to obtain the correct answer (the experimental absorption maximum) for the wrong reasons thanks to the many parameters (e.g. protonation states, geometry, excited-state method, embedding model etc.) one can play with in the description of a system as complex as Rhodopsin.

An important finding of our study is that the description of the protein in terms of classical, non-polarizable point charges as in conventional QM/MM schemes appears to be inadequate to describe the absorption of Rhodopsin. In the past, other authors had reached similar conclusions [40-42] but these older studies were not as definite as ours since they either lacked the inclusion of thermal fluctuations (they used only one structure for the protein) or did not employ such a large range of theoretical methods to validate their conclusions. Our study is instead rather extensive and strongly points to the need of a better environmental description in Rhodopsin. Perhaps, this should not come as a surprise since the excitation involves a rather large transfer of positive charge from the protonated Schiff base towards the $\beta$ - 
ionone ring, and a large difference in dipole moment between the ground and the excited state, estimated to be about $12 \mathrm{D}$ for RPSB in solution [18]. Therefore, such a change upon photo-excitation should induce a response (polarization) of the protein environment, an effect not taken into account by the fixed MM point charges used in the conventional QM/MM scheme.

Here, we provided a better description of the environment by enlarging the quantum region to include as many as 250 atoms. Obviously, this solution is not optimal since these calculations are quite expensive already at the TDDFT level, and are prohibitive for the high-level excited-state methods we consider reliable to describe retinal absorption. However, not all hope is lost. Another important finding in our TDDFT calculations with large clusters is that the excitations in Rhodopsin do not display intermolecular charge transfer but remain confined to the chromophore. Therefore, a multiscale partition of the system in an active region and a surrounding protein is still possible, and one might obtain more accurate excitation energies with a better embedding model of a polarizable protein environment.

Even though we cannot employ such an enlarged QM region with the highlycorrelated excited-state methods, it is quite plausible that their behavior is rather similar to the TDDFT one since, with a MM description of the protein, the TDDFT excitation energies are very close to the multi-reference perturbation and quantum Monte Carlo values. Therefore, if we assume that a larger QM region red-shifts also the highly-correlated excitation energies by the same amount, the resulting estimates are in the range of $2.6-2.7 \mathrm{eV}$, that is, about $0.1-0.2 \mathrm{eV}$ higher than the experimental absorption maximum at $2.49 \mathrm{eV}$. This observation leads us to another important question, which has been totally overlooked in previous ab initio studies of Rhodopsin: Should we compare the vertical excitation energy with the location of the experimental absorption maximum of Rhodopsin as commonly done in previous theoretical studies? If we look at the experimental absorption spectrum, it is not clear that this is the correct way to proceed.

In Figure 6.9, we show the experimental absorption spectrum of Rhodopsin obtained at room $(293 \mathrm{~K})$ and low $(10 \mathrm{~K})$ temperature [31]. In both cases, the absorption spectrum is very broad and unstructured with a large full width at half maximum of about $0.5 \mathrm{eV}$. Furthermore, the intensity is still about $90 \%$ if we move $0.1 \mathrm{eV}$ in either direction from the maximum. Such a spectral shape can be due to a combination of homogeneous and inhomogeneous broadening [24, 25]. Homogeneous broadening is defined as due to all mechanisms that broaden the spectrum of a single chromophore (e.g. the lifetime of the excitation) while inhomogeneous broadening is due to ensemble effects (e.g. fluctuations in the structure) that result in different excitations for different chromophores. Numerous experimental studies have investigated the absorption properties of Rhodopsin and Bacteriorhodopsin [23-30,32] with for instance resonance Raman spectroscopy [23-27] or spectral hole burning [27-29]. The picture that emerges from these studies is that the broadening can be partly explained with strong vibronic effects related to the torsional mode around the active $\mathrm{C}_{11}-\mathrm{C}_{12}$ bond in photoisomerization $[25,28,32]$ and low-frequency torsional modes on the chromophore $[25,26]$. These vibronic bands are further broadened by 


\section{a)}

\section{Room Temp. $(T=293 \mathrm{~K})$}

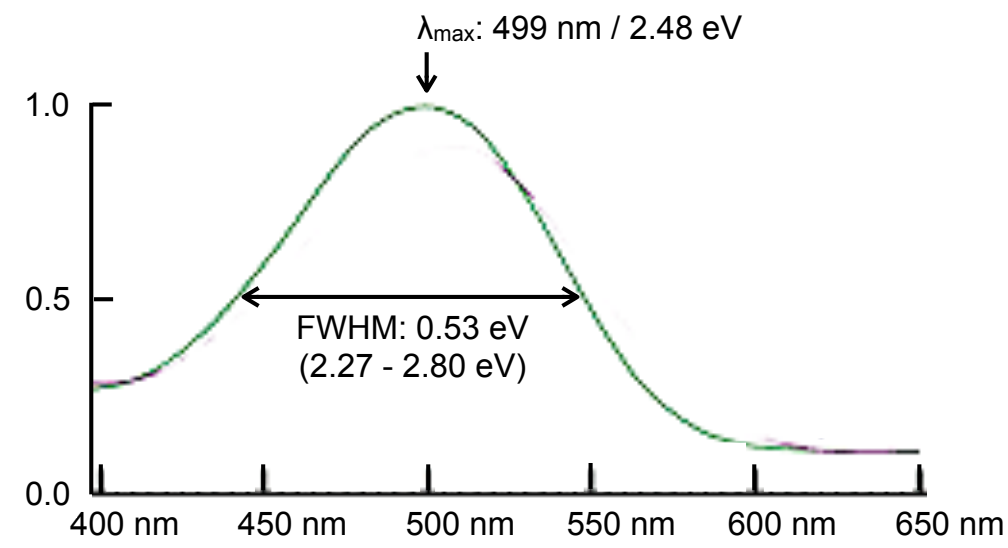

b)

Low Temp. $(\mathrm{T}=10 \mathrm{~K})$

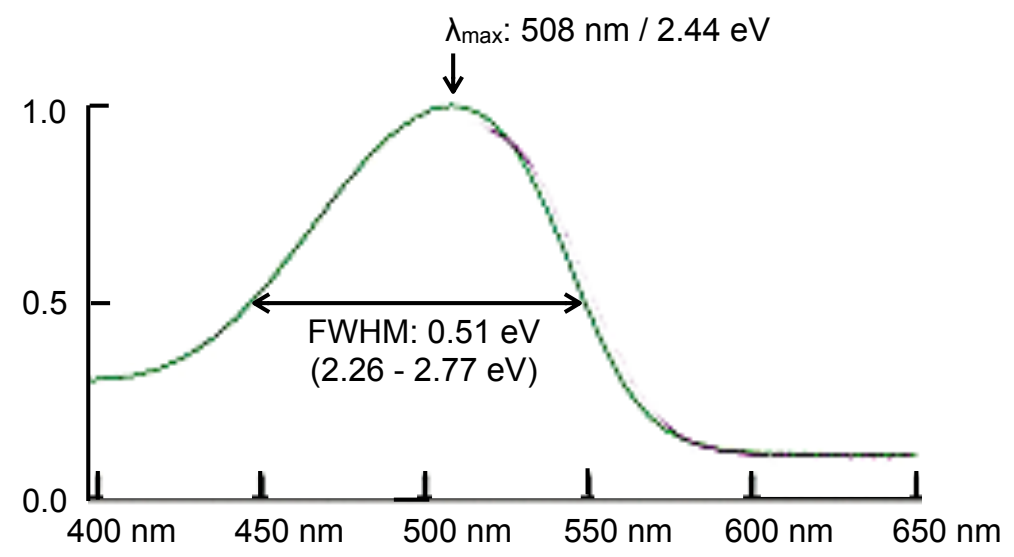

Figure 6.9: The experimental absorption spectrum of Rhodopsin obtained at a) room temperature $(\mathrm{T}=293 \mathrm{~K})$, and $\mathrm{b})$ low temperature $(\mathrm{T}=10 \mathrm{~K})$. Figures adapted from Ref. 31.

considerable inhomogeneous (and other homogeneous effects), which persist at low temperature and result in a featureless absorption spectrum. Therefore, a remaining disagreement of $0.1-0.2 \mathrm{eV}$ between the theoretical vertical excitation and the location of the absorption maximum in the broad experimental spectrum can well be expected.

In conclusion, our calculations identify the necessary ingredients for an accurate description of the absorption of Rhodopsin. We demonstrate the need of a realistic structural model of the system obtained through extensive QM/MM molecular dynamics simulations, which account for the thermal fluctuations in the chromophoreprotein structure and ensure an accurate description of the chromophore. To estimate 
the absorption maximum, it is important to employ not one but several configurations of the system which are representative of the relevant region of phase space. In addition, the use of a single structure may hide the limitations of the model, especially if this one structure happens to yield a plausible excitation energy as in many previous studies of Rhodopsin. Finally, a proper description of the environment as given by a larger quantum region is crucial in the computation of the excitation energies of Rhodopsin since the conventional QM/MM model is inadequate.

\subsection{Bibliography}

[1] K. Palczewski, Annu. Rev. Biochem. 75, 743 (2006).

[2] S. O. Smith, Annu. Rev. Biophys. 39, 309 (2010).

[3] T. Ebrey and Y. Koutalos, Prog. Retinal Eye Res. 20, 49 (2001).

[4] B. Nickle and P. R. Robinson, Cell. Mol. Life. Sci. 64, 2917 (2007).

[5] J. Partridge and W. De Grip, Vision Res. 31, 619 (1991).

[6] Y. Shichida and H. Imai, Cell. Mol. Life. Sci. 54, 1299 (1998).

[7] R. Stenkamp, S. Filipek, C. Driessen, D. Teller, and K. Palczewski, Biochim. Biophys. Acta 1565, 168 (2002).

[8] R. E. Stenkamp, D. C. Teller, and K. Palczewski, Arch. Pharm. Pharm. Med. Chem. 338, 209 (2005).

[9] Y. Shichida and T. Morizumi, Photochem. Photobiol. 83, 70 (2007).

[10] M. B. Nielsen, Chem. Soc. Rev. 38, 913 (2009).

[11] Y. Shichida and T. Matsuyama, Phil. Trans. R. Soc. B 364, 2881 (2009).

[12] K. Tsutsui and Y. Shichida, Photochem. Photobiol. Sci. 9, 1426 (2010).

[13] M. F. Brown, G. F. Salgado, and A. V. Struts, Biochim. Biophys. Acta 1798, 177 (2010).

[14] K. Palczewski, Science 289, 739 (2000).

[15] D. C. Teller, T. Okada, C. A. Behnke, K. Palczewski, and R. E. Stenkamp, Biochemistry 40, 7761 (2001).

[16] T. Okada, Y. Fujiyoshi, M. Silow, J. Navarro, E. M. Landau, and Y. Shichida, Proc. Natl. Acad. Sci. U.S.A. 99, 5982 (2002).

[17] T. Okada, M. Sugihara, A.-N. Bondar, M. Elstner, P. Entel, and V. Buss, J. Mol. Biol. 342, 571 (2004). 
[18] R. Mathies and L. Stryer, Proc. Natl. Acad. Sci. U.S.A. 73, 2169 (1976).

[19] K. Fahmy, F. Jäger, M. Beck, T. A. Zvyaga, T. P. Sakmar, and F. Siebert, Proc. Natl. Acad. Sci. U.S.A. 90, 10206 (1993).

[20] N. al Jandal, G. J. Farrar, A.-S. Kiang, M. M. Humphries, N. Bannon, J. B. Findlay, P. Humphries, and P. F. Kenna, Hum. Mutat. 13, 75 (1999).

[21] E. Ramon, L. J. del Valle, and P. Garriga, J. Biol. Chem. 278, 6427 (2002).

[22] J. M. Janz and D. L. Farrens, J. Biol. Chem. 279, 55886 (2004).

[23] A. B. Myers, R. A. Harris, and R. A. Mathies, J. Chem. Phys. 79, 603 (1983).

[24] A. Myers and R. Mathies, in Biological Applications of Raman Spectroscopy: VOLUME 2: Resonance Raman Spectra of Polyenes and Aromatics, edited by T. Spiro (John Wiley \& Sons, Inc., 1987), pp. 1-58.

[25] G. Loppnow and R. Mathies, Biophys. J. 54, 35 (1988).

[26] J. E. Kim, M. J. Tauber, and R. A. Mathies, Biophys. J. 84, 2492 (2003).

[27] G. R. Loppnow, R. A. Mathies, T. R. Middendorf, D. S. Gottfried, and S. G. Boxer, J. Phys. Chem. 96, 737 (1992).

[28] I.-J. Lee, J. Gillie, and C. K. Johnson, Chem. Phys. Lett. 156, 227 (1989).

[29] V. F. Kamalov, T. M. Masciangioli, and M. A. El-Sayed, J. Chem. Phys. 100, 2762 (1996).

[30] S. Haacke, S. Vinzani, S. Schenkl, and M. Chergui, ChemPhysChem 2, 310 (2001).

[31] M. N. Sandberg, T. L. Amora, L. S. Ramos, M.-H. Chen, B. E. Knox, and R. R. Birge, J. Am. Chem. Soc. 133, 2808 (2011).

[32] R. R. Birge, D. F. Bocian, and L. M. Hubbard, J. Am. Chem. Soc. 104, 1196 (1982).

[33] U. F. Röhrig, L. Guidoni, and U. Rothlisberger, ChemPhysChem 6, 1836 (2005).

[34] S. Sekharan, M. Sugihara, and V. Buss, Angew. Chem. Int. Ed. 46, 269 (2007).

[35] K. Bravaya, A. Bochenkova, A. Granovsky, and A. Nemukhin, J. Am. Chem. Soc. 129, 13035 (2007).

[36] A. Altun, S. Yokoyama, and K. Morokuma, J. Phys. Chem. B 112, 16883 (2008). 
[37] J.-y. Hasegawa, K. J. Fujimoto, and H. Nakatsuji, ChemPhysChem 12, 3106 (2011).

[38] S. Sekharan and K. Morokuma, J. Am. Chem. Soc. 133, 19052 (2011).

[39] V. R. I. Kaila, R. Send, and D. Sundholm, J. Phys. Chem. B 116, 2249 (2012).

[40] A. Warshel and Z. T. Chu, J. Phys. Chem. B 105, 9857 (2001).

[41] M. Wanko, M. Hoffmann, P. Strodel, A. Koslowski, W. Thiel, F. Neese, T. Frauenheim, and M. Elstner, J. Phys. Chem. B 109, 3606 (2005).

[42] H. Houjou, Y. Inoue, and M. Sakurai, J. Phys. Chem. B 105, 867 (2001).

[43] A. Matsuura, H. Sato, H. Houjou, S. Saito, T. Hayashi, and M. Sakurai, J. Comput. Chem. 27, 1623 (2006).

[44] M. Wanko, M. Hoffmann, T. Frauenheim, and M. Elstner, J. Phys. Chem. B 112, 11462 (2008).

[45] M. Wanko, M. Hoffmann, J. Frähmcke, T. Frauenheim, and M. Elstner, J. Phys. Chem. B 112, 11468 (2008).

[46] P. Söderhjelm, C. Husberg, A. Strambi, M. Olivucci, and U. Ryde, J. Chem. Theory Comput. 5, 649 (2009).

[47] H. C. Watanabe, Y. Mori, T. Tada, S. Yokoyama, and T. Yamato, BIOPHYSICS 6, 67 (2010).

[48] R. Rajamani, Y.-L. Lin, and J. Gao, J. Comput. Chem. 32, 854 (2011).

[49] E. W. Hernandez-Rodriguez, E. Sanchez-Garcia, R. Crespo-Otero, A. L. Montero-Alejo, L. A. Montero, and W. Thiel, J. Phys. Chem. B 116, 1060 (2012).

[50] M. Hoffmann, M. Wanko, P. Strodel, P. H. König, T. Frauenheim, K. Schulten, W. Thiel, E. Tajkhorshid, and M. Elstner, J. Am. Chem. Soc. 128, 10808 (2006).

[51] J. S. Frähmcke, M. Wanko, P. Phatak, M. A. Mroginski, and M. Elstner, J. Phys. Chem. B 114, 11338 (2010).

[52] J. S. Frähmcke, M. Wanko, and M. Elstner, J. Phys. Chem. B 116, 3313 (2012).

[53] T. Andruniów, N. Ferré, and M. Olivucci, Proc. Natl. Acad. Sci. U.S.A. 101, 17908 (2004).

[54] P. B. Coto, A. Strambi, N. Ferré, and M. Olivucci, Proc. Natl. Acad. Sci. U.S.A. 103, 17154 (2006). 
[55] T. Andruniów and M. Olivucci, J. Chem. Theory Comput. 5, 3096 (2009).

[56] G. Tomasello, G. Olaso-González, P. Altoeà, M. Stenta, L. Serrano-Andreás, M. Merchaán, G. Orlandi, A. Bottoni, and M. Garavelli, J. Am. Chem. Soc. 131, 5172 (2009).

[57] A. Strambi, P. B. Coto, N. Ferré, and M. Olivucci, Theor. Chem. Acc. 118, 185 (2007).

[58] F. Melaccio, M. Olivucci, R. Lindh, and N. Ferré, Int. J. Quantum Chem. 111, 3339 (2011).

[59] O. Valsson, C. Angeli, and C. Filippi, Phys. Chem. Chem. Phys. 14, 11015 (2012).

[60] M. Neri, S. Vanni, I. Tavernelli, and U. Rothlisberger, Biochemistry 49, 4827 (2010).

[61] W. L. Jorgensen, J. Chandrasekhar, J. D. Madura, R. W. Impey, and M. L. Klein, J. Chem. Phys. 79, 926 (1983).

[62] J. Wang, P. Cieplak, and P. A. Kollman, J. Comput. Chem. 21, 1049 (2000).

[63] U. F. Röhrig, L. Guidoni, and U. Rothlisberger, Biochemistry 41, 10799 (2002).

[64] X. Daura, K. Gademann, B. Jaun, D. Seebach, W. van Gunsteren, and A. Mark, Angew. Chem. Int. Ed. 38, 236 (1999).

[65] Released under GPL license and freely available at the URL http://www.cp2k.org.

[66] J. VandeVondele, M. Krack, F. Mohamed, M. Parrinello, T. Chassaing, and J. Hutter, Comput. Phys. Commun. 167, 103 (2005).

[67] T. Laino, F. Mohamed, A. Laio, and M. Parrinello, J. Chem. Theory Comput. 1, 1176 (2005).

[68] A. Laio, J. VandeVondele, and U. Rothlisberger, J. Chem. Phys. 116, 6941 (2002).

[69] J. P. Perdew, K. Burke, and M. Ernzerhof, Phys. Rev. Letters 77, 3865 (1996).

[70] A. Schäfer, C. Huber, and R. Ahlrichs, J. Chem. Phys. 100, 5829 (1994).

[71] S. Goedecker, M. Teter, and J. Hutter, Phys. Rev. B 54, 1703 (1996).

[72] C. Hartwigsen, S. Goedecker, and J. Hutter, Physical Review B 58, 3641 (1998). 
[73] G. Bussi, D. Donadio, and M. Parrinello, J. Chem. Phys. 126, 014101 (2007).

[74] M. J. Frisch, G. W. Trucks, H. B. Schlegel, G. E. Scuseria, M. A. Robb, J. R. Cheeseman, G. Scalmani, V. Barone, B. Mennucci, G. A. Petersson, H. Nakatsuji, M. Caricato, X. Li, H. P. Hratchian, A. F. Izmaylov, J. Bloino, G. Zheng, J. L. Sonnenberg, M. Hada, M. Ehara, K. Toyota, R. Fukuda, J. Hasegawa, M. Ishida, T. Nakajima, Y. Honda, O. Kitao, H. Nakai, T. Vreven, J. A. Montgomery, Jr., J. E. Peralta, F. Ogliaro, M. Bearpark, J. J. Heyd, E. Brothers, K. N. Kudin, V. N. Staroverov, R. Kobayashi, J. Normand, K. Raghavachari, A. Rendell, J. C. Burant, S. S. Iyengar, J. Tomasi, M. Cossi, N. Rega, J. M. Millam, M. Klene, J. E. Knox, J. B. Cross, V. Bakken, C. Adamo, J. Jaramillo, R. Gomperts, R. E. Stratmann, O. Yazyev, A. J. Austin, R. Cammi, C. Pomelli, J. W. Ochterski, R. L. Martin, K. Morokuma, V. G. Zakrzewski, G. A. Voth, P. Salvador, J. J. Dannenberg, S. Dapprich, A. D. Daniels, Ö. Farkas, J. B. Foresman, J. V. Ortiz, J. Cioslowski, and D. J. Fox, Gaussian 09 Revision A.02, Gaussian Inc. Wallingford CT 2009.

[75] T. Yanai, D. P. Tew, and N. C. Handy, Chem. Phys. Lett. 393, 51 (2004).

[76] O. A. Vydrov, J. Heyd, A. V. Krukau, and G. E. Scuseria, J. Chem. Phys. 125, 074106 (2006).

[77] O. A. Vydrov and G. E. Scuseria, J. Chem. Phys. 125, 234109 (2006).

[78] TURBOMOLE V5.1 2008, a development of University of Karlsruhe and Forschungszentrum Karlsruhe GmbH, 1989-2007, TURBOMOLE GmbH, since 2007; available from http: //www.turbomole.com.

[79] K. Eichkorn, O. Treutler, H. Öhm, M. Häser, and R. Ahlrichs, Chem. Phys. Lett. 240, 283 (1995).

[80] P. Widmark, P. Malmqvist, and B. O. Roos, Theor. Chem. Acc. 77, 291 (1990).

[81] T. H. Dunning Jr, J. Chem. Phys. 90, 1007 (1989).

[82] F. Weigend, A. Köhn, and C. Hättig, J. Chem. Phys. 116, 3175 (2002).

[83] F. Aquilante, L. De Vico, N. Ferré, G. Ghigo, P.-A. Malmqvist, P. Neogrády, T. B. Pedersen, M. Pitoňák, M. Reiher, B. O. Roos, L. Serrano-Andrés, M. Urban, V. Veryazov, and R. Lindh, J. Comput. Chem. 31, 224 (2010).

[84] G. Ghigo, B. O. Roos, and P.-Å. Malmqvist, Chem. Phys. Lett. 396, 142 (2004).

[85] N. Forsberg and P.-Å. Malmqvist, Chem. Phys. Lett. 274, 196 (1997).

[86] C. Angeli, R. Cimiraglia, S. Evangelisti, T. Leininger, and J.-P. Malrieu, J. Chem. Phys. 114, 10252 (2001). 
[87] C. Angeli, R. Cimiraglia, and J.-P. Malrieu, Chem. Phys. Lett. 350, 297 (2001).

[88] C. Angeli, R. Cimiraglia, and J.-P. Malrieu, J. Chem. Phys. 117, 9138 (2002).

[89] Neese, F. ORCA - an ab initio, Density Functional and Semiempirical program package, Version 2.8. Max-Planck-Insitut für Bioanorganische Chemie, Mülheim an der Ruhr, 2011.

[90] F. Neese, WIREs Comput Mol Sc 2, 73 (2012).

[91] CHAMP is a quantum Monte Carlo program package written by C. J. Umrigar, C. Filippi and collaborators.

[92] M. Burkatzki, C. Filippi, and M. Dolg, J. Chem. Phys. 126, 234105 (2007).

[93] M. R. Silva-Junior and W. Thiel, J. Chem. Theory Comput. 6, 1546 (2010).

[94] D. Jacquemin, V. Wathelet, E. A. Perpète, and C. Adamo, J. Chem. Theory Comput. 5, 2420 (2009).

[95] C. Angeli and M. Pastore, J. Chem. Phys. 134, 184302 (2011).

[96] O. Valsson and C. Filippi, J. Chem. Theory Comput. 6, 1275 (2010).

[97] O. Valsson and C. Filippi, J. Phys. Chem. Lett. 3, 908 (2012). 



\section{Chapter 7}

\section{Electronic Excitations of Simple Cyanine Dyes $^{\dagger}$}

The simplest cyanine dye series $\left[\mathrm{H}_{2} \mathrm{~N}(\mathrm{CH})_{n} \mathrm{NH}_{2}\right]^{+}$with $n=1,3,5,7,9$ appears to be a challenge for all theoretical excited-state methods since the experimental spectra are difficult to predict and the observed deviations cannot be easily explained with standard arguments. We compute here the lowest vertical excitation energies of these dyes using a variety of approaches, namely, complete active space second-order perturbation theory (CASPT2), quantum Monte Carlo methods (QMC), coupled cluster linear response up to third approximate order (CC3), and various flavors of timedependent density functional theory (TDDFT) including the recently proposed perturbative correction scheme (B2PLYP). In our calculations, all parameters such as basis set, active space, and geometry dependence are carefully analysed. We find that all wave function methods give reasonably close excitation energies, with CASPT2 yielding the lowest values, and that the B2PLYP scheme gives excitations in satisfactory agreement with $\mathrm{CC} 3$ and DMC, significantly improving on the generalized gradient and hybrid approximations. Finally, to resolve the remaining discrepancy between predicted excitation energies and experimental absorption spectra, we also investigate the effect of excited-state relaxation. Our results indicate that a direct comparison of the experimental absorption maxima and the theoretical vertical excitations is not possible due to the presence of non-vertical transitions. The apparent agreement of earlier CASPT2 calculations with experiments was an artifact of the choice of active space and the use of an older definition of the zero-order Hamiltonian.

\footnotetext{
${ }^{\dagger}$ This chapter has been published as R. Send, O. Valsson, and C. Filippi, "Electronic Excitations of Simple Cyanine Dyes: Reconciling Density Functional and Wave Function Methods", J. Chem. Theory Comput. 2011, 7, 444-455
} 


\subsection{Introduction}

Cyanine dyes are characterized by a conjugated $\pi$-electron system connecting two nitrogen atoms and carrying a positive charge [1]. They are naturally occurring as red colorants in fly agaric mushrooms or red beet [2], and are of great industrial interest for their application in solar cells [3], optical storage media (CDs, DVDs) [4], cancer cell recognition [5], nonlinear optics [6], and as biomarkers for nucleic acid detection [7]. This wide range of important applications has made cyanine dyes an early target of theoretical studies aimed at demonstrating the predictive power of computational approaches [8].

In the last two decades, efficient computational approaches for excited states have been developed, which allow the description of large dyes and the fast screening of molecular libraries in search of specific excited-state properties [9]. In particular, time-dependent density functional theory (TDDFT) [10-12] has become the method of choice for the study of large molecular systems, and has been successfully employed to search for highly specialized chromophores and investigate several dye families $[13,14]$. The efficiency of TDDFT comes in some cases at the price of lower accuracy as compared to conventional highly-correlated quantum chemistry methods. It is for instance well known that the description of excitations with chargetransfer, multi-reference, or Rydberg character is generally problematic in TDDFT. Since none of these features appears to characterize the lowest excited state of the cyanine dyes, one would expect TDDFT to be well suited for the description of this class of systems.

Surprisingly, as early as 2001, Schreiber et al. [15] showed that the excitation energies of the cyanine dyes obtained by TDDFT deviate by more than $1 \mathrm{eV}$ from the values obtained with the CASPT2 method, which is often regarded as one of the most accurate excited-state approaches available. Since the examples chosen in Ref. 15 were the simplest models of cyanine dyes, the result suggests that TDDFT is not applicable to any member of this dye family. Until today, none of the available density functionals significantly improves the agreement with the reference CASPT2 values given in Ref. 15. The reasons for the large errors in the TDDFT results for the cyanine dyes are not understood.

Since the early work by Schreiber et al. [15], excited-state methods have seen several important developments: (i) The efficient implementation of coupled-cluster (CC) response methods in combination with the resolution-of-the-identity (RI) approximation represents a powerful single-reference complement to TDDFT [16]; (ii) efficient excited-state gradient methods render a large number of excited-state properties accessible [17, 18]; (iii) developments in algorithms and hardware allow the use of larger basis sets and higher-level theories; (iv) Quantum Monte Carlo (QMC) methods can be used as alternative to CASPT2 and independent validation of TDDFT [19-22]; $(v)$ the CASPT2 method has been modified and generally improved by the introduction of a novel definition of the zeroth-order Hamiltonian [23].

None of these developments have been fully exploited in recent calculations of the cyanine dyes, where most efforts have instead been directed to apply different 
flavours of density functionals in order to improve the excitations and gain insight into the shortcomings of TDDFT. Unfortunately, none of the used functionals has yielded significant improvement and the insight gained has therefore been limited. The only exception is the B2PLYP scheme by Grimme, which incorporates a perturbative correction based on Kohn-Sham orbitals in a form similar to wave function treatments $[24,25]$. We note that the extensive excitation benchmark of wave function methods of Ref. 26 does unfortunately not include any member of the cyanine dye family.

The present work represents a comprehensive treatment of the simple cyanine dye series using several state-of-the-art excited-state methods such as CASPT2, QMC, CC response methods up to third approximate order, and TDDFT also in the long-range corrected and B2PLYP flavours, and the Tamm-Dancoff approximation. We give a detailed account of all parameters which may affect the calculation of the excitations in the various approaches. Our discussion focuses on the lowest bright excited state, and we enclose results for higher excited states as Supporting Information.

All computational details are given in Section 7.2. We describe the dependence of the excitation energies on the basis set and the method used to optimize the ground-state geometry in Section 7.3.1. This is followed by the excitation energies calculated with CC methods (Section 7.3.2), CASPT2 (Section 7.3.3), QMC (Section 7.3.4), and TDDFT (Section 7.3.5). In Section 7.4, we discuss the relative performance of the theoretical approaches and their comparison with experiments. Our conclusions are summarized in Section 7.5.

\subsection{Computational Details}

The ground-state structures are optimized within Hartree-Fock (HF), second-order Møller-Plesset (MP2), and density functional theory (DFT). To compute the excitation energies, we employ the complete active space self-consistent field (CASSCF) method with its perturbative extension (CASPT2), quantum Monte Carlo (QMC) methods, coupled-cluster (CC) methods, and time-dependent density functional theory (TDDFT). The CC response calculations [27, 28] are performed at the singles (CCS), singles and doubles (CCSD) [29], approximate second (CC2) [16,30-32], and approximate third (CC3) [33,34] orders. In the DFT calculations, the PBE [35], PBE0 [36-38], the CAM-B3LYP [39] and the B2PLYP [24, 25] functionals are employed. The Tamm-Dancoff-Approximation is employed in some of the TDDFT calculations and denoted with the prefix TDA [40].

The resolution-of-the-identity (RI) approximation [41] is used in all MP2 and in some CC2 calculations, and is indicated by the abbreviations RI-MP2 [42] and RICC2 [31]. All RI-MP2, RI-CC, and DFT calculations are performed with the TURBOMOLE code [43]. B2PLYP calculations are based on an unreleased TURBOMOLE implementation and the additional on top program RICC by Grimme. [24,25] The CC and CAM-B3LYP excitation energies calculated without the RI approxima- 
tion are obtained with the DALTON program suite [44]. The CAM-B3LYP excitation energy of the largest dye with the triple- $\zeta$ basis is computed with the Gaussian 09 code [45].

The complete active space calculations are performed using MOLCAS 7.2 [46]. In the CASPT2 calculations, unless otherwise stated, we employ the default IPEA zero-order Hamiltonian [23], and indicate if an additional constant level shift [47] is added to the Hamiltonian. In the CASPT2 calculations, we do not correlate as many of the lowest $\sigma$-orbitals as there are heavy atoms in the molecule. For some models, we use the Cholesky decomposition of the two-electron integrals [48] with the threshold of $10^{-8}$. The default convergence criteria are used for all calculations.

The program package CHAMP [49] is used for the QMC calculations. We employ scalar-relativistic energy-consistent Hartree-Fock pseudopotentials [50] where the carbon and nitrogen $1 s$ electrons are replaced by a non-singular $s$-non-local pseudopotential and the hydrogen potential is softened by removing the Coulomb divergence. Different Jastrow factors are used to describe the correlation with different atom types and, for each atom type, the Jastrow factor consists of an exponential of the sum of two fifth-order polynomials of the electron-nucleus and the electron-electron distances, respectively [51]. We also test the effect of including an electron-electron-nuclear term. The starting determinantal components are obtained in CASSCF calculations which are performed with the program GAMESS(US) [52], and the final CAS expansions are expressed on the CASSCF natural orbitals. The CAS wave functions of the states of interest may be truncated with an appropriate threshold on the CSF coefficients for use in the QMC calculations. The Jastrow correlation factor and the $\mathrm{CI}$ coefficients are optimized by energy minimization within VMC and, when indicated in the text, also the orbitals are optimized along with the Jastrow and CI parameters. The pseudopotentials are treated beyond the locality approximation [53] and an imaginary time step of 0.05 a.u. is used in the DMC calculations.

\subsubsection{Basis Sets and Ground-State Structures}

To investigate the basis-set dependence of the ground-state structures and of the CC and TDDFT excitations, we use the ANO-L-VXZP basis sets [54] and Dunning's correlation consistent cc-pVXZ and aug-cc-pVXZ basis sets [55-58]. For the ANO basis sets, the MOLCAS contraction scheme is employed, namely, ANOL-VDZP [3s2p1d]/[2s1p], ANO-L-VTZP [4s3p2d1f]/[3s2p1d], and ANO-L-VQZP $[5 \mathrm{~s} 4 \mathrm{p} 3 \mathrm{~d} 2 \mathrm{f}] /[4 \mathrm{~s} 3 \mathrm{p} 1 \mathrm{~d}]$. The ANO-L-VXZP basis set series is used in the CASSCF and CASPT2 calculations.

In the QMC calculations, we use the Gaussian basis sets [50] specifically constructed for our pseudopotentials. In particular, we employ the cc-pVDZ basis, denoted by $\mathrm{D}$, and the $\mathrm{T}^{\prime}$ and $\mathrm{Q}^{\prime}$ basis sets, which consist of the cc-pVDZ for hydrogen combined respectively with the cc-pVTZ and cc-pVQZ basis sets for the heavy atoms. The $\mathrm{D}+, \mathrm{T}^{\prime}+$, and $\mathrm{Q}^{\prime}+$ basis sets are constructed by augmenting the corresponding basis with diffuse $s, p$, and $d$ functions [59] on the heavy atoms. Basis 
functions with higher angular momentum than $d$ are not included in the $\mathrm{T}^{\prime}, \mathrm{T}^{\prime}+, \mathrm{Q}^{\prime}$, and $\mathrm{Q}^{\prime}+$ basis sets.

Unless indicated otherwise, the CC, CASPT2, and TDDFT excitation energies are calculated with the ANO-L-VTZP basis set, and the QMC excitations with the $\mathrm{T}^{\prime}+$ basis set. All excitation energies are computed on the RI-MP2/cc-pVQZ groundstate structures with the exception of the TDDFT excitations which are obtained using the PBE0/cc-pVQZ structures.

\subsubsection{Auxiliary Basis Sets}

In the RI-MP2/ANO-L-VXZP and RI-CC2/ANO-L-VXZP calculations, the corresponding auxiliary basis sets are not available. To assess the impact of using the ANO-L-VXZP basis sets in combination with the available aug-cc-pVXZ auxiliary basis sets, we calculate the error in the correlation energy introduced by the RI approximation for carbon and nitrogen atom, and for $\mathrm{H}_{2}$. The quantity commonly used to access the quality of an auxiliary basis set is defined as

$$
\alpha=\frac{\delta_{\mathrm{RI}}}{|\Delta \mathrm{E}(\mathrm{MP} 2)|}
$$

where $\triangle \mathrm{E}(\mathrm{MP} 2)$ is the MP2 correlation energy and $\delta_{\mathrm{RI}}$ is given by

$$
\delta_{\mathrm{RI}}=\frac{1}{4} \sum_{i<j}^{\text {occ. virt. }} \sum_{a<b} \frac{|<a b|\left|i j>_{\text {exact }}-<a b\right|\left|i j>_{\mathrm{RI}}\right|^{2}}{\epsilon_{a}-\epsilon_{i}+\epsilon_{b}-\epsilon_{j}} .
$$

The values of $\alpha$ obtained by combining the ANO-L-VXZP basis with the auxiliary aug-cc-pVXZ basis sets are given as supporting information. When the aug-ccpVQZ auxiliary basis is employed, $\alpha<0.05 \mathrm{ppm}$, which is in line with standard auxiliary-basis-optimization conditions [58]. Therefore, we adopt this auxiliary basis in all our RI calculations.

\subsubsection{Extrapolation of Excitation Energies}

The extrapolated CC3/ANO-L-VTZP (exCC3) excitation energies are obtained as

$$
\tilde{\mathrm{E}}_{\mathrm{T}}^{\mathrm{CC} 3}=\mathrm{E}_{\mathrm{T}}^{\mathrm{CC} 2}+\left(\mathrm{E}_{\mathrm{D}}^{\mathrm{CC} 3}-\mathrm{E}_{\mathrm{D}}^{\mathrm{CC} 2}\right) .
$$

This extrapolation formula is motivated by the observation that triple excitations are less basis-set sensitive than single and double excitations [60-62].

\subsection{Vertical Excitation Energies}

The cyanine dye molecules studied in this work are shown in Figure 7.1. We consider hydrogen-terminated dyes of increasing size, which we denote as CN3, CN5, $\mathrm{CN} 7, \mathrm{CN} 9$, and CN11, respectively. All hydrogen-terminated dyes have $\mathrm{C}_{2 v}$ symmetry. For these molecules, we also construct the equivalent dyes where the terminating hydrogens are substituted by methyl groups. 


\section{$\mathrm{H}_{2} \mathrm{~N} \mathrm{NH}_{2}^{+} \quad \mathrm{CN}_{3}$}

\section{$\mathrm{H}_{2} \mathrm{~N} \mathrm{NH}_{2}^{+} \quad \mathrm{CN}_{5}$}

\section{$\mathrm{H}_{2} \mathrm{~N} \mathrm{NH}_{2}^{+} \mathrm{CN}_{7}$}
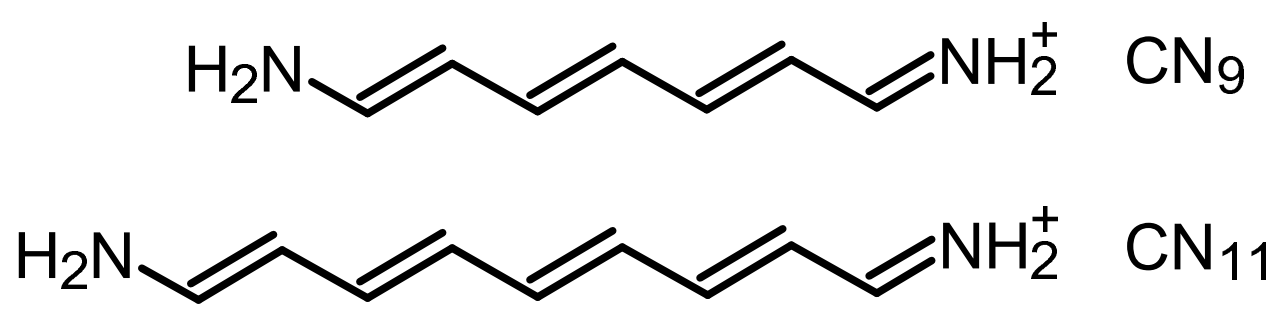

Figure 7.1: Hydrogen-terminated cyanine dyes considered in this work. Only one of the two resonant structures of each molecule is shown. The other structure can be obtained by having the first double bond at the other nitrogen atom.

\subsubsection{Basis Set Convergence and Geometry Dependence}

We employ the cc-pVXZ, aug-cc-pVXZ, and ANO-L-VXZP series to investigate the basis set dependence of the CC and CASPT2 excitations, and give a complete survey of the results in the supporting information (SI) [63]. In this section, we focus on the smallest molecule, $\mathrm{CN} 3$, since it displays the largest dependence on the basis set. The basis-set dependence of the TDDFT and QMC excitations will be discussed separately.

The CC2 excitations of CN3 as a function of the basis set are shown in Figure 7.2. The correlation-consistent basis series gives the slowest convergence in the excitation energy as a function of basis set size, and an error which is still as large as $0.15 \mathrm{eV}$ when a quadruple- $\zeta$ basis is employed. The inclusion of augmentation completely cures the problem since the energy obtained with the double- $\zeta$ basis only differs from the augmented quadruple- $\zeta$ value by $0.02 \mathrm{eV}$. The excitations computed with the ANO series converge similarly to the augmented correlation consistent values, and the use of a triple- $\zeta$ basis yields the quadruple- $\zeta$ value within better than $0.01 \mathrm{eV}$.

The behavior of the CASPT2 excitations as a function of the basis set is shown for CN3 in Figure 7.3. The excitations are obtained with the standard IPEA Hamiltonian (S-IPEA) as well as with the IPEA shift set to zero (0-IPEA) as in versions of MOLCAS prior to 6.4. The energies obtained with the IPEA Hamiltonian are $0.2 \mathrm{eV}$ higher than the values obtained without the shift and the difference is independent 


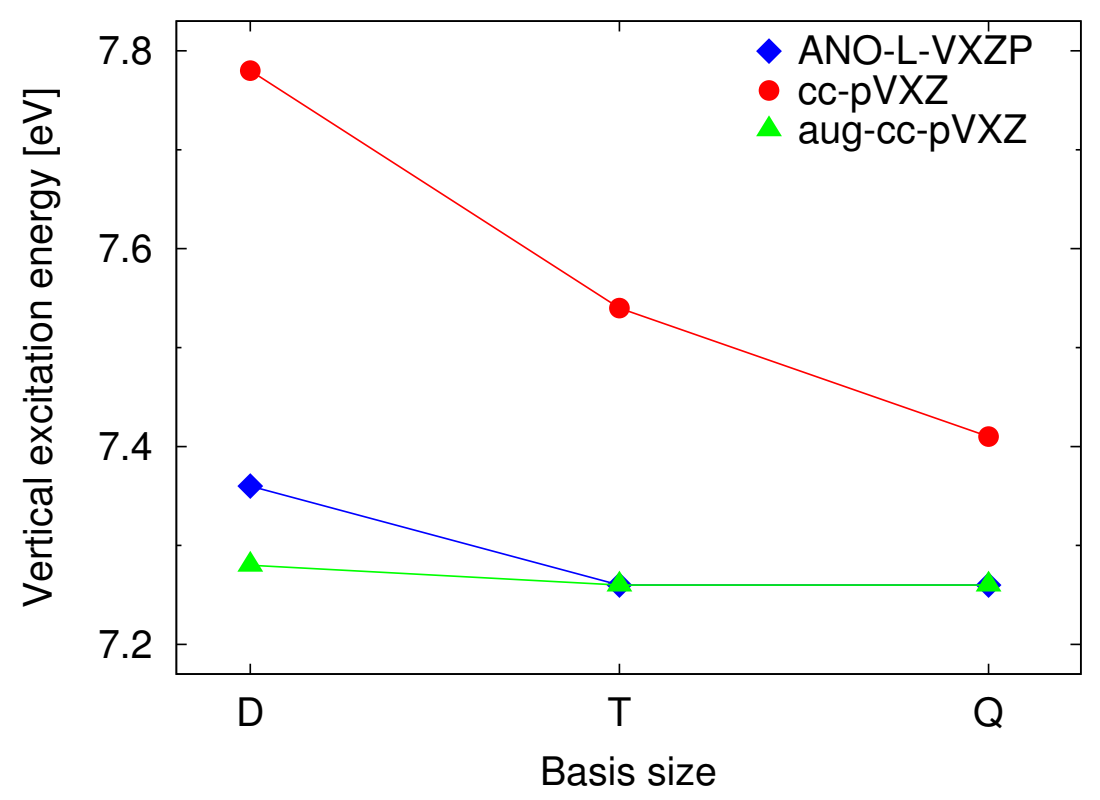

Figure 7.2: $\mathrm{CC} 2$ vertical excitation energies of $\mathrm{CN} 3$ computed with different basis sets. The ground-state MP2/cc-pVQZ geometry is used.

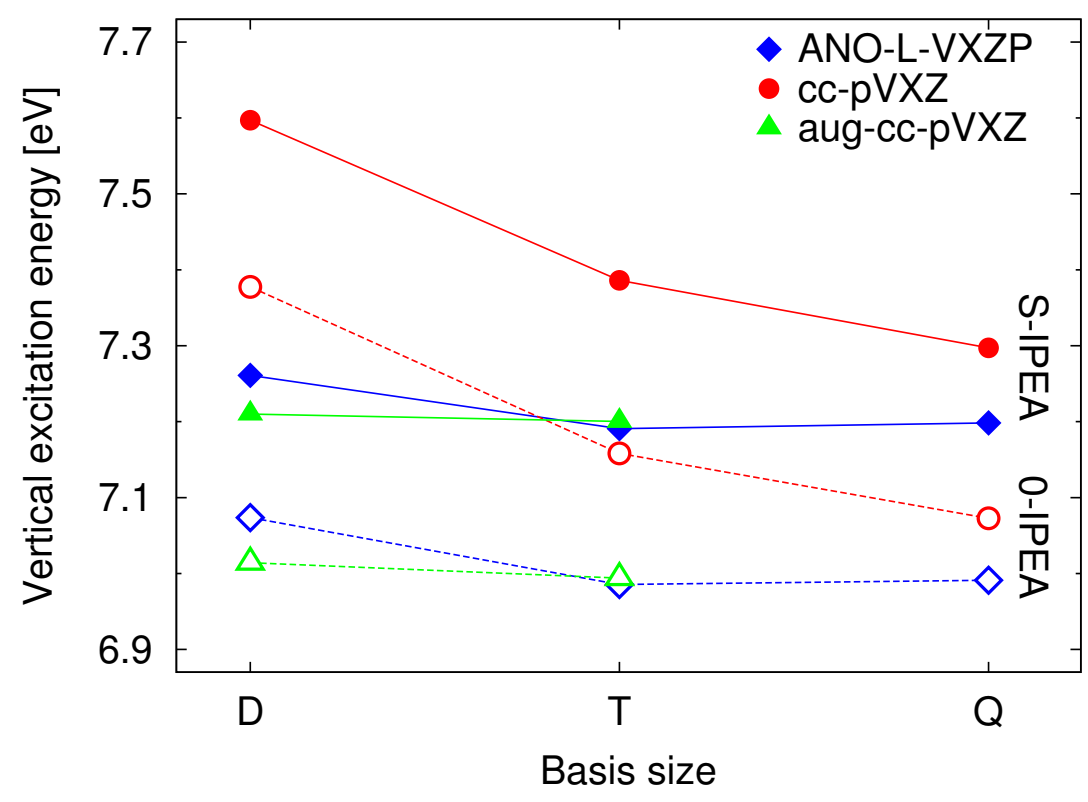

Figure 7.3: CASPT2 vertical excitation energies of CN3 computed with (S-IPEA) and without (0-IPEA) IPEA shift, and different basis sets. The ground-state MP2/ccpVQZ geometry is used. 
on the choice of the basis set. The behavior of the CASPT2 values with and without the IPEA shift closely parallels what is observed for the CC2 excitations. In particular, the inclusion of diffuse augmentation is absolutely necessary when employing the correlation consistent series while the ANO energies are well converged when a triple- $\zeta$ basis is employed.

The optimal basis set for the present system is a correlation-consistent triple- $\zeta$ basis with diffuse augmentation or an ANO triple- $\zeta$ basis. Depending on the program, segmented or generally contracted basis sets can be more efficient. As MOLCAS is optimized for generally contracted basis sets, the discussion in the following is based on ANO triple- $\zeta$ basis sets. These give CC2 and CASPT2 excitations which are well converged in the basis sets for $\mathrm{CN} 3$ as well as for the other molecules (see SI [63]). In the SI [63], we also include excitation energies calculated with the correlation consistent Dunning basis sets, more common in CC calculations. The most efficient choice in segmented contracted basis sets are the recent property-optimized basis sets by Rappoport and Furche [64]. These became available very recently, so we only include a Table with the corresponding excitations in the SI [63].

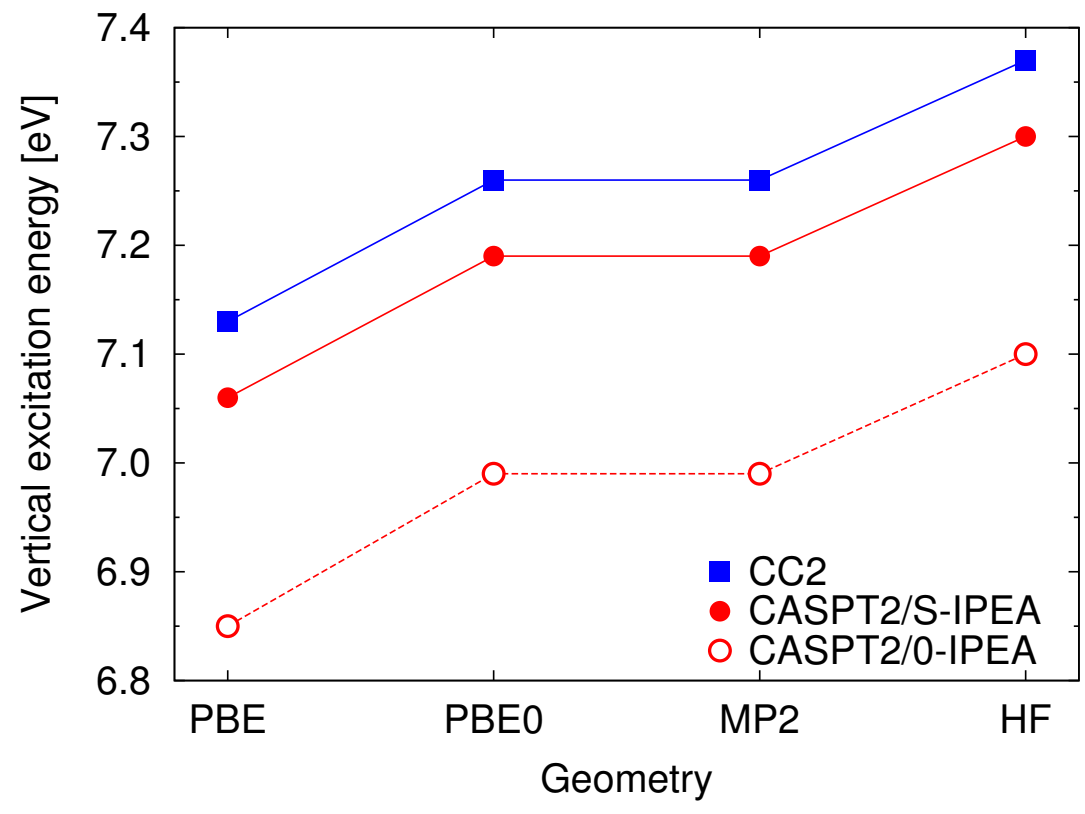

Figure 7.4: $\mathrm{CC} 2$ and CASPT2 vertical excitation energies of $\mathrm{CN} 3$ computed on different geometries. The ANO-L-VTZP basis is used.

The dependence of the CC2 and CASPT2 excitation energies on the method employed to optimize the ground-state geometry is shown for CN3 in Figure 7.4. As in the case of the basis set size, the dependence is most significant for the smallest molecule, $\mathrm{CN} 3$, as shown at the $\mathrm{CC} 2$ level in the SI [63]. Indepedently of the approach used to compute the excitations and for all chain lengths, PBE and HF geometries give the lowest and highest excitations, respectively, while PBE0 and MP2 
geometries are in between. The largest difference between the excitations computed on $\mathrm{PBE}$ and $\mathrm{HF}$ geometries is $0.24 \mathrm{eV}$ at the $\mathrm{CC} 2$ level as obtained for $\mathrm{CN} 3$, and is comparable at the CASPT2 level. The use of PBE0 and MP2 geometries gives very similar excitations with the largest difference of $0.03 \mathrm{eV}$ obtained for CN5. Throughout this work, we use MP2 or PBE0 geometries to reduce the influence of the choice of the ground-state structure on the vertical excitation, and focus on the performance of the approach employed to compute the excitations.

\subsubsection{Coupled Cluster Results}

For all dyes, we give the convergence of the $\mathrm{CC}$ excitation energies with respect to the size of the ANO basis set and the order of the CC expansion in Table 7.1. As already discussed in Section 7.3.1, the triple- $\zeta$ basis set is the most cost efficient choice as an increase to quadruple- $\zeta$ only changes the excitation energies by less than $0.01 \mathrm{eV}$. The $\mathrm{CC} 3$ calculations for the largest dye, CN11, are not feasible at the ANO-L-VTZP level, so we also compute the triple- $\zeta$ extrapolated $\mathrm{CC} 3$ results (exCC3) using Eq. 7.3. When available, the CC3 results deviate from their extrapolated counterparts by less than $0.03 \mathrm{eV}$ and the error in the extrapolation is therefore comparable to the residual basis-set error.

Table 7.1: Coupled cluster vertical excitation energies (eV) for the $1^{1} \mathrm{~B}_{1}$ state of the cyanine dye series computed at the CC2, CCSD, and CC3 level with the ANO-LVXZP basis sets. The extrapolated $\mathrm{CC} 3$ values (exCC 3 ) are obtained by adding the difference between the double- $\zeta \mathrm{CC} 3$ and $\mathrm{CC} 2$ values to the triple- $\zeta \mathrm{CC} 2$ results. The ground-state RI-MP2/cc-pVQZ structures are employed.

\begin{tabular}{lccccc}
\hline Molecule & Basis & CC2 & CCSD & CC3 & exCC3 \\
\hline CN3 & D & 7.36 & 7.32 & 7.27 & - \\
& T & 7.26 & 7.29 & 7.18 & 7.16 \\
& Q & 7.26 & 7.30 & 7.18 & - \\
CN5 & D & 5.02 & 4.98 & 4.89 & - \\
& T & 4.97 & 4.98 & 4.86 & 4.84 \\
& Q & 4.96 & 4.99 & 4.86 & - \\
CN7 & D & 3.83 & 3.79 & 3.69 & - \\
& T & 3.79 & 3.81 & 3.68 & 3.65 \\
CN9 & D & 3.13 & 3.09 & 2.99 & - \\
& T & 3.10 & 3.11 & - & 2.96 \\
CN11 & D & 2.66 & 2.62 & 2.52 & - \\
& T & $2.64^{a}$ & - & - & 2.53 \\
\hline
\end{tabular}

${ }^{a}$ Computed with the RI approximation.

The behavior of the excitation energies at different $\mathrm{CC}$ levels reflect the typical convergence of the correlation energy contribution [26]. With the ANO-L-VTZP basis, this convergence is characterized by an increase of less than $0.03 \mathrm{eV}$ when going 
from $\mathrm{CC} 2$ to the full inclusion of doubles amplitudes in CCSD, and a decrease of less than $0.14 \mathrm{eV}$ when going from $\mathrm{CC} 2$ to $\mathrm{CC} 3$. The decrease in excitation energies when going from $\mathrm{CC} 2$ to $\mathrm{CC} 3$ is larger than the one observed for the corresponding bright state in butadiene $(0.04 \mathrm{eV})$ or in the protonated Schiff base models $(0.01$ eV) $[65,66]$. The $T_{1}$-diagnostic [67] remains lower than the empirical threshold of 0.02 , indicating that the Hartree-Fock determinant is a good zeroth-order description of the ground state, and $\mathrm{CC} 2$ and CCSD results can therefore be considered reliable.

Further insight in our calculations can be gained by the amount of single- and double-excitation contribution in the CC 3 excitation energies. The single-excitation contributions decrease from $89 \%$ to $84 \%$ when going from $\mathrm{CN} 3$ to $\mathrm{CN} 11$. The double-excitation contributions increase from $11 \%$ to $16 \%$ when going from $\mathrm{CN} 3$ to CN11. This finding is in line with the growing difference between $\mathrm{CC} 2$ and $\mathrm{CC} 3$ results upon lengthening of the chain. The correlation energy strongly depends on double excitations for all molecules, and triple excitations contribute more than in the analogous polyenes and protonated Schiff bases. The ground-state correlation energy shows on the other hand little dependence on the chain length. For all molecules, $92 \%$ of the $\mathrm{CC} 3$ correlation energy is obtained already at the $\mathrm{CC} 2$ level, and the CC3 correlation energy per electron is identical up to $0.1 \mathrm{mH}$ for all dyes. This finding indicates that electron correlation effects are important mainly in the description of the excitation, for which an accurate description of correlation is therefore essential.

\subsubsection{CASPT2 Results}

The choice of the active space significantly affects the CASPT2 energies of the cyanine dyes, particularly of the smallest ones. As shown below, previous calculations [15] employed active spaces that were too small and led to underestimated CASPT2 excitation energies.

We extensively investigate the dependence of the excitations on the choice of the active space, and give a complete account of our calculations in the SI [63]. In Table 7.2, we present the most relevant subset of our results where the number of active $\pi$ orbitals of $a_{2}$ and $b_{2}$ symmetry included in the CAS is $l$ times the number of heavy atoms. This construction corresponds to $l$ atomic orbitals of $p$ character per heavy atom and produces a series of balanced active spaces. We observe that choosing $l$ equal to 2 offers a good compromise between accuracy and computational cost since the corresponding excitations are always converged to better than $0.05 \mathrm{eV}$. For the largest dye, CN11, we cannot perform a calculation with $l$ equal to 2 as the use of 22 active orbitals is not feasible. However, the excitation energy of CN11 computed with $l$ equal to 1 is converged within $0.05 \mathrm{eV}$ as can be seen from the excitations computed with larger CAS dimensions given in the SI [63].

Our optimal active space must be contrasted to the use of an active space with an equal number of active electrons and active orbitals as adopted in Ref. 15. We illustrate the shortcomings of this alternative construction by plotting the excitation of $\mathrm{CN} 3$ as a function of the dimension of the active space in Figure 7.5. The use 
Table 7.2: CASPT2 vertical excitations $(\mathrm{eV})$ of the $1^{1} \mathrm{~B}_{1}$ state computed with (SIPEA) and without (0-IPEA) IPEA shift and with different CAS $(m, n)$ expansions. All $\pi$ electrons $(m)$ in the reference are included and the number of active $\pi$ orbitals is $n=i+j$, where $i$ and $j$ are orbitals of $a_{2}$ and $b_{2}$ symmetry, respectively, as specified by the notation $[i, j]$. The number of active orbitals is a multiple $l$ of the number of heavy atoms as obtained by using $l$ atomic orbitals of $p$ character per heavy atom. We denote in boldface the optimal choice of active space in cost and accuracy for CN3-CN7. For CN9 and CN11, the maximum feasible values of 1 are 2 and 1, respectively. Additional active spaces not constructed as multiple of 1 are listed in the SI [63]. The ANO-L-VTZP basis set and the ground-state RI-MP2/ccpVQZ structures are employed.

\begin{tabular}{|c|c|c|c|c|c|}
\hline \multirow[t]{2}{*}{ Molecule } & \multicolumn{2}{|c|}{$\operatorname{CAS}(m, n)$} & \multirow[t]{2}{*}{ CASSCF } & \multicolumn{2}{|c|}{ CASPT2 } \\
\hline & $m\left[a_{2}, b_{2}\right]$ & $n\left[a_{2}, b_{2}\right]$ & & 0-IPEA & S-IPEA \\
\hline \multirow[t]{3}{*}{$\mathrm{CN} 3$} & $4[2,2]$ & $3[1,2]$ & 8.12 & 6.55 & 6.90 \\
\hline & & $6[2,4]$ & 7.56 & 6.99 & 7.19 \\
\hline & & $9[3,6]$ & 7.63 & 6.97 & 7.14 \\
\hline \multirow[t]{3}{*}{ CN5 } & $6[2,4]$ & $5[2,3]$ & 5.46 & 4.23 & 4.62 \\
\hline & & $10[4,6]$ & 5.32 & 4.46 & 4.69 \\
\hline & & $15[6,9]$ & 5.33 & 4.49 & 4.68 \\
\hline \multirow[t]{3}{*}{ CN7 } & $8[4,4]$ & $7[3,4]$ & 3.92 & 3.17 & 3.56 \\
\hline & & $14[6,8]$ & 3.91 & 3.30 & 3.52 \\
\hline & & $21[9,12]$ & 3.96 & 3.30 & 3.49 \\
\hline \multirow[t]{2}{*}{$\mathrm{CN}^{a}$} & $10[4,6]$ & $9[4,5]$ & 2.99 & 2.55 & 2.92 \\
\hline & & $18[8,10]$ & 3.13 & 2.59 & 2.81 \\
\hline $\mathrm{CN} 11^{a}$ & $12[6,6]$ & $11[5,6]$ & 2.39 & 2.10 & 2.46 \\
\hline
\end{tabular}

of a CAS $(4,4)$ space as in Ref. 15 yields an excitation which is underestimated by as much as $0.4 \mathrm{eV}$, while the excitation computed with a CAS $(4,6)$ expansion is perfectly well converged. The dependence on the size of the CAS is slightly more pronounced when the zero-order Hamiltonian with no IPEA shift is employed as in Ref. 15 and, as expected, the difference between the excitations computed with and without IPEA shift diminishes with increasing CAS size.

We summarize the CASPT2 excitations for our optimal choice of active space as a function of the ANO basis sets in Table 7.3. As discussed previously, the use of an ANO triple- $\zeta$ basis gives well converged excitations whether one uses the zero-order Hamiltonian with or without the IPEA shift. The excitations computed without the IPEA shift are $0.2 \mathrm{eV}$ lower than the values obtained with the standard IPEA Hamiltonian, independent of the basis. For CN11, the difference between 


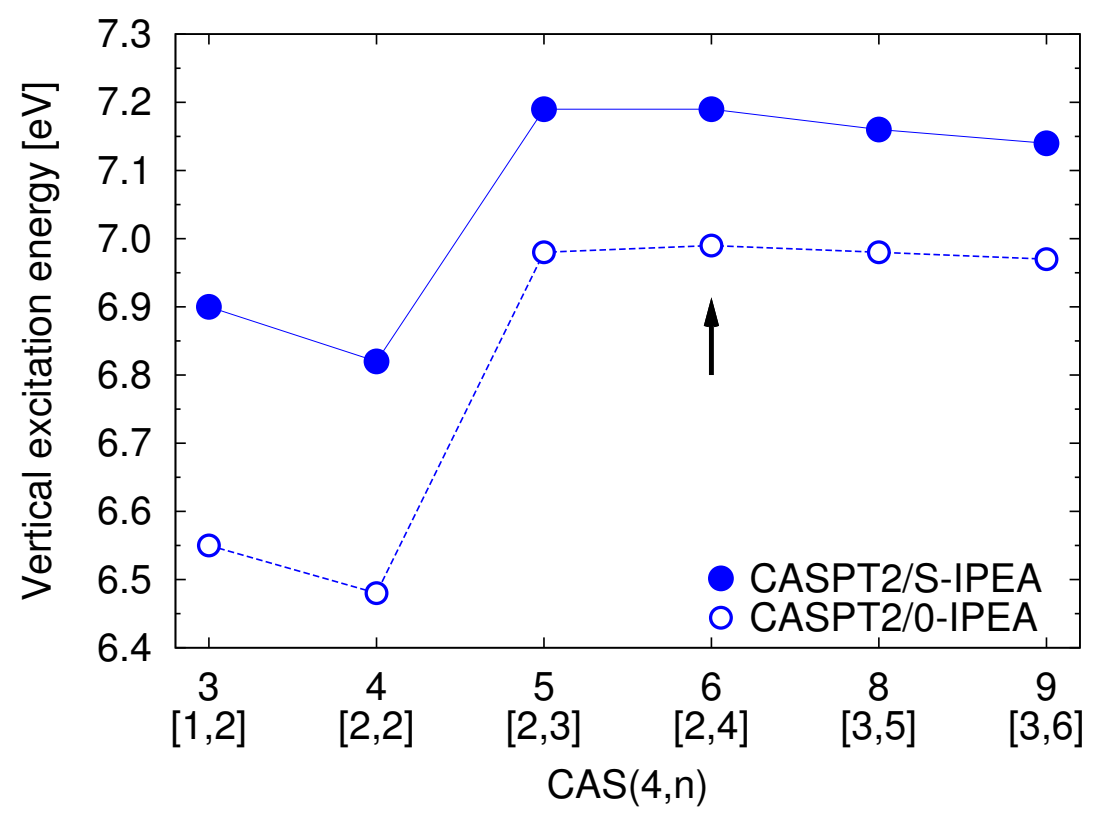

Figure 7.5: CASPT2 vertical excitation energies of $\mathrm{CN} 3$ computed with (S-IPEA) and without (0-IPEA) IPEA shift and with different $\mathrm{CAS}(4, n)$ expansions. The number of $\pi$ electrons in the reference configuration is 4 . The number of active orbitals is $n=i+j$, and $i$ and $j$ are orbitals of $a_{2}$ and $b_{2}$ symmetry, respectively, as specified by the label $[i, j]$. The arrow indicates a balanced CAS size which corresponds to use 2 atomic orbitals of $p$ character per heavy atom, and represents an optimal compromise in accuracy and cost. The CAS $(4,4)$ chosen in Ref. 15 is clearly inadequate. The ground-state MP2/cc-pVQZ geometry is used.

the values computed with and without IPEA shift appears to be larger than for the smaller dyes, and equal to $0.36 \mathrm{eV}$. The use of larger active spaces would however reduce the difference to less than $0.25 \mathrm{eV}$ also for CN11 (see SI [63]). This finding reflects the fact that CASPT2 excitations computed with the IPEA shift converge faster to the values obtained with larger CAS dimensions. 
Table 7.3: CASSCF and CASPT2 vertical excitation energies $(\mathrm{eV})$ for the $1^{1} \mathrm{~B}_{1}$ state of the cyanine dye series computed with the ANO-L-VXZP basis sets and the optimal active space. $\operatorname{A~CAS}(n, m)$ expansion is used to compute the ground- $\left(1^{1} \mathrm{~A}_{1}\right)$ and excited-state $\left(1^{1} \mathrm{~B}_{1}\right)$ energies, where $n$ and $m$ denote the number of electrons and molecular orbitals, respectively. The ground-state RI-MP2/cc-pVQZ structures are employed.

\begin{tabular}{|c|c|c|c|c|c|c|}
\hline \multirow[t]{2}{*}{ Molecule } & \multirow[t]{2}{*}{ Basis } & \multicolumn{2}{|c|}{$\operatorname{CAS}(n, m)$} & \multirow[t]{2}{*}{ CASSCF } & \multicolumn{2}{|c|}{ CASPT2 } \\
\hline & & $n\left[a_{2}, b_{2}\right]$ & $m\left[a_{2}, b_{2}\right]$ & & 0-IPEA & S-IPEA \\
\hline \multirow[t]{3}{*}{$\mathrm{CN} 3$} & $\mathrm{D}$ & $4[2,2]$ & $6[2,4]$ & 7.59 & 7.07 & 7.26 \\
\hline & $\mathrm{T}$ & & & 7.56 & 6.99 & 7.19 \\
\hline & Q & & & 7.56 & 6.99 & 7.20 \\
\hline \multirow[t]{3}{*}{ CN5 } & D & $6[2,4]$ & $10[4,6]$ & 5.25 & 4.53 & 4.74 \\
\hline & $\mathrm{T}$ & & & 5.32 & 4.46 & 4.69 \\
\hline & Q & & & 5.32 & 4.46 & 4.69 \\
\hline \multirow[t]{3}{*}{$\mathrm{CN} 7$} & $\mathrm{D}$ & $8[4,4]$ & $14[6,8]$ & 3.85 & 3.35 & 3.55 \\
\hline & $\mathrm{T}$ & & & 3.91 & 3.30 & 3.52 \\
\hline & $\mathrm{Q}^{a}$ & & & 3.92 & 3.30 & 3.53 \\
\hline \multirow[t]{3}{*}{ CN9 } & $\mathrm{D}^{a}$ & $10[4,6]$ & $18[8,10]$ & 3.08 & 2.63 & 2.83 \\
\hline & $\mathrm{T}^{a}$ & & & 3.13 & 2.59 & 2.81 \\
\hline & $\mathrm{Q}^{a}$ & & & 3.14 & 2.59 & 2.81 \\
\hline \multirow[t]{2}{*}{ CN11 } & $\mathrm{D}$ & $12[6,6]$ & $11[5,6]$ & 2.39 & 2.13 & 2.46 \\
\hline & $\mathrm{T}^{a}$ & & & 2.39 & 2.10 & 2.46 \\
\hline
\end{tabular}

${ }^{a}$ Obtained with the Cholesky decomposition with $10^{-8}$ threshold. 


\subsubsection{QMC Results}

In the determinantal component of the Jastrow-Slater wave functions, we choose the active space identified as optimal in the CASPT2 calculations, and always optimize at least the Jastrow and linear coefficients in energy minimization within variational Monte Carlo. Other ingredients in the trial wave function may impact the excitation, such as the choice of basis set, the truncation threshold on the CAS expansion, the form of the Jastrow factor, and whether one optimizes also the orbitals in the determinantal component. We investigate the effect of changing these parameters in the wave function and summarize the results in Tables 7.4 and 7.5

Most tests are performed for the smallest dye, $\mathrm{CN} 3$, whose excitation appears to be most sensitive to the features of the wave function. We find that including electron-electron-nucleus terms in the Jastrow factor has little effect on the excitation of $\mathrm{CN} 3$. While the VMC excitation slightly increases, the DMC excitation is unchanged by the presence of these additional terms in the Jastrow factor. Therefore, given the higher computational cost of these three-body terms, we only include electron-electron and electron-nucleus correlations in the Jastrow factor for all other dyes. Concerning the basis, we find that the D+ basis leads to excitations which are clearly overestimated in $\mathrm{VMC}$, while $\mathrm{T}^{\prime}+$ gives converged excitations when compared to the $\mathrm{Q}^{\prime}+$ values both in VMC and DMC. Even though the shortcomings of a $\mathrm{D}+$ basis are more visible for $\mathrm{CN} 3$ than for $\mathrm{CN} 5$, we employ a $\mathrm{T}^{\prime}+$ as default basis to compute the excitations of all dyes.

More critical for CN3 is the choice of the truncation threshold on the CAS expansion especially if one does not reoptimize the orbitals. When only the linear coefficients are reoptimized in the presence of the Jastrow factor, the DMC excitation obtained with the full CAS expansion is $0.1 \mathrm{eV}$ lower than the value computed with a threshold of 0.02 . If the orbitals are reoptimized, the DMC excitations computed with a full CAS and a truncated expansion become 0.1 and $0.2 \mathrm{eV}$ lower than the corresponding values obtained with CASSCF orbitals, and one recovers the same DMC value when employing the full or truncated CAS expansion. For CN5, the optimization of the orbitals does not significantly affect the excitations and reducing the truncation threshold on the CAS expansion has a smaller effect on the excitation than for $\mathrm{CN} 3$. Therefore, for the larger dyes, we do not reoptimize the orbitals but only make sure we have convergence with respect to the number of configuration state functions included in the determinantal component. For all dyes, we collect the best available QMC results computed with a $\mathrm{T}^{\prime}+$ and a two-body Jastrow factor in Table 7.6. 


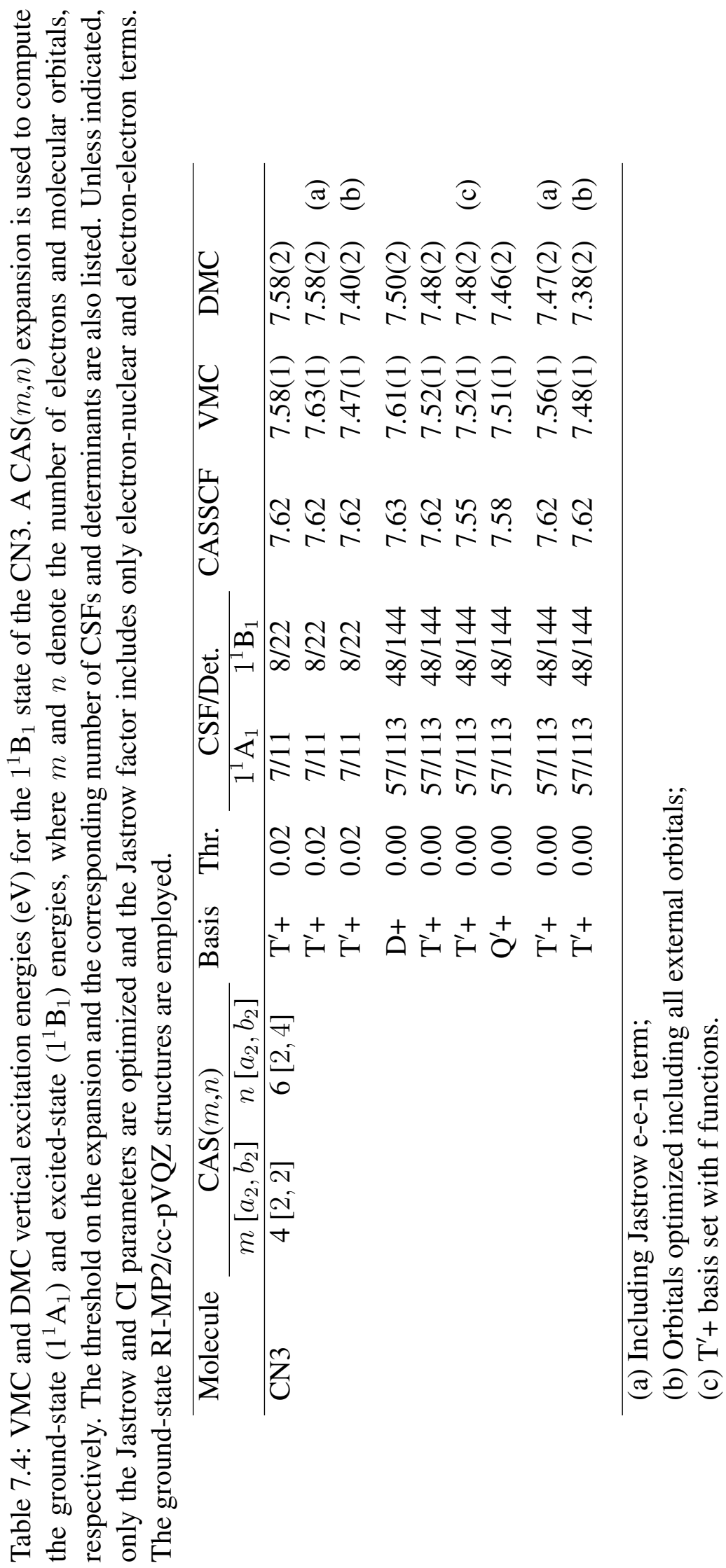




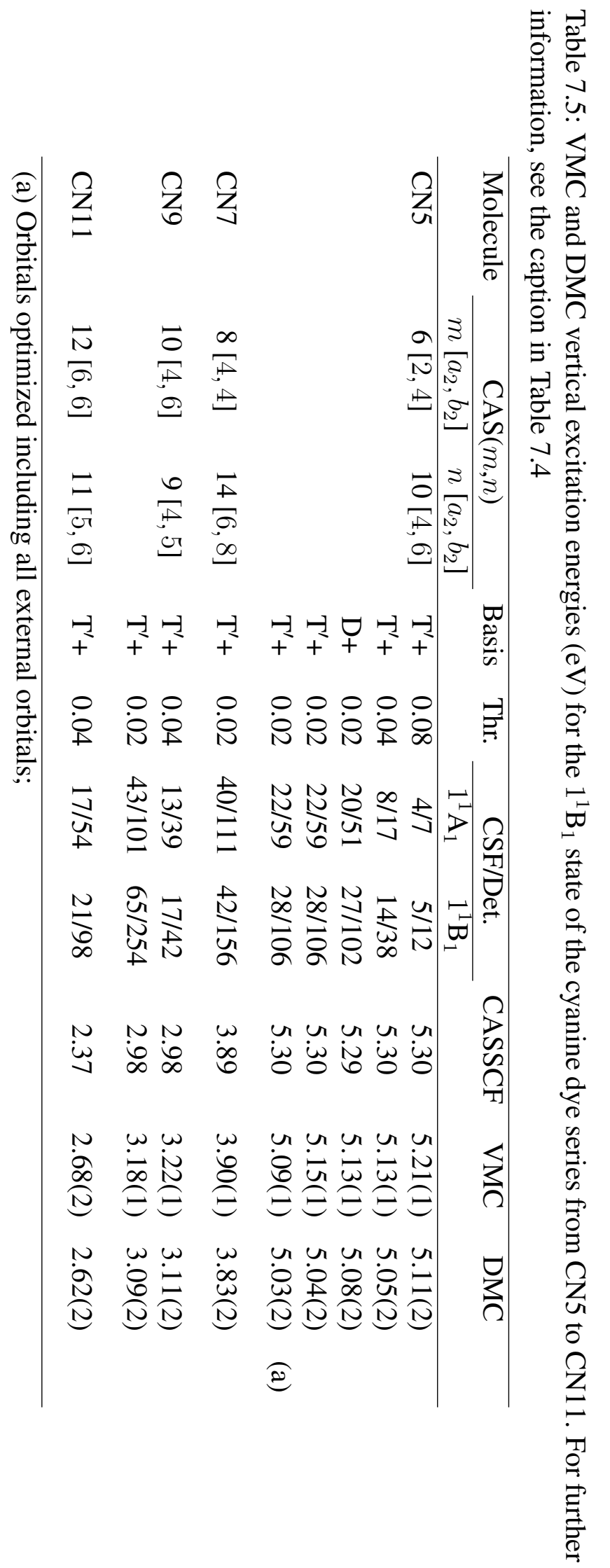


Table 7.6: VMC and DMC vertical excitation energies (eV) for the $1^{1} \mathrm{~B}_{1}$ state of the cyanine dye series. For each molecule, we show the best available value from the QMC calculations obtained using the $\mathrm{T}^{\prime}+$ basis set and a Jastrow factor including electron-nuclear and electron-electron terms. A CAS $(m, n)$ expansion is used to compute the ground-state $\left(1^{1} \mathrm{~A}_{1}\right)$ and excited-state $\left(1^{1} \mathrm{~B}_{1}\right)$ energies, where $m$ and $n$ denote the number of electrons and molecular orbitals, respectively. The threshold on the expansion is also listed. Unless indicated, only the Jastrow and CI parameters are optimized. The ground-state RI-MP2/cc-pVQZ structures are employed.

\begin{tabular}{lcccccc}
\hline Molecule & \multicolumn{2}{c}{ CAS $(m, n)$} & CASSCF & VMC & DMC & \\
\cline { 2 - 3 } & $m\left[a_{2}, b_{2}\right]$ & $n\left[a_{2}, b_{2}\right]$ & & & & \\
\hline CN3 & $4[2,2]$ & $6[2,4]$ & 7.62 & $7.48(1)$ & $7.38(2)$ & (a),(b) \\
CN5 & $6[2,4]$ & $10[4,6]$ & 5.30 & $5.09(1)$ & $5.03(2)$ & (a),(c) \\
CN7 & $8[4,4]$ & $14[6,8]$ & 3.89 & $3.90(1)$ & $3.83(2)$ & (c) \\
CN9 & $10[4,6]$ & $9[4,5]$ & 2.98 & $3.18(1)$ & $3.09(2)$ & (c) \\
CN11 & $12[6,6]$ & $11[5,6]$ & 2.37 & $2.68(2)$ & $2.62(2)$ & (d)
\end{tabular}

(a) Orbitals optimized including all external orbitals;

(b) Thr. of 0.0 ; (c) Thr. of 0.02 ; (d) Thr. of 0.04 . 


\subsubsection{TDDFT Results}

The TDDFT excitations are computed with the PBE, PBE0, and long-range corrected CAM-B3LYP functionals. We also employ the PBE0 hybrid functional in the Tamm-Dancoff approximation (TDA-PBE0) as well as the hybrid functional with a perturbative correction as proposed in Grimme's non-self-consistent B2PLYP scheme.

The TDDFT results are listed in Table 7.7, where we report the values computed with the ANO triple- $\zeta$ basis, which are converged with respect to the basis set to better than $0.02 \mathrm{eV}$ (see SI [63]). The difference between the PBE and PBE0 functionals is largest for the smallest $\mathrm{CN} 3$ dye, where the PBE excitation is 0.22 eV lower than the PBE0 result. With increasing chain length, the PBE and PBE0 excitations approach each other, only differing by $0.06 \mathrm{eV}$ for CN9. For all dyes, the CAM-B3LYP results lie between the PBE and PBE0 results with PBE giving the lowest excitation. The Tamm-Dancoff approximation and the B2PLYP scheme significantly change the excitation energies of the cyanine dyes, as already pointed out in Ref. 25. The TDA-PBE0 excitations are higher than the PBE0 results by about $0.4 \mathrm{eV}$ for $\mathrm{CN} 3$ and $0.5 \mathrm{eV}$ for the other dyes. The B2PLYP excitation energies are $0.25-0.32 \mathrm{eV}$ lower than the PBE0 results.

Table 7.7: TDDFT excitation energies $(\mathrm{eV})$ of the cyanine dye series computed with the ANO-L-VTZP basis set and different functionals. The ground-state PBE0/ccpVQZ structures are employed.

\begin{tabular}{lccccc}
\hline Molecule & PBE & PBE0 & CAM-B3LYP & B2PLYP & TDA-PBE0 \\
\hline CN3 & 7.40 & 7.62 & 7.55 & 7.30 & 8.03 \\
CN5 & 5.22 & 5.33 & 5.26 & 5.05 & 5.84 \\
CN7 & 4.11 & 4.18 & 4.12 & 3.92 & 4.71 \\
CN9 & 3.44 & 3.50 & 3.44 & 3.25 & 4.02 \\
CN11 & 2.98 & 3.03 & 2.97 & 2.80 & 3.54 \\
\hline
\end{tabular}

The excitation of the smallest dye CN3 shows the strongest dependence on the choice of the functional and, in particular, on the amount of exact exchange included in the functional. While this finding appears to support the suggestion of Ref. 25 that the self-interaction error is significant for these dyes, we note that the inclusion of exact exchange yields the same excitations as conventional generalized gradient approximations (GGA) for the larger dyes. Therefore, as we discuss in Section 7.4, the discrepancy between TDDFT and correlated methods observed also for the larger dyes cannot be simply attributed to self-interaction error.

Finally, our results follow the general trend observed for a larger set of functionals by Jacquemin et al. [68], namely, that GGA excitation energies are lower than long-range corrected hybrid-GGA values while hybrid GGAs give the largest 
excitation energies. We also note that our excitation energies deviate less than 0.08 $\mathrm{eV}$ from those of Ref. 68 and these small differences can be attributed to the use of different basis sets and ground-state structures.

\subsection{Discussion}

In this Section, we first focus on the relative performance of the theoretical approaches employed to compute the vertical excitation energies of the cyanine dyes, and then discuss their comparison with the available absorption spectra in solution.

\subsubsection{Theoretical Comparison}

Table 7.8: Vertical excitation energies $(\mathrm{eV})$ for the $1^{1} \mathrm{~B}_{1}$ state of the cyanine dye series. The CC, CASPT2/S-IPEA, and TDDFT excitations are computed with the ANO-L-VTZP basis set. The best available QMC values obtained with the $\mathrm{T}^{\prime}+$ basis set are shown.

\begin{tabular}{lccccc}
\hline Molecule & PBE0 & B2PLYP & exCC3 & CASPT2 & DMC \\
\hline CN3 & 7.62 & 7.30 & 7.16 & 7.19 & $7.38(2)$ \\
CN5 & 5.33 & 5.05 & 4.84 & 4.69 & $5.03(2)$ \\
CN7 & 4.18 & 3.92 & 3.65 & 3.52 & $3.83(2)$ \\
CN9 & 3.50 & 3.25 & 2.96 & 2.81 & $3.09(2)$ \\
CN11 & 3.03 & 2.80 & 2.53 & 2.46 & $2.62(2)$ \\
\hline
\end{tabular}

In Table 7.8, we summarize our most representative theoretical results for the vertical excitation energies of the cyanine dyes, namely, the extrapolated CC3 excitation energies (exCC3), the CASPT2 values computed with the standard IPEA Hamiltonian (CASPT2/S-IPEA), and the TDDFT energies obtained with the PBE0 functional and the B2PLYP scheme, all computed with the ANO-L-VTZP basis. We also list the best available DMC excitations computed with the $\mathrm{T}^{\prime}+$ basis set. For an extensive comparison with CC2 or CCSD, CASPT2 with no IPEA shift, and other DFT functionals and the dependence on the basis, CAS spaces, and geometries, we refer the reader to the previous sections.

Comparing wave function methods, CASPT2 gives the lowest and DMC the highest excitation energies, while exCC3 falls in between. This energetical order holds for all chain lengths except for $\mathrm{CN} 3$ where CASPT2 and exCC3 give almost identical results. The difference between CASPT 2 and exCC 3 ranges between 0.03 to $0.15 \mathrm{eV}$, and the differences are smallest for $\mathrm{CN} 3$ and $\mathrm{CN} 11$. The difference between DMC and exCC3 is of opposite sign and lies between 0.09 and $0.22 \mathrm{eV}$, and decreases steadily from $\mathrm{CN} 3$ to $\mathrm{CN} 11$.

To establish the relative accuracy of the wave function approaches, we recall that the CASPT2 method is generally quite sensitive to the choice of the zero-order 
Hamiltonian. For the cyanine dyes, the use of a Hamiltonian with no IPEA shift as it was standard prior to MOLCAS 6.4, yields excitation energies that are on average $0.2 \mathrm{eV}$ lower than the values obtained with the recommended IPEA shift of 0.25 (see Table 7.3). When the standard IPEA value is adopted, CASPT2 is in better agreement with other wave function methods indicating that this novel definition of the zero-order Hamiltonian is more accurate and represents an improvement as compared to previous CASPT2 calculations.

Previous CASPT2 calculations of the cyanine dyes by Schreiber et al. [15] are also affected by another problem, namely, an inadequate choice of the CAS space (see Figure 7.5). The combined effect of the choice of zero-order Hamiltonian and the insufficient CAS dimension explains why the CASPT2 energies of Ref. 15 are underestimated, in particular for $\mathrm{CN} 3$, where their excitation of $6.63 \mathrm{eV}$ must be compared to our value of $7.19 \mathrm{eV}$. Our excitations of the cyanine dye series should therefore be regarded as more reliable CASPT2 reference values due to the use of the IPEA Hamiltonian and a well converged size of active space.

The agreement between the exCC3 and DMC excitation energies is very satisfactory with a difference of only $0.1 \mathrm{eV}$ for the largest dyes. The larger discrepancy of $0.2 \mathrm{eV}$ for the smallest dye can be explained with the fact that the high excitation of $\mathrm{CN} 3$ is clearly more sensitive to the description of static correlation and other parameters in the wave function. For $\mathrm{CN} 3$, the DMC calculations were performed employing the full active space and optimizing also the orbital parameters. The results are stable and further improvement not obvious. When comparing with DFT methods, we will refer to the exCC3 numbers as they fall in between the CASPT2 and $\mathrm{DMC}$, keeping in mind that the exCC3 excitations, in particular for the smallest dyes, might be slightly underestimated.

The TDDFT excitations computed with the hybrid GGA PBE0 are about 0.35$0.5 \mathrm{eV}$ above the exCC3 results. As discussed in Section 7.3.5, the use of the nonhybrid GGA PBE or the long-range corrected CAM-B3LYP does not lead to a significantly closer agreement with wave function methods. The same holds for the larger number of GGA functionals including the highly parametrized Minnesota functionals tested by Jacquemin et al. [68,69]. These findings indicate a closer agreement with wave function methods can only be obtained by going beyond the GGA and hybrid-GGA levels.

It is evident that the excitation energies of the cyanine dyes are sensitive to the correlation energy treatment. This can be seen in the spread observed among the wave function methods and, at the TDDFT level, from the TDA-PBE0 results. Application of the TDA further deteriorates the agreement with the wave function methods (see Table 7.7). Within the Tamm-Dancoff approximation, matrix elements that mix excitations and deexcitations are neglected so that the excited state is described by excitations only. It has already been stressed by Grimme and Neese [25] that the present cyanines are one of the rare cases where deexcitations substantially contribute to the excitation energy. Clearly, with the omission of the deexcitations, an important component of correlation energy is neglected.

The only TDDFT approach that significantly improves the agreement with the 
wave function methods is B2PLYP. The deviation from the exCC3 results ranges between $0.14-0.29 \mathrm{eV}$ and increases from $\mathrm{CN} 3$ to $\mathrm{CN} 11$. The agreement between B2PLYP and DMC is almost perfect for the smaller dyes and the difference increases to 0.2 for the larger models. Therefore, the discrepancy with either exCC3 or DMC increases for excitations that have a larger double excitation character (as seen in the exCC3 calculations). The improvement given by the use of the B2PLYP scheme comes however at the cost of an increase in computational scaling, the introduction of an additional empirical parameter, and other well-known limitations [25].

The improved behavior of B2PLYP with respect to GGA or hybrid functionals can be understood from the presence of the additional perturbative correction. The non-self-consistent B2PLYP correction is analogous to the (D)-correction in CIS(D) excitation energies, or the MP2 energy correction in the ground state, but computed with Kohn-Sham and not Hartree-Fock orbitals. B2PLYP is therefore an empirical perturbative way to incorporate some double-excitation character into the TDDFT excitation energies. In the ground state, the opposite-spin part of the MP2 energy correction is identical to the first non-vanishing order of the RPA correlation energy as shown by Eshuis et al. [70]. In the excited state, the good performance of B2PLYP is thus an indication that the use of exact RPA correlation may cure the shortcomings of TDDFT in the cyanine dyes by a satisfactory description of double-excitation character. A non-empirical route to incorporate double excitations into TDDFT has been formulated and applied for instance by Cave et al. on polyenes [71].

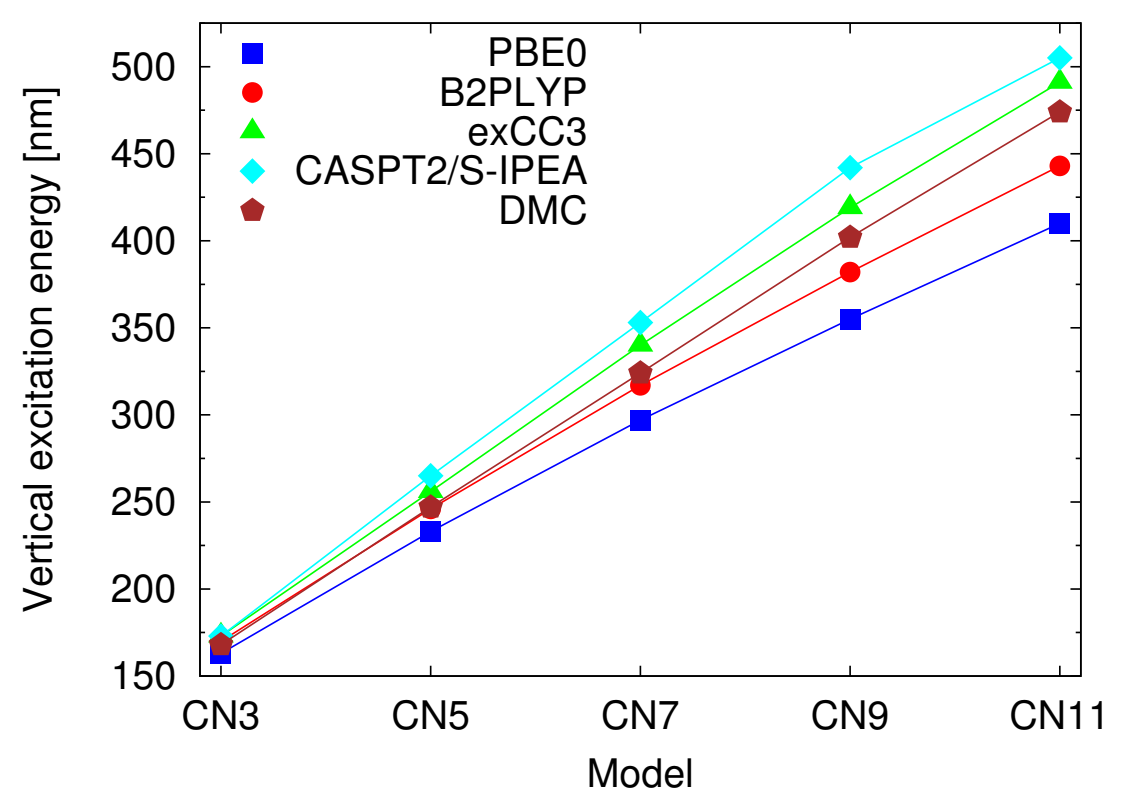

Figure 7.6: Vertical excitation energies of the cyanine dye series in $\mathrm{nm}$. The exCC3, CASPT2/S-IPEA, and TDDFT results are computed with the ANO-L-VTZP basis set. The best available DMC values obtained with the $\mathrm{T}^{\prime}+$ basis set are shown. 
Finally, as it is customary when discussing the cyanine dye series, we show the theoretical excitation energies in $\mathrm{nm}$ in Figure 7.6. All methods appear to follow the almost linear behaviour traditionally called the vinyl shift.

\subsubsection{Comparison with Experiments}

Table 7.9: Experimental absorption maximum (eV) of the cyanine dye series for different solutions and substitutions at the nitrogen atoms $\left(\mathrm{R}_{1}, \mathrm{R}_{2}\right)$. The dielectric constant and the corresponding experimental temperature are given in brackets.

\begin{tabular}{lccc}
\hline & \multicolumn{3}{c}{ Nitrogen termination $\left(\mathrm{R}_{1}, \mathrm{R}_{2}\right)$} \\
\cline { 2 - 4 } Molecule & $(\mathrm{H}, \mathrm{H})$ & $(\mathrm{H}, \mathrm{Me})$ & $(\mathrm{Me}, \mathrm{Me})$ \\
\hline $\mathrm{CN} 3$ & - & - & $5.54^{d}$ \\
$\mathrm{CN} 5$ & $4.34^{b}$ & $4.20^{b}, 4.19^{c}$ & $3.97^{a}, 4.01^{c}, 3.96^{d}$ \\
$\mathrm{CN} 7$ & $3.28^{b}$ & $3.15^{b}, 3.14^{c}$ & $3.01^{e}, 2.99^{a}, 3.02^{c}, 2.98^{d}$ \\
$\mathrm{CN} 9$ & - & $2.53^{b}, 2.51^{c}$ & $2.40^{a}, 2.44^{c}, 2.39^{d}$ \\
$\mathrm{CN} 11$ & - & - & $1.96^{a}, 2.03^{c}, 1.98^{d}$ \\
\hline${ }^{a}$ Ref. 72, measured in Methylendichloride $\left(9.1,20.0^{\circ} \mathrm{C}\right)$. \\
${ }^{b}$ Ref. 73, measured in $\mathrm{H}_{2} \mathrm{O}\left(80.4,20.0^{\circ} \mathrm{C}\right)$. \\
${ }^{c}$ Ref. 74, measured in Methanol $\left(32.6,25^{\circ} \mathrm{C}\right)$. \\
${ }^{d}$ Ref. 75, measured in Methylendichloride $\left(9.1,20.0^{\circ} \mathrm{C}\right)$. \\
${ }^{e}$ Ref. 76, measured in Ethanol $\left(24.3,25^{\circ} \mathrm{C}\right)$.
\end{tabular}

In Table 7.9, we collect the experimental absorption maxima for comparison with the computed vertical excitation energies. The experimental spectra were recorded in different solvents in the presence of $\mathrm{ClO}_{4}^{-}$counterions, and all show broad absorption maxima for the lowest excited state. The position of the absorption maxima depends only negligibly on the dielectric constant of the solvent with variations smaller than $0.07 \mathrm{eV}$. Most experimental values were recorded for cyanine dyes with two methyl-substituents on each nitrogen, and the absorption maxima of the methylated species are shifted to lower energies compared to the values of the unmethylated counterparts. The experimental methyl shift is $0.33-0.38 \mathrm{eV}$ for CN5 and 0.26-0.30 $\mathrm{eV}$ for CN7 depending on the solvent.

As shown in Table 7.10, the experimental shifts upon methylation are theoretically well reproduced at the $\mathrm{CC} 2$ level with a value of $0.39 \mathrm{eV}$ and $0.26 \mathrm{eV}$ for $\mathrm{CN} 5$ and CN7, respectively, but largely overestimated by TDDFT/PBE0. The methyl shift in the CC2 excitations diminishes from $1.19 \mathrm{eV}$ for $\mathrm{CN} 3$ to $0.17 \mathrm{eV}$ for $\mathrm{CN} 11$ as expected since the influence of the end groups should vanish in large molecular chains. The geometries of the methylated dyes are obtained in $\mathrm{C}_{2 v}$ symmetry but relaxing the symmetry constraint does not change the structure of the dyes with the exception of $\mathrm{CN} 3$, where steric interaction between methyl groups at different nitrogens forces the $\mathrm{CN} 3$ dye into a non-planar structure of $\mathrm{C}_{2}$ symmetry. Recomputing 
Table 7.10: RI-CC2 and TDDFT/PBE0 excitation energy (eV) of the $1^{1} \mathrm{~B}_{1}$ state for the methylated streptocyanine dye series computed with the ANO-L-VTZP basis sets. The RI-MP2/cc-pVQZ and PBE0/cc-pVQZ ground-state structures in $\mathrm{C}_{2 v}$ symmetry are employed for the CC2 and TDDFT calculations, respectively.

\begin{tabular}{lccccc}
\hline Molecule & \multicolumn{2}{c}{$\mathrm{CC} 2$} & & \multicolumn{2}{c}{ TDDFT/PBE0 } \\
\cline { 2 - 3 } \cline { 5 - 6 } & $(\mathrm{H}, \mathrm{H})$ & $(\mathrm{Me}, \mathrm{Me})$ & & $(\mathrm{H}, \mathrm{H})$ & $(\mathrm{Me}, \mathrm{Me})$ \\
\hline CN3 & 7.26 & 6.07 & & 7.62 & 6.00 \\
CN5 & 4.97 & 4.58 & & 5.33 & 4.75 \\
CN7 & 3.79 & 3.53 & & 4.18 & 3.81 \\
CN9 & 3.10 & 2.90 & & 3.50 & 3.23 \\
CN11 & 2.64 & 2.47 & & 3.03 & 2.82 \\
\hline
\end{tabular}

the excitation energies at the CASPT2 and CC2 level on the $\mathrm{C}_{2}$ structure of $\mathrm{CN} 3$ only increases the excitations by $0.07 \mathrm{eV}$ and $0.06 \mathrm{eV}$, respectively.

The basis for a comparison between computed vertical excitation energies and experimental absorption maxima is the assumption that the transition probability is largest at the ground-state minimum and when the transition is vertical, that is, when ground- and excited-state structures are identical. Examples where these assumptions are not satisfied are numerous $[77,78]$ but we restrict the discussion here to the validity of the comparison for the cyanine dye series.

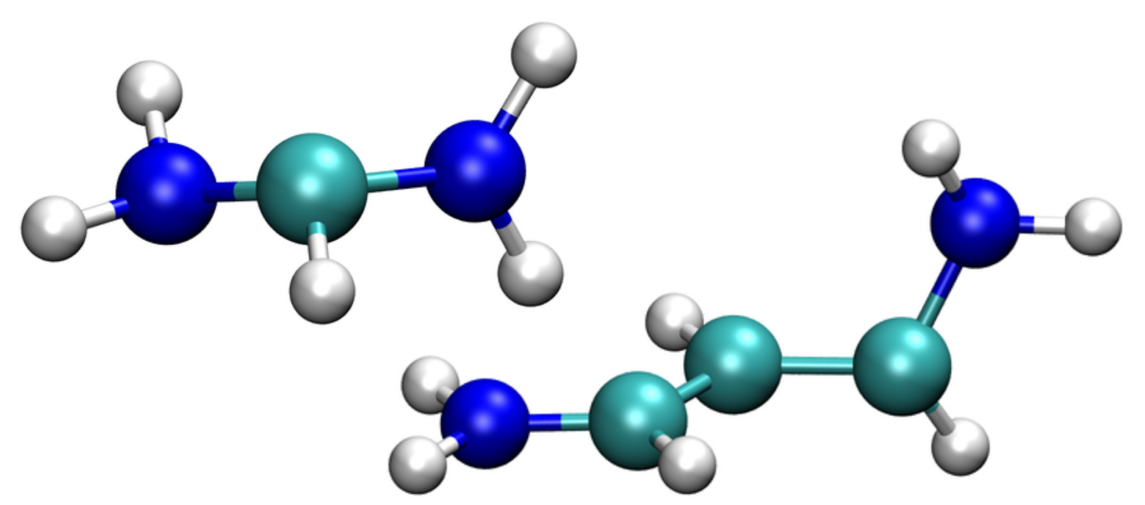

Figure 7.7: Excited-state minimal geometries of the $\mathrm{CN} 3$ and $\mathrm{CN} 5$ dyes obtained with RI-CC2 and the ANO-L-VTZP basis sets.

The calculation of the absorption spectrum of the cyanine dyes using standard schemes is not possible here since it requires the existence of an excited-state minimum. Relaxing the excited state in $\mathrm{C}_{2 v}$ symmetry at the CC2 level yields Stokes shifts of almost $1 \mathrm{eV}$ as shown in Table 7.11. Further relaxation of CN3 and CN5 without symmetry constraints leads to the highly twisted structures shown in Figure 7.7. Clearly, absorption spectra based on harmonic potentials in the excited state cannot be calculated for these systems. 
Table 7.11: Vertical and constrained-adiabatic excitation energies $(\mathrm{eV})$ for the $1^{1} \mathrm{~B}_{1}$ state, obtained with RI-CC2 and the ANO-L-VTZP basis sets. Excited-state geometry optimizations are restricted to $\mathrm{C}_{2 v}$ symmetry. The stokes shift is the difference between vertical and $\mathrm{C}_{2 v}$-constrained adiabatic excitation energy. Relaxing the planarity constraint for $\mathrm{CN} 3$ and $\mathrm{CN} 5$ indicates that there is no planar minimum.

\begin{tabular}{lccc}
\hline Molecule & \multicolumn{2}{c}{$\mathrm{E}_{\mathrm{exc}}(\mathrm{eV})$} & Stokes shift $\mathrm{C}_{2 \mathrm{v}}$ \\
\cline { 2 - 3 } & Vertical & Adiabatic $\mathrm{C}_{2 v}$ & \\
\hline CN3 & 7.26 & 6.29 & 0.97 \\
CN5 & 4.97 & 4.64 & 0.33 \\
CN7 & 3.79 & 3.65 & 0.14 \\
CN9 & 3.10 & 3.01 & 0.09 \\
CN11 & 2.64 & 2.58 & 0.06 \\
\hline
\end{tabular}

The large Stokes shifts given in Table 7.11 explain the broad absorption maxima in the experiments. Within slight variation of the ground-state geometry, a large number of vibrational states at different energies can be reached if the FranckCondon region of the excited state is distant from any minimum. Moreover, the fact that a relaxed long-lived excited-state structure is most likely non-existent also increases the likelihood of non-vertical transitions. We therefore conclude that the comparison between the computed vertical excitation energies and the experimental absorption maxima is not reliable, and certainly not suitable to assess the performance of high-level computational methods.

In fact, the direct comparison of calculated vertical excitation energies and experimental absorption maxima shows that $\mathrm{CC} 2$ results for the methylated dyes are on average $0.5 \mathrm{eV}$ above the experimental data. The differences range from 0.44 $\mathrm{eV}$ for $\mathrm{CN} 11$ to $0.62 \mathrm{eV}$ for $\mathrm{CN} 5$ and are dependent on the solvent. The deviations of the vertical excitations from the absorption maxima for the methylated species are consistent with the values obtained for the unmethylated dyes. Our CASPT2 excitation energies computed with the recommended IPEA zero-order Hamiltonian and carefully converged dimensions of the active space lie $0.34-0.35 \mathrm{eV}$ above the experimental values. The apparently better agreement obtained in the older work by Schreiber et al. can be explained with their use of an inadequate active space as well as the use of a different zero-order Hamiltonian. The zero-order Hamiltonian used in our work was introduced a few years after the publication of Schreiber's results, and is on average more accurate.

In summary, all methods give vertical excitation energies above the experimental absorption maxima with CASPT2 yielding the lowest values but still more than 0.3 $\mathrm{eV}$ higher than the experiments. The different wavefunction approaches yield very similar results, and all lie well above the experimental values. This supports our notion that the experimental absorption maxima correspond to nonvertical transitions. The influence of the solvent and the counterion are not included in our computational description and may further contribute to the discrepancy between theory and experiment. 


\subsection{Conclusion}

For almost a decade, the simple cyanine dyes studied in this work have represented an intriguing and problematic case for TDDFT and a challenge for the development of new density functionals. The availability of accurate theoretical vertical excitations for these dyes is therefore very important to assess the performance of existing or novel TDDFT approaches. With the present work, we offer carefully benchmarked reference values computed with CASPT2, QMC, CC, and various flavours of TDDFT as aid for future developments. Our analysis based on such a large variety of excited-state methods gives a broad perspective on the parameters influencing the excited-state description.

We find that previous CASPT2 calculations [15] do not offer a reliable benchmark for the cyanine dyes since the chosen active space was inadequate and led to a severe underestimation of the CASPT2 excitations, with errors as large as 0.6 $\mathrm{eV}$ for the smallest CN3 dye. Our CASPT2 calculations are superior to these older studies in the use of the improved zero-order IPEA Hamiltonian and a balanced and well converged choice of the active space. Even though the empiricism introduced by the choice of zeroth-order Hamiltonian renders the assessment of CASPT2 calculations more difficult, the CASPT2 excitations obtained with the recommended IPEA shift appear to be more reliable than those computed without this shift, as the IPEA values are energetically closer to the extrapolated CC3 and DMC results. With our improved CASPT2 vertical excitations, we find that the agreement among all wavefunction methods is generally quite reasonable with the largest deviations being observed for the smaller dyes, which appear most sensitive to the treatment of static correlation.

Consequently, the overestimation attributed in past to TDDFT when comparing to older CASPT2 calculations is now not as severe. Nevertheless, the performance of standard GGA and hybrid GGA functionals is not satisfactory and our calculations indicate that the discrepancy between TDDFT and wave function methods is due to an insufficient description of double-excitation character at the GGA level. The B2PLYP functional is an empirical scheme to partially incorporate double excitation character and it significantly improves the description in the cyanine dyes, showing the best agreement with $\mathrm{CC} 3$ and DMC results.

Since all wavefunction methods are in close agreement and the calculations appear rather robust, we consider the corresponding excitations trustworthy. It therefore remains an open question why the theoretical results disagree with the location of the absorption maxima in the experimental spectra in solution. Quite surprisingly, we find that the addition of methylation significantly lowers the vertical excitations of the smallest dyes bringing them in closer agreement with the experimental absorption maxima of the methylated species. Nevertheless, the remaining discrepancy between theory and experiment is quite large and we attribute it to the presence of non-vertical transitions. In principle, one could prove or disprove this statement by a direct simulation of the absorption spectra. However, these simulations are not straightforward due to the lack of excited-state harmonic potentials and excited-state 
minima. We find that the relaxation of some of the smaller dyes in planar symmetry leads to Stokes shifts as large as $1 \mathrm{eV}$ and further unconstrained relaxation yields highly distorted structures that render the reconstruction of the spectra impossible. Clearly, a direct comparison of the experimental absorption maxima and the vertical excitation energies is not reliable and should not constitute the basis for the assessment of theoretical methods.

\subsection{Bibliography}

[1] in Light Absorption of Organic Colorants, Vol. 12 of Reactivity and Structure Concepts in Organic Chemistry, edited by J. Fabian and H. Hartmann (Springer, Heidelberg, 1980), p. 162.

[2] D. Strack, T. Vogt, and W. Schliemann, Phytochemistry 62, 247 (2003).

[3] A. Mishra, M. K. R. Fischer, and P. Bäuerle, Angew. Chem. Int. Ed. 48, 2474 (2009).

[4] H. Mustroph, M. Stollenwerk, and V. Bressau, Angew. Chem. Int. Ed. 45, 2016 (2006).

[5] R. Weissleder and V. Ntziachristos, Nature Medicine 9, 123 (2003).

[6] F. Würthner, J. Schmidt, M. Stolte, and R. Wortmann, Angew. Chem. 118, 3926 (2006).

[7] S. Ikeda, T. Kubota, M. Yuki, and A. Okamoto, Angew. Chem. Int. Ed. 48, 6480 (2009).

[8] N. S. Bayliss, Q. Rev. Chem. Soc. 6, 319 (1952).

[9] I. Paci, J. C. Johnson, X. Chen, G. Rana, D. Popovic, D. E. David, A. J. Nozik, M. A. Ratner, and J. Michl, J. Am. Chem. Soc. 128, 16546 (2006).

[10] M. E. Casida, in Recent Advances in Density Functional Methods, Part I, edited by D. P. Chong (PUBLISHER, 1995), p. 155.

[11] F. Furche, J. Chem. Phys. 114, 5982 (2001).

[12] D. Rappoport and F. Furche, in Time-Dependent Density Functional Theory, No. 706 in Lecture Notes in Physics, edited by M. A. L. Marques, C. A. Ullrich, F. Nogueira, A. Rubio, K. Burke, and E. K. Gross (PUBLISHER, 2006), pp. 337-354.

[13] D. Rappoport and F. Furche, Phys. Chem. Chem. Phys. 11, 6353 (2009).

[14] J. Fabian, Dyes and Pigments 84, 36 (2010). 
[15] M. Schreiber, V. Buß, and M. P. Fülscher, Phys. Chem. Chem. Phys. 3, 3906 (2001).

[16] C. Hättig and A. Köhn, J. Chem. Phys. 117, 6939 (2002).

[17] D. Rappoport and F. Furche, J. Chem. Phys. 126, 201104 (2007).

[18] R. Send and F. Furche, J. Chem. Phys. 131, 044107 (2010).

[19] F. Cordova, L. J. Doriol, A. Ipatov, M. E. Casida, C. Filippi, and A. Vela, J. Chem. Phys. 127, 164111 (2007).

[20] E. Tapavicza, I. Tavernelli, U. Rothlisberger, C. Filippi, and M. E. Casida, J. Chem. Phys. 129, 124108 (2008).

[21] C. Filippi, M. Zaccheddu, and F. Buda, J. Chem. Theory Comput. 5, 2074 (2009).

[22] O. Valsson and C. Filippi, 6, 1275 (2010).

[23] G. Ghigo, B. O. Roos, and P.-Å. Malmqvist, Chem. Phys. Lett. 396, 142 (2004).

[24] S. Grimme, J. Chem. Phys. 124, 034108 (2006).

[25] S. Grimme and F. Neese, J. Chem. Phys. 127, 154116 (2007).

[26] M. Schreiber, M. R. Silva-Junior, S. P. A. Sauer, and W. Thiel, J. Chem. Phys. 128, 134110 (2008).

[27] J. Olsen and P. Jørgensen, J. Chem. Phys. 82, 3235 (1985).

[28] H. Koch and P. Jørgensen, J. Chem. Phys. 93, 3333 (1990).

[29] G. D. Purvis and R. J. Bartlett, J. Chem. Phys. 76, 1910 (1982).

[30] O. Christiansen, H. Koch, and P. Jørgensen, Chem. Phys. Lett. 243, 409 (1995).

[31] C. Hättig and F. Weigend, J. Chem. Phys. 113, 5154 (2000).

[32] C. Hättig, Adv. in Quantum. Chem. 50, 37 (2005).

[33] O. Christiansen, H. Koch, and P. Jørgensen, J. Chem. Phys. 103, 7429 (1995).

[34] H. Koch, O. Christiansen, P. Jørgensen, A. M. S. de Merás, and T. Helgaker, J. Chem. Phys. 106, 1808 (1997).

[35] J. P. Perdew, K. Burke, and M. Ernzerhof, Phys. Rev. Letters 77, 3865 (1996).

[36] J. P. Perdew, K. Burke, and M. Ernzerhof, J. Chem. Phys. 105, 9982 (1996). 
[37] C. Adamo and V. Barone, J. Chem. Phys. 110, 6158 (1999).

[38] M. Ernzerhof and G. E. Scuseria, J. Chem. Phys. 110, 5029 (1999).

[39] T. Yanai, D. P. Tew, and N. C. Handy, Chem. Phys. Lett. 393, 51 (2004).

[40] S. Hirata and M. Head-Gordon, Chem. Phys. Lett. 314, 291 (1999).

[41] K. Eichkorn, O. Treutler, H. Öhm, M. Häser, and R. Ahlrichs, Chem. Phys. Lett. 240, 283 (1995).

[42] F. Weigend, M. Häser, H. Patzelt, and R. Ahlrichs, Chem. Phys. Letters 294, 143 (1998).

[43] TURBOMOLE 6.2, TURBOMOLE GmbH, Karlsruhe, http://www.turbomole.com (2010).

[44] DALTON, an ab initio electronic structure program, release 2.0, see http://www.kjemi.uio.no/software/dalton/dalton.html (2005).

[45] M. J. Frisch, G. W. Trucks, H. B. Schlegel, G. E. Scuseria, M. A. Robb, J. R. Cheeseman, G. Scalmani, V. Barone, B. Mennucci, G. A. Petersson, H. Nakatsuji, M. Caricato, X. Li, H. P. Hratchian, A. F. Izmaylov, J. Bloino, G. Zheng, J. L. Sonnenberg, M. Hada, M. Ehara, K. Toyota, R. Fukuda, J. Hasegawa, M. Ishida, T. Nakajima, Y. Honda, O. Kitao, H. Nakai, T. Vreven, J. A. Montgomery, Jr., J. E. Peralta, F. Ogliaro, M. Bearpark, J. J. Heyd, E. Brothers, K. N. Kudin, V. N. Staroverov, R. Kobayashi, J. Normand, K. Raghavachari, A. Rendell, J. C. Burant, S. S. Iyengar, J. Tomasi, M. Cossi, N. Rega, J. M. Millam, M. Klene, J. E. Knox, J. B. Cross, V. Bakken, C. Adamo, J. Jaramillo, R. Gomperts, R. E. Stratmann, O. Yazyev, A. J. Austin, R. Cammi, C. Pomelli, J. W. Ochterski, R. L. Martin, K. Morokuma, V. G. Zakrzewski, G. A. Voth, P. Salvador, J. J. Dannenberg, S. Dapprich, A. D. Daniels, Ö. Farkas, J. B. Foresman, J. V. Ortiz, J. Cioslowski, and D. J. Fox, Gaussian 09 Revision A.02, Gaussian Inc. Wallingford CT 2009.

[46] G. Karlström, R. Lindh, P.-Å. Malmqvist, B. O. Roos, U. Ryde, V. Veryazov, P.-O. Widmark, M. Cossi, B. Schimmelpfennig, P. Neogrady, and L. Seijo, Comput. Mater. Sci. 28, 222 (2003).

[47] B. O. Roos and K. Andersson, Chem. Phys. Lett. 245, 215 (1995).

[48] F. Aquilante, P.-Å. Malmqvist, T. B. Pedersen, A. Ghosh, and B. O. Roos, J. Chem. Theory Comput. 4, 694 (2008).

[49] CHAMP is a quantum Monte Carlo program package written by C. J. Umrigar, C. Filippi and collaborators.

[50] M. Burkatzki, C. Filippi, and M. Dolg, J. Chem. Phys. 126, 234105 (2007). 
[51] C. Filippi and C. J. Umrigar, J. Chem. Phys. 105, 213 (1996), as Jastrow correlation factor, we use the exponential of the sum of three fifth-order polynomials of the electron-nuclear (e-n), the electron-electron (e-e), and of pure 3-body mixed e-e and e-n distances, respectively. The Jastrow factor is adapted to deal with pseudo-atoms, and the scaling factor $\kappa$ is set to 0.6 a.u.

[52] M. W. Schmidt, K. K. Baldridge, J. A. Boatz, S. T. Elbert, M. S. Gordon, J. H. Jensen, S. Koseki, N. Matsunaga, K. A. Nguyen, S. Su, T. L. Windus, M. Dupuis, and J. A. M. Jr, J. Comput. Chem. 14, 1347 (1993).

[53] M. Casula, Phys. Rev. B 74, 161102 (2006).

[54] P. Widmark, P. Malmqvist, and B. O. Roos, Theor. Chem. Acc. 77, 291 (1990).

[55] T. H. Dunning Jr, J. Chem. Phys. 90, 1007 (1989).

[56] K. A. Peterson, D. E. Woon, and T. H. Dunning Jr, J. Chem. Phys. 100, 7410 (1994).

[57] A. Wilson, T. van Mourik, and T. H. Dunning Jr, J. Mol. Struct. (Theochem) 388, 339 (1997).

[58] F. Weigend, Phys. Chem. Chem. Phys. 8, 1057 (2006).

[59] We take the diffuse functions for the carbon and nitrogen atoms from the augcc-pVXZ basis sets in the EMSL Basis Set Library (http://bse.pnl.gov). In the $\mathrm{D}+$ basis, we add the diffuse $s$ and $p$ functions from the aug-cc-pVDZ basis. In the $\mathrm{T}^{\prime}+$ and $\mathrm{Q}^{\prime}+$ basis sets, we add the diffuse $s, p$, and $d$ functions from the aug-cc-pVTZ and aug-cc-pVQZ basis, respectively.

[60] W. Klopper, J. Noga, H. Koch, and T. Helgaker, Theor. Chem. Acc. 97, 164 (1997).

[61] A. Karton, P. R. Taylor, and J. M. L. Marian, J. Chem. Phys. 127, 064104 (2007).

[62] M. P. Johansson and J. Olsen, J. Chem. Theory Comput. 4, 1460 (2008).

[63] Supporting information (SI), can be found at http://dx.doi.org/10.1021/ct1006295.

[64] D. Rappoport and F. Furche, J. Chem. Phys. 133, 134105 (2010).

[65] O. Lehtonen, D. Sundholm, R. Send, and M. P. Johansson, J. Chem. Phys. 131, 024301 (2009).

[66] R. Send, D. Sundholm, M. P. Johansson, and F. Pawłowski, J. Chem. Theory Comput. 5, 2401 (2009). 
[67] T. J. Lee and P. R. Taylor, Int. J. Quantum. Chem. Symp. S23, 199 (1989).

[68] D. Jacquemin, E. A. Perpete, I. Ciofini, C. Adamo, R. Valero, Y. Zhao, and D. G. Truhlar, J. Chem. Theory Comput. 6, 2071 (2010).

[69] D. Jacquemin, E. A. Perpete, G. Scalmani, M. J. Frisch, R. Kobayashi, and C. Adamo, J. Chem. Phys. 126, 144105 (2007).

[70] H. Eshuis, J. Yarkony, and F. Furche, J. Chem. Phys. 132, 234114 (2010).

[71] R. J. Cave, F. Zhang, N. T. Maitra, and K. Burke, Chem. Phys. Lett. 389, 39 (2004).

[72] H. E. Nikolajewski, S. Dähne, D. Leupold, and B. Hirsch, Tetrahedron 24, 6685 (1968).

[73] B. Grimm, S. Dähne, and G. Bach, Journal f. prakt. Chemie 317, 161 (1975).

[74] O. Gürtler and S. Dähne, Z. phys. Chemie, Leipzig 255, 501 (1974).

[75] S. S. Malhotra and M. C. Whiting, J. Chem. Soc. 3812 (1960).

[76] W. König and W. Regner, Ber. dtsch. chem. Ges. 63, 2823 (1930).

[77] G. Herzberg, Molecular Spectra and Molecular Structure, Vol. III (Van Nostrand and Reinhold, New York, 1966).

[78] M. Dierksen and S. Grimme, J. Chem. Phys. 120, 3544 (2004). 


\section{List of publications}

- O. Valsson and C. Filippi, "Photoisomerization of Model Retinal Chromophores: Insight from Quantum Monte Carlo and Multiconfigurational Perturbation Theory",

J. Chem. Theory Comput. 2010, 6, 1275-1292 (Chapter 3)

- R. Send, O. Valsson, and C. Filippi, "Electronic Excitations of Simple Cyanine Dyes: Reconciling Density Functional and Wave Function Methods",

J. Chem. Theory Comput. 2011, 7, 444-455 (Chapter 7)

- O. Valsson and C. Filippi,

"Gas-Phase Retinal Spectroscopy: Temperature Effects Are But a Mirage", J. Phys. Chem. Lett. 2012, 3, 908-912 (Chapter 5)

- O. Valsson, C. Angeli, and C. Filippi, "Excitation Energies of Retinal Chromophores: Critical Role of the Structural Model", Phys. Chem. Chem. Phys. 2012, 14, 11015-11020 (Chapter 4)

- C. Daday, O. Valsson, C. König, C. Filippi, and J. Neugebauer, "State-Specific Embedding Potentials for Excitation-Energy Calculations", Submitted 2012

- O. Valsson, P. Campomanes, I. Tavernelli, U. Röthlisberger, and C. Filippi, "Native Description of Visual Absorption", In preparation (Chapter 6) 



\section{Curriculum Vitae}

\section{Omar Valsson}

- Icelandic nationality.

- Born on the 8th of March 1983 in Reykjavik, Iceland.

\section{Education}

- Ph.D. in Physics, University of Twente, The Netherlands, September 2012 Supervisor: Prof. Claudia Filippi

- M.Sc. in Physics, University of Iceland, Iceland, June 2008 Supervisor: Prof. Vidar Gudmundsson Thesis title: Geometrical effects in transport through quantum wires with sidecoupled quantum dots

- B.Sc. in Physics, University of Iceland, Iceland, June 2006

- High School degree, Menntaskolinn vid Sund, Iceland, June 2003

\section{Talks given during the Ph.D. studies}

- Native Description of Visual Absorption: A Challenge for First Principles Calculations?

Meeting of the NWO-CW Study group on Spectroscopy and Theory June 15, 2012, Utrecht, The Netherlands

- Native Description of Visual Absorption: A Challenge for First Principles Calculations?

Seminar series of Michele Parrinello's research group May 21, 2012, ETH Zurich, Lugano, Switzerland

- Native Description of Visual Absorption: A Challenge for First Principles Calculations?

Seminar series of the Sector of Statistical and Biological Physics

May 18, 2012, SISSA, Trieste, Italy 
- Simulation of Photoactive Bio-Systems: Retinal Chromophore and Rhodopsin Seminar series of the Physics Department

December 16, 2010, University of Iceland, Iceland

- Quantum Monte Carlo Calculations of Photoexcitations in Bio-Molecular Systems

Psi-k conference 2010

September 12-16, 2010, Berlin, Germany

- Photoisomerization of Retinal Chromophore: Insight from Highly-Correlated Calculations

Seminar, Laboratory of Computational Chemistry and Biochemistry

March 30, 2010, EPF Lausanne, Switzerland

- Photoisomerization of Retinal Chromophore: Insight from Highly-Correlated Calculations

NWO-CW scientific meeting on Chemistry related to Physics and Material Sciences

February 15-16, 2010, Veldhoven, The Netherlands

\section{Poster presentations during the Ph.D. studies}

- Color Vision from First Principles CHAINS2011 (Chemistry As INnovating Science)

November 28-30, 2011, Maarssen, The Netherlands

- Predicting Color Tuning in Opsin Proteins: A Difficult Case for First-Principle Methods?

CPMD Meeting 2011: Extending the limits of Ab initio Molecular Dynamics Simulations for Chemistry, Materials Science and Biophysics

September 5-9, 2011, Barcelona, Spain,

- Color Tuning of Retinal Chromophore by Rhodopsin Protein Environment NWO-CW scientific meeting on Chemistry related to Physics and Material Sciences

March 14-15, 2011, Veldhoven, The Netherlands

- Photoisomerization of Model Retinal Chromophores: Insight from Quantum Monte Carlo and multiconfigurational perturbation theory

4th Time-Dependent Density Functional Theory School and Workshop January 2-15, 2010, Benasque, Spain

- Quantum Monte Carlo and CASPT2 Study of the Photoisomerization of Retinal Chromophore Models

Summer School on Simulation Approaches to Problems in Molecular and Cellular Biology

August 31-September 5, 2009, San Sebastian, Spain 


\section{Tutorials, Workshops, and Visits during the Ph.D. studies}

- The 2nd CP2K Tutorial: Enabling the Power of Imagination in MD Simulations

February 7-11, 2011, Zurich, Switzerland

- Collaborative visit of two months in the group of Prof. Ursula Roethlisberger at EPFL

March and April 2010, EPFL, Lausanne, Switzerland

- The 4th Time-Dependent Density-Functional Theory School and Workshop January 2-15, 2010, Benasque, Spain

- Summer School on Simulation Approaches to Problems in Molecular and Cellular Biology

August 31 - September 5, 2009, San Sebastian, Spain 



\section{Acknowledgements}

First and foremost, I would like to give sincere gratitude to my advisor, Claudia Filippi. Her guidance and support has been invaluable throughout my Ph.D. studies. Furthermore, I greatly appreciate her advice concerning my future scientific career.

Thanks go to my former advisor at the University of Iceland, Vidar Gudmundsson, who four years ago encouraged my to go for this Ph.D. degree, it is a decision than I have never regretted taking.

During my Ph.D. studies, I had the enjoyable experience of collaborating on different projects with numerous people outside the University of Twente. There, I would like to thank, among others, Robert Send and Celestino Angeli.

For the collaboration on subsystem DFT embedding, I express great thanks to Johannes Neugebauer and Carolin König at the Technical University Braunschweig, and, furthermore, my fellow group member Csaba Daday.

In relation to the Rhodopsin calculations, I had a long-standing collaboration with the group of Ursula Röthlisberger at the EPFL in Lausanne. There, I would like to recognize the people involved: Pablo Campomanes, Marilisa Neri, Ivano Tavernelli, and Ursula Röthlisberger. Special thanks go to Ursula for allowing me to visit her group for two months at the start of the collaboration.

Then, I would like to acknowledge all of the Ph.D. students, post-docs, and visitors, who overlapped with my stay at the University of Twente: Paul, Anton, Menno, Diana, Rien, Danny, Thijs, Süleyman, Deniz, Zhicheng, Tom, Igor, Li, Barry, Gabriel, Ioana, Francesco, and Csaba.

Finally, I am immensely grateful for the everlasting support of my family, they may have been far away in distance but never in mind. 


